\title{
Molecular Cloud Cores with a High Deuterium Fraction : Nobeyama Single-pointing Survey
}

\section{Kim, Gwanjeong}

2020-08

Kim , G , Tatematsu , K , Liu , T , Yi , H-W , He , J , Hirano , N , Liu , S-Y , Choi , M , Sanhueza , P , Toth , L V , Evans , N J , Feng , S , Juvela , M , Kim , K-T , Vastel , C , Lee , J-E , Lu'o'ng, Q N , Kang , M , Ristorcelli , I, Feher , O , Wu , Y, Ohashi , S , Wang , K , Kandori , R , Hirota , T , Sakai , T , Lu , X, Thompson , M A , Fuller , G A , Li , D , Shinnaga , H \& Kim , J 2020 , ' Molecular Cloud Cores with a High Deuterium Fraction : Nobeyama

Single-pointing Survey ' , Astrophysical Journal Supplement Series , vol. 249 , no. 2 , 33 . https://doi.org/10.3847/153

http://hdl.handle.net/10138/320416

https://doi.org/10.3847/1538-4365/aba746

other

acceptedVersion

Downloaded from Helda, University of Helsinki institutional repository.

This is an electronic reprint of the original article.

This reprint may differ from the original in pagination and typographic detail.

Please cite the original version. 
DrAFt VERSION JULY 27, 2020

Typeset using $\mathrm{LAT}_{\mathrm{E}} \mathrm{X}$ twocolumn style in AASTeX62

Molecular Cloud Cores with High Deuterium Fraction: Nobeyama Single-Pointing Survey

Gwanjeong Kim, ${ }^{1}$ Ken'ichi Tatematsu, ${ }^{1,2}$ Tie Liu, ${ }^{3}$ et al.

\author{
${ }^{1}$ Nobeyama Radio Observatory, National Astronomical Observatory of Japan, National Institutes of Natural Sciences, Nobeyama, \\ Minamimaki, Minamisaku, Nagano 384-1305, Japan \\ ${ }^{2}$ Department of Astronomical Science, SOKENDAI (The Graduate University for Advanced Studies), 2-21-1 Osawa, Mitaka, Tokyo \\ 181-8588, Japan \\ ${ }^{3}$ Shanghai Astronomical Observatory, Chinese Academy of Sciences, 80 Nandan Road, Shanghai 200030, Peoples Republic of China
}

\begin{abstract}
We present the results of a single-pointing survey of 207 dense cores embedded in Planck Galactic Cold Clumps distributed in five different environments ( $\lambda$ Orionis, Orion A, B, Galactic plane, and high latitudes) to identify dense cores on the verge of star formation for the study of the initial conditions of star formation. We observed these cores in eight molecular lines at 76-94 GHz using the Nobeyama 45-m telescope. We find that early-type molecules (e.g., CCS) have low detection rates and that late-type molecules (e.g., $\mathrm{N}_{2} \mathrm{H}^{+}, c_{-} \mathrm{C}_{3} \mathrm{H}_{2}$ ) and deuterated molecules (e.g., $\mathrm{N}_{2} \mathrm{D}^{+}$, DNC) have high detection rates, suggesting that most of the cores are chemically evolved. The deuterium fraction $(\mathrm{D} / \mathrm{H})$ is found to decrease with increasing distance, indicating that it suffers from differential beam dilution between the $\mathbf{D} / \mathbf{H}$ pair of lines for distant cores $(>\mathbf{1} \mathrm{kpc})$. For $\lambda$ Orionis, Orion $\mathrm{A}$, and $\mathrm{B}$ located at similar distances, $\mathrm{D} / \mathrm{H}$ is not significantly different, suggesting that there is no systematic difference in the observed chemical properties among these three regions. We identify at least eight high $\mathrm{D} / \mathrm{H}$ cores in the Orion region and two at high latitudes, which are most likely to be close to the onset of star formation. There is no clear evidence of the evolutionary change in turbulence during the starless phase, suggesting that the dissipation of turbulence is not a major mechanism for the beginning of star formation as judged from observations with a beam size of $0.04 \mathrm{pc}$.
\end{abstract}

Keywords: ISM: clouds — ISM: molecules — ISM: chemistry — stars: formation

\section{INTRODUCTION}

In the process of star formation, the initial condition is believed to determine what kinds of stars will be born and how they will form (e.g., Bergin \& Tafalla 2007; McKee \& Ostriker 2007). However, the details of initial conditions have not been thoroughly characterized.

Stars are formed through gravitational collapse in dense molecular cores (e.g., Bergin \& Tafalla 2007; McKee \& Ostriker 2007), and many observations have reported that the core mass function is similar in shape to the initial mass function (e.g., André et al. 2010; Könyves et al. 2010). However, this is not the case in high-mass regimes, where deviations from the Salpeter IMF have been recently found (e.g., Zhang et al. 2015; Motte et al. 2018; Sanhueza et al. 2019). These studies suggest that studying dense cores in different mass ranges may provide valuable information on the initial conditions of star formation. Furthermore, we need to take care of the evolution in the starless phase because

Corresponding author: Gwanjeong Kim

gj.kim@nao.ac.jp the internal structure of dense cores is altered physically and chemically during the core evolution (Aikawa et al. 2008). Therefore, the identification of the dense core just prior to the beginning of gravitational collapse, which we call 'prestellar core' here, is essential in understanding the genuine initial conditions of star formation (e.g., Ohashi et al. 2018).

In low-mass star-forming regions, prestellar cores are not only compact $(\lesssim 0.1 \mathrm{pc})$, cold $(\lesssim 10 \mathrm{~K})$, and dense $\left(>5 \times 10^{4} \mathrm{~cm}^{-3}\right)$ (e.g., Bergin \& Tafalla 2007; McKee \& Ostriker 2007), but also gravitationally bound, thermally supported, and centrally concentrated (e.g., Ward-Thompson et al. 1994; Caselli 2011). For highmass star formation, prestellar cores are as massive as at least $30 M_{\odot}$, assuming a star formation efficiency of 30\% (Wang et al. 2014; Sanhueza et al. 2019).

The chemical characteristic of starless cores close to the onset of star formation is high deuterium fractionation. The deuterium fraction $(\mathrm{D} / \mathrm{H}$, hereafter) is defined as a column-density ratio of a deuterated molecule to its hydrogenated counterpart, and has commonly been used to study the chemical properties of dense cores in lowmass star-forming regions as well as in high-mass starforming regions (e.g., Hirota et al. 2001; Crapsi et al. 
2005; Hirota \& Yamamoto 2006; Bergin \& Tafalla 2007; Chen et al. 2010, 2011; Fontani et al. 2011; Sakai et al. 2012, 2015, 2018; Feng et al. 2019).

The chemical evolution of a dense core in terms of $\mathrm{D} / \mathrm{H}$ can be described as follows. As a dense core evolves toward gravitational collapse, it develops a steep radial density profile that increases toward the core center and a radial temperature profile that decreases toward the core center (e.g., Crapsi et al. 2007; Aikawa et al. 2008). At the core center $(\lesssim 5000$ AU) with low temperature $\left(T_{\text {dust }} \lesssim 25 \mathrm{~K}\right.$ and often closer to $\left.10 \mathrm{~K}\right)$ and high density $\left(n_{\mathrm{H}_{2}} \geq 10^{4} \mathrm{~cm}^{-3}\right)$, CO molecules (a destroyer of deuterated molecules) become frozen on the surface of the dust grain and the enrichment of deuterated molecules is activated with exothermic ion-molecule reactions from HD molecules (e.g., Millar et al. 1989; Phillips \& Vastel 2003; Vastel et al. 2004; Aikawa et al. 2005; Crapsi et al. 2005). When a protostar is born at the core center, it heats up the surroundings (e.g., Caselli et al. 2002b). At the warm $\left(T_{\text {dust }} \gtrsim 25 \mathrm{~K}\right)$ core center, CO molecules are desorbed from the grain surface and then destroy deuterated molecules (Roberts \& Millar 2000; Lee et al. 2004). Therefore, $\mathrm{D} / \mathrm{H}$ varies with temperature. If the core is cold enough $\left(T_{\text {dust }}<25 \mathrm{~K}\right), \mathrm{D} / \mathrm{H}$ increases in the starless phase and reaches its maximum at the onset of star formation, and then decreases by an order of magnitude after star formation (e.g., Hirota et al. 2003; Crapsi et al. 2005; Emprechtinger et al. 2009; Friesen et al. 2010; Sakai et al. 2012; Fontani et al. 2014).

$N(\mathrm{DNC}) / N(\mathrm{HNC})$ and $N\left(\mathrm{~N}_{2} \mathrm{D}^{+}\right) / N\left(\mathrm{~N}_{2} \mathrm{H}^{+}\right)$can be used as $\mathrm{D} / \mathrm{H}$ tracers. $\mathrm{N}_{2} \mathrm{D}^{+}$is known to be the least depleted molecule (Pagani et al. 2007) and DNC is known to trace cold molecular gas (Gerner et al. 2015). The destruction timescale of the DNC molecule is known to be much longer than that of the $\mathrm{N}_{2} \mathrm{D}^{+}$molecule (e.g., Sakai et al. 2012; Fontani et al. 2014). For example, after stellar birth, $N\left(\mathrm{~N}_{2} \mathrm{D}^{+}\right) / N\left(\mathrm{~N}_{2} \mathrm{H}^{+}\right)$decreases within 100 years, whereas $N(\mathrm{DNC}) / N(\mathrm{HNC})$ drops at a timescale of $10^{4}$ years. Fontani et al. (2014) showed that the deuterium fractionation of $\mathrm{N}_{2} \mathrm{H}^{+}$is more suitable than that of HNC to identify cores on the verge of star formation in high-mass star-forming regions.

For the chemical evolution of the dense core, it is also noticed that the abundance of gas-phase molecules decreases at different timescales because of adsorption and/or chemical reactions in the central dense region of the core (Aikawa et al. 2001, 2003; Lee et al. 2003). For example, carbon-chain molecules (e.g., $\mathrm{CCS}, \mathrm{HC}_{3} \mathrm{~N}$ ) are abundant in starless cores, whereas $c-\mathrm{C}_{3} \mathrm{H}_{2}$ and nitrogen-bearing molecules (e.g., $\mathrm{N}_{2} \mathrm{H}^{+}, \mathrm{NH}_{3}$ ) are abundant in protostellar cores (e.g., Suzuki et al. 1992; Benson et al. 1998; Ohashi et al. 2014, 2016). That is, CCS molecules are known as 'early-type species' and $\mathrm{c}-\mathrm{C}_{3} \mathrm{H}_{2}, \mathrm{~N}_{2} \mathrm{H}^{+}$, and $\mathrm{NH}_{3}$ molecules are known as 'late-type species'. This means that chemically evolved cores have lower abundances of $\mathrm{C}$-chain molecules and higher abundances of $\mathrm{c}_{-} \mathrm{C}_{3} \mathrm{H}_{2}$ and N-bearing molecules.
$N\left(\mathrm{~N}_{2} \mathrm{H}^{+}\right) / N(\mathrm{CCS})$ and $N\left(\mathrm{~N}_{2} \mathrm{H}^{+}\right) / N\left(\mathrm{HC}_{3} \mathrm{~N}\right)$ can be valid chemical evolution tracers at a spatial size scale of $\sim 0.1$ pc for cold $\left(T_{\text {dust }} \lesssim 25 \mathrm{~K}\right)$ cores. These ratios are invalid as evolution tracers for warm $(>25 \mathrm{~K})$ cores, because the $\mathrm{N}_{2} \mathrm{H}^{+}$molecule is destroyed by $\mathrm{CO}$ desorption (e.g., Aikawa et al. 2001, 2003; Lee et al. 2003; Tatematsu et al. 2014).

Measurements of the column-density ratios of deuterated/hydrogenated molecules and N-bearing/C-chain molecules provide a useful tool to characterize the evolutionary status of the dense core in which a star will form (e.g., Hirota \& Yamamoto 2006). In this paper, $\mathrm{HC}_{3} \mathrm{~N}$ is regarded as a $\mathrm{C}$-chain molecule rather than an N-bearing molecule. Taking chemical evolution into account, Tatematsu et al. (2017) introduced the Chemical Evolution Factor (CEF, hereafter) as a comprehensive diagnostic tool for the degree of evolution of dense cores. The CEF is defined so that, when a dense core evolves, the CEF monotonically increases from $\sim-100$ for a starless core to $\sim 100$ for a protostellar core through zero corresponding to the onset of star formation (See Section 4.1). The first attempt was made toward low-mass starless cores in nearby cold clouds in the literature (Tatematsu et al. 2017), but the CEF should be established for other environments (e.g., distant high-mass starless cores) by increasing sample numbers.

Efforts toward the identification of dense cores over all sky have extended from optical to infrared wavelengths with instrumental development (e.g., dark cloud in DSS, infrared dark cloud in MSX and Spitzer, Lee \& Myers 1999; Simon et al. 2006; Peretto \& Fuller 2009; Kim et al. 2010; Dobashi 2011). Most recently, the Planck all-sky survey has been made and provides a catalog of Planck Galactic Cold Clumps (PGCC, hereafter) (Planck Collaboration et al. 2011, 2016), which includes 13,188 sources of $\sim 5$-10 arcmin-sized structures "clumps" with temperatures of $10-20 \mathrm{~K}$ in various environments. An unbiased selection of PGCCs was mapped with Herschel in far-IR band (e.g., Juvela et al. 2010, 2012, 2018). A series of follow-up observations have been conducted with ground-based radio telescopes such as PMO, CSO, SMT, APEX, NANTEN2, IRAM, Mopra, Effelsberg, SMA, JCMT, and TRAO, and are summarized by Liu et al. (2015, 2018). Specifically, the JCMT large program "SCUBA-2 Continuum Observations of Pre-protostellar Evolution (SCOPE)" identified 20-60 arcsec-sized structures or "cores" within PGCCs (Liu et al. 2018). These PGCC cores are distributed in low/high-mass star-forming regions from the Galactic plane to high latitudes (Eden et al. 2019). The initial conditions of star formation in these various environments are still poorly understood. For example, the initial conditions of high-mass star formation are poorly known and debatable because of the small numbers of massive prestellar cores in high-mass star-forming regions so far identified (e.g., Wang et al. 2014; Contreras et al. 2018; Sanhueza et al. 2017, 2019) because of their 


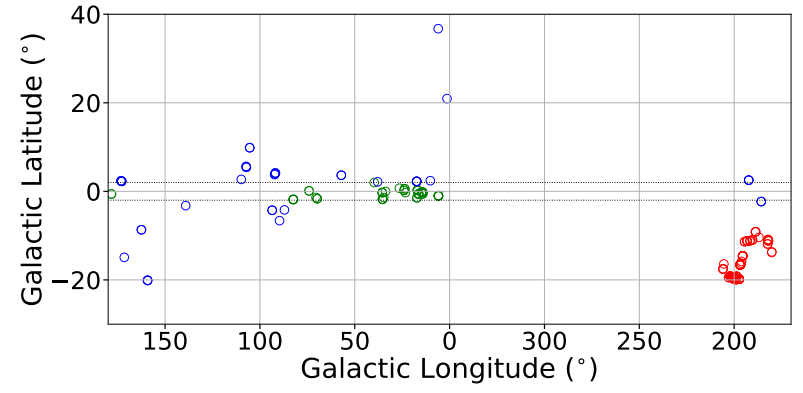

Figure 1. Galactic distribution of 207 SCUBA-2 cores. They consist of GMC cores in the Orion region (red), cores in the Galactic plane (green), and cores at high latitudes (blue). The dotted line represents Galactic latitudes of $\pm 2^{\circ}$.

large distances and short evolutionary timescale (McKee \& Ostriker 2007; Caselli 2011). These PGCC cores can be valuable targets not only to assess evolutionary stages on the basis of the column-density ratios of $\mathrm{D} / \mathrm{H}$ and N-bearing/C-chain molecules, but also to study the initial conditions of star formation in widely different environments.

Our ultimate goal is to explore the details of the initial conditions for star formation based on the physical and chemical properties of prestellar cores in various environments. The first step is to identify reliable samples of such dense cores. Thus, we report the first stage of our research with single-pointing observations of 207 cores in PGCCs in the molecular lines of $\mathrm{N}_{2} \mathrm{D}^{+} J=1-0$, $\mathrm{N}_{2} \mathrm{H}^{+} J=1-0$, DNC $J=1-0, \mathrm{HN}^{13} \mathrm{C} J=1-0$, CCS $J_{N}=7_{6}-65, \operatorname{CCS} J_{N}=8_{7}-7_{6}, \mathrm{HC}_{3} \mathrm{~N} J=9-8$, and $\mathrm{c}-\mathrm{C}_{3} \mathrm{H}_{2} J_{K_{a} K_{c}}=2_{12}-1_{01}$ with the 45 -m single-dish telescope of the Nobeyama Radio Observatory ${ }^{1}$. Cores found in this study are in the chemically evolved stage of starless cores (late starless core, hereafter) as prestellar core candidates. In the next paper, we will investigate whether these late starless cores are actually prestellar cores or not through mapping observations.

This study is organized as follows: observations and data analysis procedures are described in Section 2, the observational results are presented in Section 3, Section 4 presents the identification of late starless cores in various environments using the $\mathrm{CEF}$ and $\mathrm{D} / \mathrm{H}$, and the study is summarized in Section 5 .

\section{OBSERVATIONS AND DATA ANALYSIS PROCEDURE}

\subsection{Sample selection}

The SCOPE survey discovered more than 3,000 PGCC cores across the Galaxy at $850 \mu \mathrm{m}$ with SCUBA-

\footnotetext{
${ }^{1}$ Nobeyama Radio Observatory is a branch of the National Astronomical Observatory of Japan, National Institutes of Natural Sciences.
}

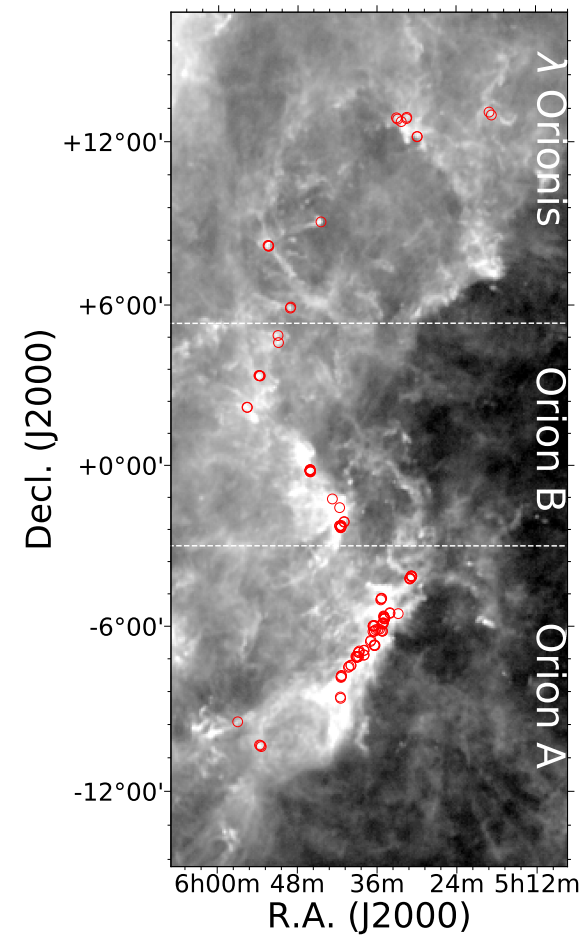

Figure 2. Spatial distribution of SCUBA-2 cores in the Orion region. The background image is the Planck dust continuum map at $850 \mu \mathrm{m}$ with a size of $15^{\circ} \times 30^{\circ}$. The symbol is the same as those used in Figure 1. The horizontal lines represent the boundaries for three sub-regions ( $\lambda$ Orionis, Orion A, and Orion B).

2 (Submillimetre Common-User Bolometer Array 2) on board JCMT (e.g., Yi et al. 2018; Eden et al. 2019). Yi et al. (2018) identified 119 cores embedded in 96 PGCCs in the Orion region from JCMT SCOPE data and JCMT archive data. Eden et al. (2019) released a compact source catalog using the JCMT SCOPE data, which contains 3,528 cores identified in 1,235 PGCCs from the Galactic plane to high latitudes. As a pilot study, we selected a total of 207 targets that are located at different Galactic positions (local Giant Molecular Clouds (GMCs), high latitude, and Galactic plane). These dense cores are among the densest cores $\left(N\left(\mathrm{H}_{2}\right)\right.$ $>1 \times 10^{22} \mathrm{~cm}^{-2}$ ) discovered in the SCOPE survey and consist of 113 cores in the Orion region, which is associated with GMCs, 52 cores in the Galactic plane $\left(|b|<2^{\circ}\right)$, and 42 cores at high latitudes $\left(|b| \geq 2^{\circ}\right)$. Figure 1 shows the Galactic distribution of 207 SCUBA-2 cores, and the information on the targets is summarized in Table 1 with the core name, coordinate, dust temperature, $\mathrm{H}_{2}$ column density, type of environment, and the related PGCC. The dust temperature is taken from the PGCC catalog and the $\mathrm{H}_{2}$ column density is derived from the $850 \mu \mathrm{m}$ peak intensity of the PGCC core (Yi et al. 2018; Eden et al. 2019) and the dust temperature. The 207 SCUBA-2 cores have dust temperatures rang- 
ing from $9.2 \mathrm{~K}$ to $22.4 \mathrm{~K}$ with the median value of 13.3 $\mathrm{K}$. They have $\mathrm{H}_{2}$ column densities of $(0.17-12) \times 10^{23}$ $\mathrm{cm}^{-2}$ with the median value of $8.2 \times 10^{22} \mathrm{~cm}^{-2}$.

We investigate environments surrounding SCUBA-2 cores using the SIMBAD database. The Orion region, including the 113 SCUBA-2 cores, is a local active starforming region where both massive stars and low-mass stars are born (e.g., Yi et al. 2018). This region consists of $\lambda$ Orionis, Orion A, and B sub-regions, which include GMCs and many star-forming regions such as HII regions (e.g., LDN 1641 S3), star clusters (e.g., Collinder 69 , Orion Nebula Cluster, NGC 2023, OMC 2), and nebulas (e.g., Orion KL, McNeil Nebula, IC 432). 15, 70, and 28 SCUBA-2 cores are located in the $\lambda$ Orionis, Orion A, and Orion B, respectively, and their distributions are shown in Figure 2. Yi et al. (2018) found that the physical properties of the SCUBA-2 cores in the three sub-regions are different. The SCUBA-2 cores correspond to part of the dark clouds (e.g., L1581, L1582, L1594, L1598, L1630, and L1641).

The 52 SCUBA-2 cores in the Galactic plane are located in the vicinity of open clusters (e.g., NGC2264, NGC6530, NGC6611) and supernova remnants (e.g., W48) and are embedded in dark clouds (e.g., L463). The 42 SCUBA-2 cores at high latitudes seem to be associated with famous star-forming regions (e.g., Auriga, California, Cepheus, Cygnus, IC5146, Ophiuchus, Perseus, and Taurus), nearby dark clouds (e.g., Banard 1, L43, L183, L769, L944, L973, L1004, L1181, L1204, L1035, L1521F, and L1525), open clusters (e.g., NGC2264), and star clusters (e.g., NGC7129).

The information on these environments is listed in Table 1 . We consider $\lambda$ Orionis, Orion A, B, Galactic plane, and high latitudes as five different environments.

\subsection{Single-pointing observations}

We carried out single-pointing observations toward the $850 \mu \mathrm{m}$ intensity peak position of 207 SCUBA2 cores with the 45-m single-dish radio telescope of the Nobeyama Radio Observatory from 2017 February to 2018 May (CG161004, LP177001, P.I.: K. Tatematsu). Either of the two-sideband SIS receivers, T70 or TZ, was used for simultaneous four-line observations in the double-polarization mode (Asayama \& Nakajima 2013; Nakajima et al. 2013). The T70 receiver was adopted for observations towards all 207 SCUBA-2 cores in the $\mathrm{c}_{-} \mathrm{C}_{3} \mathrm{H}_{2} J_{K_{a} K_{c}}=212-1_{01}$, DNC $J=1-0$, $\mathrm{HN}^{13} \mathrm{C} J=1-0$, and $\mathrm{N}_{2} \mathrm{D}^{+} J=1-0$ molecular emission lines. The TZ receiver was used for observations towards 111 SCUBA-2 cores in the Orion region in the CCS $J_{N}=7_{6}-6_{5}$ (CCS-L, hereafter), CCS $J_{N}=8_{7}-7_{6}(\mathrm{CCS}-\mathrm{H}$, hereafter $), \mathrm{HC}_{3} \mathrm{~N} J=9-8$, and $\mathrm{N}_{2} \mathrm{H}^{+} J=1-0$ lines. Observations with the TZ receiver were not conducted for two Orion cores and all cores in environments other than the Orion region due to time limitations. The rest frequencies and their references, upper energy levels, and employed receivers of the eight
Table 2. Molecular lines

\begin{tabular}{lcccc}
\hline \hline \multicolumn{1}{c}{ Molecular Line } & Freq. (GHz) & Ref. & $\mathrm{E}_{u}(\mathrm{~K})$ & Receiver \\
\hline $\mathrm{DNC} J=1-0$ & 76.305717 & 1 & 3.7 & $\mathrm{~T} 70$ \\
$\mathrm{~N}_{2} \mathrm{D}^{+} J=1-0$ & 77.109610 & 2 & 3.7 & $\mathrm{~T} 70$ \\
$\mathrm{c}^{-} \mathrm{C}_{3} \mathrm{H}_{2} J_{K_{a} K_{c}}=2_{12}-1_{01}$ & 85.338906 & 3 & 4.1 & $\mathrm{~T} 70$ \\
$\mathrm{HN}^{13} \mathrm{C}=1-0$ & 87.090859 & 4 & 4.2 & $\mathrm{~T} 70$ \\
$\mathrm{CCS} \mathrm{J}_{N}=7_{6}-6$ & 81.505208 & 5 & 15.3 & $\mathrm{TZ}$ \\
$\mathrm{HC}_{3} \mathrm{~N} J=9-8$ & 81.881461 & 6 & 19.7 & $\mathrm{TZ}$ \\
$\mathrm{N}_{2} \mathrm{H}^{+} J=1-0$ & 93.173777 & 7 & 4.5 & $\mathrm{TZ}$ \\
$\mathrm{CCS} J_{N}=8_{7}-7_{6}$ & 93.870107 & 8 & 19.9 & $\mathrm{TZ}$ \\
\hline
\end{tabular}

Note-Column 1: Name of the molecular line, Column 2: Rest frequency for the molecular line, Column 3: Reference for the employed frequency: 1. Okabayashi \& Tanimoto (1993), 2. Anderson et al. (1977), 3. Thaddeus et al. (1985), 4. Frerking et al. (1979), 5. Cummins et al. (1986), 6. Pickett et al. (1998), 7. Caselli \& Myers (1995), 8. Yamamoto et al. (1990), Column 4: Upper energy level of the molecular line, Column 5: Receiver used to observe the molecular line.

lines are listed in Table 2. At $82 \mathrm{GHz}$, the half power beam widths (HPBW) of the T70 and TZ receivers are $19^{\prime \prime} 5 \pm 0{ }^{\prime \prime} 3$ and $18^{\prime \prime} .8 \pm 00^{\prime \prime} 1$, respectively. Their main-beam efficiencies $\eta_{m b}$ are $54.7 \pm 3.5 \%$ and $55.4 \pm 3.5 \%$, respectively. The FX digital spectrometer SAM45 (Kamazaki et al. 2012) was employed with a spectral resolution of $15.26 \mathrm{kHz}$ (corresponding to $\sim 0.06 \mathrm{~km} \mathrm{~s}^{-1}$ at $82 \mathrm{GHz}$ ) for 113 SCUBA-2 cores in the Orion region and 30.52 $\mathrm{kHz}$ (corresponding to $\sim 0.1 \mathrm{~km} \mathrm{~s}^{-1}$ at $82 \mathrm{GHz}$ ) for the remaining 94 cores. The use of two different spectral resolutions was accidental. The system temperature was typically $200 \mathrm{~K}$.

Single-pointing observation for each core typically took $\sim 30$ minutes, including the telescope overhead time in the position-switching mode, to achieve 0.09 $\mathrm{K} \mathrm{rms}$ sensitivity at a resolution of $0.1 \mathrm{~km} \mathrm{~s}^{-1}$. The telescope pointing uncertainty was established as $\lesssim 5^{\prime \prime}$ through five-point measurement toward $43 \mathrm{GHz} \mathrm{SiO}$ maser sources close to targets every $\sim 1-1.5$ hours. The resulting spectrum is expressed in terms of the antenna temperature corrected for atmosphere extinction $T_{\mathrm{A}}^{*}$ obtained by the standard chopper wheel calibration. For the reduction of raw data, the baselines are subtracted and then the spectra are co-added on the software package "NEWSTAR" 2 of the Nobeyama Radio Observatory. The reduced data are transferred into a data format of the GILDAS CLASS program ${ }^{3}$ and are resampled into a $0.1 \mathrm{~km} \mathrm{~s}^{-1}$ velocity resolution.

\subsection{Gaussian or Hyperfine structure fitting to a spectrum}

Figure 3 shows the spectra of four or eight molecular emission lines for SCUBA-2 cores. $\mathrm{N}_{2} \mathrm{H}^{+}$and $\mathrm{N}_{2} \mathrm{D}^{+}$ lines show the seven components of hyperfine transi-

\footnotetext{
2 https://www.nro.nao.ac.jp/ nro45mrt/html/obs/newstar/

${ }^{3}$ https://www.iram.fr/IRAMFR/GILDAS/
} 
tions $^{4}$ (e.g., Caselli et al. 2002b), but the other lines generally exhibit a single velocity component. For a further analysis, we make a Gaussian (GA) or hyperfine structure (HFS) fitting to each spectrum using the GILDAS CLASS program. For $\mathrm{c}^{-} \mathrm{C}_{3} \mathrm{H}_{2}, \mathrm{CCS}$, and $\mathrm{HC}_{3} \mathrm{~N}$ lines having single Gaussian shapes, we apply the GA fitting to them to measure the peak temperature $\left(T_{\text {peak }}\right)$, systemic velocity $\left(V_{\mathrm{LSR}}\right)$, and linewidth $(\Delta v)$ in Full Width at Half Maximum (FWHM). DNC and $\mathrm{HN}^{13} \mathrm{C}$ lines look like single Gaussian shapes, but they are known to have four and six components of hyperfine transitions, respectively (van der Tak et al. 2009). Because our main purpose is to derive the column density, for simplicity we ignore the hyperfine splitting for $\mathrm{DNC}$ and $\mathrm{HN}^{13} \mathrm{C}$, and apply simple Gaussian fitting. The derived linewidths of both lines are overestimated for this reason. For $\mathrm{N}_{2} \mathrm{D}^{+}$ and $\mathrm{N}_{2} \mathrm{H}^{+}$lines, where the seven components of hyperfine transitions are clearly visible, we apply the HFS fitting to them to obtain the excitation temperature $\left(T_{\mathrm{ex}}\right)$, $V_{\mathrm{LSR}}, \Delta v$, optical depth $(\tau)$, and $T_{\text {ant }} \tau$. To obtain $T_{\text {peak }}$ and $\Delta v$ of the brightest component $\left(J F_{1} F=123\right.$ 012 ) of the $\mathrm{N}_{2} \mathrm{H}^{+}$and $\mathrm{N}_{2} \mathrm{D}^{+}$lines, we apply the GA fitting to the brightest component of the lines. We measure the rms $(\sigma)$ noise level from the baseline subtraction procedure for the spectrum. The fitting results are listed in Tables 3 and 4 . We regard the peak temperature higher than $3 \sigma$ as detection for further analysis. Otherwise, we give $3 \sigma$ as an upper limit and consider it as non-detection.

\subsection{Distance of 207 SCUBA-2 cores}

Accurate distances of cores are often unavailable because of the lack of references. When an accurate distance to the parent cloud is known in the literature, we adopt the value. Otherwise, we employ distance from the parallax-based distance estimator of the Bar and Spiral Structure Legacy Survey (Reid et al. 2016) on the basis of the systemic velocity (Section 2.3) and the sky position of the core. With other lines, the $\mathrm{c}^{-} \mathrm{C}_{3} \mathrm{H}_{2}$ line is basically used for the systemic velocity because the line has the highest detection rate (Section 3.1). Distance and its reference are summarized in Table 1.

The median values of the employed distances are found to be $380 \mathrm{pc}$ for cores in the $\lambda$ Orionis, $430_{-40}^{+0}$ pc for cores in the Orion A, $390_{-0}^{+30}$ pc for cores in the Orion B, $2.3_{-1.3}^{+7.0} \mathrm{kpc}$ for cores in the Galactic plane, and $0.8_{-0.7}^{+1.8} \mathrm{kpc}$ for cores at high latitudes. It is noted that most of the cores in the Galactic plane are distributed farther away than cores at other environments. Orion cores are located at similar distances, but cores at other environments are widely scattered in distance.

\footnotetext{
${ }^{4}$ https://spec.jpl.nasa.gov/
}

\subsection{Classification of SCUBA-2 cores into starless and protostellar cores}

Each of the 207 SCUBA-2 cores may be either before or after stellar birth, so they need to be classified into two groups: starless cores and protostellar cores. Reliable categorization can be difficult, depending on the availability of protostellar data and references. Therefore, we simply categorize them by visually investigating the existence of a young stellar object (YSO) within the criterion radius centered at the peak position of each core. For example, if no YSO is known within the criterion radius, the core is regarded as a starless core (candidate). If a YSO is located within the criterion radius, we consider the core as a protostellar core (candidate). For simplicity, we refer to starless cores and their candidates as starless cores, and protostellar cores and their candidates as protostellar cores. The criterion radius is adopted from Yi et al. (2018) and Eden et al. (2019). The information of the YSOs is taken from the SIMBAD database including the past literature and protostar catalogs based on large programs such as Spitzer, WISE, Herschel, and GAIA missions (Megeath et al. 2012; Povich et al. 2013; Dunham et al. 2015; Marton et al. 2016, 2019).

From the visual inspection, the samples of our SCUBA-2 cores are classified into 58 starless cores and 149 protostellar cores. The 58 starless cores consist of 5 cores in the $\lambda$ Orionis, 24 in the Orion A, 10 in the Orion B, 13 in the Galactic plane, and 6 at high latitudes. Out of 149 protostellar cores, 10, 46, 18, 39, and 36 cores are located in the same five categories of environments, respectively. The classification and the associated YSOs are summarized in Table 1, but may be changed if the YSO information is updated. Considering this uncertainty, among the total 207 SCUBA-2 cores, the upper limit of the fraction of starless cores is found to be $\sim 28 \%$.

\subsection{Estimation for the column density and column-density ratio of molecules}

We derive the column densities of the six observed molecules, $\mathrm{N}_{2} \mathrm{D}^{+}, \mathrm{N}_{2} \mathrm{H}^{+}, \mathrm{DNC}, \mathrm{HN}^{13} \mathrm{C}, \mathrm{CCS}$, and $\mathrm{HC}_{3} \mathrm{~N}$ (Tables 3 and 4). Among the two transitions of CCS, we use the lower-frequency transition because it has a higher detection rate (Section 3.1). The column densities of the six molecules can be derived with the assumption that the molecular lines are under local thermodynamic equilibrium (LTE) as described in Suzuki et al. (1992), Sanhueza et al. (2012), and Mangum \& Shirley (2015). For $\mathrm{N}_{2} \mathrm{D}^{+}$and $\mathrm{N}_{2} \mathrm{H}^{+}$lines, the column density is estimated through the HFS fitting as follows. (1) When the HFS fitting is successful to some extent, the column density is estimated from $T_{\text {ant }} \tau=\left(T_{\text {ex }}-T_{\mathrm{bg}}\right) \tau$, as a main factor together with $T_{\mathrm{ex}}$ as a weaker contribution. Here, $\tau$ and $T_{\mathrm{bg}}$ are the total line optical depth of all the hyperfine components and the temperature 
of the cosmic microwave background, respectively. (1-1) If the HFS fitting is fully successful or the $T_{\text {ant }} \tau$ error is $\leq \mathbf{5 0 \%}$ and excitation temperature is in a range of $4 \mathrm{~K} \leq T_{\mathrm{ex}} \leq 25 \mathrm{~K}$, the column density is derived from $T_{\mathrm{ant}} \tau$ and $T_{\mathrm{ex}}$ from the HFS fitting. (1-1-1) In a case of the optically thin limit shown as ' $\tau=0.1$ ', $\tau$ is too small to constrain. The relative uncertainty in $T_{\text {ant }} \tau$ is small, although that in $\tau$ is large. (1-2) If the HFS fitting is partially successful or the error of $T_{\text {ant }} \tau$ is $\leq \mathbf{5 0 \%}$ but $T_{\text {ex }}$ is out of the above range, $T_{\text {ex }}$ is estimated from dust temperature. We assume that gas kinetic temperature $\left(T_{\mathrm{k}}\right)$ is equal to dust temperature, and also assume $T_{\mathrm{ex}}=0.5 T_{\mathrm{k}}$ following Tatematsu et al. (2017), who studied nine Planck Galactic clumps in the $\mathrm{NH}_{3}$ lines. (2) When the HFS fitting is not successful or the $T_{\text {ant }} \tau$ error is $>50 \%$, the column density is derived through single Gaussian fitting to the brightest hyperfine component of the $\mathbf{N}_{2} \mathrm{D}^{+}$and $\mathbf{N}_{2} \mathbf{H}^{+}$lines. The excitation temperature is, again, estimated from dust temperature. For $\mathrm{DNC}, \mathrm{HN}^{13} \mathrm{C}, \mathrm{CCS}$, and $\mathbf{H C}_{3} \mathbf{N}$ lines, the column density is calculated from the peak temperature and linewidth through the Gaussian fitting, and excitation temperature estimated from dust temperature. We estimate the uncertainty of the column density through propagation from the $1 \sigma$ fitting error and the rms noise level of the spectrum. Some cores (e.g., G205.46-14.56North1) show excitation anomalies that the ratios of the hyperfine are not fit well with a single excitation temperature (See Figure 4). Note that the Rayleigh-Jeans approximation is assumed in the HFS fitting. Such excitation anomalies are reported by Caselli et al. (1995b). We take into account excitation anomalies only as the fitting error in $T_{\text {ex }}$. When we employ excitation temperature from dust temperature, we assume that the error in excitation temperature is $50 \%$, following the studies of Tatematsu et al. (2008, 2017). However, even in this case, when $T_{\mathrm{ex}}$ is too low, we can provide only lower limits on the column density. For instance, if $T_{\mathrm{ex}}$ is lower than 6.5 $\mathrm{K}$, the line optical depth becomes moderate or large $(\tau>0.7)$ at the lower end of the $50 \%$ error range for $T_{\mathrm{ex}}$ and we cannot obtain upper limits on the column density reliably. In this case, we show lower limits on the column density obtained at the upper end of the $50 \%$ error range for $T_{\mathrm{ex}}$. For undetected lines, we derive upper limits on the column density by using the $3 \sigma$ level at a $0.5 \mathrm{~km} \mathrm{~s}^{-1}$ resolution. The column densities of six molecules are listed in Table 5.

For the column-density ratios of $\mathrm{D} / \mathrm{H}$ and $\mathrm{N}$ bearing/C-chain molecules, we calculate $N\left(\mathrm{~N}_{2} \mathrm{D}^{+}\right) /$ $N\left(\mathrm{~N}_{2} \mathrm{H}^{+}\right), \quad N(\mathrm{DNC}) / N\left(\mathrm{HN}^{13} \mathrm{C}\right), N\left(\mathrm{~N}_{2} \mathrm{H}^{+}\right) / N(\mathrm{CCS})$,

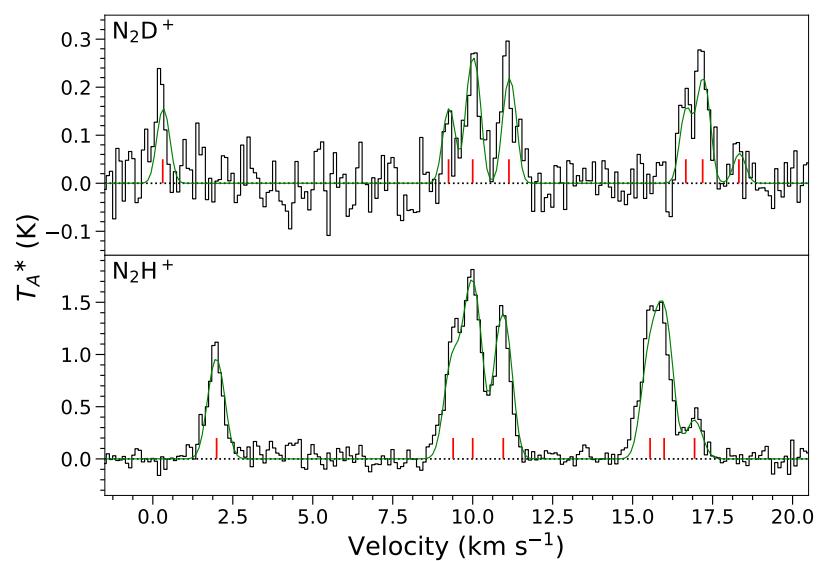

Figure 4. Examples of excitation anomalies seen in $\mathbf{N}_{2} \mathbf{D}^{+}$and $\mathbf{N}_{2} \mathbf{H}^{+}$lines. The black and green lines represent the spectrum of G205.46-14.56North1 and the result of hyperfine structure fitting, respectively. The red vertical lines represent the velocity offsets of the seven hyperfine components.

and $N\left(\mathrm{~N}_{2} \mathrm{H}^{+}\right) / N\left(\mathrm{HC}_{3} \mathrm{~N}\right)$ under the assumption that these line emissions are emitted from the same region. If one of the pairing column densities is not estimated, the lower limit or the upper limit is shown. The uncertainty of the column-density ratio is given by propagating from the error of column density in the ratio. Table 5 lists the column-density ratios.

\subsection{Mach number estimate}

We calculate the Mach number $M=\sigma_{\mathrm{NT}} / c_{\mathrm{s}}$ to measure the contribution of turbulence in the stability of the dense core. This parameter is used to judge whether a dense core is subsonic $(M \leq 1)$, transonic $(1<M \leq$ $2)$, or supersonic $(M>2)$. The non-thermal velocity dispersion $\left(\sigma_{\mathrm{NT}}\right)$ is derived in the following form (e.g., Myers 1983; Fuller \& Myers 1992; Caselli et al. 2002a):

$$
\sigma_{\mathrm{NT}}=\sqrt{\frac{\Delta v^{2}}{8 \ln 2}-\frac{k_{\mathrm{B}} T_{\mathrm{k}}}{m}},
$$

where $k_{\mathrm{B}}$ is the Boltzmann constant and $T_{\mathrm{k}}$ is the kinetic temperature. $\Delta v$ and $m$ are the linewidth (FWHM) and mass of the observed molecule, respectively. The sound speed $\left(c_{\mathrm{s}}\right)$ is estimated by $\sqrt{k_{\mathrm{B}} T_{\mathrm{k}} / m}$ for the $\mathrm{H}_{2}$ molecule.

We assume that $T_{\mathrm{k}}$ is equal to dust temperature. For $\Delta v$ and $m$, we adopt the $\mathrm{N}_{2} \mathrm{H}^{+}$molecule because it traces the dense gas. The uncertainty of the Mach number is derived by propagating from the error of the fitting, the error of dust temperature, and the rms $(\sigma)$ noise level of the spectrum. The Mach number is listed in Table 5.

\section{RESULTS}

\subsection{Detection rate of molecular emission lines}


Table 6. Detection rates of molecular lines in SCUBA-2 cores in different environments

\begin{tabular}{|c|c|c|c|c|c|c|c|c|c|}
\hline \multirow[t]{2}{*}{ Environment } & \multirow[t]{2}{*}{ Source } & $\mathrm{c}-\mathrm{C}_{3} \mathrm{H}_{2}$ & $\mathrm{DNC}$ & $\mathrm{HN}^{13} \mathrm{C}$ & $\mathrm{N}_{2} \mathrm{D}^{+}$ & CCS-L & $\mathrm{CCS}-\mathrm{H}$ & $\mathrm{HC}_{3} \mathrm{~N}$ & $\mathrm{~N}_{2} \mathrm{H}^{+}$ \\
\hline & & \multicolumn{8}{|c|}{$\%$} \\
\hline \multirow{3}{*}{ All region } & Both (207) & 91 & 78 & 74 & 49 & $\cdots$ & $\cdots$ & $\ldots$ & $\cdots$ \\
\hline & Starless core $(58)$ & 81 & 76 & 60 & 47 & $\cdots$ & $\cdots$ & $\cdots$ & $\cdots$ \\
\hline & Protostellar core (149) & 95 & 79 & 79 & 50 & $\cdots$ & $\ldots$ & $\ldots$ & $\ldots$ \\
\hline \multirow{3}{*}{$\lambda$ Orionis } & Both (15) & 80 & 67 & 60 & 40 & 13 & 13 & 47 & 80 \\
\hline & Starless core $(5)$ & 40 & 40 & 20 & 20 & 0 & 20 & 20 & 60 \\
\hline & Protostellar core (10) & 100 & 80 & 80 & 50 & 20 & 10 & 60 & 90 \\
\hline \multirow{3}{*}{ Orion A } & Both $(70)$ & 89 & 87 & 74 & 57 & 26 & 16 & 76 & 97 \\
\hline & Starless core $(24)$ & 79 & 88 & 63 & 50 & 13 & 13 & 63 & 96 \\
\hline & Protostellar core (46) & 93 & 87 & 80 & 61 & 33 & 17 & 83 & 98 \\
\hline \multirow{3}{*}{ Orion B } & Both (28) & 96 & 96 & 75 & 54 & 18 & 14 & 71 & 89 \\
\hline & Starless core $(10)$ & 90 & 100 & 60 & 60 & 10 & 20 & 70 & 90 \\
\hline & Protostellar core (18) & 100 & 94 & 83 & 50 & 22 & 11 & 72 & 89 \\
\hline \multirow{3}{*}{ Galactic plane } & Both (52) & 90 & 52 & 67 & 21 & $\cdots$ & 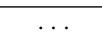 & 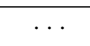 & $\cdots$ \\
\hline & Starless core (13) & 85 & 46 & 62 & 23 & $\ldots$ & $\ldots$ & $\ldots$ & $\ldots$ \\
\hline & Protostellar core (39) & 92 & 54 & 69 & 21 & $\ldots$ & $\ldots$ & $\cdots$ & $\ldots$ \\
\hline \multirow{3}{*}{ High latitudes } & Both (42) & 98 & 88 & 86 & 69 & 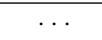 & 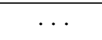 & $\ldots$ & $\ldots$ \\
\hline & Starless core $(6)$ & 100 & 83 & 83 & 83 & $\cdots$ & $\ldots$ & $\ldots$ & $\cdots$ \\
\hline & Protostellar core (36) & 97 & 89 & 86 & 67 & $\cdots$ & $\ldots$ & $\ldots$ & $\ldots$ \\
\hline
\end{tabular}

Noте- 'CCS-L' and 'CCS-H' represent the low and high transitions of CCS line, respectively. The numbers in parentheses indicate the total number of cores. The numbers represent the number of dense cores detected in the molecular line in percentages. All detection rates are inferred for sources whose peak temperature is higher than $3 \sigma$. There is no available data of CCS, $\mathrm{HC}_{3} \mathrm{~N}$, and $\mathrm{N}_{2} \mathrm{H}^{+}$lines for cores at other environments except for the Orion region.

Table 6 summarizes the detection rates of eight molecular lines in SCUBA-2 cores in different environments. Regarding the detection rates of $\mathrm{c}-\mathrm{C}_{3} \mathrm{H}_{2}, \mathrm{DNC}, \mathrm{HN}^{13} \mathrm{C}$, and $\mathrm{N}_{2} \mathrm{D}^{+}$lines observed towards all SCUBA-2 cores, it is found that the detection rates of all lines are higher than $49 \%$. In particular, the $\mathrm{c}^{-} \mathrm{C}_{3} \mathrm{H}_{2}$ (late-type in the cloud chemistry) line shows the highest detection rate $(91 \%)$ and is detected in almost all cores at high latitudes. The $\mathrm{HN}^{13} \mathrm{C}$ line is also detected at the highest rate in cores at high latitudes. The deuterated molecular lines DNC and $\mathrm{N}_{2} \mathrm{D}^{+}$are detected at the highest rate in cores in the Orion $\mathrm{B}$ and at high latitudes, respectively. All four lines tend to be more detected in protostellar cores than in starless cores

Regarding the detection rates of $\mathrm{CCS}-\mathrm{L} / \mathrm{H}, \mathrm{HC}_{3} \mathrm{~N}$, and $\mathrm{N}_{2} \mathrm{H}^{+}$lines that were only observed towards cores in the $\lambda$ Orionis, Orion $\mathrm{A}$, and $\mathrm{B}$, it is found that, overall speaking, the $\mathrm{N}_{2} \mathrm{H}^{+}$(late-type molecule) and $\mathrm{HC}_{3} \mathrm{~N}$ lines show high detection rates $(\geq 47 \%)$, whereas CCS lines, which trace the early phase in the cloud chemistry, show low detection rates $(\leq 26 \%)$. Among the three environments, all four lines are detected the highest in cores in the Orion $\mathrm{A}$, and the CCS- $\mathrm{L}, \mathrm{HC}_{3} \mathrm{~N}$, and $\mathrm{N}_{2} \mathrm{H}^{+}$ lines tend to be more detected in protostellar cores than in starless cores, whereas the CCS-H line tends to be more detected in starless cores than in protostellar cores.

In summary, both the low detection rate of early-type molecular lines and the high detection rate of late-type molecular lines and deuterated molecular lines suggest that most of the SCUBA-2 cores are chemically evolved.

\subsection{Beam dilution effect on distant SCUBA-2 cores}

Among the four column-density ratios estimated in Section 2.6, the column-density ratio of $\mathrm{DNC} / \mathrm{HN}^{13} \mathrm{C}$ is available for cores in all environments, but the three other column-density ratios, including $\mathrm{N}_{2} \mathrm{H}^{+}$molecules, are available only for cores in the $\lambda$ Orionis, Orion A, and B. To estimate the column-density ratio, we assume that the beam filling factor is the same for both the emission lines. However, because the SCUBA-2 cores are located at very different distances ranging from $\sim 0.1 \mathrm{pc}$ to $\sim 10 \mathrm{kpc}$, our telescope observes them with very different linear beam sizes. Figure 5 (a) shows the integratedintensity ratio of $\mathrm{DNC} / \mathrm{HN}^{13} \mathrm{C}$ against the linear beam size in pc. The ratio does not increase or decrease significantly for a beam size of $<0.1$ pc but decreases with increasing beam size for $>0.1$ pc with a power-law index of -1 . Because dense cores have a typical size of $0.1 \mathrm{pc}$, distant cores observed with larger beam size can be affected by different beam dilution between the two lines; DNC is beam-diluted while beam dilution for $\mathrm{HN}^{13} \mathrm{C}$ is much weaker. In other words, larger linear beams for distant cores tend to involve the outer, less-dense region having low deuterium fraction. A linear beam size of 19 arcsec corresponds to $0.1 \mathrm{pc}$ at a distance of 1.1 

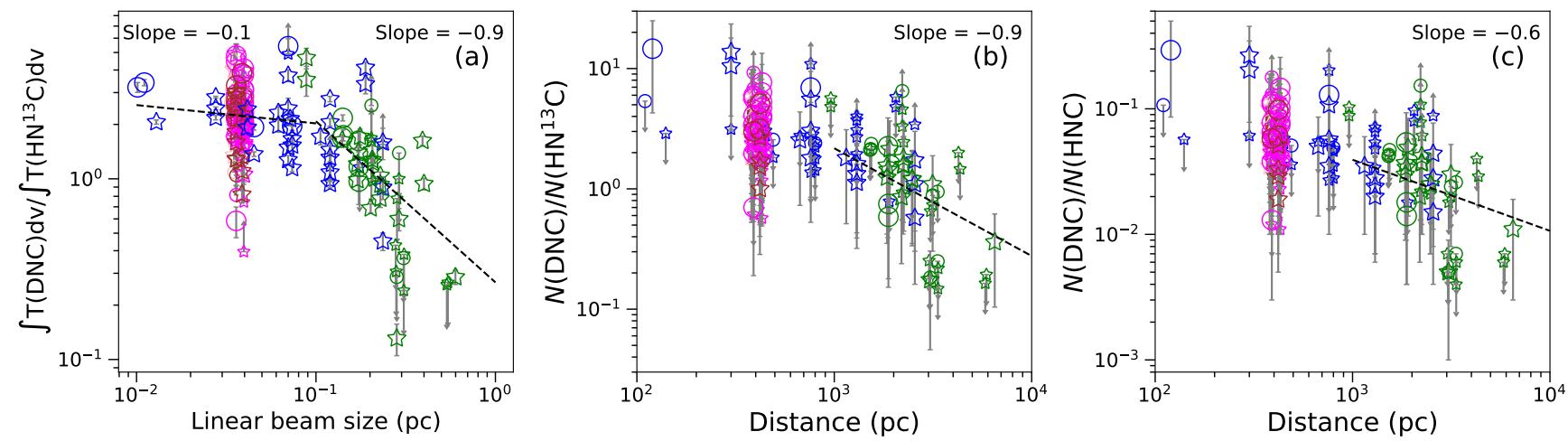

Figure 5. (a) The integrated-intensity ratio of $\mathrm{DNC}$ to $\mathrm{HN}^{13} \mathrm{C}$ is plotted against the linear beam size. (b) The column-density ratio of $\mathrm{DNC} / \mathrm{HN}^{13} \mathrm{C}$ is plotted against distance. (c) The same as (b) but for DNC/HNC. The color symbols represent SCUBA-2 cores in the $\lambda$ Orionis (pink), Orion A (magenta), Orion B (brown), Galactic plane (green), and at high latitudes (blue). The circle and star symbols represent the starless and protostellar core, respectively. The large symbol with the error bar represents the dense core where both values in the ratio are successfully estimated. The small symbol with the arrow represents the dense core with upper or lower limits. The dashed line with the slope value represents the least-squares fitting to the cores detected in both molecular lines. In panel (a), the fittings are made for linear beam sizes of $\leq 0.1$ pc and of $>0.1$ pc separately. In panels (b) and (c), the fittings are made for distances of $>1 \mathrm{kpc}$.

kpc. This differential beam dilution can lead to misinterpretations in the chemical properties of SCUBA-2 cores at different distances. We investigate whether it is the case for the columndensity ratio of $\mathrm{DNC} / \mathrm{HN}^{13} \mathrm{C}$ and $\mathrm{DNC} / \mathrm{HNC}$. In Figure 5 (b) and (c), we plot the column-density ratio against distance. The column-density ratio of DNC/HNC is derived by using the abundanceratio formula of ${ }^{12} \mathrm{C} /{ }^{13} \mathrm{C}$ as a function of Galactocentric distance obtained by Savage et al. (2002). Panels (b) and (c) of Figure 5 show similar trends that both the column-density ratios decrease with increasing distance for distant cores $(>1 \mathrm{kpc})$. It seems that the column-density ratio is seriously affected by differential beam dilution between the two lines for distant cores (distance $>1 \mathrm{kpc}$ ). Therefore, with our beam size, the column-density ratio seems reliable if distance is $<1$ kpc.

The percentage of protostellar cores $(65 \%)$ in the Orion region (distance $\sim 400 \mathrm{pc}$ ) is smaller than that of cores with distances of $2-10 \mathrm{kpc}$ in the Galactic plane $(81 \%)$. It is possible that the protostellar-core percentage increases with increasing distance. It is likely that the YSO sensitivity is shallower for distant cores. Furthermore, the beam dilution may merge weaker starless cores into brighter protostellar cores. In the Orion region, starless cores are half as intense as protostellar cores in the SCUBA-2 flux density. Figure 10 of Eden et al. (2019) shows that the percentage of the SCUBA-2 core detection decreases with increasing distance, which may suggest that the SCUBA-2 sensitivity becomes insufficient for weaker cores at larger distances.

To avoid distance-related issues, we start with the properties of cores in the three sub-regions of the Orion region with similar distances.

\subsection{Column-density ratios of $\mathrm{N}_{2} \mathrm{D}^{+} / \mathrm{N}_{2} \mathrm{H}^{+}$, $\mathrm{DNC} / \mathrm{HN}^{13} \mathrm{C}, \mathrm{N}_{2} \mathrm{H}^{+} / \mathrm{CCS}$, and $\mathrm{N}_{2} \mathrm{H}^{+} / \mathrm{HC}_{3} \mathrm{~N}$ for \\ $S C U B A-2$ cores in $\lambda$ Orionis, Orion $A$, and $B$}

Figure 6 plots the column-density ratios of $\mathrm{N}_{2} \mathrm{D}^{+} / \mathrm{N}_{2} \mathrm{H}^{+}$, $\mathrm{N}_{2} \mathrm{H}^{+} / \mathrm{CCS}$, and $\mathrm{N}_{2} \mathrm{H}^{+} / \mathrm{HC}_{3} \mathrm{~N}$ against that of $\mathrm{DNC} /$ $\mathrm{HN}^{13} \mathrm{C}$ for starless/protostellar cores in the $\lambda$ Orionis, Orion A, and B. Table 7 summarizes the statistics of the column-density ratios. We consider only cores where both column densities are successfully estimated.

For the four column-density ratios, there is no systematic difference between starless and protostellar cores in the $\lambda$ Orionis, Orion A and B. This may indicate that cores in these three regions have similar chemical properties as a whole.

We examine the correlations between the four columndensity ratios using the correlation coefficient (C.C) derived by the Pearson product-moment correlation coefficient. This method estimates the strength of the relationship between the relative movements of two variables and provides a value between -1.0 and +1.0 . The values of +1.0 and -1.0 indicate a strong positive and negative correlation, respectively, and the value of 0 represents no correlation. The C.C. considers only cores of 7 or more in the number of samples where both column densities in the ratio are successfully calculated, but does not take into account outliers in the sample. For $N\left(\mathrm{~N}_{2} \mathrm{D}^{+}\right) / N\left(\mathrm{~N}_{2} \mathrm{H}^{+}\right)$and $N(\mathrm{DNC}) / N\left(\mathrm{HN}^{13} \mathrm{C}\right)$, cores in the Orion $\mathrm{A}$ show a positive correlation. 

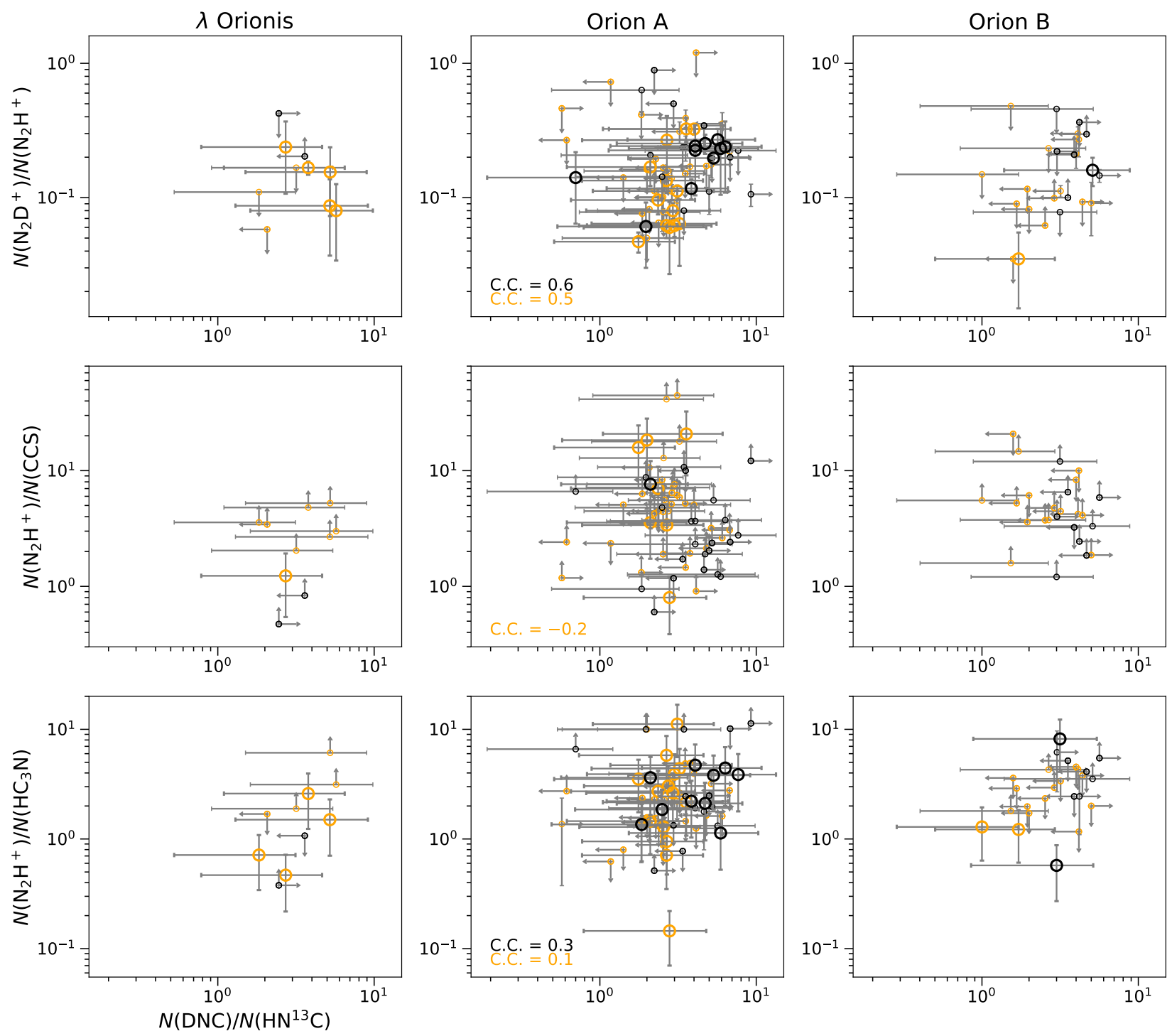

Figure 6. The column-density ratios of $\mathrm{N}_{2} \mathrm{D}^{+} / \mathrm{N}_{2} \mathrm{H}^{+}, \mathrm{N}_{2} \mathrm{H}^{+} / \mathrm{CCS}$, and $\mathrm{N}_{2} \mathrm{H}^{+} / \mathrm{HC}_{3} \mathrm{~N}$ are plotted against that of DNC/HN ${ }^{13} \mathrm{C}$ for SCUBA-2 cores in the $\lambda$ Orionis, Orion A, and B. The black and orange represent starless and protostellar cores, respectively. The large symbol with the error bar represents the dense core where both column densities in the ratio are successfully estimated. The small symbol with the arrow represents the dense core with either upper or lower limits. The C.C. represents the correlation coefficient; we show the C.C. only when both the pairing column densities are successfully estimated for seven or more dense cores. The C.C. does not take into account outliers in the sample.

This result suggests that $N\left(\mathrm{~N}_{2} \mathrm{D}^{+}\right) / N\left(\mathrm{~N}_{2} \mathrm{H}^{+}\right)$and $N(\mathrm{DNC}) / N\left(\mathrm{HN}^{13} \mathrm{C}\right)$ seem effective for $\mathrm{SCUBA}-2$ cores as chemical evolution tracers.

YSOs inside star-forming cores will have shocks and radiation and make the chemistry of protostellar cores more complex. In this paper, we defer our discussion on protostellar cores to our future papers, and concentrate on the characteristics of starless cores.

\subsection{Column-density ratio against $\mathrm{H}_{2}$ column density and dust temperature}

The $\mathrm{H}_{2}$ column density and dust temperature in the central region of the core can be used as the indicator of core evolution because the density increases and the temperature decreases throughout the starless core evolution (Shirley et al. 2005; Aikawa et al. 2008). We investigate whether $\mathrm{D} / \mathrm{H}$ has any correlation with the dust temperature and $\mathrm{H}_{2}$ column density for local $(<1 \mathrm{kpc})$ starless cores in different environments using the correlation coefficient. Figure 7 shows the column-density ratio of $\mathrm{DNC} / \mathrm{HN}^{13} \mathrm{C}$ and $\mathrm{N}_{2} \mathrm{D}^{+} / \mathrm{N}_{2} \mathrm{H}^{+}$against the dust temperature and $\mathrm{H}_{2}$ column density. Cores in the Galactic plane are excluded because all of the cores are more distant than $1 \mathrm{kpc}$.

For $\mathrm{D} / \mathrm{H}$ against the dust temperature and against the $\mathrm{H}_{2}$ column density, there seems to be no apparent 

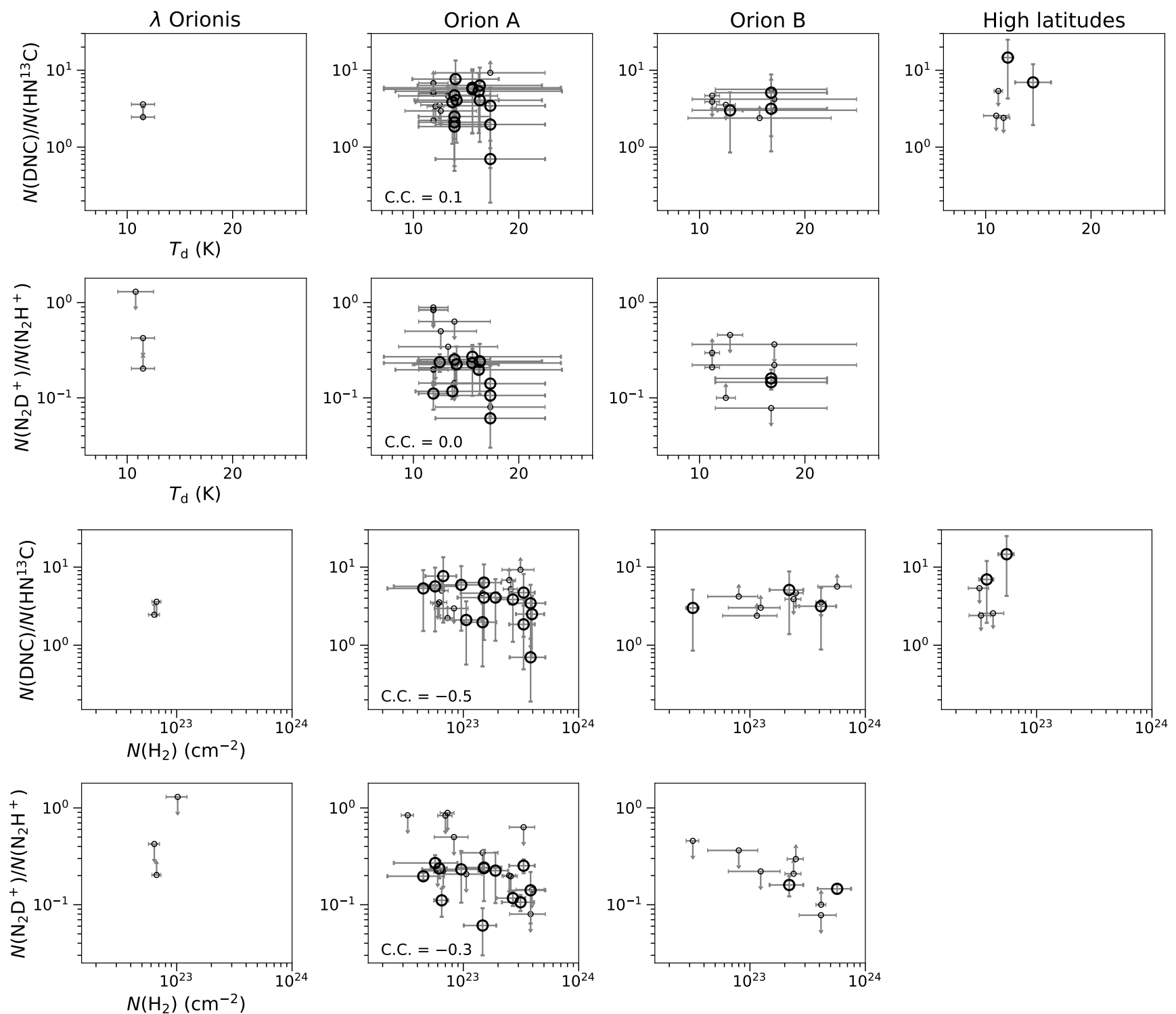

Figure 7. The column-density ratios of $\mathrm{DNC} / \mathrm{HN}^{13} \mathrm{C}$ and $\mathrm{N}_{2} \mathrm{D}^{+} / \mathrm{N}_{2} \mathrm{H}^{+}$are plotted against the dust temperature and $\mathrm{H}_{2}$ column density for local $(<1 \mathrm{kpc})$ starless cores in different environments. The meanings of the symbols and C.C. are the same as those used in Figure 6.
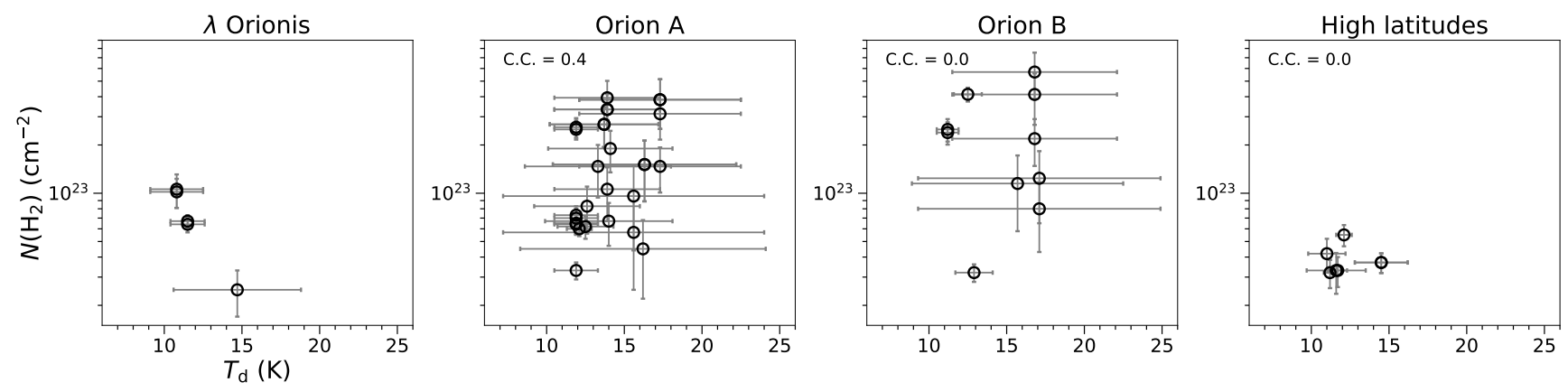

Figure 8. The $\mathrm{H}_{2}$ column density is plotted against dust temperature for local $(<1 \mathrm{kpc})$ starless cores in different environments. The meanings of the symbols and C.C. are the same as those used in Figure 6. 


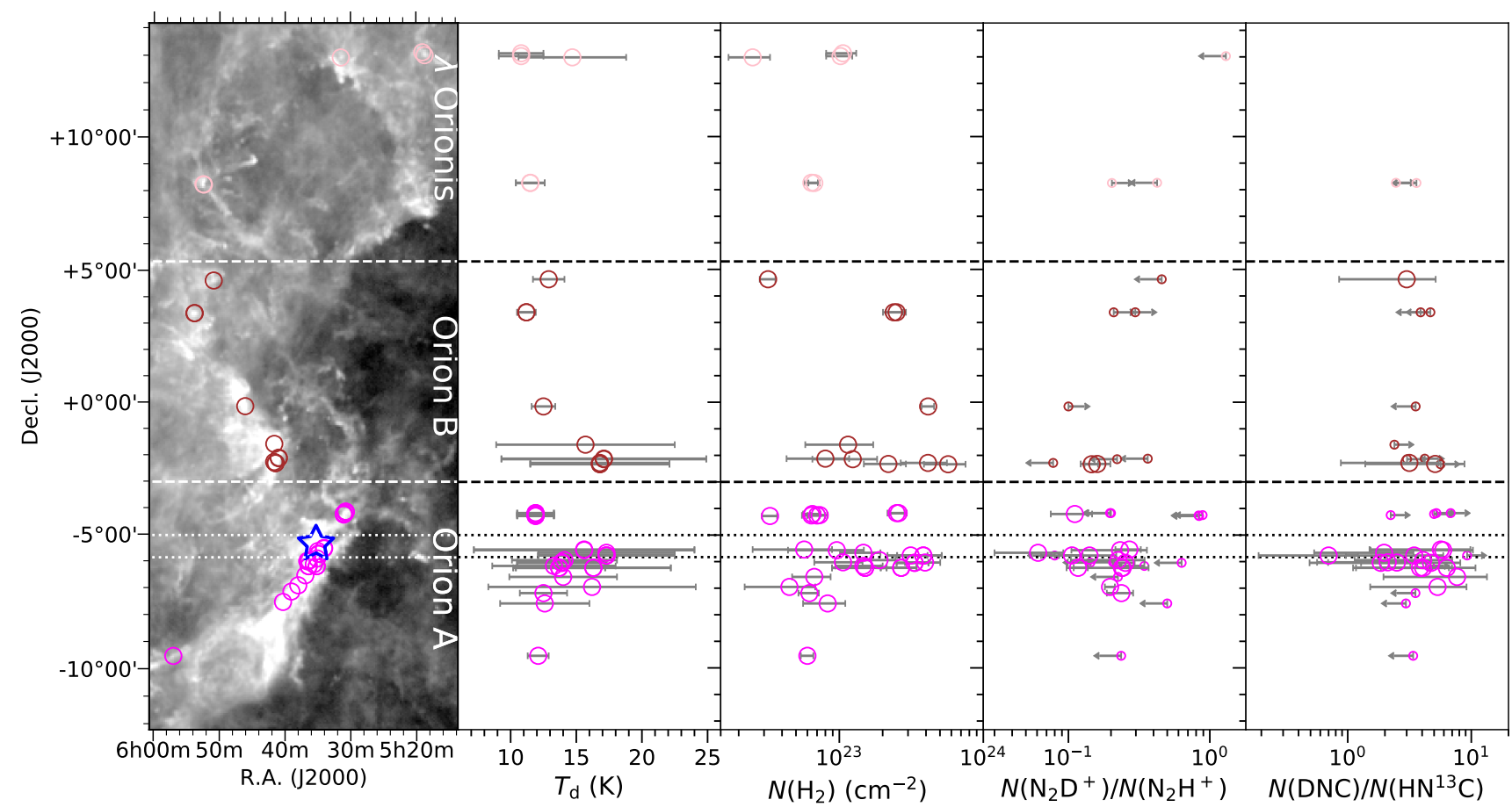

Figure 9. Spatial distribution, dust temperature, $\mathrm{H}_{2}$ column density, $N\left(\mathrm{~N}_{2} \mathrm{D}^{+}\right) / N\left(\mathrm{~N}_{2} \mathrm{H}^{+}\right)$, and $N(\mathrm{DNC}) / N\left(\mathrm{HN}{ }^{13} \mathrm{C}\right)$ against declination for starless cores in the Orion region. The large symbol with errorbar and the small symbol with an arrow are the same as those used in Figure 6. The background image in the left panel is the Planck $850 \mu$ m dust continuum map. The blue star symbol in the left panel represents the position of the Orion Nebula (R.A., Decl. $=05^{h} 35^{m} 17.3^{s},-05^{\circ} 23^{\prime} 28^{\prime \prime}$ ). The dashed horizontal lines represent the boundaries of $\lambda$ Orionis, Orion B, and Orion A. The dotted horizontal lines represent the boundaries of the potential impact zone of the Orion Nebula on the declination.

correlation. This is likely due to the fact that the spatial resolution of the Planck telescope ( $5^{\prime}$ corresponds to $\sim 0.5 \mathrm{pc}$ at a distance of $\sim 400 \mathrm{pc}$ ) is too low to trace the variation of temperature or density. Figure 8 plots the $\mathrm{H}_{2}$ column density as a function of dust temperature for local starless cores in different environments, and shows no clear correlation between the two quantities. No clear trend is seen probably because the resolution is too low.

\subsection{Variation of $D / H$, dust temperature, $H_{2}$ column density against declination for starless cores in the $\lambda$ Orionis, Orion $A$, and $B$}

In the Orion region, shell/ring-shaped structure $\lambda$ Orionis, filamentary clouds Orion A and B are extensively distributed along the declination, as shown in Figure 2. For starless cores in the $\lambda$ Orionis, Orion A, and B, we examine whether there is any variation in dust temperature, $\mathrm{H}_{2}$ column density, and $\mathrm{D} / \mathrm{H}$ along the declination. Figure 9 shows the spatial distribution, dust temperature, $\mathrm{H}_{2}$ column density, $N\left(\mathrm{~N}_{2} \mathrm{D}^{+}\right) / N\left(\mathrm{~N}_{2} \mathrm{H}^{+}\right)$, and $N(\mathrm{DNC}) / N\left(\mathrm{HN}^{13} \mathrm{C}\right)$ with respect to declination. Along the declination, the dust temperature and $\mathrm{H}_{2}$ column density appear to increase toward a declination of $\sim-4^{\circ}$ to $-3^{\circ}$. We exclude cores with a declination from $-5^{\circ} 50^{\prime}$ to $-5^{\circ}$ strongly externally heated by the Orion
Nebula. Although we see slight increase in the dust temperature and $\mathrm{H}_{2}$ column density near the Orion Nebula, we do not see any systematic trend in the $\mathrm{D} / \mathrm{H}$ fraction.

\subsection{Comparison of deuterium fraction between starless cores and other cores}

We compare the deuterium fraction between starless cores and other cores reported in previous studies. The average $N\left(\mathrm{~N}_{2} \mathrm{D}^{+}\right) / N\left(\mathrm{~N}_{2} \mathrm{H}^{+}\right)$of starless cores in the Orion region $(0.2 \pm 0.1)$ is found to be lower than that of PGCCs (0.5) reported in Tatematsu et al. (2017), which is similar to that of low-mass starless cores (0.1, Crapsi et al. 2005), and higher than that of massive protostellar IRAS cores (0.01, Fontani et al. 2006). If we assume ${ }^{12} \mathrm{C} /{ }^{13} \mathbf{C}=52$ (Savage et al. 2002), the average $N($ DNC) $/ N($ HNC) of starless cores in the Orion region $(\mathbf{0 . 0 8} \pm \mathbf{0 . 0 3})$ seems to be similar to those of 13 PGCCs (0.08) found in Tatematsu et al. (2017) and protostellar cores in the Perseus molecular cloud (0.06) in Imai et al. (2018), but higher than that of low-mass starless cores (0.02) in Hirota \& Yamamoto (2006). Approximately $30 \%$ of the starless cores in the Orion region are found to have a $N(\mathrm{DNC}) / N(\mathrm{HNC})$ higher than the value of 0.05 for L1544 (Hirota et al. 2003; Hirota \& Yamamoto 2006), suggesting that they could be 
starless cores more evolved toward the beginning of star formation.

\section{DISCUSSION}

4.1. Identification of the dense cores on the verge of star formation in the Orion region using CEFs

The CEF is expressed in the form of CEF $=\log ([N(\mathrm{~A}) /$ $\left.N(\mathrm{~B})] /\left[N_{0}(\mathrm{~A}) / N_{0}(\mathrm{~B})\right]\right) \times d$ for molecules $\mathrm{A}$ and $\mathrm{B}$. $N(\mathrm{~A}) / N(\mathrm{~B})$ is the ratio of column densities inferred from observations, $N_{0}(\mathrm{~A}) / N_{0}(\mathrm{~B})$ is the ratio of column densities at the onset of star formation. The factor $d$ is defined so that all cores range from $\sim-100$ for starless cores to $\sim+100$ for star-forming cores through 0 for cores on the verge of star formation. In this paper, we derive the $\mathrm{CEF}$ for only starless cores to identify late starless cores using $N\left(\mathrm{~N}_{2} \mathrm{D}^{+}\right) / N\left(\mathrm{~N}_{2} \mathrm{H}^{+}\right)$and $N(\mathrm{DNC}) / N\left(\mathrm{HN}^{13} \mathrm{C}\right)$. For starless cores, $N_{0}(\mathrm{~A}) / N_{0}(\mathrm{~B})$ approximately corresponds to the maximum of the column-density ratio of $\mathrm{D} / \mathrm{H}$ molecules and $d$ is determined for all starless cores so that the $\mathrm{CEF}$ ranges from $\sim-100$ to $\sim 0$. Using two column-density ratios listed in Table 5, we determine $N_{0}$ and $d$. We consider only starless cores in the Orion region because they should be located at similar distances. Out of them, we consider only cores in which both column densities in the ratio are successfully estimated. We exclude cores externally affected by large-scale star-forming activities (e.g., Orion Nebula, See Figure 9). For the 16 starless cores in the Orion region for which columndensity ratios are successfully obtained, we first derive the CEF values using the definition of Tatematsu et al. (2017), which we call CEF1.0. The top panel of Figure 10 shows the CEF1.0 values of the Orion cores. We also plot local $(<1$ kpc) cores studied in Tatematsu et al. (2017). CEF1.0 ranges from $\sim-75$ to $\sim 50$, which is different from the original definition of the CEF. For example, L1544, known as a gravitational collapsing prestellar core (Tafalla et al. 1998), has a CEF1.0 close to zero, which is reasonable. However, half of the starless Orion cores have positive CEF1.0, suggesting that CEF1.0 of Tatematsu et al. (2017) needs to be updated.

Using both samples of our starless Orion cores and the local cores from Tatematsu et al. (2017), we determine new CEF2.0 equations in the following forms:

$$
\begin{aligned}
& \mathrm{CEF} 2.0\left(\mathrm{~N}_{2} \mathrm{D}^{+}\right)=\log \left(\frac{N\left(\mathrm{~N}_{2} \mathrm{D}^{+}\right) / N\left(\mathrm{~N}_{2} \mathrm{H}^{+}\right)}{0.56}\right) \times 59, \\
& \mathrm{CEF} 2.0(\mathrm{DNC})=\log \left(\frac{N(\mathrm{DNC}) / N\left(\mathrm{HN}^{13} \mathrm{C}\right)}{9.3}\right) \times 87 .
\end{aligned}
$$

We search for $N_{0}(\mathbf{A}) / N_{0}(\mathbf{B})$ and $d$ for CEF2.0 $\left(\mathbf{N}_{2} \mathbf{D}^{+}\right)$ and CEF2.0(DNC) that minimizes the root mean squares of CEF2.0 $\left(\mathrm{N}_{2} \mathrm{D}^{+}\right)-\mathrm{CEF} 2.0(\mathrm{DNC})$ while satisfying a condition that the minimum
CEF2.0(average) is -100 , and the maximum CEF2.0(average) is 0 for the starless cores. The reason for finding $N_{0}(\mathbf{A}) / N_{0}(\mathbf{B})$ and $d$ in this manner is to illustrate chemical evolution as simple as possible because different pairs of molecules may show different characteristics. The uncertainty of the CEF is estimated through propagation from the error of the column-density ratio. The derived CEF2.0 is shown in the bottom panel of Figure 10, and values for the starless Orion cores are listed in Table 8.

The CEF1.0 of Tatematsu et al. (2017) was constructed on the basis of 15 nearby low-mass starless cores with kinetic temperatures of $10-20 \mathrm{~K}$ observed at spatial resolutions of $0.015-0.05 \mathrm{pc}$. In the present study, the CEF2.0 is constructed by adding the data of the 16 starless GMC cores in the Orion region to the existing samples having similar ranges of temperature and spatial resolution. Part of the origin of differences between the two CEF versions is probably due to the different range of evolutionary stages. For instance, Tatematsu et al. (2017) used cores of a broad range of different environments while the present study investigates one region where environmental differences may be smaller. Indeed, the detection rate of CCS is low in the Orion cores (Section 3.1), suggesting that they are chemically evolved. Furthermore, we used SCUBA-2 cores for observations, which are possibly biased to evolved cores having steeper radial intensity distribution. It is likely that these facts make the CEF2.0 values of starless Orion cores closer to zero. Probably our new CEF2.0 can be used more reliably if the telescope beam size is $\lesssim 0.1$ pc.

The bottom panel of Figure 10 shows the CEF2.0 of the 16 starless cores in the Orion region and the samples of Tatematsu et al. (2017) aligned in ascending numeric order of the CEF2.0. For the starless Orion cores, the CEF2.0 ranges from $\sim-60$ (G209.77-19.40East3) to $\sim 0$ (G210.37-19.53North). Compared to the CEF2.0 for L1544, we judge that at least eight starless cores have CEF2.0 close to zero $(\geq-33)$, suggesting that they are late starless cores on the verge of star formation. Figure 11 shows their spatial distribution on the Planck $850 \mu \mathrm{m}$ dust continuum map. They are mostly located in the south of Orion KL, at Decl. (J2000) $=-7.2$ to -5.9. We suggest that these cores are good targets for studying the initial conditions of star formation. G211.16-19.33North3, one of these starless cores having CEF2.0 close to zero, and the starforming Orion core G210.82-19.47North1 having bright $\mathrm{N}_{2} \mathrm{D}^{+}$emission were recently observed by Tatematsu et al. (2020) with the ALMA ACA interferometer. 

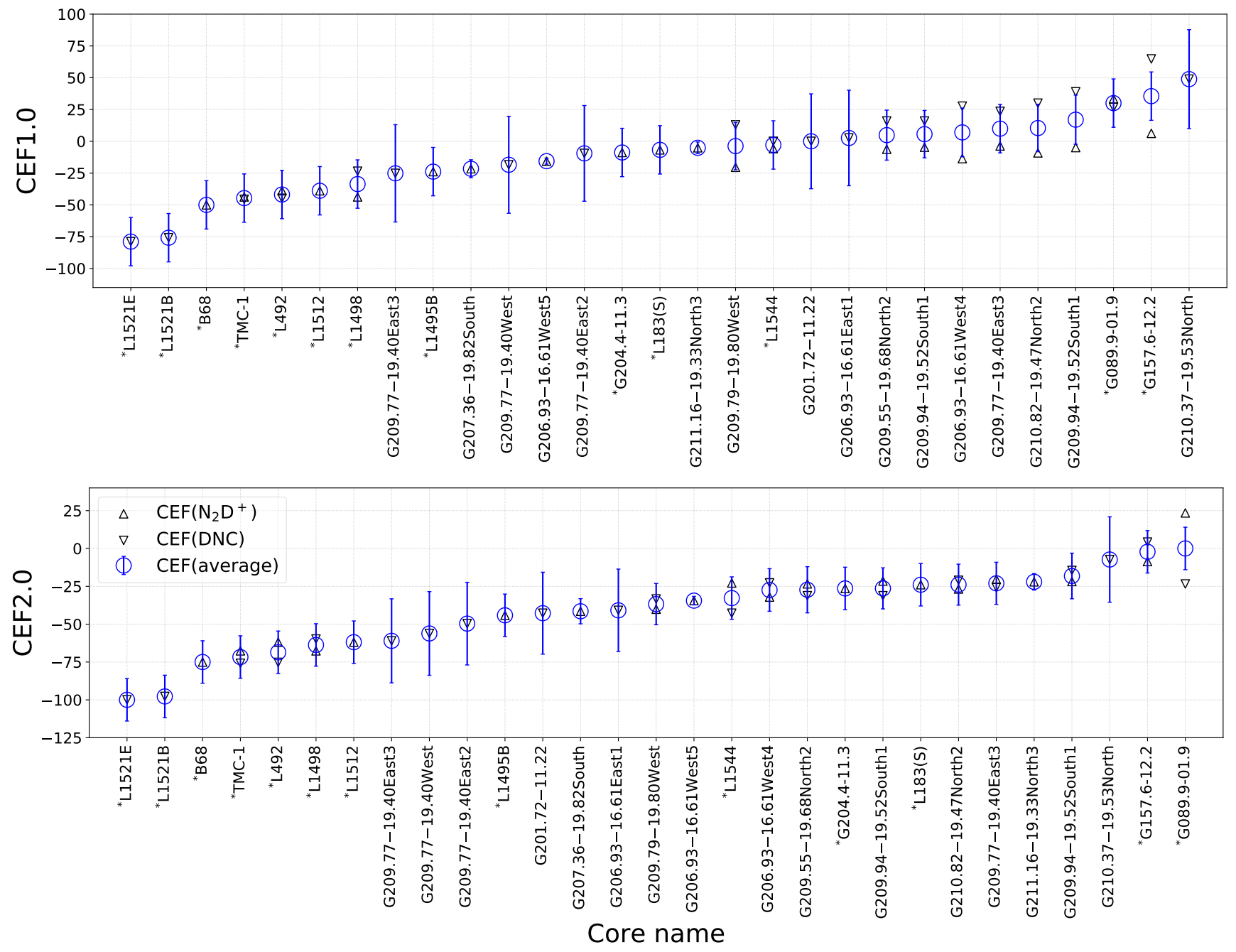

Figure 10. Chemical Evolutionary Factor (CEF) for starless cores in the Orion region and for local ( $<\mathbf{1}$ kpc) starless cores studied in Tatematsu et al. (2017) in ascending numeric order of the average CEF. The top and bottom panels show CEF1.0 derived by the equations in Tatematsu et al. (2017) and CEF2.0 by our study, respectively. The source name with the asterisk represents the cores taken from Tatematsu et al. (2017).

Regarding the number of the starless cores with successful CEF2.0 estimation, the Orion A sub-region has the largest number. The percentages of the starless cores with successful CEF2.0 estimation are $43 \%$ and $40 \%$ for Orion $\mathrm{A}$ and $\mathrm{B}$, respectively, which are very similar. $\lambda$ Orionis has no core with successful CEF2.0, which is natural because the number of SCUBA2 cores is small. We see no difference among the Orion sub-regions in terms of the success rate of CEF2.0 estimation.

We investigate whether there is any variation in the physical properties of starless cores when the average CEF approaches zero. Figure 12 shows the diagrams for $\mathrm{H}_{2}$ column density, dust temperature, and Mach number as a function of the average CEF2.0. We simply compare the mean values of the physical properties for two groups separated by an average CEF2.0 of
-33: early starless phase (CEF2.0 $<-33$ ) and late starless phase $(-33 \leq$ CEF2.0). When starless cores evolve, three physical properties do not seem to increase or decrease significantly. The lack of trends in the $\mathrm{H}_{2}$ column density and dust temperature appear to be inconsistent with the prediction of the core evolution model (e.g., Shirley et al. 2005; Aikawa et al. 2008). These discrepancies may be due to the reasons discussed in Section 3.4. The lack of a trend in the Mach number may suggest that the turbulence dissipation in the dense region of starless core is not required for the onset of star formation, because turbulent dissipation is regarded as one possibility to change a stable core to an unstable one (e.g., Nakano 1998). Other mechanisms may be required to change stable cores to unstable ones (e.g., accretion flow of gas; Gómez et al. 2007). Indeed, in G211.16-19.33North3, Tatematsu et al. (2020) obtained a hint of gas accretion onto one 
Table 8. Chemical Evolutionary Factor (CEF2.0) for starless cores in the Orion region.

\begin{tabular}{lccc}
\hline \hline \multicolumn{1}{c}{ SCUBA-2 core } & CEF2.0 $\left(\mathrm{N}_{2} \mathrm{D}^{+}\right)$ & CEF2.0(DNC) & CEF2.0(average) \\
\hline G209.77-19.40East3 & $\ldots$ & $-61 \pm 28$ & $-61 \pm 28$ \\
G209.77-19.40West & $\ldots$ & $-56 \pm 28$ & $-56 \pm 28$ \\
G209.77-19.40East2 & $\ldots$ & $-50 \pm 27$ & $-50 \pm 27$ \\
G201.72-11.22 & $\ldots$ & $-43 \pm 27$ & $-43 \pm 27$ \\
G207.36-19.82South & $-41 \pm 8$ & $\ldots$ & $-41 \pm 8$ \\
G206.93-16.61East1 & $\ldots$ & $-41 \pm 27$ & $-41 \pm 27$ \\
G209.79-19.80West & $-40 \pm 4$ & $-33 \pm 27$ & $-37 \pm 14$ \\
G206.93-16.61West5 & $-34 \pm 3$ & $\cdots$ & $-34 \pm 3$ \\
G206.93-16.61West4 & $-32 \pm 6$ & $-23 \pm 28$ & $-27 \pm 14$ \\
G209.55-19.68North2 & $-23 \pm 14$ & $-31 \pm 27$ & $-27 \pm 15$ \\
G209.94-19.52South1 & $-21 \pm 3$ & $-31 \pm 27$ & $-26 \pm 14$ \\
G210.82-19.47North2 & $-27 \pm 2$ & $-21 \pm 27$ & $-24 \pm 14$ \\
G209.77-19.40East3 & $-20 \pm 4$ & $-26 \pm 28$ & $-23 \pm 14$ \\
G211.16-19.33North3 & $-22 \pm 5$ & $\ldots$ & $-22 \pm 5$ \\
G209.94-19.52South1 & $-22 \pm 14$ & $-15 \pm 27$ & $-18 \pm 15$ \\
G210.37-19.53North & $\ldots$ & $-7 \pm 28$ & $-7 \pm 28$ \\
\hline
\end{tabular}

Note-Column 1: SCUBA-2 core name, Column 2-3: Chemical Evolutionary Factor (CEF2.0) for $\mathrm{N}_{2} \mathrm{D}^{+}$and $\mathrm{DNC}$ molecules, respectively, Column 4: average CEF 2.0. Three dots indicate no detection.

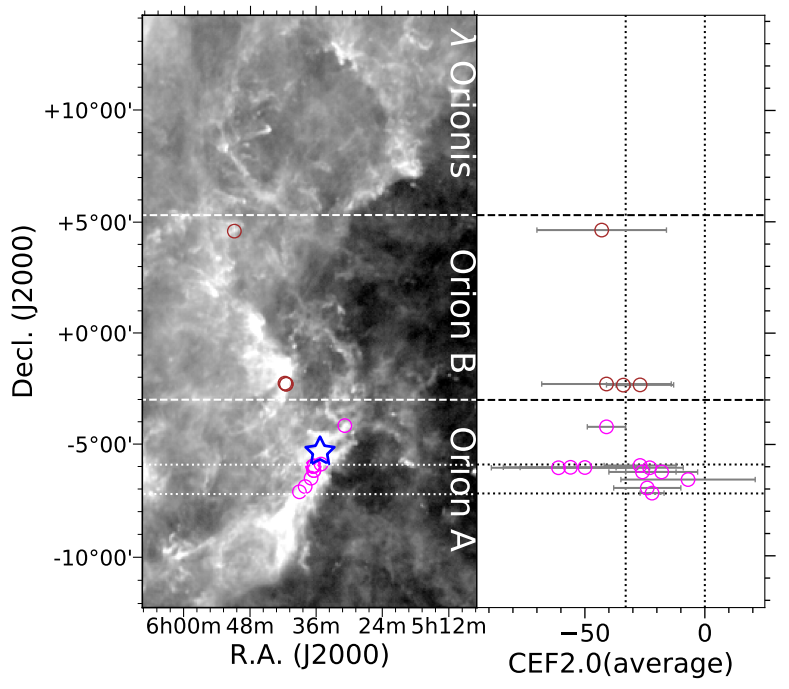

Figure 11. Same as Figure 9 but for the spatial distribution and average CEF2.0 against declination for starless cores in the Orion region. The vertical lines represent CEF2.0 of -33 and 0 . The horizontal dotted lines represent declination of -7.2 and -5.9 .

sub-core inside, with the ALMA ACA interferometer. However, we cannot rule out the possibility of turbulence dissipation for the beginning of star formation completely because the angular resolution of our telescope may be insufficient, and systematic observations with higher angular resolution are needed.

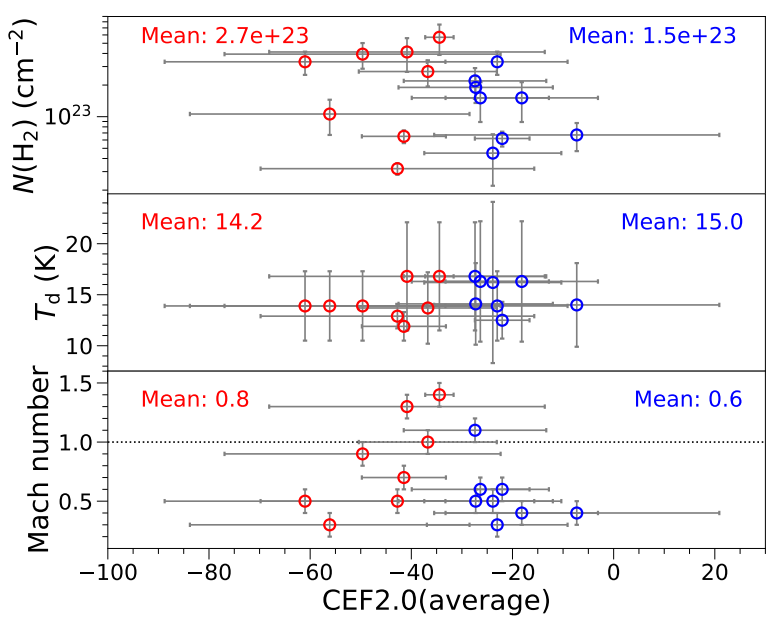

Figure 12. $\mathrm{H}_{2}$ column density, dust temperature, and Mach number against average CEF2.0 for starless cores in the Orion region. The red and blue represent starless cores with average CEF2.0 of $<-33$ and $\geq-33$, respectively.

\subsection{Identification of candidates for late starless cores in the environments other than the Orion}

For most of starless cores in the environments other than the Orion region, it is difficult to establish the CEF 2.0 because of largely different linear beam sizes (Section 3.2). Moreover, only $N(\mathrm{DNC}) / N\left(\mathrm{HN}^{13} \mathrm{C}\right)$ is available for these environments. Taking this into account, we look for late starless core candidates with both high $N(\mathrm{DNC}) / N\left(\mathrm{HN}^{13} \mathrm{C}\right)$ and $\mathrm{N}_{2} \mathrm{D}^{+}$detection among local $(<1 \mathrm{kpc})$ starless cores in two environments. All starless cores in the Galactic plane are more distant than $1 \mathrm{kpc}$. Two cores (SCOPEG001.37+20.95, SCOPEG202.32+02.53) at high latitudes are found to have high $N(\mathrm{DNC}) /$ $N\left(\mathrm{HN}^{13} \mathrm{C}\right)$ and detection in $\mathrm{N}_{2} \mathrm{D}^{+}$line. Among these cores, the highest $N(\mathrm{DNC}) / N\left(\mathrm{HN}^{13} \mathrm{C}\right)$ is found in SCOPEG001.37+ 20.95 (14.6).

\section{SUMMARY}

We conducted a molecular line survey of 207 SCUBA2 cores embedded in the Planck Galactic Cold Clumps with the Nobeyama 45-m telescope in the $\mathrm{N}_{2} \mathrm{D}^{+} J=$ $1-0, \mathrm{~N}_{2} \mathrm{H}^{+} J=1-0$, DNC $J=1-0, \mathrm{HN}^{13} \mathrm{C} J=$ $1-0, \operatorname{CCS} J_{N}=7_{6}-6_{5}, \operatorname{CCS} J_{N}=8_{7}-7_{6}, \mathrm{HC}_{3} \mathrm{~N}$ $J=9-8$, and $c-\mathrm{C}_{3} \mathrm{H}_{2} J_{K_{a} K_{c}}=22_{12}-1_{01}$ lines to identify dense cores on the verge of star formation in five different environments ( $\lambda$ Orionis, Orion A, B, Galactic plane, and high latitudes). The main results are summarized as follows:

- A total of 207 SCUBA-2 cores are classified into 58 starless cores (candidates) and 149 protostellar cores (candidates). They consist of 5 starless cores and 10 protostellar cores in the $\lambda$ Orionis, 24 and 46 in the Orion A, 10 and 18 in the Orion B, 13 
and 39 in the Galactic plane, 6 and 36 at high latitudes. Among the total 207 SCUBA-2 cores, starless cores occupy $\sim 28 \%$.

- The detection rates of early-type molecules (CCS) are low and the detection rates of late-type molecules $\left(\mathrm{c}-\mathrm{C}_{3} \mathrm{H}_{2}, \mathrm{~N}_{2} \mathrm{H}^{+}\right)$and the deuterated molecules (DNC, $\mathrm{N}_{2} \mathrm{D}^{+}$) are high, suggesting that most of the SCUBA-2 cores are chemically evolved.

- The integrated-intensity ratio and columndensity ratio of $\mathrm{DNC}$ to $\mathrm{HN}^{13} \mathrm{C}$ (HNC) tend to decrease with increasing distance $(>1 \mathrm{kpc}$ correspond to linear beam sizes of $>0.1 \mathrm{pc})$. This suggests that the deuterium fraction suffers from differential beam dilution between the two lines for distant cores $(>1$ kpc).

- For starless and protostellar cores in the $\lambda$ Orionis, Orion $\mathrm{A}$, and $\mathrm{B}$, the column-density ratios of $\mathrm{N}_{2} \mathrm{D}^{+} / \mathrm{N}_{2} \mathrm{H}^{+}$, DNC/HN ${ }^{13} \mathrm{C}, \mathrm{N}_{2} \mathrm{H}^{+} / \mathrm{CCS}$, and $\mathrm{N}_{2} \mathrm{H}^{+} / \mathrm{HC}_{3} \mathrm{~N}$ are similar. This suggests that cores in these three regions have similar chemical properties. Between four column-density ratios, $N(\mathrm{DNC}) / N\left(\mathrm{HN}^{13} \mathrm{C}\right)$ and $N\left(\mathrm{~N}_{2} \mathrm{D}^{+}\right) / N\left(\mathrm{~N}_{2} \mathrm{H}^{+}\right)$ have a positive correlation, suggesting that the two ratios could act as better tracers for core chemical evolution.

- In the Orion region, at least eight starless cores are identified as dense cores on the verge of star formation using the chemical evolution factor (CEF2.0) built on the basis of $N\left(\mathrm{~N}_{2} \mathrm{D}^{+}\right) / N\left(\mathrm{~N}_{2} \mathrm{H}^{+}\right)$and $N(\mathrm{DNC}) / N\left(\mathrm{HN}^{13} \mathrm{C}\right)$. At high latitudes, at least two starless cores are identified to be close to the beginning of star formation on the basis of high $N(\mathrm{DNC}) / N\left(\mathrm{HN}^{13} \mathrm{C}\right)$ and $\mathrm{N}_{2} \mathrm{D}^{+}$line detection. For starless cores in the Orion region, when starless cores evolve, the Mach number does not increase or decrease, which may indicate that the dissipation of turbulence in the dense region of the cores may not be an important mechanism for the onset of star formation as judged from observations with a beam size of $0.04 \mathrm{pc}$.

SCUBA-2 cores associated with high/low-mass starforming regions from the Galactic plane to high latitudes are located at highly different distances. For the study of the properties of dense cores at the constant spatial resolution with a single telescope, we need a set of observations to circumvent the beam dilution effect. In addition, for starless cores close to the onset of star formation found in this study, mapping observations are necessary to investigate whether they are gravitationally bound.

KT acknowledges Kouji Ohta for discussion. PS is partially supported by a Grant-in-Aid for Scientific Research (KAKENHI Number 18H01259) of Japan Society for the Promotion of Science (JSPS). JHH thanks the National Natural Science Foundation of China under grant Nos. 11873086 and U1631237 and support by the Yunnan Province of China (No.2017HC018). This work is sponsored (in part) by the Chinese Academy of Sciences (CAS), through a grant to the CAS South America Center for Astronomy (CASSACA) in Santiago, Chile. K. W. acknowledges support by the National Key Research and Development Program of China (2017YFA0402702, 2019YFA0405100), the National Science Foundation of China (11973013, 11721303), and the starting grant at the Kavli Institute for Astronomy and Astrophysics, Peking University (7101502287).

\section{Facilities: No:45m}

Software: astropy (Astropy Collaboration et al. 2013, 2018), GILDAS (Pety 2005; Gildas Team 2013), NEWSTAR

\section{REFERENCES}

Aidelman, Y., Cidale, L. S., Zorec, J., \& Panei, J. A. 2018, A\&A, 610, A30

Aikawa, Y., Herbst, E., Roberts, H., \& Caselli, P. 2005, ApJ, 620, 330

Aikawa, Y., Ohashi, N., \& Herbst, E. 2003, ApJ, 593, 906

Aikawa, Y., Ohashi, N., Inutsuka, S.-i., Herbst, E., \&

Takakuwa, S. 2001, ApJ, 552, 639

Aikawa, Y., Wakelam, V., Garrod, R. T., \& Herbst, E. 2008, ApJ, 674, 984

Anderson, T. G., Dixon, T. A., Piltch, N. D., et al. 1977, ApJL, 216, L85

André, P., Men'shchikov, A., Bontemps, S., et al. 2010, A\&A, 518, L102
Asayama, S., \& Nakajima, T. 2013, PASJ, 125, 213

Astropy Collaboration, Robitaille, T. P., Tollerud, E. J., et al. 2013, A\&A, 558, A33

Astropy Collaboration, Price-Whelan, A. M., Sipőcz, B. M., et al. 2018, AJ, 156, 123

Benson, P. J., Caselli, P., \& Myers, P. C. 1998, ApJ, 506, 743

Bergin, E. A., \& Tafalla, M. 2007, ARA\&A, 45, 339

Camargo, D., Bonatto, C., \& Bica, E. 2012, MNRAS, 423, 1940

Caselli, P. 2011, The Molecular Universe, 280, 19

Caselli, P., Benson, P. J., Myers, P. C., \& Tafalla, M. 2002a, ApJ, 572, 238 
Caselli, P., \& Myers, P. C. 1995, ApJ, 446, 665

Caselli, P., Myers, P. C., \& Thaddeus, P. 1995b, ApJL, 455, L77

Caselli, P., Walmsley, C. M., Zucconi, A., et al. 2002b, ApJ, 565,344

Chen, H.-R., Liu, S.-Y., Su, Y.-N., \& Wang, M.-Y. 2011, ApJ, 743, 196

Chen, H.-R., Liu, S.-Y., Su, Y.-N., \& Zhang, Q. 2010, ApJL, 713, L50

Contreras, Y., Sanhueza, P., Jackson, J. M., et al. 2018, ApJ, 861, 14

Crapsi, A., Caselli, P., Walmsley, C. M., et al. 2005, ApJ, 619,379

Crapsi, A., Caselli, P., Walmsley, M. C., \& Tafalla, M. 2007, A\&A, 470, 221

Cummins, S. E., Linke, R. A., \& Thaddeus, P. 1986, ApJS, 60,819

Dobashi, K. 2011, PASJ, 63, 1

Dunham, M. M., Allen, L. E., Evans, N. J. I., et al. 2015, ApJS, 220, 11

Eden, D. J., Liu, T., Kim, K.-T., et al. 2019, MNRAS, 485, 2895

Emprechtinger, M., Caselli, P., Volgenau, N. H., Stutzki, J., \& Wiedner, M. C. 2009, A\&A, 493, 89

Feng, S., Caselli, P., Wang, K., et al. 2019, ApJ, 883, 202

Fischera, J., \& Martin, P. G. 2012, A\&A, 547, A86

Fontani, F., Caselli, P., Crapsi, A., et al. 2006, A\&A, 460, 709

Fontani, F., Sakai, T., Furuya, K., et al. 2014, MNRAS, 440,448

Fontani, F., Palau, A., Caselli, P., et al. 2011, A\&A, 529, L7

Frerking, M. A., Langer, W. D., \& Wilson, R. W. 1979, ApJ, 232, L65

Friesen, R. K., Di Francesco, J., Shimajiri, Y., \& Takakuwa, S. 2010, ApJ, 708, 1002

Fuller, G. A., \& Myers, P. C. 1992, ApJ, 384, 523

Galli, P. A. B., Loinard, L., Ortiz-León, G. N., et al. 2018, ApJ, 859, 33

Gerner, T., Shirley, Y. L., Beuther, H., et al. 2015, A\&A, 579, A 80

Gildas Team 2013, GILDAS: Grenoble Image and Line

Data Analysis Software, ascl:1305.010

Gómez, G. C., Vazquez Semadeni, E., Shadmehri, M., \& Ballesteros Paredes, J. 2007, ApJ, 669, 1042

Hirota, T., Ikeda, M., \& Yamamoto, S. 2001, ApJ, 547, 814 -. 2003, ApJ, 594, 859

Hirota, T., \& Yamamoto, S. 2006, ApJ, 646, 258

Hirota, T., Ando, K., Bushimata, T., et al. 2008, PASJ, 60, 961

Humphreys, R. M. 1978, ApJ, 38, 309
Imai, M., Sakai, N., López-Sepulcre, A., et al. 2018, ApJ, 869,51

Juvela, M., Malinen, J., Montillaud, J., et al. 2018, A\&A, 614, A83

Juvela, M., Ristorcelli, I., Montier, L. A., et al. 2010, A\&A, 518, L93

Juvela, M., Juvela, M., Ristorcelli, I., et al. 2012, A\&A, 541, A12

Kamazaki, T., Okumura, S. K., Chikada, Y., et al. 2012, PASJ, 64, 29

Kim, G., Lee, C. W., Kim, J., et al. 2010, Journal of the Korean Astronomical Society, 43, 9

Könyves, V., André, P., Men'shchikov, A., et al. 2010, A\&A, 518, L106

Kounkel, M., Hartmann, L., Loinard, L., et al. 2017, ApJ, 834,142

Lada, C. J., Lombardi, M., \& Alves, J. F. 2009, ApJ, 703, 52

Lee, C. W., \& Myers, P. C. 1999, ApJS, 123, 233

Lee, J.-E., Bergin, E. A., \& Evans, N. J. I. 2004, ApJ, 617, 360

Lee, J.-E., Evans, N. J. I., Shirley, Y. L., \& Tatematsu, K. 2003, ApJ, 583, 789

Liu, T., Wu, Y., Mardones, D., et al. 2015, Publications of The Korean Astronomical Society, 30, 79

Liu, T., Kim, K.-T., Juvela, M., et al. 2018, ApJS, 234, 28

Lombardi, M., Lada, C. J., \& Alves, J. 2008, A\&A, 480, 785

Mangum, J. G., \& Shirley, Y. L. 2015, PASJ, 127, 266

Marton, G., Tóth, L. V., Paladini, R., et al. 2016, MNRAS, 458,3479

Marton, G., Ábrahám, P., Szegedi-Elek, E., et al. 2019, MNRAS, 487, 2522

McKee, C. F., \& Ostriker, E. C. 2007, ARA\&A, 45, 565

Megeath, S. T., Gutermuth, R., Muzerolle, J., et al. 2012, AJ, 144, 192

Millar, T. J., Bennett, A., \& Herbst, E. 1989, ApJ, 340, 906

Motte, F., Nony, T., Louvet, F., et al. 2018, Nature Astronomy, 2, 478

Myers, P. C. 1983, ApJ, 270, 105

Nakajima, T., Kimura, K., Nishimura, A., et al. 2013, PASJ, 125, 252

Nakano, T. 1998, ApJ, 494, 587

Ohashi, S., Sanhueza, P., Sakai, N., et al. 2018, ApJ, 856, 147

Ohashi, S., Tatematsu, K., Fujii, K., et al. 2016, PASJ, 68, 3

Ohashi, S., Tatematsu, K., Choi, M., et al. 2014, PASJ, 66, 119

Okabayashi, T., \& Tanimoto, M. 1993, JChPh, 99, 3268

Ortiz-León, G. N., Loinard, L., Dzib, S. A., et al. 2018, ApJ, 865, 73 
Pagani, L., Bacmann, A., Cabrit, S., \& Vastel, C. 2007, A\&A, 467, 179

Peretto, N., \& Fuller, G. A. 2009, A\&A, 505, 405

Perryman, M. A. C., Lindegren, L., Kovalevsky, J., et al. 1997, A\&A, 323, L49

Pety, J. 2005, SF2A-2005: Semaine De L'astrophysique Francaise, 721

Phillips, T. G., \& Vastel, C. 2003, SFChem 2002: Chemistry as a Diagnostic of Star Formation, 3

Pickett, H. M., Poynter, R. L., Cohen, E. A., \& and, M. D. S. 1998, J QUANT SPECTROSC RA, 60, 883

Pidopryhora, Y., Lockman, F. J., Dickey, J. M., \& Rupen, M. P. 2015, ApJS, 219, 16

Planck Collaboration, Ade, P. A. R., Aghanim, N., et al. 2011, A\&A, 536, A23

-. 2016, A\&A, 594, A28

Povich, M. S., Kuhn, M. A., Getman, K. V., et al. 2013, ApJS, 209, 31

Reid, M. J., Dame, T. M., Menten, K. M., \& Brunthaler, A. 2016, ApJ, 823, 77

Roberts, H., \& Millar, T. J. 2000, A\&A, 361, 388

Sakai, T., Sakai, N., Furuya, K., et al. 2012, ApJ, 747, 140

-. 2015, ApJ, 803, 70

Sakai, T., Yanagida, T., Furuya, K., et al. 2018, ApJ, 857, 35

Sanhueza, P., Jackson, J. M., Foster, J. B., et al. 2012, ApJ, 756, 60

Sanhueza, P., Jackson, J. M., Zhang, Q., et al. 2017, ApJ, 841, 97

Sanhueza, P., Contreras, Y., Wu, B., et al. 2019, ApJ, 886, 102

Savage, C., Apponi, A. J., Ziurys, L. M., et al. 2002, ApJ, 578,211

Shirley, Y. L., Nordhaus, M. K., Grcevich, J. M., et al. 2005, ApJ, 632, 982

Simon, R., Jackson, J. M., Rathborne, J. M., \& Chambers, E. T. 2006, ApJ, 639, 227
Straižys, V., Drew, J. E., \& Laugalys, V. 2010, Baltic Astronomy, 19, 169

Straižys, V., Maskoliūnas, M., Boyle, R. P., et al. 2014, MNRAS, 438, 1848

Straižys, V., Boyle, R. P., Zdanavičius, J., et al. 2018, A\&A, 611, A9

Sunada, K., Nakazato, T., Ikeda, N., et al. 2007, PASJ, 59, 1185

Suzuki, H., Yamamoto, S., Ohishi, M., et al. 1992, ApJ, 392,551

Tafalla, M., Mardones, D., Myers, P. C., et al. 1998, ApJ, 504,900

Tatematsu, K., Kandori, R., Umemoto, T., et al. 2008, PASJ, 60, 407

Tatematsu, K., Ohashi, S., Umemoto, T., et al. 2014, PASJ, 66, 16

Tatematsu, K., Liu, T., Ohashi, S., et al. 2017, ApJS, 228, 12

Tatematsu, K., Liu, T., Kim, G., et al. 2020, ApJ, 895, 119

Thaddeus, P., Vrtilek, J. M., \& Gottlieb, C. A. 1985, ApJ, 299, L63

van der Tak, F. F. S., Müller, H. S. P., Harding, M. E., \& Gauss, J. 2009, A\&A, 507, 347

Vastel, C., Phillips, T. G., \& Yoshida, H. 2004, ApJ, 606, L127

Wang, K., Zhang, Q., Testi, L., et al. 2014, MNRAS, 439, 3275

Wang, J., Shi, J., Pan, K., et al. 2016, MNRAS, 460, 3179

Ward-Thompson, D., Scott, P. F., Hills, R. E., \& André, P. 1994, MNRAS, 268, 276

Yamamoto, S., Saito, S., Kawaguchi, K., et al. 1990, ApJ, 361,318

Yi, H.-W., Lee, J.-E., Liu, T., et al. 2018, ApJS, 236, 51

Zhang, Q., Wang, K., Lu, X., et al. 2015, ApJ, 804, 141 
Table 1. Information of 207 SCUBA-2 cores embedded in Planck Galactic Cold Clumps

\begin{tabular}{|c|c|c|c|c|c|c|c|c|c|}
\hline SCUBA-2 core & & & YSO ass. & Dist. & Ref & $T_{\mathrm{d}}$ & $N\left(\mathrm{H}_{2}\right)$ & Env. & Comment \\
\hline & (hh:mm:ss.ss) & (dd:mm:ss.s) & & $(\mathrm{kpc})$ & & $(\mathrm{K})$ & $\left(\times 10^{23} \mathrm{~cm}^{-2}\right)$ & & \\
\hline (1) & $(2)$ & (3) & (4) & (5) & $(6)$ & (7) & $(8)$ & (9) & (10) \\
\hline G190.15-13.75North & $05: 19: 01.11$ & $13: 08: 05.8$ & starless & 0.38 & 11 & $10.8 \pm 1.7$ & $1.1 \pm 0.2$ & OL & PGCC G190.15-13.75, $\lambda$ Orionis \\
\hline G190.15-13.75South & $05: 18: 40.55$ & $13: 01: 41.6$ & starless & 0.38 & 11 & $10.8 \pm 1.7$ & $1.0 \pm 0.2$ & $\mathrm{OL}$ & PGCC G190.15-13.75, $\lambda$ Orionis \\
\hline G191.90-11.21North & $05: 31: 28.99$ & $12: 58: 55.0$ & starless & 0.38 & 11 & $14.7 \pm 4.1$ & $0.2 \pm 0.1$ & $\mathrm{OL}$ & PGCC G191.90-11.21, $\lambda$ Orionis \\
\hline G191.90-11.21South & $05: 31: 31.73$ & $12: 56: 15.0$ & protostellar & 0.38 & 11 & $14.7 \pm 4.1$ & $0.7 \pm 0.2$ & OL & WISE J053131.58+125615.2, PGCC G191.90-11.21, $\lambda$ Orionis \\
\hline G192.12-10.90North & $05: 33: 02.64$ & $12: 57: 53.6$ & protostellar & 0.38 & 11 & $13.1 \pm 1.5$ & $0.6 \pm 0.1$ & $\mathrm{OL}$ & WISE J053302.57+125810.1, PGCC G192.12-10.88, $\lambda$ Orionis \\
\hline G192.12-10.90South & $05: 32: 52.52$ & $12: 55: 08.6$ & protostellar & 0.38 & 11 & $13.1 \pm 1.5$ & $0.6 \pm 0.1$ & $\mathrm{OL}$ & WISE J053252.62+125509.0, PGCC G192.12-10.88, $\lambda$ Orionis \\
\hline G192.12-11.10 & $05: 32: 19.54$ & $12: 49: 40.2$ & protostellar & 0.38 & 11 & $13.3 \pm 3.0$ & $1.8 \pm 0.4$ & $\mathrm{OL}$ & WISE J053219.43+124942.5, PGCC G192.12-11.10, L1582, $\lambda$ Orionis \\
\hline G192.32-11.88North & $05: 29: 54.47$ & $12: 16: 56.0$ & protostellar & 0.38 & 11 & $17.3 \pm 6.0$ & $1.1 \pm 0.4$ & $\mathrm{OL}$ & [LZK2016] G192N, PGCC G192.32-11.88, L1581, $\lambda$ Orionis \\
\hline G192.32-11.88South & $05: 29: 54.74$ & $12: 16: 32.0$ & protostellar & 0.38 & 11 & $17.3 \pm 6.0$ & $0.9 \pm 0.4$ & OL & [LZK2016] G192S, PGCC G192.32-11.88, L1581, $\lambda$ Orionis \\
\hline G196.92-10.37 & $05: 44: 29.56$ & $09: 08: 50.2$ & protostellar & 0.38 & 11 & $14.8 \pm 0.4$ & $1.8 \pm 0.1$ & $\mathrm{OL}$ & [NFA2013] 2, PGCC G196.92-10.37, B35, L1594, $\lambda$ Orionis \\
\hline G198.69-09.12North1 & $05: 52: 29.61$ & $08: 15: 37.0$ & starless & 0.38 & 11 & $11.5 \pm 1.1$ & $0.6 \pm 0.1$ & $\mathrm{OL}$ & PGCC G198.69-9.12, L1598, $\lambda$ Orionis \\
\hline G198.69-09.12North2 & $05: 52: 25.30$ & $08: 15: 08.8$ & starless & 0.38 & 11 & $11.5 \pm 1.1$ & $0.7 \pm 0.1$ & $\mathrm{OL}$ & PGCC G198.69-9.12, L1598, $\lambda$ Orionis \\
\hline G198.69-09.12South & $05: 52: 23.66$ & $08: 13: 37.2$ & protostellar & 0.38 & 11 & $11.5 \pm 1.1$ & $1.5 \pm 0.2$ & OL & [MJR2015] 1386, PGCC G198.69-9.12, L1598, $\lambda$ Orionis \\
\hline G200.34-10.97North & $05: 49: 03.71$ & $05: 57: 55.7$ & protostellar & 0.38 & 11 & $13.5 \pm 0.9$ & $0.8 \pm 0.1$ & $\mathrm{OL}$ & WISE J054903.37+055757.9, PGCC G200.34-10.98, $\lambda$ Orionis \\
\hline G200.34-10.97South & $05: 49: 07.74$ & $05: 55: 36.2$ & protostellar & 0.38 & 11 & $13.5 \pm 0.9$ & $0.7 \pm 0.1$ & OL & WISE J054907.41+055539.6, PGCC G200.34-10.98, $\lambda$ Orionis \\
\hline G201.52-11.08 & $05: 50: 59.01$ & $04: 53: 53.1$ & protostellar & 0.39 & 7 & $13.6 \pm 1.3$ & $0.8 \pm 0.1$ & OB & WISE J055059.14+045349.5, PGCC G201.52-11.08, Orion B \\
\hline G201.72-11.22 & $05: 50: 54.53$ & $04: 37: 42.6$ & starless & 0.39 & 7 & $12.9 \pm 1.2$ & $0.3 \pm 0.1$ & OB & PGCC G201.72-11.22, Orion B \\
\hline G203.21-11.20East 1 & $05: 53: 51.11$ & $03: 23: 04.9$ & starless & 0.39 & 7 & $11.2 \pm 0.7$ & $2.4 \pm 0.4$ & $\mathrm{OB}$ & PGCC G203.21-11.20, Orion B \\
\hline G203.21-11.20East2 & $05: 53: 47.90$ & $03: 23: 08.9$ & starless & 0.39 & 7 & $11.2 \pm 0.7$ & $2.5 \pm 0.4$ & OB & PGCC G203.21-11.20, Orion B \\
\hline G203.21-11.20West1 & $05: 53: 42.83$ & $03: 22: 32.9$ & protostellar & 0.39 & 7 & $11.2 \pm 0.7$ & $2.7 \pm 0.3$ & OB & Herschel J055342.5+032236, PGCC G203.21-11.20, Orion B \\
\hline G203.21-11.20West2 & $05: 53: 39.62$ & $03: 22: 24.9$ & protostellar & 0.39 & 7 & $11.2 \pm 0.7$ & $3.2 \pm 0.4$ & OB & Herschel J055339.5+032225, PGCC G203.21-11.20, Orion B \\
\hline G204.4-11.3A2East & $05: 55: 38.43$ & $02: 11: 33.3$ & protostellar & 0.39 & 7 & $11.1 \pm 1.9$ & $4.7 \pm 0.9$ & $\mathrm{OB}$ & Herschel J055538.2+021135, PGCC G204.49-11.33, Orion B \\
\hline G204.4-11.3A2West & $05: 55: 35.49$ & $02: 11: 01.3$ & protostellar & 0.39 & 7 & $11.1 \pm 1.9$ & $3.5 \pm 0.6$ & OB & Herschel J055535.3+021103, PGCC G204.49-11.33, Orion B \\
\hline G205.46-14.56Middle1 & $05: 46: 09.65$ & $-00: 12: 12.9$ & protostellar & 0.39 & 7 & $12.5 \pm 0.9$ & $5.5 \pm 0.5$ & OB & HOPS 402, PGCC G205.46-14.56, L1630, Orion B \\
\hline G205.46-14.56Middle2 & $05: 46: 07.49$ & $-00: 12: 22.4$ & protostellar & 0.39 & 7 & $12.5 \pm 0.9$ & $4.7 \pm 0.4$ & OB & HOPS 401, PGCC G205.46-14.56, L1630, Orion B \\
\hline G205.46-14.56Middle3 & $05: 46: 07.37$ & $-00: 11: 53.4$ & protostellar & 0.39 & 7 & $12.5 \pm 0.9$ & $4.8 \pm 0.5$ & OB & SSV LDN 1630 61, PGCC G205.46-14.56, L1630, Orion B \\
\hline G205.46-14.56North3 & $05: 46: 08.06$ & $-00: 10: 43.6$ & protostellar & 0.39 & 7 & $12.5 \pm 0.9$ & $8.7 \pm 0.8$ & OB & HOPS 317, PGCC G205.46-14.56, L1630, Orion B \\
\hline G205.46-14.56North2 & $05: 46: 07.89$ & $-00: 10: 02.0$ & protostellar & 0.39 & 7 & $12.5 \pm 0.9$ & $5.5 \pm 0.5$ & OB & HOPS 387, PGCC G205.46-14.56, L1630, Orion B \\
\hline G205.46-14.56North1 & $05: 46: 05.49$ & $-00: 09: 32.4$ & starless & 0.39 & 7 & $12.5 \pm 0.9$ & $4.1 \pm 0.4$ & OB & PGCC G205.46-14.56, L1630, Orion B \\
\hline G205.46-14.56South1 & $05: 46: 07.11$ & $-00: 13: 34.6$ & protostellar & 0.39 & 7 & $12.5 \pm 0.9$ & $6.7 \pm 0.6$ & OB & HOPS 358, PGCC G205.46-14.56, L1630, Orion B \\
\hline G205.46-14.56South2 & $05: 46: 04.49$ & $-00: 14: 18.9$ & protostellar & 0.39 & 7 & $12.5 \pm 0.9$ & $4.8 \pm 0.5$ & OB & HOPS 385, PGCC G205.46-14.56, L1630, Orion B \\
\hline G205.46-14.56South3 & $05: 46: 03.54$ & $-00: 14: 49.3$ & protostellar & 0.39 & 7 & $12.5 \pm 0.9$ & $5.0 \pm 0.5$ & OB & HOPS 315, PGCC G205.46-14.56, L1630, Orion B \\
\hline G206.12-15.76 & $05: 42: 45.27$ & $-01: 16: 11.4$ & protostellar & 0.39 & 7 & $11.9 \pm 1.6$ & $2.6 \pm 0.4$ & OB & HOPS 400, PGCC G206.12-15.76, Orion B \\
\hline G206.21-16.17North & $05: 41: 39.28$ & $-01: 35: 52.9$ & starless & 0.39 & 7 & $15.7 \pm 6.8$ & $1.1 \pm 0.6$ & OB & PGCC G206.21-16.17, Orion B \\
\hline G206.69-16.60North & $05: 40: 58.08$ & $-02: 07: 28.3$ & starless & 0.42 & 7 & $17.1 \pm 7.8$ & $0.8 \pm 0.4$ & OB & PGCC G206.69-16.60, Orion B \\
\hline G206.69-16.60South & $05: 40: 58.62$ & $-02: 08: 40.7$ & starless & 0.42 & 7 & $17.1 \pm 7.8$ & $1.2 \pm 0.6$ & $\mathrm{OB}$ & PGCC G206.69-16.60, Orion B \\
\hline
\end{tabular}

Table 1 continued 
Table 1 (continued)

\begin{tabular}{|c|c|c|c|c|c|c|c|c|c|}
\hline SCUBA-2 core & $\begin{array}{l}\text { R.A.(J2000) } \\
\text { (hh:mm:ss.ss) }\end{array}$ & $\begin{array}{l}\text { Decl.(J2000) } \\
\text { (dd:mm:ss.s) }\end{array}$ & YSO ass. & $\begin{array}{l}\text { Dist. } \\
(\mathrm{kpc})\end{array}$ & Ref & $\begin{array}{l}T_{\mathrm{d}} \\
(\mathrm{K})\end{array}$ & $\begin{array}{c}N\left(\mathrm{H}_{2}\right) \\
\left(\times 10^{23} \mathrm{~cm}^{-2}\right)\end{array}$ & Env. & Comment \\
\hline$(1)$ & $(2)$ & (3) & $(4)$ & $(5)$ & $(6)$ & $(7)$ & $(8)$ & $(9)$ & $(10)$ \\
\hline G206.93-16.61East1 & $05: 41: 40.54$ & $-02: 17: 04.3$ & starless & 0.42 & 7 & $16.8 \pm 5.3$ & $4.1 \pm 1.5$ & OB & PGCC G206.93-16.61, NGC2023, Orion B \\
\hline G206.93-16.61East2 & $05: 41: 37.32$ & $-02: 17: 16.3$ & protostellar & 0.42 & 7 & $16.8 \pm 5.3$ & $4.5 \pm 1.5$ & OB & HOPS 298, PGCC G206.93-16.61, NGC2023, Orion B \\
\hline G206.93-16.61West1 & $05: 41: 25.57$ & $-02: 16: 04.3$ & protostellar & 0.42 & 7 & $16.8 \pm 5.3$ & $2.4 \pm 0.9$ & OB & HOPS 300, PGCC G206.93-16.61, NGC2023, Orion B \\
\hline G206.93-16.61West3 & $05: 41: 25.04$ & $-02: 18: 08.1$ & protostellar & 0.42 & 7 & $16.8 \pm 5.3$ & $9.9 \pm 3.1$ & OB & HOPS 399, PGCC G206.93-16.61, NGC2023, Orion B \\
\hline G206.93-16.61West4 & $05: 41: 25.84$ & $-02: 19: 28.4$ & starless & 0.42 & 7 & $16.8 \pm 5.3$ & $2.2 \pm 0.7$ & OB & PGCC G206.93-16.61, NGC2023, Orion B \\
\hline G206.93-16.61West5 & $05: 41: 28.77$ & $-02: 20: 04.3$ & starless & 0.42 & 7 & $16.8 \pm 5.3$ & $5.7 \pm 1.8$ & OB & PGCC G206.93-16.61, NGC2023, Orion B \\
\hline G206.93-16.61West6 & $05: 41: 29.57$ & $-02: 21: 16.1$ & protostellar & 0.42 & 7 & $16.8 \pm 5.3$ & $2.0 \pm 0.6$ & OB & HOPS 398, PGCC G206.93-16.61, NGC2023, Orion B \\
\hline G207.36-19.82North1 & $05: 30: 50.94$ & $-04: 10: 35.6$ & protostellar & 0.39 & 7 & $11.9 \pm 1.4$ & $3.5 \pm 0.5$ & $\mathrm{OA}$ & VISION J05305129-0410322, PGCC G207.36-19.82, Orion A \\
\hline G207.36-19.82North2 & $05: 30: 50.67$ & $-04: 10: 15.6$ & protostellar & 0.39 & 7 & $11.9 \pm 1.4$ & $3.0 \pm 0.5$ & $\mathrm{OA}$ & VISION J05305129-0410322, PGCC G207.36-19.82, Orion A \\
\hline G207.36-19.82North3 & $05: 30: 46.40$ & $-04: 10: 27.6$ & starless & 0.39 & 7 & $11.9 \pm 1.4$ & $2.5 \pm 0.3$ & $\mathrm{OA}$ & PGCC G207.36-19.82, Orion A \\
\hline G207.36-19.82North4 & $05: 30: 44.81$ & $-04: 10: 27.6$ & starless & 0.39 & 7 & $11.9 \pm 1.4$ & $2.6 \pm 0.4$ & $\mathrm{OA}$ & PGCC G207.36-19.82, Orion A \\
\hline G207.36-19.82South & $05: 30: 46.81$ & $-04: 12: 29.4$ & starless & 0.39 & 7 & $11.9 \pm 1.4$ & $0.6 \pm 0.1$ & $\mathrm{OA}$ & PGCC G207.36-19.82, Orion A \\
\hline G207.3-19.8A2North1 & $05: 31: 03.40$ & $-04: 15: 46.0$ & starless & 0.39 & 7 & $11.9 \pm 1.4$ & $0.7 \pm 0.1$ & $\mathrm{OA}$ & PGCC G207.36-19.82, Orion A \\
\hline G207.3-19.8A2North2 & $05: 31: 02.06$ & $-04: 14: 57.0$ & starless & 0.39 & 7 & $11.9 \pm 1.4$ & $0.7 \pm 0.1$ & $\mathrm{OA}$ & PGCC G207.36-19.82, Orion A \\
\hline G207.3-19.8A2North3 & $05: 30: 59.99$ & $-04: 15: 39.0$ & starless & 0.39 & 7 & $11.9 \pm 1.4$ & $0.6 \pm 0.1$ & $\mathrm{OA}$ & PGCC G207.36-19.82, Orion A \\
\hline G207.3-19.8A2South & $05: 31: 03.27$ & $-04: 17: 00.0$ & starless & 0.39 & 7 & $11.9 \pm 1.4$ & $0.3 \pm 0.1$ & $\mathrm{OA}$ & PGCC G207.36-19.82, Orion A \\
\hline G208.68-19.20North1 & $05: 35: 23.37$ & $-05: 01: 28.7$ & protostellar & 0.39 & 7 & $19.7 \pm 3.8$ & $10.9 \pm 2.4$ & $\mathrm{OA}$ & HOPS 87, PGCC G208.68-19.20, ONC, OMC -3 , Orion A \\
\hline G208.68-19.20North2 & $05: 35: 20.45$ & $-05: 00: 53.0$ & protostellar & 0.39 & 7 & $19.7 \pm 3.8$ & $11.6 \pm 3.0$ & $\mathrm{OA}$ & HOPS 89, PGCC G208.68-19.20, ONC, OMC -3 , Orion A \\
\hline G208.68-19.20North3 & $05: 35: 18.03$ & $-05: 00: 20.6$ & protostellar & 0.39 & 7 & $19.7 \pm 3.8$ & $11.7 \pm 2.5$ & $\mathrm{OA}$ & HOPS 92, PGCC G208.68-19.20, ONC, OMC -3 , Orion A \\
\hline G208.68-19.20South & $05: 35: 26.33$ & $-05: 03: 56.7$ & protostellar & 0.39 & 7 & $19.7 \pm 3.8$ & $4.6 \pm 1.0$ & $\mathrm{OA}$ & HOPS 84, PGCC G208.68-19.20, ONC, OMC -3 , Orion A \\
\hline G208.89-20.04East & $05: 32: 48.40$ & $-05: 34: 47.1$ & protostellar & 0.39 & 7 & $12.8 \pm 4.2$ & $2.6 \pm 0.9$ & $\mathrm{OA}$ & WISE J053248.59-053451.2, PGCC G208.89-20.04, ONC, OMC-4, Orion A \\
\hline G209.05-19.73North & $05: 34: 03.96$ & $-05: 32: 42.5$ & starless & 0.39 & 7 & $15.6 \pm 8.4$ & $0.6 \pm 0.3$ & $\mathrm{OA}$ & PGCC G209.05-19.73, ONC, OMC-4, Orion A \\
\hline G209.05-19.73South & $05: 34: 03.12$ & $-05: 34: 11.0$ & starless & 0.39 & 7 & $15.6 \pm 8.4$ & $1.0 \pm 0.5$ & $\mathrm{OA}$ & PGCC G209.05-19.73, ONC, OMC-4, Orion A \\
\hline G209.29-19.65North1 & $05: 35: 00.25$ & $-05: 40: 02.4$ & starless & 0.39 & 7 & $17.3 \pm 5.2$ & $1.5 \pm 0.5$ & $\mathrm{OA}$ & PGCC G209.20-19.55, ONC, OMC-4, Orion A \\
\hline G209.29-19.65North2 & $05: 34: 57.30$ & $-05: 41: 44.4$ & protostellar & 0.39 & 7 & $17.3 \pm 5.2$ & $1.3 \pm 0.4$ & $\mathrm{OA}$ & WISE J053458.25-054149.9, PGCC G209.29-19.65, ONC, OMC-4, Orion A \\
\hline G209.29-19.65North3 & $05: 34: 54.75$ & $-05: 43: 34.4$ & protostellar & 0.39 & 7 & $17.3 \pm 5.2$ & $1.3 \pm 0.4$ & $\mathrm{OA}$ & [MGM2012] 1260, PGCC G209.29-19.65, ONC, OMC-4, Orion A \\
\hline G209.29-19.65South1 & $05: 34: 55.99$ & $-05: 46: 03.2$ & starless & 0.39 & 7 & $17.3 \pm 5.2$ & $3.1 \pm 1.0$ & $\mathrm{OA}$ & PGCC G209.29-19.65, ONC, OMC-4, Orion A \\
\hline G209.29-19.65South2 & $05: 34: 53.81$ & $-05: 46: 12.8$ & starless & 0.39 & 7 & $17.3 \pm 5.2$ & $3.8 \pm 1.3$ & $\mathrm{OA}$ & PGCC G209.29-19.65, ONC, OMC-4, Orion A \\
\hline G209.29-19.65South3 & $05: 34: 49.87$ & $-05: 46: 11.6$ & protostellar & 0.39 & 7 & $17.3 \pm 5.2$ & $1.6 \pm 0.6$ & $\mathrm{OA}$ & [MGM2012] 1221, PGCC G209.29-19.65, ONC, OMC-4, Orion A \\
\hline G209.55-19.68North1 & $05: 35: 08.76$ & $-05: 55: 50.4$ & protostellar & 0.39 & 7 & $14.1 \pm 4.0$ & $0.4 \pm 0.1$ & $\mathrm{OA}$ & HOPS 12, PGCC G209.55-19.68, OMC-5, Orion A \\
\hline G209.55-19.68North2 & $05: 35: 07.01$ & $-05: 56: 38.4$ & starless & 0.39 & 7 & $14.1 \pm 4.0$ & $1.9 \pm 0.6$ & $\mathrm{OA}$ & PGCC G209.55-19.68, OMC-5, Orion A \\
\hline G209.55-19.68North3 & $05: 35: 01.45$ & $-05: 55: 27.4$ & protostellar & 0.39 & 7 & $14.1 \pm 4.0$ & $2.3 \pm 0.8$ & $\mathrm{OA}$ & HOPS 16, PGCC G209.55-19.68, OMC-5, Orion A \\
\hline G209.55-19.68South1 & $05: 35: 13.25$ & $-05: 57: 54.4$ & protostellar & 0.39 & 7 & $14.1 \pm 4.0$ & $0.8 \pm 0.2$ & $\mathrm{OA}$ & HOPS 11, PGCC G209.55-19.68, OMC-5, Orion A \\
\hline G209.55-19.68South3 & $05: 35: 08.96$ & $-05: 58: 26.4$ & protostellar & 0.39 & 7 & $14.1 \pm 4.0$ & $1.0 \pm 0.3$ & $\mathrm{OA}$ & HOPS 10, PGCC G209.55-19.68, OMC-5, Orion A \\
\hline G209.77-19.40East1 & $05: 36: 32.45$ & $-06: 01: 16.7$ & protostellar & 0.43 & 7 & $13.9 \pm 3.4$ & $2.8 \pm 0.8$ & $\mathrm{OA}$ & HOPS 192, PGCC G209.77-19.40, L1641, Orion A \\
\hline G209.77-19.40East2 & $05: 36: 32.19$ & $-06: 02: 04.7$ & starless & 0.43 & 7 & $13.9 \pm 3.4$ & $3.9 \pm 1.1$ & $\mathrm{OA}$ & PGCC G209.77-19.40, L1641, Orion A \\
\hline G209.77-19.40East3 & $05: 36: 35.94$ & $-06: 02: 44.7$ & starless & 0.43 & 7 & $13.9 \pm 3.4$ & $3.3 \pm 0.8$ & $\mathrm{OA}$ & PGCC G209.77-19.40, L1641, Orion A \\
\hline G209.77-19.40West & $05: 36: 21.19$ & $-06: 01: 32.7$ & starless & 0.43 & 7 & $13.9 \pm 3.4$ & $1.1 \pm 0.4$ & $\mathrm{OA}$ & PGCC G209.77-19.40, L1641, Orion A \\
\hline G209.77-19.61East & $05: 35: 52.23$ & $-06: 10: 00.8$ & protostellar & 0.43 & 7 & $13.3 \pm 4.7$ & $1.5 \pm 0.6$ & $\mathrm{OA}$ & HOPS 194, PGCC G209.77-19.61, L1641, Orion A \\
\hline
\end{tabular}


Table 1 (continued)

\begin{tabular}{|c|c|c|c|c|c|c|c|c|c|}
\hline SCUBA-2 core & $\begin{array}{c}\text { R.A.(J2000) } \\
\text { (hh:mm:ss.ss) } \\
(2)\end{array}$ & $\begin{array}{l}\text { Decl.(J2000) } \\
\begin{array}{c}\text { (dd:mm:ss.s) } \\
(3)\end{array}\end{array}$ & YSO ass. & $\begin{array}{l}\text { Dist. } \\
\text { (kpc) } \\
(5)\end{array}$ & Ref & $\begin{array}{l}T_{\mathrm{d}} \\
(\mathrm{K}) \\
(7)\end{array}$ & $\begin{array}{c}N\left(\mathrm{H}_{2}\right) \\
\left(\times 10^{23} \mathrm{~cm}^{-2}\right) \\
(8)\end{array}$ & Env. & Comment \\
\hline G209.77-19.61West & $05: 35: 37.21$ & $-06: 09: 44.8$ & starless & 0.43 & 7 & $13.3 \pm 4.7$ & $1.5 \pm 0.5$ & $\mathrm{OA}$ & PGCC G209.77-19.61, L1641, Orion A \\
\hline G209.79-19.80East & $05: 35: 21.92$ & $-06: 13: 08.8$ & protostellar & 0.43 & 7 & $13.7 \pm 3.5$ & $1.0 \pm 0.3$ & $\mathrm{OA}$ & HOPS 198, PGCC G209.79-19.80, L1641, Orion A \\
\hline G209.79-19.80West & $05: 35: 11.19$ & $-06: 14: 00.7$ & starless & 0.43 & 7 & $13.7 \pm 3.5$ & $2.7 \pm 0.8$ & OA & PGCC G209.79-19.80, L1641, Orion A \\
\hline G209.94-19.52North & $05: 36: 11.55$ & $-06: 10: 44.8$ & protostellar & 0.43 & 7 & $16.3 \pm 5.9$ & $1.9 \pm 0.8$ & OA & [MGM2012] 1025, PGCC G209.94-19.52, L1641, Orion A \\
\hline G209.94-19.52South1 & $05: 36: 24.96$ & $-06: 14: 04.7$ & starless & 0.43 & 7 & $16.3 \pm 5.9$ & $1.5 \pm 0.6$ & $\mathrm{OA}$ & PGCC G209.94-19.52, L1641, Orion A \\
\hline G209.94-19.52South2 & $05: 36: 37.03$ & $-06: 15: 00.6$ & protostellar & 0.43 & 7 & $16.3 \pm 5.9$ & $1.2 \pm 0.5$ & $\mathrm{OA}$ & HOPS 185, PGCC G209.94-19.52, L1641, Orion A \\
\hline G210.37-19.53North & $05: 36: 55.03$ & $-06: 34: 33.2$ & starless & 0.43 & 7 & $14.0 \pm 4.1$ & $0.7 \pm 0.2$ & OA & PGCC G210.37-19.53, L1641, Orion A \\
\hline G210.37-19.53South & $05: 37: 00.55$ & $-06: 37: 10.2$ & protostellar & 0.43 & 7 & $14.0 \pm 4.1$ & $1.1 \pm 0.3$ & OA & HOPS 164, PGCC G210.37-19.53, L1641, Orion A \\
\hline G210.49-19.79East1 & $05: 36: 25.28$ & $-06: 44: 42.8$ & protostellar & 0.43 & 7 & $11.8 \pm 2.7$ & $5.7 \pm 1.7$ & $\mathrm{OA}$ & HOPS 166, PGCC G210.49-19.79, L1641, Orion A \\
\hline G210.49-19.79East2 & $05: 36: 23.13$ & $-06: 46: 10.8$ & protostellar & 0.43 & 7 & $11.8 \pm 2.7$ & $8.4 \pm 2.2$ & OA & HOPS 203, PGCC G210.49-19.79, L1641, Orion A \\
\hline G210.49-19.79West & $05: 36: 18.40$ & $-06: 45: 26.8$ & protostellar & 0.43 & 7 & $11.8 \pm 2.7$ & $11.3 \pm 2.9$ & OA & HOPS 168, PGCC G210.49-19.79, L1641, Orion A \\
\hline G210.82-19.47North1 & $05: 37: 56.56$ & $-06: 56: 35.1$ & protostellar & 0.43 & 7 & $16.2 \pm 7.9$ & $0.4 \pm 0.2$ & OA & HOPS 157, PGCC G210.82-19.47, L1641, Orion A \\
\hline G210.82-19.47North2 & $05: 37: 59.84$ & $-06: 57: 09.9$ & starless & 0.43 & 7 & $16.2 \pm 7.9$ & $0.4 \pm 0.2$ & $\mathrm{OA}$ & PGCC G210.82-19.47, L1641, Orion A \\
\hline G210.97-19.33North & $05: 38: 40.36$ & $-06: 58: 21.9$ & protostellar & 0.43 & 7 & $12.8 \pm 1.1$ & $0.8 \pm 0.1$ & $\mathrm{OA}$ & HOPS 149, PGCC G210.97-19.33, L1641, Orion A \\
\hline G210.97-19.33South1 & $05: 38: 49.46$ & $-07: 01: 17.9$ & protostellar & 0.43 & 7 & $12.8 \pm 1.1$ & $2.1 \pm 0.4$ & $\mathrm{OA}$ & HOPS 139, PGCC G210.97-19.33, L1641, Orion A \\
\hline G210.97-19.33South2 & $05: 38: 45.30$ & $-07: 01: 04.4$ & protostellar & 0.43 & 7 & $12.8 \pm 1.1$ & $1.2 \pm 0.3$ & OA & HOPS 377, PGCC G210.97-19.33, L1641, Orion A \\
\hline G211.01-19.54North & $05: 37: 57.23$ & $-07: 06: 56.7$ & protostellar & 0.43 & 7 & $14.7 \pm 8.4$ & $2.5 \pm 1.4$ & $\mathrm{OA}$ & HOPS 153, PGCC G211.01-19.54, L1641, Orion A \\
\hline G211.01-19.54South & $05: 37: 59.04$ & $-07: 07: 32.7$ & protostellar & 0.43 & 7 & $14.7 \pm 8.4$ & $2.5 \pm 1.4$ & OA & HOPS 152, PGCC G211.01-19.54, L1641, Orion A \\
\hline G211.16-19.33North1 & $05: 39: 11.80$ & $-07: 10: 29.9$ & protostellar & 0.43 & 7 & $12.5 \pm 1.8$ & $0.9 \pm 0.1$ & $\mathrm{OA}$ & HOPS 129, PGCC G211.16-19.33, L1641, Orion A \\
\hline G211.16-19.33North2 & $05: 39: 05.89$ & $-07: 10: 37.9$ & protostellar & 0.43 & 7 & $12.5 \pm 1.8$ & $0.8 \pm 0.1$ & OA & HOPS 133, PGCC G211.16-19.33, L1641, Orion A \\
\hline G211.16-19.33North3 & $05: 39: 02.26$ & $-07: 11: 07.9$ & starless & 0.43 & 7 & $12.5 \pm 1.8$ & $0.6 \pm 0.1$ & $\mathrm{OA}$ & PGCC G211.16-19.33, L1641, Orion A \\
\hline G211.16-19.33North4 & $05: 38: 55.67$ & $-07: 11: 25.9$ & protostellar & 0.43 & 7 & $12.5 \pm 1.8$ & $0.4 \pm 0.1$ & OA & Herschel J053854.1-071123, PGCC G211.16-19.33, L1641, Orion A \\
\hline G211.16-19.33North5 & $05: 38: 46.00$ & $-07: 10: 41.9$ & protostellar & 0.43 & 7 & $12.5 \pm 1.8$ & $0.7 \pm 0.1$ & OA & HOPS 135, PGCC G211.09-19.47, L1641, Orion A \\
\hline G211.16-19.33South & $05: 39: 02.94$ & $-07: 12: 49.9$ & protostellar & 0.43 & 7 & $12.5 \pm 1.8$ & $1.0 \pm 0.2$ & OA & HOPS 130, PGCC G211.16-19.33, L1641, Orion A \\
\hline G211.47-19.27North & $05: 39: 57.27$ & $-07: 29: 38.3$ & protostellar & 0.43 & 7 & $12.4 \pm 1.1$ & $4.1 \pm 0.4$ & $\mathrm{OA}$ & HOPS 290, PGCC G211.47-19.27, L1641, Orion A \\
\hline G211.47-19.27South & $05: 39: 55.92$ & $-07: 30: 28.3$ & protostellar & 0.43 & 7 & $12.4 \pm 1.1$ & $7.2 \pm 0.8$ & OA & HOPS 288, PGCC G211.47-19.27, L1641, Orion A \\
\hline G211.72-19.25North & $05: 40: 13.72$ & $-07: 32: 16.8$ & protostellar & 0.43 & 7 & $12.6 \pm 3.4$ & $0.9 \pm 0.3$ & OA & WISE J054013.78-073216.0, PGCC G211.72-19.25, L1641, Orion A \\
\hline G211.72-19.25South1 & $05: 40: 19.04$ & $-07: 34: 28.8$ & starless & 0.43 & 7 & $12.6 \pm 3.4$ & $0.8 \pm 0.3$ & OA & PGCC G211.72-19.25, L1641, Orion A \\
\hline G212.10-19.15North1 & $05: 41: 21.56$ & $-07: 52: 27.7$ & protostellar & 0.43 & 7 & $10.8 \pm 1.4$ & $1.9 \pm 0.3$ & $\mathrm{OA}$ & Herschel J054120.5-075237, PGCC G212.10-19.15, Orion A \\
\hline G212.10-19.15North2 & $05: 41: 23.98$ & $-07: 53: 48.5$ & protostellar & 0.43 & 7 & $10.8 \pm 1.4$ & $1.8 \pm 0.3$ & $\mathrm{OA}$ & HOPS 263, PGCC G212.10-19.15, Orion A \\
\hline G212.10-19.15North3 & $05: 41: 24.82$ & $-07: 55: 08.5$ & protostellar & 0.43 & 7 & $10.8 \pm 1.4$ & $2.0 \pm 0.3$ & OA & HOPS 254, PGCC G212.10-19.15, Orion A \\
\hline G212.10-19.15South & $05: 41: 26.39$ & $-07: 56: 51.8$ & protostellar & 0.43 & 7 & $10.8 \pm 1.4$ & $1.9 \pm 0.3$ & $\mathrm{OA}$ & HOPS 247 , PGCC G212.10-19.15, Orion A \\
\hline G212.84-19.45North & $05: 41: 32.14$ & $-08: 40: 10.9$ & protostellar & 0.43 & 7 & $11.7 \pm 1.1$ & $2.0 \pm 0.2$ & $\mathrm{OA}$ & HOPS 224 , PGCC G212.84-19.45, Orion A \\
\hline G212.84-19.45South & $05: 41: 29.70$ & $-08: 43: 00.2$ & protostellar & 0.43 & 7 & $11.7 \pm 1.1$ & $0.7 \pm 0.1$ & $\mathrm{OA}$ & HOPS 219, PGCC G212.84-19.45, Orion A \\
\hline G215.44-16.38 & $05: 56: 58.45$ & $-09: 32: 42.3$ & starless & 0.43 & 7 & $12.1 \pm 0.8$ & $0.6 \pm 0.1$ & $\mathrm{OA}$ & PGCC G215.44-16.38, Orion A \\
\hline G215.87-17.62North & $05: 53: 41.91$ & $-10: 24: 02.0$ & protostellar & 0.43 & 7 & $12.2 \pm 1.2$ & $0.6 \pm 0.1$ & $\mathrm{OA}$ & WISE J055342.54-102400.5, PGCC G215.82-17.45, Orion A \\
\hline G215.87-17.62Middle & $05: 53: 32.41$ & $-10: 25: 06.1$ & protostellar & 0.43 & 7 & $12.2 \pm 1.2$ & $0.5 \pm 0.1$ & $\mathrm{OA}$ & WISE J055332.75-102510.2, PGCC G215.87-17.62, Orion A \\
\hline G215.87-17.62South & $05: 53: 26.43$ & $-10: 27: 26.0$ & protostellar & 0.43 & 7 & $12.2 \pm 1.2$ & $0.6 \pm 0.1$ & $\mathrm{OA}$ & WISE J055325.06-102730.1, PGCC G215.87-17.62, Orion A \\
\hline SCOPEG139.12-03.23 & $02: 50: 21.84$ & $+55: 51: 36.0$ & protostellar & 2.22 & 13 & $10.6 \pm 1.3$ & $0.7 \pm 0.1$ & $\mathrm{H}$ & WISE J025022.14+555128.4, PGCC G139.19-3.29 \\
\hline
\end{tabular}

Table 1 continued 
Table 1 (continued)

\begin{tabular}{|c|c|c|c|c|c|c|c|c|c|}
\hline SCUBA-2 core & $\begin{array}{l}\text { R.A.(J2000) } \\
\text { (hh:mm:ss.ss) }\end{array}$ & $\begin{array}{l}\text { Decl.(J2000) } \\
\text { (dd:mm:ss.s) }\end{array}$ & YSO ass. & $\begin{array}{l}\text { Dist. } \\
\text { (kpc) }\end{array}$ & Ref & $\begin{array}{l}T_{\mathrm{d}} \\
(\mathrm{K})\end{array}$ & $\begin{array}{c}N\left(\mathrm{H}_{2}\right) \\
\left(\times 10^{23} \mathrm{~cm}^{-2}\right)\end{array}$ & Env. & Comment \\
\hline (1) & $(2)$ & (3) & (4) & $(5)$ & $(6)$ & $(7)$ & $(8)$ & (9) & (10) \\
\hline SCOPEG159.21-20.13 & $03: 33: 16.08$ & $+31: 06: 50.4$ & protostellar & 0.30 & 10 & $11.4 \pm 1.5$ & $0.9 \pm 0.2$ & $\mathrm{H}$ & [EES2009] Per-emb 10, PGCC G159.19-20.11, Banard 1, Perseus \\
\hline SCOPEG159.18-20.09 & $03: 33: 17.76$ & $+31: 09: 32.4$ & protostellar & 0.30 & 10 & $11.4 \pm 1.5$ & $3.0 \pm 0.7$ & $\mathrm{H}$ & SSTc2d J033317.8+310931, PGCC G159.19-20.11, Banard 1, Perseus \\
\hline SCOPEG159.22-20.11 & $03: 33: 21.36$ & $+31: 07: 26.4$ & protostellar & 0.30 & 10 & $11.4 \pm 1.5$ & $2.6 \pm 0.6$ & $\mathrm{H}$ & [SDA2014] West41, PGCC G159.19-20.11, Banard 1, Perseus \\
\hline SCOPEG162.46-08.67 & $04: 21: 38.16$ & $+37: 34: 37.2$ & protostellar & 0.45 & 8 & $12.1 \pm 0.7$ & $0.5 \pm 0.1$ & $\mathrm{H}$ & WISE J042137.96+373441.7, PGCC G162.44-8.70, California \\
\hline SCOPEG162.48-08.68 & $04: 21: 41.28$ & $+37: 33: 57.6$ & protostellar & 0.45 & 8 & $12.1 \pm 0.7$ & $0.4 \pm 0.1$ & $\mathrm{H}$ & SSTgbs J0421408+373359, PGCC G162.44-8.70, California \\
\hline SCOPEG171.50-14.91 & $04: 28: 39.36$ & $+26: 51: 32.4$ & protostellar & 0.14 & 4 & $9.2 \pm 2.1$ & $1.2 \pm 0.3$ & $\mathrm{H}$ & NAME LDN 1521F IRS, PGCC G171.49-14.91, L1521F, Taurus \\
\hline SCOPEG172.88+02.26 & $05: 36: 51.60$ & $+36: 10: 40.8$ & protostellar & 1.30 & 14 & $11.6 \pm 2.4$ & $1.1 \pm 0.3$ & $\mathrm{H}$ & WISE J053651.31+361037.1, PGCC G172.84+2.28, L1525, Auriga \\
\hline SCOPEG172.88+02.27 & $05: 36: 53.76$ & $+36: 10: 33.6$ & protostellar & 1.30 & 14 & $11.6 \pm 2.4$ & $1.1 \pm 0.4$ & $\mathrm{H}$ & WISE J053653.75+361033.5, PGCC G172.84+2.28, L1525, Auriga \\
\hline SCOPEG172.89+02.27 & $05: 36: 54.96$ & $+36: 10: 12.0$ & protostellar & 1.30 & 14 & $11.6 \pm 2.4$ & $0.7 \pm 0.2$ & $\mathrm{H}$ & WISE J053654.91+361008.2, PGCC G172.84+2.28, L1525, Auriga \\
\hline SCOPEG173.17+02.36 & $05: 38: 00.48$ & $+35: 58: 58.8$ & protostellar & 1.30 & 14 & $14.8 \pm 3.0$ & $0.6 \pm 0.1$ & $\mathrm{H}$ & WISE J053800.11+355903.7, PGCC G173.12+2.36, L1525, Auriga \\
\hline SCOPEG173.18+02.35 & $05: 38: 01.68$ & $+35: 58: 15.6$ & protostellar & 1.30 & 14 & $14.8 \pm 3.0$ & $0.7 \pm 0.2$ & $\mathrm{H}$ & Herschel J053801.5+355817, PGCC G173.12+2.36, L1525, Auriga \\
\hline SCOPEG173.19+02.35 & $05: 38: 01.68$ & $+35: 57: 39.6$ & protostellar & 1.30 & 14 & $14.8 \pm 3.0$ & $0.4 \pm 0.1$ & $\mathrm{H}$ & Herschel J053801.3+355734, PGCC G173.12+2.36, L1525, Auriga \\
\hline SCOPEG178.27-00.60 & $05: 39: 06.48$ & $+30: 05: 24.0$ & protostellar & 0.96 & 13 & $11.9 \pm 0.8$ & $0.4 \pm 0.1$ & G & Herschel J053906.4+300524, PGCC G178.28-0.58 \\
\hline SCOPEG178.28-00.60 & $05: 39: 07.44$ & $+30: 04: 44.4$ & protostellar & 0.96 & 13 & $11.9 \pm 0.8$ & $0.2 \pm 0.1$ & G & Herschel J053907.3+300452, PGCC G178.28-0.58 \\
\hline SCOPEG195.71-02.32 & $06: 10: 50.40$ & $+14: 10: 15.6$ & protostellar & 2.05 & 2 & $12.2 \pm 1.4$ & $0.7 \pm 0.1$ & $\mathrm{H}$ & [MJR2015] 1377, PGCC G195.74-2.30 \\
\hline SCOPEG195.74-02.30 & $06: 10: 58.32$ & $+14: 09: 28.8$ & protostellar & 2.05 & 2 & $12.2 \pm 1.4$ & $1.1 \pm 0.3$ & $\mathrm{H}$ & WISE J061058.71+140929.6, PGCC G195.74-2.30 \\
\hline SCOPEG202.30+02.53 & $06: 40: 58.32$ & $+10: 36: 54.0$ & protostellar & 0.76 & 17 & $14.5 \pm 1.7$ & $1.3 \pm 0.2$ & $\mathrm{H}$ & 2MASS J06405810+1036525, PGCC G202.36+2.50, NGC2264 \\
\hline SCOPEG202.31+02.52 & $06: 40: 59.28$ & $+10: 36: 07.2$ & protostellar & 0.76 & 17 & $14.5 \pm 1.7$ & $0.7 \pm 0.1$ & $\mathrm{H}$ & [RPG2014] 61848, PGCC G202.36+2.50, NGC2264 \\
\hline SCOPEG202.32+02.53 & $06: 41: 01.92$ & $+10: 35: 24.0$ & starless & 0.76 & 17 & $14.5 \pm 1.7$ & $0.4 \pm 0.1$ & $\mathrm{H}$ & PGCC G202.36+2.50, NGC2264 \\
\hline SCOPEG006.01+36.74 & $15: 54: 08.64$ & $-02: 52: 44.4$ & starless & 0.11 & 16 & $11.2 \pm 0.4$ & $0.3 \pm 0.1$ & $\mathrm{H}$ & PGCC G6.04+36.77, L183 \\
\hline SCOPEG001.37+20.95 & $16: 34: 35.28$ & $-15: 46: 55.2$ & starless & 0.12 & 9 & $12.1 \pm 0.5$ & $0.6 \pm 0.1$ & $\mathrm{H}$ & PGCC G1.40+20.93, L43, Ophiuchus \\
\hline SCOPEG010.19+02.41 & $17: 59: 15.36$ & $-18: 56: 42.0$ & protostellar & 0.81 & 18 & $15.0 \pm 1.5$ & $0.2 \pm 0.1$ & $\mathrm{H}$ & [MJR2015] 115, PGCC G10.20+2.40 \\
\hline SCOPEG005.91-00.95 & $18: 02: 42.00$ & $-24: 19: 04.8$ & protostellar & 2.25 & 1 & $16.4 \pm 7.6$ & $0.6 \pm 0.3$ & G & SSTGLMC G005.9126-00.9525, PGCC G6.87-0.39, NGC6530 \\
\hline SCOPEG005.88-01.01 & $18: 02: 50.16$ & $-24: 22: 26.4$ & protostellar & 2.25 & 1 & $17.6 \pm 5.2$ & $0.4 \pm 0.1$ & G & MIRES G005.8802-01.0041, PGCC G4.81-1.42, NGC6530 \\
\hline SCOPEG005.92-00.99 & $18: 02: 51.36$ & $-24: 20: 06.0$ & protostellar & 2.25 & 1 & $16.4 \pm 7.6$ & $0.5 \pm 0.3$ & G & Herschel G005.9164-0.9913, PGCC G6.87-0.39, NGC6530 \\
\hline SCOPEG005.90-01.01 & $18: 02: 53.04$ & $-24: 21: 43.2$ & protostellar & 2.25 & 1 & $16.4 \pm 7.6$ & $0.4 \pm 0.2$ & G & WISE J180253.28-242153.8, PGCC G6.87-0.39, NGC6530 \\
\hline SCOPEG005.91-01.02 & $18: 02: 58.08$ & $-24: 21: 25.2$ & protostellar & 2.25 & 1 & $16.4 \pm 7.6$ & $0.5 \pm 0.2$ & $\mathrm{G}$ & MIRES G005.9108-01.0229, PGCC G6.87-0.39, NGC6530 \\
\hline SCOPEG017.38+02.26 & $18: 14: 18.96$ & $-12: 43: 58.8$ & protostellar & 2.56 & 12 & $15.3 \pm 2.7$ & $0.3 \pm 0.1$ & $\mathrm{H}$ & WISE J181418.81-124358.7, PGCC G17.37+2.26 \\
\hline SCOPEG017.38+02.25 & $18: 14: 21.12$ & $-12: 44: 38.4$ & protostellar & 2.56 & 12 & $15.3 \pm 2.7$ & $0.6 \pm 0.1$ & $\mathrm{H}$ & MSX6C G017.3765+02.2512, PGCC G17.37+2.26 \\
\hline SCOPEG017.37+02.24 & $18: 14: 22.56$ & $-12: 45: 25.2$ & protostellar & 2.56 & 12 & $15.3 \pm 2.7$ & $0.4 \pm 0.1$ & $\mathrm{H}$ & WISE J181422.18-124521.2, PGCC G17.37+2.26 \\
\hline SCOPEG017.36+02.23 & $18: 14: 24.00$ & $-12: 45: 54.0$ & protostellar & 2.56 & 12 & $15.3 \pm 2.7$ & $0.6 \pm 0.1$ & $\mathrm{H}$ & WISE J181424.00-124548.6, PGCC G17.37+2.26 \\
\hline SCOPEG014.20-00.18 & $18: 16: 55.44$ & $-16: 41: 45.6$ & protostellar & 3.07 & 13 & $15.8 \pm 4.3$ & $0.6 \pm 0.2$ & G & WISE J181656.04-164141.5, PGCC G14.21-0.19 \\
\hline SCOPEG014.23-00.17 & $18: 16: 58.80$ & $-16: 39: 50.4$ & starless & 3.06 & 13 & $15.8 \pm 4.3$ & $0.5 \pm 0.1$ & G & PGCC G14.21-0.19 \\
\hline SCOPEG014.18-00.23 & $18: 17: 05.28$ & $-16: 43: 44.4$ & protostellar & 3.15 & 13 & $15.8 \pm 4.3$ & $0.5 \pm 0.2$ & G & Herschel G014.1897-0.2273, PGCC G14.21-0.19 \\
\hline SCOPEG014.71-00.15 & $18: 17: 50.88$ & $-16: 13: 48.0$ & protostellar & 3.20 & 13 & $14.4 \pm 8.6$ & $0.7 \pm 0.5$ & G & MIRES G014.7115-00.1518, PGCC G14.71-0.19 \\
\hline SCOPEG014.72-00.20 & $18: 18: 03.60$ & $-16: 14: 42.0$ & protostellar & 3.06 & 13 & $14.4 \pm 8.6$ & $0.6 \pm 0.4$ & G & MIRES G014.7236-00.2003, PGCC G14.71-0.19 \\
\hline SCOPEG014.69-00.22 & $18: 18: 03.60$ & $-16: 17: 09.6$ & protostellar & 3.06 & 13 & $14.4 \pm 8.6$ & $0.7 \pm 0.4$ & $\mathrm{G}$ & MIRES G014.6882-00.2217, PGCC G14.71-0.19 \\
\hline SCOPEG014.71-00.22 & $18: 18: 06.24$ & $-16: 15: 54.0$ & protostellar & 3.04 & 13 & $14.4 \pm 8.6$ & $0.5 \pm 0.3$ & G & Herschel G014.7126-0.2269, PGCC G14.71-0.19 \\
\hline SCOPEG014.11-00.57 & $18: 18: 13.20$ & $-16: 57: 21.6$ & protostellar & 1.85 & 13 & $15.7 \pm 3.7$ & $2.2 \pm 0.9$ & G & Herschel G014.1139-0.5745, PGCC G14.14-0.55 \\
\hline
\end{tabular}


Table 1 (continued)

\begin{tabular}{|c|c|c|c|c|c|c|c|c|c|}
\hline SCUBA-2 core & $\begin{array}{l}\text { R.A.(J2000) } \\
\text { (hh:mm:ss.ss) }\end{array}$ & $\begin{array}{l}\text { Decl.(J2000) } \\
\text { (dd:mm:ss.s) }\end{array}$ & YSO ass. & $\begin{array}{l}\text { Dist. } \\
\text { (kpc) }\end{array}$ & Ref & $\begin{array}{l}T_{\mathrm{d}} \\
(\mathrm{K})\end{array}$ & $\begin{array}{c}N\left(\mathrm{H}_{2}\right) \\
\left(\times 10^{23} \mathrm{~cm}^{-2}\right)\end{array}$ & Env. & Comment \\
\hline$(1)$ & $(2)$ & $(3)$ & $(4)$ & $(5)$ & $(6)$ & $(7)$ & $(8)$ & $(9)$ & $(10)$ \\
\hline SCOPEG014.23-00.51 & $18: 18: 12.72$ & $-16: 49: 33.6$ & protostellar & 1.85 & 13 & $15.7 \pm 3.7$ & $3.1 \pm 0.7$ & G & GAL 014.23-00.51, PGCC G14.14-0.55 \\
\hline SCOPEG016.93+00.28 & $18: 20: 35.76$ & $-14: 04: 15.6$ & protostellar & 1.87 & 13 & $13.3 \pm 5.9$ & $0.5 \pm 0.2$ & G & MSX6C G016.9261+00.2854, PGCC G16.96+0.27, NGC 6611 \\
\hline SCOPEG016.93+00.27 & $18: 20: 39.84$ & $-14: 04: 51.6$ & protostellar & 1.87 & 13 & $13.3 \pm 5.9$ & $0.5 \pm 0.3$ & G & Herschel G016.9253+0.2662, PGCC G16.96+0.27, NGC 6611 \\
\hline SCOPEG016.93+00.25 & $18: 20: 43.20$ & $-14: 05: 16.8$ & starless & 1.87 & 13 & $13.3 \pm 5.9$ & $0.5 \pm 0.3$ & $\mathrm{G}$ & PGCC G16.96+0.27, NGC 6611 \\
\hline SCOPEG016.93+00.24 & $18: 20: 44.88$ & $-14: 05: 31.2$ & starless & 1.88 & 13 & $13.3 \pm 5.9$ & $0.6 \pm 0.3$ & G & PGCC G16.96+0.27, NGC 6611 \\
\hline SCOPEG016.92+00.23 & $18: 20: 46.56$ & $-14: 06: 14.4$ & starless & 1.87 & 13 & $13.3 \pm 5.9$ & $0.5 \pm 0.2$ & $\mathrm{G}$ & PGCC G16.96+0.27, NGC 6611 \\
\hline SCOPEG016.93+00.22 & $18: 20: 50.64$ & $-14: 06: 00.0$ & protostellar & 1.87 & 13 & $13.3 \pm 5.9$ & $0.8 \pm 0.4$ & $\mathrm{G}$ & Herschel G016.9288+0.2180, PGCC G16.96+0.27, NGC 6611 \\
\hline SCOPEG016.30-00.53 & $18: 22: 20.16$ & $-15: 00: 14.4$ & starless & 3.15 & 13 & $19.6 \pm 6.1$ & $0.3 \pm 0.1$ & G & PGCC G16.36-0.62 \\
\hline SCOPEG016.34-00.59 & $18: 22: 37.20$ & $-15: 00: 00.0$ & protostellar & 3.35 & 13 & $19.6 \pm 6.1$ & $0.6 \pm 0.2$ & G & WISE J182236.21-145956.5, PGCC G16.36-0.62 \\
\hline SCOPEG016.38-00.61 & $18: 22: 47.52$ & $-14: 58: 37.2$ & protostellar & 3.36 & 13 & $19.6 \pm 6.1$ & $0.3 \pm 0.1$ & $\mathrm{G}$ & Herschel J182247.7-145848, PGCC G16.36-0.62 \\
\hline SCOPEG016.42-00.64 & $18: 22: 58.08$ & $-14: 57: 00.0$ & starless & 3.34 & 13 & $19.8 \pm 6.3$ & $0.4 \pm 0.1$ & $\mathrm{G}$ & PGCC G16.42-0.63 \\
\hline SCOPEG017.22-01.46 & $18: 27: 30.48$ & $-14: 37: 51.6$ & protostellar & 3.02 & 13 & $9.9 \pm 1.4$ & $3.9 \pm 1.1$ & G & WISE J182730.01-143747.4, PGCC G17.21-1.46 \\
\hline SCOPEG017.21-01.47 & $18: 27: 31.44$ & $-14: 38: 34.8$ & protostellar & 3.01 & 13 & $9.9 \pm 1.4$ & $2.5 \pm 0.6$ & G & WISE J182731.41-143844.9, PGCC G17.21-1.46 \\
\hline SCOPEG023.63+00.59 & $18: 32: 14.88$ & $-08: 00: 10.8$ & starless & 5.80 & 13 & $22.4 \pm 5.9$ & $0.2 \pm 0.1$ & G & PGCC G23.68+0.57, L463 \\
\hline SCOPEG023.69+00.59 & $18: 32: 21.36$ & $-07: 56: 38.4$ & protostellar & 5.82 & 13 & $22.4 \pm 5.9$ & $0.6 \pm 0.3$ & G & Herschel G023.6917+0.5888, PGCC G23.68+0.57, L463 \\
\hline SCOPEG024.02+00.24 & $18: 34: 13.44$ & $-07: 48: 32.4$ & starless & 9.21 & 13 & $20.2 \pm 7.0$ & $0.3 \pm 0.1$ & G & PGCC G24.04+0.26 \\
\hline SCOPEG024.02+00.21 & $18: 34: 18.72$ & $-07: 49: 44.4$ & starless & 6.00 & 13 & $20.2 \pm 7.0$ & $0.4 \pm 0.1$ & G & PGCC G24.04+0.26 \\
\hline SCOPEG023.32-00.29 & $18: 34: 50.16$ & $-08: 40: 51.6$ & protostellar & 5.90 & 13 & $16.9 \pm 6.6$ & $1.0 \pm 0.4$ & G & SSTGLMC G023.3227-00.2937, PGCC G23.35-0.26 \\
\hline SCOPEG026.50+00.71 & $18: 37: 07.44$ & $-05: 23: 56.4$ & protostellar & 3.02 & 13 & $15.7 \pm 4.4$ & $1.2 \pm 0.6$ & G & MSX6C G026.4958+00.7105, PGCC G26.53+0.71 \\
\hline SCOPEG037.92+02.14 & $18: 52: 53.76$ & $+05: 25: 08.4$ & protostellar & 1.90 & 13 & $14.0 \pm 4.0$ & $0.3 \pm 0.1$ & $\mathrm{H}$ & [MJR2015] 2928, PGCC G37.91+2.18 \\
\hline SCOPEG033.74-00.01 & $18: 52: 57.12$ & $+00: 43: 01.2$ & protostellar & 6.50 & 13 & $16.8 \pm 3.5$ & $0.6 \pm 0.3$ & G & Herschel J185256.6+004316, PGCC G33.72-0.02 \\
\hline SCOPEG039.74+01.99 & $18: 56: 46.56$ & $+06: 57: 39.6$ & protostellar & 1.91 & 13 & $13.6 \pm 4.7$ & $0.4 \pm 0.1$ & G & [MJR2015] 2954, PGCC G39.73+1.91 \\
\hline SCOPEG035.48-00.29 & $18: 57: 06.96$ & $+02: 08: 24.0$ & protostellar & 2.21 & 13 & $14.0 \pm 5.7$ & $0.6 \pm 0.4$ & G & SSTGLMC G035.4858-00.2876, PGCC G35.49-0.31 \\
\hline SCOPEG035.52-00.27 & $18: 57: 08.40$ & $+02: 10: 48.0$ & protostellar & 2.25 & 13 & $14.0 \pm 5.7$ & $0.7 \pm 0.3$ & G & Herschel G035.5235-0.2728, PGCC G35.49-0.31 \\
\hline SCOPEG035.48-00.31 & $18: 57: 11.28$ & $+02: 07: 30.0$ & protostellar & 2.23 & 13 & $14.0 \pm 5.7$ & $0.6 \pm 0.4$ & G & MIREX G035.4823-00.3086, PGCC G35.49-0.31 \\
\hline SCOPEG034.75-01.38 & $18: 59: 41.04$ & $+00: 59: 06.0$ & protostellar & 2.19 & 13 & $14.5 \pm 4.4$ & $1.2 \pm 0.4$ & G & Herschel J185941.2+005908, PGCC G34.73-1.39 \\
\hline SCOPEG035.36-01.77 & $19: 02: 11.04$ & $+01: 21: 00.0$ & protostellar & 2.21 & 13 & $13.6 \pm 6.8$ & $1.0 \pm 0.6$ & G & Herschel J190210.6+012102, PGCC G35.52-1.46, W48 \\
\hline SCOPEG035.36-01.78 & $19: 02: 12.72$ & $+01: 20: 52.8$ & starless & 2.21 & 13 & $13.6 \pm 6.8$ & $0.7 \pm 0.4$ & G & PGCC G35.52-1.46, W48 \\
\hline SCOPEG035.35-01.80 & $19: 02: 16.08$ & $+01: 19: 48.0$ & protostellar & 2.21 & 13 & $13.6 \pm 6.8$ & $0.4 \pm 0.2$ & G & WISE J190216.70+011946.7, PGCC G35.52-1.46, W48 \\
\hline SCOPEG057.11+03.66 & $19: 23: 49.20$ & $+23: 07: 58.8$ & starless & 0.80 & 13 & $11.7 \pm 0.6$ & $0.3 \pm 0.1$ & $\mathrm{H}$ & PGCC G57.11+3.65, L769 \\
\hline SCOPEG057.10+03.63 & $19: 23: 56.88$ & $+23: 06: 28.8$ & protostellar & 0.80 & 13 & $11.7 \pm 0.6$ & $0.5 \pm 0.1$ & $\mathrm{H}$ & WISE J192356.78+230633.0, PGCC G57.11+3.65, L769 \\
\hline SCOPEG069.80-01.67 & $20: 13: 32.40$ & $+31: 21: 50.4$ & protostellar & 2.48 & 13 & $14.3 \pm 1.8$ & $0.3 \pm 0.1$ & G & [MJR2015] 3117, PGCC G69.82-1.65 \\
\hline SCOPEG069.81-01.67 & $20: 13: 33.84$ & $+31: 22: 01.2$ & protostellar & 2.48 & 13 & $14.3 \pm 1.8$ & $0.4 \pm 0.1$ & G & WISE J201333.64+312206.3, PGCC G69.82-1.65 \\
\hline SCOPEG070.40-01.39 & $20: 14: 01.20$ & $+32: 00: 50.4$ & protostellar & 2.48 & 13 & $12.1 \pm 1.4$ & $0.4 \pm 0.1$ & G & [MJR2015] 3182, PGCC G70.34-1.40 \\
\hline SCOPEG074.10+00.11 & $20: 17: 56.40$ & $+35: 55: 22.8$ & protostellar & 4.34 & 13 & $12.4 \pm 1.8$ & $0.7 \pm 0.2$ & $\mathrm{G}$ & Herschel J201756.3+355525, PGCC G74.12+0.15 \\
\hline SCOPEG074.11+00.11 & $20: 17: 58.56$ & $+35: 55: 51.6$ & protostellar & 4.26 & 13 & $12.4 \pm 1.8$ & $0.6 \pm 0.1$ & G & WISE J201758.56+355552.3, PGCC G74.12+0.15 \\
\hline SCOPEG082.36-01.83 & $20: 51: 16.56$ & $+41: 22: 58.8$ & starless & 1.54 & 13 & $11.4 \pm 2.9$ & $0.4 \pm 0.1$ & $\mathrm{G}$ & PGCC G82.39-1.84 \\
\hline SCOPEG082.40-01.84 & $20: 51: 24.96$ & $+41: 24: 46.8$ & protostellar & 1.52 & 13 & $11.4 \pm 2.9$ & $0.4 \pm 0.2$ & G & [MJR2015] 3335, PGCC G82.39-1.84 \\
\hline SCOPEG082.41-01.84 & $20: 51: 27.36$ & $+41: 25: 22.8$ & starless & 1.53 & 13 & $11.4 \pm 2.9$ & $0.4 \pm 0.1$ & G & PGCC G82.39-1.84 \\
\hline
\end{tabular}

Table 1 continued 
Table 1 (continued)

\begin{tabular}{|c|c|c|c|c|c|c|c|c|c|}
\hline SCUBA-2 core & $\begin{array}{c}\text { R.A.(J2000) } \\
\text { (hh:mm:ss.ss) } \\
(2)\end{array}$ & $\begin{array}{c}\text { Decl.(J2000) } \\
\text { (dd:mm:ss.s) } \\
(3)\end{array}$ & YSO ass. & $\begin{array}{l}\text { Dist. } \\
\text { (kpc) } \\
(5)\end{array}$ & Ref & $\begin{array}{l}T_{\mathrm{d}} \\
(\mathrm{K}) \\
(7)\end{array}$ & $\begin{array}{c}N\left(\mathrm{H}_{2}\right) \\
\left(\times 10^{23} \mathrm{~cm}^{-2}\right) \\
(8)\end{array}$ & Env. & Comment \\
\hline SCOPEG082.42-01.84 & $20: 51: 28.80$ & $+41: 25: 48.0$ & starless & 1.52 & 13 & $1.4 \pm 2.9$ & $0.4 \pm 0.1$ & G & PGCC G82.39-1.84 \\
\hline SCOPEG091.86+04.17 & $21: 00: 24.96$ & $+52: 30: 18.0$ & protostellar & 0.80 & 6 & $12.2 \pm 2.2$ & $0.6 \pm 0.2$ & $\mathrm{H}$ & WISE J210025.23+523016.9, PGCC G91.87+4.18, L1004, Cygnus \\
\hline SCOPEG091.85+04.12 & $21: 00: 38.40$ & $+52: 27: 57.6$ & protostellar & 0.80 & 6 & $12.2 \pm 2.2$ & $0.7 \pm 0.1$ & $\mathrm{H}$ & WISE J210038.77+522757.5, PGCC G91.87+4.18, L1004, Cygnus \\
\hline SCOPEG092.03+03.93 & $21: 02: 23.52$ & $+52: 28: 33.6$ & starless & 0.80 & 6 & $11.6 \pm 1.9$ & $0.3 \pm 0.1$ & $\mathrm{H}$ & PGCC G92.03+3.92, L1004, Cygnus \\
\hline SCOPEG092.27+03.79 & $21: 04: 04.56$ & $+52: 33: 43.2$ & protostellar & 0.80 & 6 & $12.3 \pm 1.3$ & $1.7 \pm 0.3$ & $\mathrm{H}$ & [MJR2015] 3554, PGCC G92.24+3.84, L1004, Cygnus \\
\hline SCOPEG087.06-04.19 & $21: 17: 43.92$ & $+43: 18: 46.8$ & protostellar & 0.67 & 13 & $14.5 \pm 1.0$ & $0.2 \pm 0.1$ & $\mathrm{H}$ & WISE J211744.02+431847.7, PGCC G87.06-4.19, L944 \\
\hline SCOPEG089.64-06.62 & $21: 37: 10.56$ & $+43: 20: 45.6$ & protostellar & 0.67 & 13 & $11.1 \pm 1.0$ & $0.7 \pm 0.2$ & $\mathrm{H}$ & [MJR2015] 3461, PGCC G89.66-6.61, L973 \\
\hline SCOPEG105.37+09.84 & $21: 43: 00.72$ & $+66: 03: 21.6$ & protostellar & 1.15 & 15 & $13.0 \pm 3.0$ & $3.7 \pm 1.0$ & $\mathrm{H}$ & [SS2009] NGC 7129-S3-U419, PGCC G105.51+9.99, L1181, NGC7129 \\
\hline SCOPEG105.41+09.88 & $21: 43: 05.28$ & $+66: 06: 54.0$ & protostellar & 1.15 & 15 & $13.0 \pm 3.0$ & $3.2 \pm 1.0$ & $\mathrm{H}$ & 2MASS J21430502+6606533, PGCC G105.51+9.99, L1181, NGC7129 \\
\hline SCOPEG093.53-04.26 & $21: 44: 52.08$ & $+47: 40: 30.0$ & protostellar & 0.49 & 3 & $11.0 \pm 1.2$ & $1.0 \pm 0.3$ & $\mathrm{H}$ & HHL 73 IRS 1, PGCC G93.54-4.28, L1035, IC5146 \\
\hline SCOPEG093.54-04.28 & $21: 44: 57.60$ & $+47: 39: 57.6$ & starless & 0.49 & 3 & $11.0 \pm 1.2$ & $0.4 \pm 0.1$ & $\mathrm{H}$ & PGCC G93.54-4.28, L1035, IC5146 \\
\hline SCOPEG107.16+05.45 & $22: 21: 18.00$ & $+63: 37: 33.6$ & protostellar & 0.76 & 5 & $15.8 \pm 3.0$ & $0.2 \pm 0.1$ & $\mathrm{H}$ & [MJR2015] 3797, PGCC G107.17+5.44, L1204, Cepheus \\
\hline SCOPEG107.30+05.64 & $22: 21: 26.16$ & $+63: 51: 28.8$ & protostellar & 0.76 & 5 & $12.9 \pm 1.4$ & $4.0 \pm 0.7$ & $\mathrm{H}$ & [SPE2008b] IRAS 22198+6336 VLA 2, PGCC G107.25+5.72, L1204, Cepheus \\
\hline SCOPEG107.18+05.43 & $22: 21: 33.60$ & $+63: 37: 19.2$ & protostellar & 0.76 & 5 & $15.8 \pm 3.0$ & $0.6 \pm 0.2$ & $\mathrm{H}$ & [HLB98] Onsala 164, PGCC G107.17+5.44, L1204, Cepheus \\
\hline SCOPEG109.81+02.70 & $22: 53: 40.32$ & $+62: 31: 55.2$ & protostellar & 0.78 & 13 & $11.7 \pm 3.1$ & $0.7 \pm 0.2$ & $\mathrm{H}$ & IRAS $22517+6215$, PGCC G109.79+2.71 \\
\hline
\end{tabular}

NoтE-Column 1: SCUBA-2 core name, Column 2-3: Coordinate in Equatorial system (J2000), Column 4: Young Stellar Object (YSO) association inferred by visual inspection with YSO information of Simbad data and protostar catalogs of WISE, Spitzer, Herschel, and GAIA, Column 5: Distance from us, Column 6: Reference for the adopted distance: 1. Aidelman et al. (2018), 2. Camargo et al. (2012), 3. Fischera \& Martin (2012), 4. Galli et al. (2018), 5. Hirota et al. (2008), 6. Humphreys (1978), 7. Kounkel et al. (2017), 8. Lada et al. (2009), 9. Lombardi et al. (2008), 10. Ortiz-Leon et al. (2018), 11. Perryman et al. (1997), 12. Pidopryhora et al. (2015), 13. Reid et al. (2016), 14. Straizys et al. (2010), 15. Straižys et al. (2014), 16. Straiżys et al. (2018), 17. Sunada et al. (2007), 18. Wang et al. (2016), Column 7: Dust temperature is from that of PGCC, Column 8: $\mathrm{H}_{2}$ column density is derived from the $850 \mu \mathrm{m}$ peak intensity of the core and the dust temperature of PGCC (Yi et al. 2018; Eden et al. 2019), Column 9: Description of environment surrounding a SCUBA-2 core: 'OL', 'OA', and 'OB' indicate the sub-regions $\lambda$ Orionis, Orion A, and Orion B of the Orion region, respectively, 'G' indicates the Galactic plane $\left(|b|<2^{\circ}\right)$, 'H' indicates high latitudes $\left(|b| \geq 2^{\circ}\right)$, Column 10: Source name of YSO, PGCC, or parent cloud. Columns 1-3 and 7-8 are from Yi et al. (2018) and Eden et al. (2019). 
Table 3. Properties of $82 \mathrm{GHz} \mathrm{CCS}, 94 \mathrm{GHz} \mathrm{CCS}, \mathrm{HC}_{3} \mathrm{~N}$, and $\mathrm{N}_{2} \mathrm{H}^{+}$lines

\begin{tabular}{|c|c|c|c|c|c|c|c|c|c|c|c|c|c|c|c|c|c|}
\hline \multirow{4}{*}{ SCUBA-2 core } & \multicolumn{3}{|c|}{$82 \mathrm{GHz} \mathrm{CCS}$} & \multicolumn{3}{|c|}{$94 \mathrm{GHz} \mathrm{CCS}$} & \multicolumn{3}{|c|}{$\mathrm{HC}_{3} \mathrm{~N}$} & \multicolumn{8}{|c|}{$\mathrm{N}_{2} \mathrm{H}^{+}$} \\
\hline & \multirow{2}{*}{$\begin{array}{c}T_{\text {peak }} \\
(\mathrm{K})\end{array}$} & $V_{\mathrm{LSR}}$ & $\Delta v$ & \multirow{2}{*}{$\begin{array}{c}T_{\text {peak }} \\
(\mathrm{K})\end{array}$} & $V_{\mathrm{LSR}}$ & $\Delta v$ & \multirow{3}{*}{$\begin{array}{l}T_{\text {peak }} \\
(\mathrm{K}) \\
(8)\end{array}$} & $V_{\mathrm{LSR}}$ & $\Delta v$ & \multirow{3}{*}{$\begin{array}{l}T_{\text {peak }} \\
(\mathrm{K}) \\
(11)\end{array}$} & \multirow{3}{*}{$\begin{array}{c}V_{\mathrm{LSR}, \mathrm{GA}} \\
\\
(12)\end{array}$} & \multirow{3}{*}{$\begin{array}{r}\Delta v_{\mathrm{GA}} \\
(\mathrm{km} \\
(13)\end{array}$} & \multirow{3}{*}{$\begin{array}{l}V_{\mathrm{LSR}, \mathrm{HFS}} \\
\left.\mathrm{s}^{-1}\right) \\
\quad(14)\end{array}$} & \multirow{3}{*}{$\begin{array}{c}\Delta v_{\mathrm{HFS}} \\
(15) \\
\end{array}$} & \multirow{3}{*}{$\begin{array}{l}T_{\mathrm{ex}} \\
(\mathrm{K}) \\
(16)\end{array}$} & \multirow{3}{*}{$\begin{array}{c}\tau \\
(17)\end{array}$} & \multirow{3}{*}{$\begin{array}{l}T_{\text {ant }} \tau \\
(\mathrm{K}) \\
(18)\end{array}$} \\
\hline & & \multicolumn{2}{|c|}{$\left(\mathrm{km} \mathrm{s}^{-1}\right)$} & & \multicolumn{2}{|c|}{$\left(\mathrm{km} \mathrm{s}^{-1}\right)$} & & \multicolumn{2}{|c|}{$\left(\mathrm{km} \mathrm{s}^{-1}\right)$} & & & & & & & & \\
\hline & $(2)$ & $(3)$ & (4) & $(5)$ & (6) & $(7)$ & & (9) & $(10)$ & & & & & & & & \\
\hline G190.15-13.75North & $\leq 0.31$ & $\cdots$ & $\cdots$ & $\leq 0.29$ & $\cdots$ & $\cdots$ & $\leq 0.27$ & $\cdots$ & $\cdots$ & $\leq 0.29$ & .. & $\cdots$ & $\cdots$ & .. & $\cdots$ & $\cdots$ & $\cdots$ \\
\hline G190.15-13.75South & $\leq 0.30$ & $\cdots$ & $\cdots$ & $\leq 0.29$ & $\cdots$ & $\cdots$ & $\leq 0.28$ & $\cdots$ & $\cdots$ & 0.51 & 1.34 & 0.19 & 1.35 & 0.30 & $5.4 \pm 2.7^{\dagger}$ & $\cdots$ & $2.8 \pm 0.4$ \\
\hline G191.90-11.21North & $\leq 0.29$ & $\cdots$ & $\cdots$ & 0.37 & 5.62 & 0.24 & $\leq 0.25$ & $\cdots$ & $\cdots$ & $\leq 0.26$ & $\cdots$ & $\cdots$ & $\cdots$ & $\cdots$ & $\ldots$ & $\cdots$ & $\ldots$ \\
\hline G191.90-11.21South & $\leq 0.26$ & $\cdots$ & .. & $\leq 0.24$ & $\cdots$ & $\cdots$ & $\leq 0.23$ & $\cdots$ & $\cdots$ & 0.72 & 10.53 & 0.38 & 10.50 & 0.40 & $4.2 \pm 0.3$ & $7.6 \pm 2.8$ & $10.9 \pm 2.1$ \\
\hline G192.12-10.90North & 0.40 & 10.17 & 0.34 & 0.42 & 10.07 & 0.15 & 0.56 & 10.08 & 0.49 & 0.94 & 10.04 & 0.33 & 10.00 & 0.41 & $7.0 \pm 0.7$ & $1.8 \pm 1.8$ & $7.6 \pm 1.3$ \\
\hline G192.12-10.90South & 0.30 & 10.28 & 0.10 & $\leq 0.27$ & $\cdots$ & $\cdots$ & $\leq 0.21$ & $\cdots$ & $\cdots$ & $\leq 0.26$ & $\cdots$ & $\cdots$ & $\cdots$ & $\cdots$ & $\cdots$ & $\cdots$ & $\cdots$ \\
\hline G192.12-11.10 & $\leq 0.24$ & $\ldots$ & $\ldots$ & $\leq 0.24$ & $\cdots$ & $\cdots$ & $\leq 0.22$ & $\cdots$ & $\cdots$ & 0.52 & 10.02 & 0.35 & 10.10 & 0.73 & $4.2 \pm 0.3$ & $3.0 \pm 1.8$ & $4.5 \pm 0.8$ \\
\hline G192.32-11.88North & $\leq 0.29$ & $\cdots$ & $\cdots$ & $\leq 0.24$ & $\cdots$ & $\cdots$ & $\leq 0.25$ & $\cdots$ & $\cdots$ & 0.85 & 12.12 & 0.59 & 12.20 & 0.66 & $4.3 \pm 0.2$ & $7.3 \pm 1.8$ & $11.2 \pm 1.6$ \\
\hline G192.32-11.88South & $\leq 0.29$ & $\ldots$ & $\ldots$ & $\leq 0.24$ & $\ldots$ & $\ldots$ & 0.39 & 12.18 & 0.64 & 1.06 & 12.08 & 0.58 & 12.10 & 0.54 & $5.0 \pm 0.2$ & $6.0 \pm 1.4$ & $13.4 \pm 1.4$ \\
\hline G196.92-10.37 & $\leq 0.32$ & $\cdots$ & $\cdots$ & $\leq 0.28$ & $\cdots$ & $\cdots$ & 0.66 & 11.83 & 1.00 & 1.07 & 11.68 & 1.02 & 11.70 & 0.84 & $6.0 \pm 0.4$ & $3.0 \pm 1.0$ & $10.0 \pm 1.2$ \\
\hline G198.69-09.12North1 & $\leq 0.25$ & $\cdots$ & $\ldots$ & $\leq 0.23$ & $\cdots$ & $\ldots$ & $\leq 0.24$ & $\ldots$ & $\ldots$ & 0.29 & 11.18 & 0.62 & 11.10 & 0.44 & $5.2 \pm 0.8$ & $1.2 \pm 3.3$ & $2.9 \pm 0.9$ \\
\hline G198.69-09.12North2 & $\leq 0.25$ & $\cdots$ & $\cdots$ & $\leq 0.25$ & $\cdots$ & $\cdots$ & 0.42 & 10.68 & 0.43 & 0.44 & 10.76 & 0.68 & 10.70 & 0.51 & $4.6 \pm 0.5$ & $2.3 \pm 2.9$ & $4.2 \pm 1.1$ \\
\hline G198.69-09.12South & $\leq 0.24$ & $\ldots$ & $\ldots$ & $\leq 0.25$ & $\ldots$ & $\ldots$ & 0.49 & 11.03 & 0.93 & 0.90 & 10.97 & 0.95 & 11.10 & 0.60 & $4.7 \pm 0.2$ & $7.4 \pm 1.5$ & $14.2 \pm 1.6$ \\
\hline G200.34-10.97North & $\leq 0.27$ & $\cdots$ & $\ldots$ & $\leq 0.25$ & $\ldots$ & $\ldots$ & 0.27 & 13.58 & 0.77 & 1.37 & 13.36 & 0.51 & 13.40 & 0.45 & $7.7 \pm 0.5$ & $2.8 \pm 1.2$ & $14.0 \pm 1.5$ \\
\hline G200.34-10.97South & $\leq 0.26$ & $\cdots$ & $\ldots$ & $\leq 0.26$ & $\cdots$ & $\ldots$ & 0.37 & 13.63 & 0.53 & 0.64 & 13.60 & 1.12 & 13.60 & 0.55 & $4.5 \pm 0.3$ & $5.1 \pm 2.1$ & $8.9 \pm 1.5$ \\
\hline G201.52-11.08 & $\leq 0.26$ & $\cdots$ & $\cdots$ & $\leq 0.27$ & $\cdots$ & $\cdots$ & $\leq 0.27$ & $\cdots$ & $\cdots$ & $\leq 0.27$ & $\cdots$ & $\cdots$ & $\cdots$ & $\cdots$ & $\cdots$ & $\cdots$ & $\cdots$ \\
\hline G201.72-11.22 & $\leq 0.26$ & $\ldots$ & $\ldots$ & $\leq 0.26$ & $\ldots$ & $\ldots$ & 0.66 & 9.44 & 0.30 & 0.92 & 9.47 & 0.29 & 9.46 & 0.30 & $6.2 \pm 0.5$ & $2.7 \pm 1.7$ & $9.6 \pm 1.4$ \\
\hline G203.21-11.20East 1 & 0.29 & 10.69 & 0.79 & 0.28 & 10.19 & 0.58 & 0.51 & 10.49 & 0.55 & 0.65 & 10.15 & 1.43 & 10.30 & 0.76 & $4.2 \pm 0.2$ & $6.5 \pm 1.5$ & $9.6 \pm 1.2$ \\
\hline G203.21-11.20East2 & $\leq 0.26$ & $\cdots$ & $\ldots$ & 0.24 & 10.02 & 0.24 & 0.25 & 10.21 & 0.46 & 0.73 & 10.19 & 0.35 & 10.20 & 0.44 & $4.3 \pm 0.2$ & $7.1 \pm 1.7$ & $11.0 \pm 1.3$ \\
\hline G203.21-11.20West1 & $\leq 0.25$ & $\ldots$ & $\ldots$ & $\leq 0.22$ & $\ldots$ & $\ldots$ & 0.34 & 10.66 & 0.70 & 1.02 & 10.70 & 1.02 & 10.60 & 0.50 & $4.9 \pm 0.1$ & $11.2 \pm 0.3$ & $24.2 \pm 0.7$ \\
\hline G203.21-11.20West2 & 0.24 & 10.15 & 0.52 & $\leq 0.23$ & $\ldots$ & $\ldots$ & 0.52 & 10.05 & 0.39 & 0.91 & 10.11 & 0.72 & 10.10 & 0.50 & $4.4 \pm 0.2$ & $12.6 \pm 0.5$ & $20.6 \pm 2.7$ \\
\hline G204.4-11.3A2East & 0.31 & 1.71 & 0.35 & 0.37 & 1.56 & 0.41 & 1.49 & 1.61 & 0.47 & 1.59 & 1.54 & 0.66 & 1.53 & 0.47 & $6.3 \pm 0.2$ & $7.1 \pm 0.9$ & $25.2 \pm 1.6$ \\
\hline G204.4-11.3A2West & $\leq 0.28$ & $\cdots$ & $\cdots$ & $\leq 0.23$ & $\ldots$ & $\cdots$ & 0.30 & 1.44 & 0.89 & 0.61 & 1.12 & 0.44 & 1.43 & 0.83 & $4.0 \pm 0.2$ & $4.7 \pm 2.0$ & $5.7 \pm 1.1$ \\
\hline G205.46-14.56Middle1 & $\leq 0.29$ & $\cdots$ & $\cdots$ & $\leq 0.29$ & $\cdots$ & $\cdots$ & 0.34 & 10.06 & 0.82 & 0.89 & 9.92 & 1.34 & $\cdots$ & $\cdots$ & $6.2 \pm 3.1^{\dagger}$ & $\cdots$ & $\cdots$ \\
\hline G205.46-14.56Middle2 & $\leq 0.19$ & $\ldots$ & $\ldots$ & $\leq 0.20$ & $\ldots$ & $\ldots$ & 0.34 & 10.17 & 0.89 & 1.04 & 10.29 & 0.75 & 10.20 & 0.70 & $6.7 \pm 0.3$ & $2.4 \pm 0.7$ & $9.5 \pm 0.7$ \\
\hline G205.46-14.56Middle3 & $\leq 0.18$ & $\cdots$ & $\cdots$ & $\leq 0.21$ & $\cdots$ & $\cdots$ & 0.30 & 10.11 & 0.96 & 1.28 & 10.05 & 1.07 & 10.10 & 0.78 & $10.0 \pm 0.5$ & $1.5 \pm 0.5$ & $10.6 \pm 0.7$ \\
\hline G205.46-14.56North3 & $\leq 0.18$ & $\cdots$ & $\ldots$ & $\leq 0.20$ & $\cdots$ & $\ldots$ & $\leq 0.23$ & $\ldots$ & $\ldots$ & 1.24 & 9.99 & 1.18 & 9.99 & 0.73 & $8.3 \pm 0.4$ & $2.1 \pm 0.6$ & $11.7 \pm 0.7$ \\
\hline G205.46-14.56North2 & $\leq 0.19$ & $\cdots$ & $\cdots$ & $\leq 0.20$ & $\cdots$ & $\cdots$ & 0.39 & 9.89 & 0.68 & 0.91 & 10.05 & 1.26 & 10.00 & 0.88 & $11.2 \pm 0.3$ & $0.8 \pm 0.1$ & $7.0 \pm 0.2$ \\
\hline G205.46-14.56North1 & $\leq 0.20$ & $\cdots$ & $\cdots$ & $\leq 0.21$ & $\cdots$ & $\cdots$ & 0.33 & 10.02 & 0.67 & 1.78 & 10.00 & 0.73 & 9.99 & 0.58 & $7.8 \pm 0.2$ & $4.3 \pm 0.6$ & $21.7 \pm 1.1$ \\
\hline G205.46-14.56South1 & 0.22 & 10.54 & 0.45 & 0.23 & 9.96 & 0.20 & 0.56 & 10.44 & 0.98 & 2.29 & 10.31 & 1.23 & 10.30 & 1.18 & $10.8 \pm 0.3$ & $2.1 \pm 0.2$ & $17.1 \pm 0.6$ \\
\hline G205.46-14.56South2 & 0.29 & 10.52 & 0.48 & $\leq 0.23$ & $\cdots$ & $\cdots$ & 0.85 & 10.53 & 0.52 & 1.80 & 10.44 & 0.54 & 10.50 & 0.46 & $8.8 \pm 0.3$ & $3.2 \pm 0.5$ & $19.5 \pm 0.9$ \\
\hline G205.46-14.56South3 & $\leq 0.19$ & $\cdots$ & $\cdots$ & $\leq 0.23$ & $\cdots$ & $\cdots$ & 0.35 & 10.37 & 0.63 & 1.33 & 10.36 & 0.81 & 10.40 & 0.61 & $7.5 \pm 0.3$ & $2.8 \pm 0.6$ & $13.5 \pm 0.8$ \\
\hline G206.12-15.76 & $\ldots$ & $\ldots$ & $\ldots$ & $\ldots$ & $\ldots$ & $\ldots$ & $\ldots$ & $\ldots$ & $\ldots$ & $\ldots$ & $\ldots$ & $\ldots$ & $\ldots$ & $\ldots$ & $\ldots$ & $\ldots$ & $\ldots$ \\
\hline G206.21-16.17North & 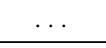 & $\ldots$ & $\ldots$ & 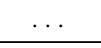 & $\ldots$ & $\ldots$ & . & $\ldots$ & $\ldots$ & $\ldots$ & - & $\ldots$ & $\cdots$ & . & $\ldots$ & $\ldots$ & $\ldots$ \\
\hline
\end{tabular}


Table 3 (continued)

\begin{tabular}{|c|c|c|c|c|c|c|c|c|c|c|c|c|c|c|c|c|c|}
\hline \multirow{3}{*}{ SCUBA-2 core } & \multicolumn{3}{|c|}{$82 \mathrm{GHz} \mathrm{CCS}$} & \multicolumn{3}{|c|}{$94 \mathrm{GHz} \mathrm{CCS}$} & \multicolumn{3}{|c|}{$\mathrm{HC}_{3} \mathrm{~N}$} & \multicolumn{8}{|c|}{$\mathrm{N}_{2} \mathrm{H}^{+}$} \\
\hline & \multirow{2}{*}{$\begin{array}{c}T_{\text {peak }} \\
(\mathrm{K})\end{array}$} & $V_{\mathrm{LSR}}$ & $\Delta v$ & \multirow{2}{*}{$\begin{array}{c}T_{\text {peak }} \\
(\mathrm{K})\end{array}$} & $V_{\mathrm{LSR}}$ & $\Delta v$ & \multirow{2}{*}{$\begin{array}{c}T_{\text {peak }} \\
(\mathrm{K})\end{array}$} & $V_{\mathrm{LSR}}$ & $\Delta v$ & \multirow{2}{*}{$\begin{array}{c}T_{\text {peak }} \\
(\mathrm{K})\end{array}$} & $V_{\mathrm{LSR}, \mathrm{GA}}$ & $\Delta v_{\mathrm{GA}}$ & $V_{\mathrm{LSR}, \mathrm{HFS}}$ & $\Delta v_{\mathrm{HFS}}$ & \multirow{2}{*}{$\begin{array}{l}T_{\mathrm{ex}} \\
(\mathrm{K})\end{array}$} & \multirow[t]{2}{*}{$\tau$} & \multirow{2}{*}{$\begin{array}{c}T_{\text {ant }} \tau \\
(\mathrm{K})\end{array}$} \\
\hline & & \multicolumn{2}{|c|}{$\left(\mathrm{km} \mathrm{s}^{-1}\right)$} & & \multicolumn{2}{|c|}{$\left(\mathrm{km} \mathrm{s}^{-1}\right)$} & & \multicolumn{2}{|c|}{$\left(\mathrm{km} \mathrm{s}^{-1}\right)$} & & & \multicolumn{2}{|c|}{$\left(\mathrm{km} \mathrm{s}^{-1}\right)$} & & & & \\
\hline (1) & (2) & (3) & $(4)$ & (5) & (6) & $(7)$ & (8) & (9) & $(10)$ & $(11)$ & $(12)$ & (13) & (14) & $(15)$ & $(16)$ & $(17)$ & (18) \\
\hline G206.69-16.60North & $\leq 0.24$ & $\cdots$ & $\cdots$ & $\leq 0.23$ & $\cdots$ & $\cdots$ & 0.25 & 11.45 & 0.47 & 0.95 & 11.35 & 0.39 & 11.30 & 0.40 & $6.5 \pm 0.4$ & $2.5 \pm 1.4$ & $9.1 \pm 1.1$ \\
\hline G206.69-16.60South & $\leq 0.24$ & $\cdots$ & $\cdots$ & $\leq 0.22$ & $\cdots$ & $\cdots$ & 0.27 & 12.14 & 0.26 & 0.63 & 12.08 & 1.06 & 12.00 & 0.54 & $4.4 \pm 0.3$ & $5.1 \pm 1.9$ & $8.7 \pm 1.3$ \\
\hline G206.93-16.61East1 & $\leq 0.21$ & $\cdots$ & $\cdots$ & $\leq 0.23$ & $\cdots$ & $\cdots$ & 0.22 & 9.77 & 0.65 & 1.91 & 9.99 & 0.76 & 9.84 & 0.79 & $8.4 \pm 0.1$ & $3.4 \pm 0.1$ & $19.0 \pm 0.1$ \\
\hline G206.93-16.61East2 & $\leq 0.23$ & $\cdots$ & $\ldots$ & $\leq 0.20$ & $\ldots$ & $\ldots$ & $\leq 0.20$ & $\ldots$ & $\ldots$ & 0.53 & 9.76 & 0.58 & 9.78 & 0.60 & $8.4 \pm 4.2^{\dagger}$ & $\ldots$ & $3.7 \pm 0.2$ \\
\hline G206.93-16.61West1 & $\leq 0.23$ & $\cdots$ & $\cdots$ & $\leq 0.21$ & $\cdots$ & $\cdots$ & 0.59 & 9.19 & 0.76 & 1.12 & 9.32 & 0.74 & 9.44 & 0.84 & $11.8 \pm 0.2$ & $0.9 \pm 0.1$ & $8.1 \pm 0.2$ \\
\hline G206.93-16.61West3 & $\leq 0.21$ & $\cdots$ & $\cdots$ & $\leq 0.22$ & $\cdots$ & $\cdots$ & 1.40 & 9.36 & 0.64 & 1.98 & 9.25 & 1.02 & 9.28 & 0.65 & $7.2 \pm 0.2$ & $6.5 \pm 0.6$ & $28.8 \pm 1.4$ \\
\hline G206.93-16.61West4 & $\leq 0.22$ & $\cdots$ & $\cdots$ & $\leq 0.21$ & $\cdots$ & $\cdots$ & $\leq 0.20$ & $\cdots$ & $\cdots$ & 0.86 & 10.19 & 0.51 & 10.10 & 0.64 & $7.2 \pm 0.6$ & $1.6 \pm 1.2$ & $6.9 \pm 0.9$ \\
\hline G206.93-16.61West5 & $\leq 0.19$ & $\cdots$ & $\cdots$ & $\leq 0.22$ & $\cdots$ & $\cdots$ & $\leq 0.20$ & $\cdots$ & $\cdots$ & 1.01 & 9.01 & 0.42 & 9.11 & 0.81 & $5.8 \pm 0.3$ & $2.7 \pm 0.8$ & $8.2 \pm 0.8$ \\
\hline G206.93-16.61West6 & $\leq 0.22$ & $\cdots$ & $\cdots$ & $\leq 0.23$ & $\cdots$ & $\cdots$ & $\leq 0.18$ & $\cdots$ & $\cdots$ & $\leq 0.22$ & $\cdots$ & $\cdots$ & $\cdots$ & $\cdots$ & $\cdots$ & $\cdots$ & $\cdots$ \\
\hline G206.93-16.61West6 & $\leq 0.22$ & $\cdots$ & $\cdots$ & $\leq 0.23$ & $\cdots$ & $\cdots$ & $\leq 0.18$ & $\cdots$ & $\cdots$ & 1.00 & 10.34 & 0.69 & 10.40 & 0.62 & $6.6 \pm 0.3$ & $2.1 \pm 0.9$ & $8.1 \pm 0.7$ \\
\hline G207.36-19.82North1 & $\leq 0.22$ & $\cdots$ & $\cdots$ & $\leq 0.23$ & $\cdots$ & $\cdots$ & 0.43 & 10.63 & 1.01 & 0.96 & 10.52 & 1.48 & 10.70 & 1.13 & $10.3 \pm 0.1$ & $0.9 \pm 0.1$ & $6.7 \pm 0.1$ \\
\hline G207.36-19.82North2 & $\leq 0.22$ & $\cdots$ & $\ldots$ & $\leq 0.20$ & $\ldots$ & $\ldots$ & 0.21 & 11.06 & 1.05 & 1.03 & 11.25 & 0.51 & 11.20 & 0.45 & $4.9 \pm 0.2$ & $6.6 \pm 1.3$ & $14.3 \pm 1.4$ \\
\hline G207.36-19.82North3 & $\leq 0.22$ & $\cdots$ & $\cdots$ & $\leq 0.22$ & $\cdots$ & $\cdots$ & 0.47 & 11.08 & 0.10 & 0.74 & 11.05 & 0.45 & 11.20 & 0.69 & $4.5 \pm 0.2$ & $4.0 \pm 1.4$ & $7.2 \pm 1.0$ \\
\hline G207.36-19.82North4 & $\leq 0.23$ & $\cdots$ & $\cdots$ & $\leq 0.20$ & $\cdots$ & $\cdots$ & $\leq 0.21$ & $\cdots$ & $\cdots$ & 0.65 & 11.01 & 0.65 & 11.20 & 0.80 & $4.4 \pm 0.3$ & $3.6 \pm 1.5$ & $6.0 \pm 1.0$ \\
\hline G207.36-19.82South & $\leq 0.22$ & $\cdots$ & $\cdots$ & $\leq 0.23$ & $\cdots$ & $\cdots$ & 0.24 & 11.19 & 0.68 & 0.74 & 11.24 & 0.43 & 11.30 & 0.38 & $4.5 \pm 0.2$ & $5.9 \pm 1.8$ & $10.7 \pm 1.4$ \\
\hline G207.3-19.8A2North1 & $\leq 0.22$ & $\cdots$ & $\ldots$ & $\leq 0.23$ & $\ldots$ & $\ldots$ & 0.23 & 11.85 & 0.16 & 0.43 & 11.87 & 0.39 & $\ldots$ & $\ldots$ & $5.9 \pm 3.0^{\dagger}$ & $\ldots$ & $\ldots$ \\
\hline G207.3-19.8A2North2 & $\leq 0.23$ & $\cdots$ & $\cdots$ & $\leq 0.22$ & $\cdots$ & $\cdots$ & $\leq 0.21$ & $\cdots$ & $\cdots$ & 0.52 & 12.60 & 0.33 & 12.60 & 0.42 & $5.9 \pm 3.0^{\dagger}$ & $\ldots$ & $3.5 \pm 0.3$ \\
\hline G207.3-19.8A2North3 & $\leq 0.22$ & $\cdots$ & $\cdots$ & $\leq 0.21$ & $\cdots$ & $\cdots$ & $\leq 0.19$ & $\cdots$ & $\cdots$ & $\leq 0.22$ & $\cdots$ & $\cdots$ & $\cdots$ & $\cdots$ & $\cdots$ & $\cdots$ & $\cdots$ \\
\hline G207.3-19.8A2South & $\leq 0.23$ & $\cdots$ & $\cdots$ & $\leq 0.21$ & $\cdots$ & $\cdots$ & $\leq 0.18$ & $\cdots$ & $\cdots$ & 0.62 & 11.82 & 0.30 & 11.80 & 0.45 & $6.7 \pm 0.7$ & $1.2 \pm 2.1$ & $4.7 \pm 0.9$ \\
\hline G208.68-19.20North1 & $\leq 0.23$ & $\cdots$ & $\ldots$ & $\leq 0.21$ & $\ldots$ & $\ldots$ & 1.18 & 11.12 & 0.82 & 1.92 & 11.13 & 0.96 & 11.20 & 0.72 & $9.8 \pm 0.3$ & $2.7 \pm 0.4$ & $19.2 \pm 0.9$ \\
\hline G208.68-19.20North2 & $\leq 0.24$ & $\cdots$ & $\cdots$ & $\leq 0.21$ & $\cdots$ & $\cdots$ & 0.61 & 11.14 & 0.77 & 4.90 & 11.14 & 0.67 & 11.10 & 0.44 & $12.4 \pm 0.2$ & $11.3 \pm 0.3$ & $109.0 \pm 1.9$ \\
\hline G208.68-19.20North3 & $\leq 0.22$ & $\cdots$ & $\cdots$ & $\leq 0.25$ & $\cdots$ & $\cdots$ & 0.76 & 11.25 & 1.03 & 4.19 & 11.12 & 0.96 & 11.10 & 0.69 & $11.8 \pm 0.2$ & $6.8 \pm 0.3$ & $61.8 \pm 1.5$ \\
\hline G208.68-19.20South & $\leq 0.22$ & $\cdots$ & $\cdots$ & $\leq 0.22$ & $\cdots$ & $\cdots$ & 0.50 & 10.50 & 0.90 & 2.25 & 10.35 & 0.94 & 10.30 & 0.95 & $8.2 \pm 0.1$ & $4.1 \pm 0.1$ & $22.1 \pm 0.1$ \\
\hline G208.89-20.04East & $\leq 0.29$ & $\ldots$ & $\ldots$ & $\leq 0.31$ & $\ldots$ & $\ldots$ & 1.10 & 8.77 & 0.53 & 2.05 & 8.74 & 0.52 & 8.74 & 0.38 & $6.5 \pm 0.3$ & $11.2 \pm 1.4$ & $42.1 \pm 3.3$ \\
\hline G209.05-19.73North & $\leq 0.32$ & $\cdots$ & $\cdots$ & $\leq 0.33$ & $\cdots$ & $\cdots$ & $\leq 0.29$ & $\cdots$ & $\cdots$ & 0.75 & 8.27 & 0.37 & 8.25 & 0.38 & $8.8 \pm 0.2$ & $1.2 \pm 0.1$ & $7.1 \pm 0.2$ \\
\hline G209.05-19.73South & $\leq 0.34$ & $\cdots$ & $\cdots$ & $\leq 0.33$ & $\cdots$ & $\cdots$ & 0.44 & 7.84 & 0.38 & 0.71 & 7.87 & 0.45 & 7.89 & 0.43 & $9.5 \pm 0.4$ & $0.9 \pm 0.1$ & $6.3 \pm 0.4$ \\
\hline G209.29-19.65North1 & $\leq 0.23$ & $\cdots$ & $\cdots$ & $\leq 0.23$ & $\cdots$ & $\cdots$ & $\leq 0.19$ & $\cdots$ & $\cdots$ & 1.80 & 8.46 & 0.46 & $\cdots$ & $\cdots$ & $8.7 \pm 4.3^{\dagger}$ & $\cdots$ & $\cdots$ \\
\hline G209.29-19.65North2 & $\leq 0.22$ & $\ldots$ & $\ldots$ & $\leq 0.23$ & $\ldots$ & $\ldots$ & $\leq 0.21$ & $\ldots$ & $\ldots$ & 0.99 & 7.42 & 0.34 & 7.43 & 0.31 & $6.3 \pm 0.1$ & $2.8 \pm 0.4$ & $9.9 \pm 0.3$ \\
\hline G209.29-19.65North2 & $\leq 0.23$ & $\cdots$ & $\ldots$ & $\leq 0.23$ & $\cdots$ & $\cdots$ & $\leq 0.21$ & $\cdots$ & $\cdots$ & 1.96 & 8.88 & 0.29 & 8.88 & 0.28 & $8.6 \pm 4.3^{\dagger}$ & $\cdots$ & $15.1 \pm 0.2$ \\
\hline G209.29-19.65North3 & $\leq 0.24$ & $\cdots$ & $\cdots$ & $\leq 0.20$ & $\cdots$ & $\cdots$ & $\leq 0.20$ & $\cdots$ & $\cdots$ & 0.79 & 8.72 & 0.45 & 8.68 & 0.64 & $10.0 \pm 1.1$ & $0.7 \pm 1.4$ & $5.1 \pm 0.8$ \\
\hline G209.29-19.65South1 & $\leq 0.20$ & $\cdots$ & $\cdots$ & $\leq 0.21$ & $\cdots$ & $\cdots$ & $\leq 0.20$ & $\cdots$ & $\cdots$ & 1.35 & 7.77 & 0.98 & 7.68 & 1.42 & $7.3 \pm 0.3$ & $2.2 \pm 0.4$ & $10.0 \pm 0.6$ \\
\hline G209.29-19.65South2 & $\leq 0.20$ & $\ldots$ & $\ldots$ & $\leq 0.22$ & $\ldots$ & $\ldots$ & $\leq 0.20$ & $\cdots$ & $\cdots$ & 1.02 & 7.64 & 0.95 & 7.84 & 1.12 & $4.9 \pm 0.2$ & $4.5 \pm 0.7$ & $9.9 \pm 0.8$ \\
\hline G209.29-19.65South2 & $\leq 0.21$ & $\cdots$ & $\cdots$ & $\leq 0.23$ & $\cdots$ & $\cdots$ & $\leq 0.20$ & $\cdots$ & $\cdots$ & 1.35 & 8.91 & 0.79 & 8.98 & 0.38 & $4.2 \pm 0.2$ & $11.3 \pm 2.2$ & $16.7 \pm 2.1$ \\
\hline G209.29-19.65South3 & $\leq 0.20$ & $\ldots$ & $\cdots$ & $\leq 0.23$ & $\cdots$ & $\cdots$ & $\leq 0.20$ & $\cdots$ & $\cdots$ & 1.03 & 7.73 & 0.47 & 7.71 & 0.66 & $5.8 \pm 0.3$ & $3.0 \pm 1.1$ & $9.1 \pm 1.0$ \\
\hline G209.55-19.68North1 & 0.42 & 7.74 & 0.15 & $\leq 0.21$ & $\cdots$ & $\cdots$ & $\leq 0.21$ & $\cdots$ & $\cdots$ & 1.76 & 7.20 & 1.30 & 7.14 & 0.84 & $6.7 \pm 0.2$ & $5.6 \pm 0.5$ & $22.3 \pm 1.2$ \\
\hline G209.55-19.68North2 & $\leq 0.20$ & $\cdots$ & $\cdots$ & $\leq 0.23$ & $\cdots$ & $\ldots$ & $\leq 0.22$ & $\ldots$ & $\ldots$ & 1.21 & 8.22 & 0.31 & 8.22 & 0.29 & $13.0 \pm 0.3$ & $1.0 \pm 0.1$ & $10.6 \pm 0.3$ \\
\hline
\end{tabular}


Table 3 (continued)

\begin{tabular}{|c|c|c|c|c|c|c|c|c|c|c|c|c|c|c|c|c|c|}
\hline \multirow{3}{*}{ SCUBA-2 core } & \multicolumn{3}{|c|}{$82 \mathrm{GHz} \mathrm{CCS}$} & \multicolumn{3}{|c|}{$94 \mathrm{GHz} \mathrm{CCS}$} & \multicolumn{3}{|c|}{$\mathrm{HC}_{3} \mathrm{~N}$} & \multicolumn{8}{|c|}{$\mathrm{N}_{2} \mathrm{H}^{+}$} \\
\hline & \multirow{2}{*}{$\begin{array}{c}T_{\text {peak }} \\
(\mathrm{K})\end{array}$} & $V_{\mathrm{LSR}}$ & $\Delta v$ & \multirow{2}{*}{$\begin{array}{c}T_{\text {peak }} \\
(\mathrm{K})\end{array}$} & $V_{\mathrm{LSR}}$ & $\Delta v$ & \multirow{2}{*}{$\begin{array}{c}T_{\text {peak }} \\
(\mathrm{K})\end{array}$} & $V_{\mathrm{LSR}}$ & $\Delta v$ & \multirow{2}{*}{$\begin{array}{c}T_{\text {peak }} \\
(\mathrm{K})\end{array}$} & \multirow[t]{2}{*}{$V_{\mathrm{LSR}, \mathrm{GA}}$} & $\Delta v_{\mathrm{GA}}$ & $V_{\mathrm{LSR}, \mathrm{HFS}}$ & $\Delta v_{\mathrm{HFS}}$ & \multirow{3}{*}{$\begin{array}{l}T_{\text {ex }} \\
(\mathrm{K}) \\
(16)\end{array}$} & \multirow[t]{2}{*}{$\tau$} & \multirow{3}{*}{$\begin{array}{r}T_{\text {ant }} \tau \\
(\mathrm{K}) \\
(18)\end{array}$} \\
\hline & & \multicolumn{2}{|c|}{$\left(\mathrm{km} \mathrm{s}^{-1}\right)$} & & \multicolumn{2}{|c|}{$\left(\mathrm{km} \mathrm{s}^{-1}\right)$} & & \multicolumn{2}{|c|}{$\left(\mathrm{km} \mathrm{s}^{-1}\right)$} & & & \multicolumn{2}{|c|}{$\left(\mathrm{km} \mathrm{s}^{-1}\right)$} & & & & \\
\hline$(1)$ & $(2)$ & (3) & $(4)$ & $(5)$ & (6) & $(7)$ & $(8)$ & (9) & $(10)$ & $(11)$ & $(12)$ & $(13)$ & $(14)$ & $(15)$ & & $(17)$ & \\
\hline G209.55-19.68North3 & 0.21 & 7.90 & 0.36 & $\leq 0.24$ & $\cdots$ & $\cdots$ & 0.32 & 8.08 & 0.50 & 1.22 & 7.99 & 0.71 & 7.99 & 0.50 & $5.9 \pm 0.3$ & $5.1 \pm 1.0$ & $15.9 \pm 1.3$ \\
\hline G209.55-19.68South1 & $\leq 0.23$ & $\cdots$ & $\cdots$ & $\leq 0.23$ & $\cdots$ & $\cdots$ & 0.31 & 7.43 & 0.60 & 1.37 & 7.35 & 0.94 & 7.37 & 0.68 & $6.0 \pm 0.2$ & $6.0 \pm 0.7$ & $19.5 \pm 1.2$ \\
\hline G209.55-19.68South3 & $\leq 0.22$ & $\cdots$ & $\cdots$ & $\leq 0.24$ & $\cdots$ & $\cdots$ & 0.45 & 8.12 & 0.55 & 2.08 & 8.11 & 0.51 & 8.12 & 0.45 & $7.6 \pm 0.2$ & $5.9 \pm 0.7$ & $28.5 \pm 1.4$ \\
\hline G209.77-19.40East1 & 0.26 & 8.04 & 0.25 & $\leq 0.23$ & $\cdots$ & $\cdots$ & 0.48 & 8.05 & 0.48 & 2.58 & 8.03 & 0.46 & 8.02 & 0.35 & $8.0 \pm 0.2$ & $8.5 \pm 0.6$ & $45.3 \pm 1.9$ \\
\hline G209.77-19.40East2 & $\leq 0.20$ & $\ldots$ & $\ldots$ & 0.27 & 8.19 & 0.23 & 0.48 & 7.99 & 0.42 & 1.38 & 7.99 & 0.65 & 8.01 & 0.47 & $6.7 \pm 0.3$ & $4.0 \pm 0.9$ & $16.2 \pm 1.2$ \\
\hline G209.77-19.40East3 & $\leq 0.21$ & $\cdots$ & $\cdots$ & $\leq 0.21$ & $\cdots$ & $\cdots$ & 0.30 & 7.76 & 0.26 & 1.32 & 7.73 & 0.25 & 7.75 & 0.22 & $5.3 \pm 0.3$ & $5.3 \pm 1.6$ & $13.9 \pm 1.6$ \\
\hline G209.77-19.40East3 & $\leq 0.21$ & $\ldots$ & $\ldots$ & $\leq 0.22$ & $\ldots$ & $\ldots$ & 0.25 & 8.26 & 0.26 & 0.79 & 8.68 & 0.31 & 8.27 & 0.30 & $7.0 \pm 3.5^{\dagger}$ & $\ldots$ & $5.4 \pm 0.3$ \\
\hline G209.77-19.40West & 0.20 & 8.59 & 0.20 & $\leq 0.22$ & $\cdots$ & $\cdots$ & 0.32 & 8.34 & 0.22 & 1.54 & 8.33 & 0.28 & 8.33 & 0.23 & $6.3 \pm 0.3$ & $6.0 \pm 1.2$ & $21.5 \pm 1.8$ \\
\hline G209.77-19.61East & $\leq 0.22$ & $\ldots$ & $\ldots$ & $\leq 0.23$ & $\ldots$ & $\ldots$ & 0.28 & 7.98 & 0.27 & 0.47 & 8.02 & 0.47 & $\cdots$ & $\cdots$ & $6.6 \pm 3.3^{\dagger}$ & $\ldots$ & $\ldots$ \\
\hline G209.77-19.61West & $\leq 0.23$ & $\cdots$ & $\cdots$ & $\leq 0.23$ & $\cdots$ & $\cdots$ & 0.22 & 7.26 & 0.33 & 0.75 & 7.18 & 0.49 & 7.19 & 0.46 & $6.6 \pm 3.3^{\dagger}$ & $\cdots$ & $5.9 \pm 0.3$ \\
\hline G209.79-19.80East & $\leq 0.22$ & $\ldots$ & $\ldots$ & $\leq 0.24$ & $\ldots$ & $\ldots$ & 0.36 & 5.49 & 0.23 & 0.66 & 5.43 & 0.41 & 5.41 & 0.37 & $5.8 \pm 0.6$ & $2.1 \pm 2.1$ & $6.4 \pm 1.2$ \\
\hline G209.79-19.80West & $\leq 0.23$ & $\cdots$ & $\cdots$ & $\leq 0.24$ & $\cdots$ & $\cdots$ & 0.31 & 5.82 & 0.52 & 1.12 & 5.72 & 0.63 & 5.74 & 0.56 & $5.9 \pm 0.3$ & $3.9 \pm 1.0$ & $12.4 \pm 1.1$ \\
\hline G209.79-19.80West & $\leq 0.23$ & $\ldots$ & $\ldots$ & $\leq 0.24$ & $\ldots$ & $\ldots$ & $\leq 0.21$ & $\cdots$ & $\ldots$ & $\leq 0.23$ & $\cdots$ & $\ldots$ & $\ldots$ & $\cdots$ & $\ldots$ & $\ldots$ & $\ldots$ \\
\hline G209.94-19.52North & 0.39 & 8.13 & 0.13 & 0.27 & 8.10 & 0.22 & 0.73 & 8.02 & 0.57 & 1.45 & 7.95 & 1.27 & 8.03 & 0.60 & $6.3 \pm 0.2$ & $6.4 \pm 0.8$ & $23.0 \pm 1.4$ \\
\hline G209.94-19.52South1 & $\leq 0.26$ & $\ldots$ & $\ldots$ & $\leq 0.22$ & $\ldots$ & $\ldots$ & 0.25 & 7.63 & 0.41 & 1.41 & 7.49 & 0.32 & 7.50 & 0.26 & $4.1 \pm 0.2$ & $12.2 \pm 2.8$ & $17.3 \pm 2.5$ \\
\hline G209.94-19.52South1 & $\leq 0.24$ & $\cdots$ & $\cdots$ & 0.40 & 8.12 & 0.10 & 0.27 & 8.18 & 0.32 & 1.51 & 8.15 & 0.46 & 8.11 & 0.37 & $8.9 \pm 0.5$ & $2.4 \pm 0.9$ & $14.6 \pm 1.2$ \\
\hline G209.94-19.52South2 & $\leq 0.23$ & $\cdots$ & $\cdots$ & $\leq 0.23$ & $\cdots$ & $\cdots$ & 0.25 & 7.35 & 0.61 & 1.09 & 7.31 & 0.64 & 7.31 & 0.59 & $6.5 \pm 0.3$ & $2.9 \pm 0.9$ & $10.8 \pm 1.0$ \\
\hline G210.37-19.53North & $\leq 0.22$ & $\cdots$ & $\cdots$ & $\leq 0.20$ & $\cdots$ & $\cdots$ & 0.28 & 6.42 & 0.24 & 0.72 & 6.37 & 0.35 & 6.38 & 0.27 & $4.2 \pm 0.1$ & $9.4 \pm 1.8$ & $13.5 \pm 1.0$ \\
\hline G210.37-19.53South & $\leq 0.22$ & $\cdots$ & $\cdots$ & $\leq 0.21$ & $\cdots$ & $\cdots$ & 0.48 & 5.99 & 0.91 & 1.00 & 5.61 & 0.69 & 5.68 & 0.72 & $6.5 \pm 0.4$ & $2.2 \pm 0.9$ & $8.4 \pm 0.8$ \\
\hline G210.49-19.79East 1 & 0.25 & 8.62 & 0.47 & $\leq 0.20$ & $\cdots$ & $\cdots$ & 0.84 & 8.87 & 0.90 & 1.35 & 8.86 & 1.06 & 8.84 & 0.76 & $21.8 \pm 0.4$ & $0.6 \pm 0.1$ & $11.2 \pm 0.2$ \\
\hline G210.49-19.79East2 & $\leq 0.21$ & $\cdots$ & $\cdots$ & $\leq 0.23$ & $\cdots$ & $\cdots$ & 0.24 & 8.03 & 0.34 & 1.08 & 8.04 & 0.42 & 8.02 & 0.32 & $5.8 \pm 0.3$ & $4.4 \pm 1.3$ & $13.6 \pm 1.4$ \\
\hline G210.49-19.79East2 & $\leq 0.20$ & $\cdots$ & $\cdots$ & $\leq 0.23$ & $\cdots$ & $\cdots$ & 0.59 & 10.45 & 0.82 & 1.90 & 10.23 & 0.92 & 10.30 & 0.74 & $8.7 \pm 0.3$ & $3.2 \pm 0.4$ & $19.0 \pm 0.9$ \\
\hline G210.49-19.79West & $\leq 0.21$ & $\cdots$ & $\cdots$ & $\leq 0.23$ & $\cdots$ & $\cdots$ & 0.23 & 9.19 & 1.78 & 1.48 & 9.01 & 0.77 & 9.04 & 0.60 & $5.8 \pm 0.2$ & $7.8 \pm 0.8$ & $24.1 \pm 1.5$ \\
\hline G210.82-19.47North1 & $\leq 0.18$ & $\cdots$ & $\cdots$ & $\leq 0.19$ & $\cdots$ & $\cdots$ & $\leq 0.22$ & $\cdots$ & $\cdots$ & 1.30 & 5.29 & 0.49 & 5.30 & 0.44 & $7.7 \pm 0.3$ & $2.7 \pm 0.7$ & $13.5 \pm 0.9$ \\
\hline G210.82-19.47North2 & $\leq 0.14$ & $\cdots$ & $\cdots$ & $\leq 0.15$ & $\cdots$ & $\cdots$ & 0.35 & 5.20 & 0.27 & 1.01 & 5.21 & 0.36 & 5.21 & 0.35 & $21.2 \pm 0.4$ & $0.5 \pm 0.1$ & $8.8 \pm 0.2$ \\
\hline G210.97-19.33North & $\leq 0.17$ & $\cdots$ & $\cdots$ & $\leq 0.15$ & $\cdots$ & $\cdots$ & $\leq 0.15$ & $\cdots$ & $\cdots$ & $\leq 0.16$ & $\cdots$ & $\cdots$ & $\cdots$ & $\cdots$ & $\cdots$ & $\cdots$ & $\cdots$ \\
\hline G210.97-19.33South1 & 0.42 & 2.94 & 0.38 & $\leq 0.23$ & $\cdots$ & $\cdots$ & 0.77 & 2.90 & 0.43 & 0.58 & 2.87 & 0.40 & 2.87 & 0.38 & $4.3 \pm 0.2$ & $5.1 \pm 1.9$ & $8.0 \pm 1.2$ \\
\hline G210.97-19.33South2 & 0.38 & 3.23 & 0.28 & $\leq 0.21$ & $\cdots$ & $\cdots$ & 0.48 & 3.21 & 0.85 & 0.35 & 3.23 & 0.86 & $\cdots$ & $\cdots$ & $6.4 \pm 3.2^{\dagger}$ & $\cdots$ & $\cdots$ \\
\hline G210.97-19.33South2 & $\leq 0.20$ & $\cdots$ & $\cdots$ & $\leq 0.21$ & $\cdots$ & $\cdots$ & 0.31 & 4.49 & 0.40 & 0.38 & 4.24 & 1.09 & 4.54 & 0.41 & $4.3 \pm 0.1$ & $2.0 \pm 0.6$ & $3.2 \pm 0.2$ \\
\hline G211.01-19.54North & 0.27 & 6.10 & 1.17 & 0.27 & 6.12 & 0.87 & 1.07 & 6.07 & 0.76 & 1.89 & 6.03 & 0.79 & 5.86 & 0.83 & $8.7 \pm 0.2$ & $3.1 \pm 0.3$ & $18.7 \pm 0.6$ \\
\hline G211.01-19.54South & 0.27 & 5.83 & 0.95 & 0.19 & 5.87 & 0.90 & 0.63 & 5.87 & 0.82 & 1.62 & 5.72 & 1.01 & 5.76 & 0.70 & $7.2 \pm 0.1$ & $4.5 \pm 0.4$ & $19.8 \pm 0.7$ \\
\hline G211.16-19.33North1 & 0.28 & 3.54 & 0.37 & 0.26 & 3.45 & 0.41 & 1.11 & 3.48 & 0.53 & 0.64 & 3.37 & 0.61 & 3.42 & 0.44 & $4.1 \pm 0.1$ & $7.4 \pm 1.5$ & $10.0 \pm 1.0$ \\
\hline G211.16-19.33North2 & 0.17 & 3.22 & 0.55 & $\leq 0.14$ & $\cdots$ & $\cdots$ & 0.95 & 3.44 & 0.54 & 1.01 & 3.49 & 0.70 & 3.52 & 0.46 & $4.9 \pm 0.1$ & $7.7 \pm 1.0$ & $16.6 \pm 1.1$ \\
\hline G211.16-19.33North3 & 0.18 & 3.28 & 0.40 & $\leq 0.15$ & $\cdots$ & $\cdots$ & 0.70 & 3.39 & 0.39 & 1.03 & 3.31 & 0.45 & 3.33 & 0.35 & $4.7 \pm 0.1$ & $9.9 \pm 1.2$ & $19.5 \pm 1.4$ \\
\hline G211.16-19.33North4 & $\leq 0.16$ & $\ldots$ & $\cdots$ & $\leq 0.15$ & $\cdots$ & $\cdots$ & 0.42 & 4.52 & 0.71 & 0.55 & 4.59 & 1.01 & $\cdots$ & $\cdots$ & $6.2 \pm 3.1^{\dagger}$ & $\cdots$ & $\cdots$ \\
\hline G211.16-19.33North5 & $\leq 0.15$ & $\cdots$ & $\ldots$ & $\leq 0.14$ & $\cdots$ & $\cdots$ & 0.53 & 4.30 & 0.54 & 1.23 & 4.29 & 0.78 & 4.28 & 0.48 & $5.9 \pm 0.2$ & $5.6 \pm 0.6$ & $17.7 \pm 0.9$ \\
\hline
\end{tabular}

Table 3 continued 
Table 3 (continued)

\begin{tabular}{|c|c|c|c|c|c|c|c|c|c|c|c|c|c|c|c|c|c|}
\hline \multirow{3}{*}{ SCUBA-2 core } & \multicolumn{3}{|c|}{$82 \mathrm{GHz} \mathrm{CCS}$} & \multicolumn{3}{|c|}{$94 \mathrm{GHz} \mathrm{CCS}$} & \multicolumn{3}{|c|}{$\mathrm{HC}_{3} \mathrm{~N}$} & \multicolumn{8}{|c|}{$\mathrm{N}_{2} \mathrm{H}^{+}$} \\
\hline & $T_{\text {peak }}$ & $V_{\mathrm{LSR}}$ & $\Delta v$ & \multirow{3}{*}{$\begin{array}{c}T_{\text {peak }} \\
(\mathrm{K}) \\
(5)\end{array}$} & $V_{\mathrm{LSR}}$ & $\Delta v$ & \multirow{3}{*}{$\begin{array}{c}T_{\text {peak }} \\
(\mathrm{K}) \\
(8)\end{array}$} & $V_{\mathrm{LSR}}$ & $\Delta v$ & \multirow{3}{*}{$\begin{array}{l}T_{\text {peak }} \\
(\mathrm{K}) \\
(11)\end{array}$} & \multirow{3}{*}{$\begin{array}{c}V_{\mathrm{LSR}, \mathrm{GA}} \\
(12)\end{array}$} & $\Delta v_{\mathrm{GA}}$ & $V_{\mathrm{LSR}, \mathrm{HFS}}$ & $\Delta v_{\mathrm{HFS}}$ & \multirow{3}{*}{$\begin{array}{l}T_{\text {ex }} \\
(\mathrm{K}) \\
(16)\end{array}$} & \multirow{3}{*}{$\begin{array}{c}\tau \\
(17)\end{array}$} & \multirow{3}{*}{$\begin{array}{c}T_{\text {ant }} \tau \\
\\
(\mathrm{K}) \\
(18)\end{array}$} \\
\hline & $(\mathrm{K})$ & \multicolumn{2}{|c|}{$\left(\mathrm{km} \mathrm{s}^{-1}\right)$} & & \multicolumn{2}{|c|}{$\left(\mathrm{km} \mathrm{s}^{-1}\right)$} & & \multicolumn{2}{|c|}{$\left(\mathrm{km} \mathrm{s}^{-1}\right)$} & & & \multicolumn{2}{|c|}{$\left(\mathrm{km} \mathrm{s}^{-1}\right)$} & & & & \\
\hline (1) & (2) & (3) & (4) & & (6) & $(7)$ & & (9) & (10) & & & (13) & (14) & (15) & & & \\
\hline G211.16-19.33South & $\leq 0.15$ & $\cdots$ & $\cdots$ & $\leq 0.16$ & $\cdots$ & $\cdots$ & $\leq 0.15$ & $\cdots$ & $\cdots$ & 0.18 & 3.11 & 0.67 & 3.18 & 0.49 & $6.2 \pm 3.1^{\dagger}$ & $\cdots$ & $1.2 \pm 0.2$ \\
\hline G211.47-19.27North & $\leq 0.26$ & $\cdots$ & ... & 0.36 & 4.10 & 0.12 & 0.93 & 4.00 & 0.63 & 1.22 & 3.99 & 0.97 & 4.01 & 0.52 & $5.0 \pm 0.1$ & $12.7 \pm 0.3$ & $28.6 \pm 0.6$ \\
\hline G211.47-19.27South & 0.32 & 5.42 & 0.89 & $\leq 0.23$ & $\ldots$ & $\ldots$ & 0.78 & 5.31 & 1.16 & 1.67 & 5.52 & 0.93 & 5.34 & 1.03 & $7.7 \pm 0.3$ & $2.8 \pm 0.5$ & $13.7 \pm 0.9$ \\
\hline G211.72-19.25North & $\leq 0.25$ & $\cdots$ & $\ldots$ & $\leq 0.23$ & $\ldots$ & $\ldots$ & 0.66 & 3.51 & 0.43 & 1.16 & 3.49 & 0.44 & 3.48 & 0.38 & $5.9 \pm 0.3$ & $4.4 \pm 1.3$ & $14.0 \pm 1.5$ \\
\hline G211.72-19.25South1 & 0.53 & 4.65 & 0.24 & $\leq 0.27$ & $\ldots$ & $\ldots$ & 0.59 & 4.28 & 0.19 & 0.42 & 4.31 & 0.42 & 4.32 & 0.40 & $8.4 \pm 0.5$ & $0.7 \pm 0.1$ & $4.2 \pm 0.4$ \\
\hline G212.10-19.15North1 & $\leq 0.26$ & $\ldots$ & $\ldots$ & $\leq 0.24$ & $\cdots$ & .. & 0.43 & 4.33 & 0.96 & 0.80 & 4.22 & 0.78 & 4.34 & 0.84 & $4.6 \pm 0.2$ & $4.9 \pm 1.2$ & $9.0 \pm 1.1$ \\
\hline G212.10-19.15North2 & $\leq 0.24$ & $\ldots$ & $\ldots$ & $\leq 0.25$ & $\ldots$ & $\cdots$ & 0.50 & 4.39 & 0.86 & 1.24 & 4.45 & 1.04 & 4.48 & 0.69 & $5.2 \pm 0.2$ & $8.0 \pm 1.1$ & $19.7 \pm 1.6$ \\
\hline G212.10-19.15North3 & 1.30 & 4.30 & 0.53 & 0.94 & 4.25 & 0.37 & 2.06 & 4.20 & 0.63 & 0.82 & 4.13 & 1.30 & 4.16 & 0.55 & $4.3 \pm 0.2$ & $12.7 \pm 0.5$ & $19.5 \pm 2.5$ \\
\hline G212.10-19.15South & $\leq 0.26$ & $\ldots$ & ... & $\leq 0.26$ & $\ldots$ & $\ldots$ & 0.43 & 4.02 & 0.61 & 1.28 & 3.78 & 0.74 & 3.48 & 0.38 & $5.9 \pm 0.4$ & $4.4 \pm 1.4$ & $14.0 \pm 1.6$ \\
\hline G212.84-19.45North & 0.63 & 4.40 & 0.24 & 0.40 & 4.32 & 0.31 & 1.55 & 4.34 & 0.38 & 1.00 & 4.31 & 0.42 & 4.31 & 0.34 & $4.5 \pm 0.2$ & $11.5 \pm 2.5$ & $19.9 \pm 2.6$ \\
\hline G212.84-19.45South & $\leq 0.25$ & $\cdots$ & $\cdots$ & $\leq 0.26$ & $\ldots$ & $\ldots$ & 0.70 & 4.46 & 0.26 & 0.41 & 4.40 & 0.40 & $\cdots$ & $\cdots$ & $5.9 \pm 3.0^{\dagger}$ & $\cdots$ & $\cdots$ \\
\hline G215.44-16.38 & $\leq 0.25$ & $\cdots$ & $\cdots$ & 0.41 & 11.40 & 0.11 & 0.96 & 11.38 & 0.49 & 0.74 & 11.42 & 0.42 & 11.40 & 0.34 & $4.6 \pm 0.3$ & $6.3 \pm 2.1$ & $11.6 \pm 1.7$ \\
\hline G215.87-17.62North & $\leq 0.23$ & $\ldots$ & $\cdots$ & $\leq 0.22$ & $\ldots$ & $\ldots$ & $\leq 0.21$ & $\cdots$ & $\ldots$ & 0.26 & 9.28 & 0.89 & 9.26 & 0.46 & $4.2 \pm 0.6$ & $2.0 \pm 4.0$ & $3.0 \pm 1.2$ \\
\hline G215.87-17.62Middle & 0.28 & 8.92 & 0.37 & 0.25 & 8.86 & 0.20 & 0.43 & 8.99 & 0.41 & 0.97 & 8.96 & 0.46 & 8.95 & 0.35 & $4.9 \pm 0.2$ & $7.3 \pm 1.6$ & $15.5 \pm 1.7$ \\
\hline G215.87-17.62South & $\leq 0.23$ & $\ldots$ & $\ldots$ & $\leq 0.22$ & $\ldots$ & $\ldots$ & 0.66 & 9.90 & 0.55 & 0.49 & 9.98 & 1.01 & $\ldots$ & $\ldots$ & $6.1 \pm 3.0^{\dagger}$ & $\ldots$ & $\cdots$ \\
\hline
\end{tabular}

NoтE-All values are measured in a spectrum whose peak temperature is higher than $3 \sigma$. In the case of a peak temperature below $3 \sigma$, the $3 \sigma$ level is listed as an upper limit. Column 1: SCUBA-2 core name, Column 2-4: Peak temperature at the $T_{\mathrm{A}}$ scale, systemic velocity, Full Width at of $94 \mathrm{GHz}$ CCS line, Column 8-10. Same as Column $2-4$ but of $\mathrm{HC}_{3} \mathrm{~N}$, Column 11-13. Peak temperature at the $T_{\mathrm{A}}^{*}$ scale, systemic velocity, and FWHM the enpe hyperfices that the value is derived from dust temperature. 
Table 4. Properties of c- $\mathrm{C}_{3} \mathrm{H}_{2}$, DNC, $\mathrm{HN}^{13} \mathrm{C}$, and $\mathrm{N}_{2} \mathrm{D}^{+}$lines

\begin{tabular}{|c|c|c|c|c|c|c|c|c|c|c|c|c|c|c|c|c|c|}
\hline \multirow{3}{*}{ SCUBA-2 core } & \multicolumn{3}{|c|}{$\mathrm{c}-\mathrm{C}_{3} \mathrm{H}_{2}$} & \multicolumn{3}{|c|}{$\mathrm{DNC}$} & \multicolumn{3}{|c|}{$\mathrm{HN}^{13} \mathrm{C}$} & \multicolumn{8}{|c|}{$\mathrm{N}_{2} \mathrm{D}^{+}$} \\
\hline & \multirow{2}{*}{$\begin{array}{c}T_{\text {peak }} \\
(\mathrm{K})\end{array}$} & $V_{\mathrm{LSR}}$ & $\Delta v$ & \multirow{2}{*}{$\begin{array}{c}T_{\text {peak }} \\
(\mathrm{K})\end{array}$} & $V_{\mathrm{LSR}}$ & $\Delta v$ & \multirow{2}{*}{$\begin{array}{c}T_{\text {peak }} \\
(\mathrm{K})\end{array}$} & $V_{\mathrm{LSR}}$ & $\Delta v$ & \multirow{2}{*}{$\begin{array}{c}T_{\text {peak }} \\
(\mathrm{K})\end{array}$} & $V_{\mathrm{LSR}, \mathrm{GA}}$ & $\Delta v_{\mathrm{GA}}$ & $V_{\mathrm{LSR}, \mathrm{HFS}}$ & $\Delta v_{\mathrm{HFS}}$ & \multirow{3}{*}{$\begin{array}{l}T_{\mathrm{ex}} \\
(\mathrm{K}) \\
(16)\end{array}$} & \multirow{3}{*}{$\begin{array}{c}\tau \\
\\
(17)\end{array}$} & \multirow{3}{*}{$\begin{array}{c}T_{\text {ant }} \tau \\
(\mathrm{K}) \\
(18)\end{array}$} \\
\hline & & \multicolumn{2}{|c|}{$\left(\mathrm{km} \mathrm{s}^{-1}\right)$} & & \multicolumn{2}{|c|}{$\left(\mathrm{km} \mathrm{s}^{-1}\right)$} & & \multicolumn{2}{|c|}{$\left(\mathrm{km} \mathrm{s}^{-1}\right)$} & & & \multicolumn{2}{|c|}{$\left(\mathrm{km} \mathrm{s}^{-1}\right)$} & & & & \\
\hline (1) & $(2)$ & $(3)$ & $(4)$ & $(5)$ & $(6)$ & $(7)$ & $(8)$ & (9) & $(10)$ & $(11)$ & $(12)$ & (13) & $(14)$ & $(15)$ & & & \\
\hline G190.15-13.75North & $\leq 0.13$ & $\cdots$ & $\cdots$ & $\leq 0.20$ & $\cdots$ & $\cdots$ & $\leq 0.15$ & $\cdots$ & $\cdots$ & $\leq 0.21$ & $\cdots$ & $\cdots$ & $\cdots$ & $\cdots$ & $\cdots$ & $\cdots$ & $\cdots$ \\
\hline G190.15-13.75South & $\leq 0.13$ & .. & $\ldots$ & $\leq 0.18$ & $\cdots$ & $\ldots$ & $\leq 0.14$ & $\ldots$ & $\ldots$ & $\leq 0.18$ & $\cdots$ & ... & $\ldots$ & $\ldots$ & $\ldots$ & $\ldots$ & $\ldots$ \\
\hline G191.90-11.21North & $\leq 0.12$ & $\cdots$ & $\cdots$ & $\leq 0.16$ & $\cdots$ & $\cdots$ & $\leq 0.13$ & $\cdots$ & $\ldots$ & $\leq 0.14$ & $\cdots$ & $\cdots$ & $\cdots$ & $\cdots$ & $\cdots$ & $\cdots$ & $\cdots$ \\
\hline G191.90-11.21South & 0.38 & 10.49 & 0.49 & 0.44 & 10.59 & 1.00 & 0.11 & 10.52 & 0.76 & 0.16 & 10.73 & 0.26 & $\ldots$ & $\ldots$ & $7.4 \pm 3.7^{\dagger}$ & $\ldots$ & $\ldots$ \\
\hline G192.12-10.90North & 0.77 & 10.02 & 0.59 & 0.52 & 10.15 & 0.89 & 0.18 & 9.99 & 0.99 & 0.19 & 10.01 & 0.35 & $\ldots$ & $\ldots$ & $6.5 \pm 3.2^{\dagger}$ & $\ldots$ & $\ldots$ \\
\hline G192.12-10.90South & 0.21 & 10.11 & 0.54 & $\leq 0.16$ & $\ldots$ & $\ldots$ & $\leq 0.10$ & $\ldots$ & $\ldots$ & $\leq 0.13$ & $\ldots$ & $\ldots$ & $\ldots$ & $\ldots$ & $\ldots$ & $\ldots$ & $\ldots$ \\
\hline G192.12-11.10 & 0.37 & 10.12 & 0.85 & 0.33 & 10.32 & 1.35 & 0.13 & 10.43 & 1.09 & $\leq 0.13$ & $\cdots$ & $\cdots$ & $\cdots$ & $\cdots$ & $\cdots$ & $\cdots$ & $\cdots$ \\
\hline G192.32-11.88North & 0.47 & 12.14 & 0.64 & 0.69 & 12.13 & 1.02 & 0.23 & 12.31 & 0.60 & 0.18 & 12.02 & 0.68 & $\ldots$ & $\ldots$ & $8.7 \pm 4.3^{\dagger}$ & $\ldots$ & $\ldots$ \\
\hline G192.32-11.88South & 0.54 & 12.09 & 0.74 & 0.80 & 12.18 & 0.93 & 0.29 & 12.25 & 0.71 & 0.29 & 12.21 & 0.56 & 12.20 & 0.49 & $8.7 \pm 4.3^{\dagger}$ & $\cdots$ & $2.0 \pm 0.1$ \\
\hline G196.92-10.37 & 0.86 & 11.61 & 0.99 & 0.40 & 11.77 & 1.19 & 0.27 & 11.76 & 0.99 & $\leq 0.17$ & $\ldots$ & $\ldots$ & $\ldots$ & $\ldots$ & $\ldots$ & $\ldots$ & $\ldots$ \\
\hline G198.69-09.12North1 & 0.14 & 10.99 & 0.41 & 0.16 & 11.17 & 0.78 & $\leq 0.09$ & $\cdots$ & $\cdots$ & $\leq 0.11$ & $\cdots$ & $\cdots$ & $\cdots$ & $\cdots$ & $\cdots$ & $\cdots$ & $\cdots$ \\
\hline G198.69-09.12North2 & 0.73 & 10.67 & 0.61 & 0.41 & 10.74 & 1.00 & 0.16 & 10.77 & 0.70 & 0.11 & 10.80 & 0.42 & $\ldots$ & $\ldots$ & $5.7 \pm 2.9^{\dagger}$ & $\ldots$ & $\ldots$ \\
\hline G198.69-09.12South & 0.91 & 11.07 & 0.82 & 0.38 & 11.22 & 1.05 & 0.22 & 11.22 & 0.87 & $\leq 0.10$ & $\cdots$ & $\cdots$ & $\cdots$ & $\cdots$ & $\cdots$ & $\cdots$ & $\cdots$ \\
\hline G200.34-10.97North & 0.41 & 13.35 & 0.46 & 0.56 & 13.50 & 0.93 & 0.19 & 13.51 & 0.54 & 0.23 & 13.29 & 0.21 & $\ldots$ & $\ldots$ & $6.7 \pm 3.4^{\dagger}$ & $\ldots$ & $\ldots$ \\
\hline G200.34-10.97South & 0.48 & 13.58 & 0.57 & $\leq 0.26$ & $\cdots$ & $\cdots$ & $\leq 0.19$ & $\cdots$ & $\cdots$ & $\leq 0.22$ & $\cdots$ & $\cdots$ & $\cdots$ & $\cdots$ & $\cdots$ & $\cdots$ & $\cdots$ \\
\hline G201.52-11.08 & 0.22 & 9.20 & 0.45 & $\leq 0.29$ & $\ldots$ & $\ldots$ & $\leq 0.21$ & $\ldots$ & $\ldots$ & $\leq 0.26$ & $\ldots$ & $\ldots$ & $\ldots$ & $\ldots$ & $\ldots$ & $\ldots$ & $\ldots$ \\
\hline G201.72-11.22 & 1.05 & 9.51 & 0.32 & 0.59 & 9.53 & 0.81 & 0.35 & 9.56 & 0.47 & $\leq 0.24$ & $\ldots$ & $\cdots$ & $\cdots$ & $\cdots$ & $\cdots$ & $\cdots$ & $\cdots$ \\
\hline G203.21-11.20East1 & 0.74 & 9.94 & 0.97 & 1.11 & 10.58 & 1.21 & 0.41 & 10.57 & 0.95 & 0.37 & 10.66 & 0.45 & $\ldots$ & $\ldots$ & $5.6 \pm 2.8^{\dagger}$ & $\ldots$ & $\ldots$ \\
\hline G203.21-11.20East 2 & 0.70 & 10.13 & 0.74 & 1.05 & 10.24 & 1.03 & 0.31 & 10.38 & 0.82 & 0.40 & 10.32 & 0.40 & $\cdots$ & $\cdots$ & $5.6 \pm 2.8^{\dagger}$ & $\cdots$ & $\cdots$ \\
\hline G203.21-11.20West1 & 0.73 & 10.67 & 0.79 & 1.17 & 10.67 & 1.18 & 0.43 & 10.68 & 0.86 & 0.59 & 10.70 & 0.46 & 10.70 & 0.53 & $5.0 \pm 0.3$ & $2.2 \pm 1.4$ & $5.1 \pm 0.6$ \\
\hline G203.21-11.20West2 & 0.75 & 10.08 & 0.55 & 0.94 & 10.17 & 1.09 & 0.39 & 10.17 & 0.68 & 0.35 & 10.20 & 0.53 & 10.20 & 0.47 & $4.3 \pm 0.3$ & $2.1 \pm 2.2$ & $3.3 \pm 0.6$ \\
\hline G204.4-11.3A2East & 1.48 & 1.60 & 0.58 & 1.72 & 1.62 & 1.02 & 0.66 & 1.64 & 0.73 & 0.40 & 1.76 & 0.52 & 1.72 & 0.50 & $4.2 \pm 0.4$ & $2.5 \pm 2.9$ & $3.6 \pm 0.9$ \\
\hline G204.4-11.3A2West & 0.72 & 1.52 & 0.94 & 0.46 & 1.70 & 1.27 & $\leq 0.21$ & $\ldots$ & $\ldots$ & 0.24 & 1.26 & 0.34 & 1.22 & 0.53 & $5.6 \pm 2.8^{\dagger}$ & $\cdots$ & $0.8 \pm 0.3$ \\
\hline G205.46-14.56Middle1 & 0.79 & 9.99 & 0.87 & 0.33 & 10.07 & 1.19 & $\leq 0.17$ & $\ldots$ & $\ldots$ & $\leq 0.19$ & $\ldots$ & $\ldots$ & $\cdots$ & $\cdots$ & $\ldots$ & $\ldots$ & $\ldots$ \\
\hline G205.46-14.56Middle2 & 1.06 & 10.25 & 0.68 & 0.35 & 10.25 & 1.04 & 0.29 & 10.39 & 0.61 & $\leq 0.14$ & $\cdots$ & $\cdots$ & $\cdots$ & $\cdots$ & $\cdots$ & $\cdots$ & $\cdots$ \\
\hline G205.46-14.56Middle3 & 0.90 & 10.03 & 0.80 & 0.33 & 10.16 & 1.09 & 0.22 & 9.97 & 0.95 & $\leq 0.15$ & $\ldots$ & $\ldots$ & $\ldots$ & $\ldots$ & $\ldots$ & $\ldots$ & $\ldots$ \\
\hline G205.46-14.56North3 & 0.49 & 10.04 & 0.91 & 0.61 & 10.16 & 1.01 & 0.26 & 10.14 & 0.83 & 0.16 & 10.24 & 0.46 & $\ldots$ & $\ldots$ & $6.2 \pm 3.1^{\dagger}$ & $\ldots$ & $\ldots$ \\
\hline G205.46-14.56North2 & 0.65 & 9.79 & 0.89 & 0.38 & 10.14 & 1.03 & 0.16 & 10.12 & 0.98 & 0.14 & 10.20 & 0.27 & $\ldots$ & $\ldots$ & $6.2 \pm 3.1^{\dagger}$ & $\ldots$ & $\ldots$ \\
\hline G205.46-14.56North1 & 0.62 & 9.96 & 0.76 & 0.79 & 10.12 & 1.02 & 0.33 & 10.18 & 0.72 & 0.27 & 10.08 & 0.40 & $\ldots$ & $\cdots$ & $6.2 \pm 3.1^{\dagger}$ & $\cdots$ & $\ldots$ \\
\hline G205.46-14.56South1 & 0.33 & 10.33 & 1.29 & 0.28 & 10.19 & 1.50 & 0.27 & 10.44 & 0.97 & $\leq 0.14$ & $\cdots$ & ... & $\ldots$ & $\cdots$ & $\cdots$ & $\ldots$ & $\cdots$ \\
\hline G205.46-14.56South2 & 0.83 & 10.46 & 0.58 & 0.49 & 10.51 & 0.90 & 0.35 & 10.56 & 0.62 & $\leq 0.14$ & $\cdots$ & $\cdots$ & $\ldots$ & $\cdots$ & $\cdots$ & $\cdots$ & $\cdots$ \\
\hline G205.46-14.56South3 & 0.48 & 10.38 & 0.72 & 0.74 & 10.50 & 0.97 & 0.32 & 10.57 & 0.74 & 0.24 & 10.45 & 0.50 & 10.40 & 0.54 & $6.2 \pm 3.1^{\dagger}$ & $\cdots$ & $1.3 \pm 0.1$ \\
\hline G206.12-15.76 & 1.37 & 8.35 & 0.31 & 1.80 & 8.46 & 1.02 & 0.64 & 8.50 & 0.72 & 0.44 & 8.34 & 0.38 & 8.47 & 0.60 & $4.5 \pm 0.7$ & $1.8 \pm 4.4$ & $3.1 \pm 1.2$ \\
\hline G206.21-16.17North & $\leq 0.32$ & $\ldots$ & $\ldots$ & 0.56 & 9.89 & 0.79 & $\leq 0.37$ & $\ldots$ & $\ldots$ & 0.51 & 9.79 & 0.34 & $\ldots$ & $\ldots$ & $7.8 \pm 3.9^{\dagger}$ & $\ldots$ & $\ldots$ \\
\hline
\end{tabular}


Table 4 (continued)

\begin{tabular}{|c|c|c|c|c|c|c|c|c|c|c|c|c|c|c|c|c|c|}
\hline \multirow{3}{*}{ SCUBA-2 core } & \multicolumn{3}{|c|}{$\mathrm{c}-\mathrm{C}_{3} \mathrm{H}_{2}$} & \multicolumn{3}{|c|}{ DNC } & \multicolumn{3}{|c|}{$\mathrm{HN}^{13} \mathrm{C}$} & \multicolumn{8}{|c|}{$\mathrm{N}_{2} \mathrm{D}^{+}$} \\
\hline & \multirow{2}{*}{$\begin{array}{c}T_{\text {peak }} \\
(\mathrm{K})\end{array}$} & $V_{\mathrm{LSR}}$ & $\Delta v$ & \multirow{2}{*}{$\begin{array}{c}T_{\text {peak }} \\
(\mathrm{K})\end{array}$} & $V_{\mathrm{LSR}}$ & $\Delta v$ & \multirow{2}{*}{$\begin{array}{c}T_{\text {peak }} \\
(\mathrm{K})\end{array}$} & $V_{\mathrm{LSR}}$ & $\Delta v$ & \multirow{2}{*}{$\begin{array}{c}T_{\text {peak }} \\
(\mathrm{K})\end{array}$} & \multirow[t]{2}{*}{$V_{\mathrm{LSR}, \mathrm{GA}}$} & $\Delta v_{\mathrm{GA}}$ & $V_{\mathrm{LSR}, \mathrm{HFS}}$ & $\Delta v_{\mathrm{HFS}}$ & \multirow{3}{*}{$\begin{array}{l}T_{\text {ex }} \\
(\mathrm{K}) \\
(16)\end{array}$} & \multirow{3}{*}{$\begin{array}{c}\tau \\
(17)\end{array}$} & \multirow{3}{*}{$\begin{array}{r}T_{\text {ant }} \tau \\
(\mathrm{K}) \\
(18)\end{array}$} \\
\hline & & \multicolumn{2}{|c|}{$\left(\mathrm{km} \mathrm{s}^{-1}\right)$} & & \multicolumn{2}{|c|}{$\left(\mathrm{km} \mathrm{s}^{-1}\right)$} & & \multicolumn{2}{|c|}{$\left(\mathrm{km} \mathrm{s}^{-1}\right)$} & & & \multicolumn{2}{|c|}{$\left(\mathrm{km} \mathrm{s}^{-1}\right)$} & & & & \\
\hline$(1)$ & $(2)$ & $(3)$ & $(4)$ & $(5)$ & $(6)$ & $(7)$ & $(8)$ & $(9)$ & $(10)$ & $(11)$ & $(12)$ & $(13)$ & $(14)$ & $(15)$ & & & \\
\hline G206.69-16.60North & 0.39 & 11.27 & 0.33 & 0.52 & 11.49 & 0.85 & $\leq 0.22$ & $\cdots$ & $\cdots$ & $\leq 0.24$ & $\cdots$ & $\cdots$ & $\cdots$ & $\cdots$ & $\cdots$ & $\cdots$ & $\cdots$ \\
\hline G206.69-16.60South & 0.34 & 11.98 & 0.64 & 0.39 & 12.01 & 0.95 & $\leq 0.23$ & $\cdots$ & $\cdots$ & $\leq 0.22$ & $\cdots$ & $\cdots$ & $\cdots$ & $\cdots$ & $\cdots$ & $\cdots$ & $\cdots$ \\
\hline G206.93-16.61East 1 & 0.43 & 9.80 & 1.11 & 0.82 & 9.94 & 1.03 & 0.31 & 9.92 & 0.86 & $\leq 0.20$ & $\cdots$ & $\cdots$ & $\cdots$ & $\cdots$ & $\cdots$ & $\cdots$ & $\cdots$ \\
\hline G206.93-16.61East2 & 0.30 & 9.63 & 1.03 & 0.28 & 9.77 & 0.62 & 0.18 & 9.82 & 0.65 & $\leq 0.20$ & $\cdots$ & $\ldots$ & $\ldots$ & $\ldots$ & $\ldots$ & $\ldots$ & $\ldots$ \\
\hline G206.93-16.61West1 & 1.19 & 9.29 & 0.95 & 0.35 & 9.70 & 1.11 & 0.34 & 9.44 & 1.08 & $\leq 0.21$ & $\cdots$ & $\cdots$ & $\cdots$ & $\cdots$ & $\cdots$ & $\cdots$ & $\cdots$ \\
\hline G206.93-16.61West3 & 0.98 & 9.28 & 0.95 & 0.94 & 9.49 & 1.02 & 0.65 & 9.47 & 0.87 & 0.20 & 9.42 & 0.28 & .. & $\ldots$ & $8.4 \pm 4.2^{\dagger}$ & $\ldots$ & $\ldots$ \\
\hline G206.93-16.61West4 & 0.36 & 10.05 & 1.26 & 0.53 & 10.23 & 1.08 & 0.22 & 10.32 & 0.51 & 0.20 & 10.19 & 0.42 & 10.30 & 0.49 & $8.4 \pm 4.2^{\dagger}$ & $\ldots$ & $1.0 \pm 0.2$ \\
\hline G206.93-16.61West5 & 0.15 & 9.38 & 2.31 & 0.49 & 9.25 & 0.95 & $\leq 0.17$ & $\ldots$ & $\ldots$ & 0.30 & 9.05 & 0.25 & 9.11 & 0.40 & $8.4 \pm 4.2^{\dagger}$ & $\ldots$ & $1.8 \pm 0.1$ \\
\hline G206.93-16.61West6 & $\leq 0.14$ & $\cdots$ & $\cdots$ & $\leq 0.23$ & $\cdots$ & $\cdots$ & $\leq 0.18$ & $\cdots$ & $\cdots$ & $\leq 0.20$ & $\cdots$ & $\cdots$ & $\cdots$ & $\cdots$ & $\cdots$ & $\cdots$ & $\cdots$ \\
\hline G206.93-16.61West6 & 0.41 & 10.26 & 0.72 & 0.31 & 10.45 & 1.16 & 0.23 & 10.54 & 0.57 & $\leq 0.20$ & $\cdots$ & $\ldots$ & $\cdots$ & $\ldots$ & $\ldots$ & $\ldots$ & $\ldots$ \\
\hline G207.36-19.82North1 & 0.64 & 10.67 & 1.01 & 0.46 & 11.09 & 1.07 & 0.23 & 10.76 & 1.02 & $\leq 0.23$ & $\cdots$ & $\ldots$ & .. & $\cdots$ & $\ldots$ & $\ldots$ & $\ldots$ \\
\hline G207.36-19.82North2 & 0.55 & 11.10 & 0.56 & 1.07 & 11.36 & 0.90 & 0.24 & 11.25 & 0.68 & 0.34 & 11.32 & 0.54 & 11.30 & 0.43 & $5.9 \pm 3.0^{\dagger}$ & $\ldots$ & $2.7 \pm 0.5$ \\
\hline G207.36-19.82North3 & 0.41 & 11.00 & 0.55 & 0.68 & 11.30 & 0.98 & $\leq 0.20$ & $\ldots$ & $\ldots$ & $\leq 0.21$ & $\cdots$ & $\ldots$ & $\ldots$ & $\ldots$ & $\ldots$ & $\ldots$ & $\ldots$ \\
\hline G207.36-19.82North4 & 0.43 & 10.91 & 0.47 & 0.40 & 11.29 & 1.33 & $\leq 0.19$ & $\ldots$ & $\ldots$ & $\leq 0.21$ & $\cdots$ & $\cdots$ & $\cdots$ & $\cdots$ & $\ldots$ & $\ldots$ & $\ldots$ \\
\hline G207.36-19.82South & 0.65 & 11.17 & 0.51 & 0.46 & 11.45 & 1.11 & $\leq 0.19$ & $\ldots$ & $\ldots$ & 0.23 & 11.41 & 0.20 & 11.40 & 0.38 & $5.9 \pm 3.0^{\dagger}$ & $\ldots$ & $1.0 \pm 0.3$ \\
\hline G207.3-19.8A2North1 & $\leq 0.17$ & $\ldots$ & $\ldots$ & $\leq 0.24$ & $\ldots$ & $\ldots$ & $\leq 0.19$ & $\cdots$ & $\ldots$ & $\leq 0.22$ & $\cdots$ & $\ldots$ & $\ldots$ & $\ldots$ & $\ldots$ & $\ldots$ & $\ldots$ \\
\hline G207.3-19.8A2North2 & 0.22 & 12.51 & 0.37 & 0.28 & 12.79 & 0.79 & $\leq 0.18$ & $\cdots$ & $\cdots$ & $\leq 0.23$ & $\cdots$ & $\cdots$ & $\cdots$ & $\cdots$ & $\cdots$ & $\cdots$ & $\cdots$ \\
\hline G207.3-19.8A2North3 & $\leq 0.14$ & $\ldots$ & $\ldots$ & $\leq 0.26$ & $\cdots$ & $\ldots$ & $\leq 0.18$ & $\ldots$ & $\cdots$ & $\leq 0.22$ & $\ldots$ & $\ldots$ & .. & $\ldots$ & $\ldots$ & $\ldots$ & $\cdots$ \\
\hline G207.3-19.8A2South & $\leq 0.20$ & $\cdots$ & $\cdots$ & $\leq 0.32$ & $\cdots$ & $\cdots$ & $\leq 0.24$ & $\cdots$ & $\cdots$ & $\leq 0.29$ & $\cdots$ & $\cdots$ & $\cdots$ & $\cdots$ & $\cdots$ & $\cdots$ & $\cdots$ \\
\hline G208.68-19.20North1 & 0.62 & 11.09 & 1.17 & 0.80 & 11.26 & 1.15 & 0.45 & 11.28 & 0.83 & $\leq 0.20$ & $\ldots$ & $\ldots$ & $\ldots$ & $\ldots$ & $\ldots$ & $\ldots$ & $\ldots$ \\
\hline G208.68-19.20North2 & 0.50 & 11.12 & 1.16 & 1.44 & 11.27 & 0.95 & 0.63 & 11.28 & 0.75 & 0.82 & 11.21 & 0.49 & 11.20 & 0.44 & $4.9 \pm 0.2$ & $4.3 \pm 1.2$ & $9.5 \pm 0.9$ \\
\hline G208.68-19.20North3 & 0.47 & 10.69 & 1.71 & 1.02 & 11.25 & 1.07 & 0.36 & 11.32 & 1.23 & 0.45 & 11.17 & 0.49 & 11.10 & 0.45 & $4.6 \pm 0.4$ & $2.3 \pm 2.3$ & $4.2 \pm 0.8$ \\
\hline G208.68-19.20South & 0.33 & 10.39 & 1.68 & 0.46 & 10.44 & 1.45 & 0.22 & 10.42 & 0.95 & 0.26 & 10.37 & 0.43 & $\ldots$ & $\ldots$ & $9.8 \pm 4.9^{\dagger}$ & $\ldots$ & $\cdots$ \\
\hline G208.89-20.04East & 1.74 & 8.72 & 0.51 & 1.21 & 8.88 & 0.90 & 0.73 & 8.88 & 0.58 & 0.38 & 8.82 & 0.42 & $\ldots$ & $\ldots$ & $6.4 \pm 3.2^{\dagger}$ & $\ldots$ & $\ldots$ \\
\hline G209.05-19.73North & 0.52 & 8.17 & 0.52 & 0.40 & 8.41 & 0.96 & 0.21 & 8.32 & 0.31 & 0.27 & 8.09 & 0.19 & 8.32 & 0.54 & $7.8 \pm 3.9^{\dagger}$ & $\ldots$ & $1.0 \pm 0.2$ \\
\hline G209.05-19.73South & 0.81 & 7.79 & 0.38 & 0.65 & 7.95 & 0.94 & 0.21 & 7.95 & 0.52 & 0.26 & 8.04 & 0.23 & $\ldots$ & $\ldots$ & $7.8 \pm 3.9^{\dagger}$ & $\ldots$ & $\ldots$ \\
\hline G209.29-19.65North1 & $\leq 0.24$ & $\cdots$ & $\cdots$ & 0.30 & 8.51 & 0.98 & 0.25 & 8.54 & 0.57 & 0.29 & 8.50 & 0.34 & 8.52 & 0.29 & $6.3 \pm 0.5$ & $0.5 \pm 0.1$ & $1.8 \pm 0.2$ \\
\hline G209.29-19.65North2 & $\leq 0.18$ & $\ldots$ & $\ldots$ & $\leq 0.19$ & $\ldots$ & $\ldots$ & $\leq 0.15$ & $\ldots$ & $\ldots$ & $\leq 0.16$ & $\ldots$ & $\ldots$ & $\ldots$ & $\ldots$ & $\ldots$ & $\ldots$ & $\ldots$ \\
\hline G209.29-19.65North2 & $\leq 0.18$ & $\ldots$ & $\cdots$ & 0.38 & 8.86 & 0.97 & $\leq 0.15$ & $\cdots$ & $\cdots$ & 0.16 & 8.77 & 0.48 & 8.77 & 0.52 & $8.6 \pm 4.3^{\dagger}$ & $\cdots$ & $1.0 \pm 0.2$ \\
\hline G209.29-19.65North3 & $\leq 0.17$ & $\ldots$ & $\ldots$ & $\leq 0.18$ & $\ldots$ & $\ldots$ & 0.16 & 8.62 & 0.84 & $\leq 0.17$ & $\cdots$ & $\ldots$ & $\ldots$ & $\ldots$ & $\ldots$ & $\ldots$ & $\ldots$ \\
\hline G209.29-19.65South1 & $\leq 0.20$ & $\ldots$ & $\ldots$ & 0.51 & 7.50 & 1.55 & $\leq 0.18$ & $\ldots$ & $\ldots$ & 0.20 & 7.20 & 0.97 & 7.34 & 0.95 & $8.6 \pm 4.3^{\dagger}$ & $\ldots$ & $1.1 \pm 0.2$ \\
\hline G209.29-19.65South2 & 0.16 & 8.32 & 1.13 & 0.44 & 7.75 & 1.49 & 0.20 & 7.77 & 0.95 & $\leq 0.17$ & $\ldots$ & $\ldots$ & $\ldots$ & $\ldots$ & $\ldots$ & $\ldots$ & $\ldots$ \\
\hline G209.29-19.65South2 & $\leq 0.16$ & $\cdots$ & $\cdots$ & 0.26 & 9.03 & 0.48 & 0.20 & 8.96 & 0.87 & 0.21 & 8.85 & 0.48 & $\ldots$ & $\cdots$ & $8.6 \pm 4.3^{\dagger}$ & $\cdots$ & $\cdots$ \\
\hline G209.29-19.65South3 & $\leq 0.19$ & $\ldots$ & $\ldots$ & 0.39 & 7.57 & 0.74 & $\leq 0.16$ & $\ldots$ & $\ldots$ & $\leq 0.16$ & $\ldots$ & $\ldots$ & $\ldots$ & $\ldots$ & $\ldots$ & $\ldots$ & $\ldots$ \\
\hline G209.55-19.68North1 & 0.35 & 7.45 & 1.10 & 0.50 & 7.25 & 1.02 & 0.34 & 7.41 & 0.75 & $\leq 0.16$ & $\cdots$ & $\cdots$ & $\cdots$ & $\cdots$ & $\ldots$ & $\cdots$ & $\cdots$ \\
\hline G209.55-19.68North2 & 0.30 & 8.30 & 0.35 & 0.48 & 8.16 & 0.96 & 0.31 & 8.34 & 0.37 & 0.23 & 8.14 & 0.32 & $\ldots$ & $\ldots$ & $7.0 \pm 3.5^{\dagger}$ & $\ldots$ & $\ldots$ \\
\hline
\end{tabular}


Table 4 (continued)

\begin{tabular}{|c|c|c|c|c|c|c|c|c|c|c|c|c|c|c|c|c|c|}
\hline \multirow{3}{*}{ SCUBA-2 core } & \multicolumn{3}{|c|}{ c- $\mathrm{C}_{3} \mathrm{H}_{2}$} & \multicolumn{3}{|c|}{$\mathrm{DNC}$} & \multicolumn{3}{|c|}{$\mathrm{HN}^{13} \mathrm{C}$} & \multicolumn{8}{|c|}{$\mathrm{N}_{2} \mathrm{D}^{+}$} \\
\hline & \multirow{2}{*}{$\begin{array}{c}T_{\text {peak }} \\
(\mathrm{K})\end{array}$} & $V_{\mathrm{LSR}}$ & $\Delta v$ & \multirow{2}{*}{$\begin{array}{c}T_{\text {peak }} \\
(\mathrm{K})\end{array}$} & $V_{\mathrm{LSR}}$ & $\Delta v$ & \multirow{2}{*}{$\begin{array}{c}T_{\text {peak }} \\
(\mathrm{K})\end{array}$} & $V_{\mathrm{LSR}}$ & $\Delta v$ & \multirow{2}{*}{$\begin{array}{c}T_{\text {peak }} \\
(\mathrm{K})\end{array}$} & $V_{\mathrm{LSR}, \mathrm{GA}}$ & $\Delta v_{\mathrm{GA}}$ & $V_{\mathrm{LSR}, \mathrm{HFS}}$ & $\Delta v_{\mathrm{HFS}}$ & \multirow{3}{*}{$\begin{array}{l}T_{\text {ex }} \\
(\mathrm{K}) \\
(16)\end{array}$} & \multirow[t]{2}{*}{$\tau$} & \multirow{2}{*}{$\begin{array}{r}T_{\text {ant }} \tau \\
(\mathrm{K})\end{array}$} \\
\hline & & \multicolumn{2}{|c|}{$\left(\mathrm{km} \mathrm{s}^{-1}\right)$} & & \multicolumn{2}{|c|}{$\left(\mathrm{km} \mathrm{s}^{-1}\right)$} & & \multicolumn{2}{|c|}{$\left(\mathrm{km} \mathrm{s}^{-1}\right)$} & & & \multicolumn{2}{|c|}{$\left(\mathrm{km} \mathrm{s}^{-1}\right)$} & & & & \\
\hline (1) & (2) & (3) & (4) & $(5)$ & (6) & (7) & (8) & (9) & $(10)$ & (11) & (12) & (13) & (14) & $(15)$ & & (17) & (18) \\
\hline G209.55-19.68North3 & 0.75 & 8.15 & 0.58 & 0.58 & 8.01 & 1.19 & 0.31 & 8.14 & 0.92 & 0.19 & 7.91 & 0.32 & 7.88 & 0.57 & $7.0 \pm 3.5^{\dagger}$ & $\cdots$ & $1.0 \pm 0.2$ \\
\hline G209.55-19.68South1 & 0.43 & 7.43 & 0.75 & 0.74 & 7.43 & 0.89 & 0.35 & 7.53 & 0.64 & 0.24 & 7.50 & 0.41 & 7.41 & 0.52 & $7.0 \pm 3.5^{\dagger}$ & $\cdots$ & $1.2 \pm 0.2$ \\
\hline G209.55-19.68South3 & 0.84 & 8.14 & 0.57 & 0.84 & 8.13 & 0.93 & 0.45 & 8.22 & 0.64 & 0.26 & 8.06 & 0.47 & 8.04 & 0.47 & $7.0 \pm 3.5^{\dagger}$ & $\cdots$ & $1.6 \pm 0.2$ \\
\hline G209.77-19.40East1 & 0.67 & 8.12 & 0.55 & 0.72 & 8.00 & 0.84 & 0.45 & 8.13 & 0.74 & 0.25 & 8.00 & 0.26 & 7.96 & 0.47 & $7.0 \pm 3.5^{\dagger}$ & $\cdots$ & $1.2 \pm 0.2$ \\
\hline G209.77-19.40East2 & 0.67 & 8.08 & 0.60 & 0.77 & 8.07 & 0.88 & 0.34 & 8.08 & 0.85 & $\leq 0.20$ & $\cdots$ & $\cdots$ & $\cdots$ & $\cdots$ & $\cdots$ & $\cdots$ & .. \\
\hline G209.77-19.40East3 & 0.55 & 7.87 & 0.27 & 0.73 & 7.86 & 0.89 & 0.20 & 7.87 & 0.77 & 0.21 & 7.65 & 0.42 & 7.71 & 0.33 & $7.0 \pm 3.5^{\dagger}$ & $\cdots$ & $1.8 \pm 0.2$ \\
\hline G209.77-19.40East3 & 0.46 & 8.38 & 0.28 & 0.49 & 8.50 & 0.32 & 0.21 & 8.31 & 0.41 & $\leq 0.17$ & $\cdots$ & $\cdots$ & $\cdots$ & $\cdots$ & $\cdots$ & $\cdots$ & $\cdots$ \\
\hline G209.77-19.40West & 0.57 & 8.40 & 0.22 & 0.35 & 8.31 & 0.75 & 0.27 & 8.38 & 0.46 & $\leq 0.18$ & $\cdots$ & $\cdots$ & $\cdots$ & $\cdots$ & $\cdots$ & $\cdots$ & $\cdots$ \\
\hline G209.77-19.61East & 0.44 & 8.06 & 0.31 & 0.26 & 7.66 & 0.21 & $\leq 0.18$ & $\cdots$ & $\cdots$ & $\leq 0.19$ & $\cdots$ & $\cdots$ & $\cdots$ & $\cdots$ & $\cdots$ & $\cdots$ & $\cdots$ \\
\hline G209.77-19.61West & 0.31 & 7.28 & 0.36 & 0.45 & 7.18 & 0.93 & $\leq 0.18$ & $\cdots$ & $\cdots$ & $\leq 0.17$ & $\cdots$ & $\cdots$ & $\cdots$ & $\cdots$ & $\cdots$ & $\cdots$ & $\cdots$ \\
\hline G209.79-19.80East & 0.86 & 5.57 & 0.37 & 0.21 & 5.36 & 0.75 & $\leq 0.17$ & $\cdots$ & $\cdots$ & $\leq 0.18$ & $\cdots$ & $\cdots$ & $\cdots$ & $\cdots$ & $\cdots$ & $\cdots$ & $\cdots$ \\
\hline G209.79-19.80West & 0.86 & 5.83 & 0.70 & 0.84 & 5.77 & 1.12 & 0.38 & 5.86 & 0.71 & 0.25 & 5.72 & 0.39 & 5.69 & 0.42 & $6.9 \pm 3.5^{\dagger}$ & $\cdots$ & $1.4 \pm 0.2$ \\
\hline G209.79-19.80West & 0.44 & 7.05 & 0.35 & $\leq 0.17$ & .. & $\cdots$ & $\leq 0.17$ & $\ldots$ & $\cdots$ & $\leq 0.18$ & $\cdots$ & $\cdots$ & $\ldots$ & $\cdots$ & $\cdots$ & $\cdots$ & .. \\
\hline G209.94-19.52North & 0.89 & 7.99 & 0.80 & 1.50 & 7.95 & 1.12 & 0.68 & 8.05 & 0.79 & 0.39 & 7.97 & 0.57 & 7.97 & 0.56 & $4.0 \pm 0.2$ & $3.2 \pm 2.2$ & $4.0 \pm 0.8$ \\
\hline G209.94-19.52South1 & 0.74 & 7.63 & 0.39 & 1.18 & 7.92 & 1.19 & 0.49 & 7.60 & 0.49 & 0.23 & 7.41 & 0.55 & $\cdots$ & $\cdots$ & $8.1 \pm 4.0^{\dagger}$ & $\cdots$ & $\cdots$ \\
\hline G209.94-19.52South1 & 0.86 & 8.20 & 0.43 & 1.24 & 8.14 & 0.83 & 0.55 & 8.15 & 0.51 & 0.37 & 8.05 & 0.44 & 8.06 & 0.41 & $8.1 \pm 4.0^{\dagger}$ & $\cdots$ & $2.4 \pm 0.2$ \\
\hline G209.94-19.52South2 & 0.68 & 7.52 & 0.67 & 0.46 & 7.38 & 1.23 & 0.21 & 7.53 & 1.01 & $\leq 0.17$ & $\cdots$ & $\cdots$ & $\cdots$ & $\cdots$ & $\cdots$ & $\cdots$ & $\cdots$ \\
\hline G210.37-19.53North & 0.56 & 6.37 & 0.32 & 0.61 & 6.49 & 0.79 & 0.19 & 6.42 & 0.35 & $\leq 0.19$ & $\cdots$ & $\cdots$ & $\cdots$ & $\cdots$ & $\cdots$ & $\cdots$ & $\cdots$ \\
\hline G210.37-19.53South & 0.79 & 5.82 & 1.02 & 0.66 & 5.99 & 1.22 & 0.26 & 5.96 & 1.26 & 0.27 & 5.47 & 0.28 & 5.57 & 0.47 & $7.0 \pm 3.5^{\dagger}$ & $\cdots$ & $1.2 \pm 0.2$ \\
\hline G210.49-19.79East1 & 0.96 & 8.59 & 0.88 & 0.57 & 8.66 & 1.09 & 0.27 & 8.85 & 1.09 & $\leq 0.20$ & $\cdots$ & $\cdots$ & $\cdots$ & $\cdots$ & $\cdots$ & $\cdots$ & $\cdots$ \\
\hline G210.49-19.79East2 & 0.38 & 7.90 & 0.43 & 0.67 & 8.13 & 0.73 & 0.27 & 8.10 & 0.49 & 0.27 & 8.09 & 0.25 & 8.08 & 0.33 & $5.9 \pm 3.0^{\dagger}$ & $\cdots$ & $1.7 \pm 0.2$ \\
\hline G210.49-19.79East2 & 1.36 & 10.30 & 0.73 & 0.53 & 10.47 & 1.15 & 0.34 & 10.42 & 0.94 & $\leq 0.19$ & $\cdots$ & $\cdots$ & $\cdots$ & $\cdots$ & $\cdots$ & $\cdots$ & $\cdots$ \\
\hline G210.49-19.79West & 0.64 & 9.08 & 0.97 & 0.89 & 9.31 & 1.18 & 0.44 & 9.16 & 0.85 & 0.24 & 9.03 & 1.03 & 9.24 & 0.67 & $5.9 \pm 3.0^{\dagger}$ & $\cdots$ & $1.8 \pm 0.2$ \\
\hline G210.82-19.47North1 & 0.47 & 5.31 & 0.76 & 0.82 & 5.43 & 0.98 & 0.21 & 5.47 & 1.12 & 0.45 & 5.33 & 0.49 & 5.34 & 0.41 & $8.1 \pm 4.0^{\dagger}$ & $\cdots$ & $3.4 \pm 0.2$ \\
\hline G210.82-19.47North2 & 0.74 & 5.15 & 0.39 & 1.00 & 5.34 & 0.92 & 0.29 & 5.36 & 0.67 & 0.37 & 5.25 & 0.25 & 5.24 & 0.28 & $8.1 \pm 4.0^{\dagger}$ & $\cdots$ & $2.5 \pm 0.2$ \\
\hline G210.97-19.33North & 0.16 & 3.78 & 0.69 & $\leq 0.16$ & $\cdots$ & $\cdots$ & $\leq 0.17$ & $\cdots$ & $\cdots$ & $\leq 0.17$ & $\cdots$ & $\cdots$ & $\ldots$ & $\cdots$ & $\cdots$ & $\cdots$ & $\cdots$ \\
\hline G210.97-19.33South1 & 1.12 & 2.87 & 0.41 & $\leq 0.38$ & $\cdots$ & $\cdots$ & $\leq 0.30$ & $\cdots$ & $\cdots$ & $\leq 0.36$ & $\cdots$ & $\cdots$ & $\cdots$ & $\cdots$ & $\cdots$ & $\cdots$ & $\cdots$ \\
\hline G210.97-19.33South2 & 0.69 & 3.27 & 0.89 & 0.49 & 3.34 & 0.27 & 0.40 & 3.10 & 0.27 & $\leq 0.34$ & $\cdots$ & $\cdots$ & $\cdots$ & $\cdots$ & $\cdots$ & $\cdots$ & $\cdots$ \\
\hline G210.97-19.33South2 & 0.80 & 4.54 & 0.43 & 0.89 & 4.47 & 0.75 & $\leq 0.33$ & $\ldots$ & $\ldots$ & $\leq 0.34$ & $\cdots$ & $\ldots$ & $\ldots$ & $\cdots$ & & $\cdots$ & $\cdots$ \\
\hline G211.01-19.54North & 1.15 & 5.96 & 1.01 & 0.87 & 5.96 & 1.33 & 0.46 & 5.98 & 0.95 & 0.27 & 5.79 & 1.41 & $\cdots$ & $\cdots$ & $7.3 \pm 3.6^{\dagger}$ & $\cdots$ & $\cdots$ \\
\hline G211.01-19.54South & 0.90 & 5.78 & 0.78 & 0.75 & 5.82 & 1.13 & 0.52 & 5.85 & 0.78 & 0.25 & 5.66 & 0.74 & 5.79 & 0.78 & $4.4 \pm 0.5$ & $1.1 \pm 2.7$ & $1.8 \pm 0.5$ \\
\hline G211.16-19.33North1 & 0.93 & 3.25 & 0.58 & 0.35 & 3.52 & 0.84 & 0.29 & 3.44 & 0.68 & $\leq 0.15$ & $\cdots$ & $\cdots$ & $\cdots$ & $\cdots$ & $\cdots$ & $\cdots$ & $\cdots$ \\
\hline G211.16-19.33North2 & 1.09 & 3.35 & 0.63 & 0.75 & 3.57 & 1.11 & 0.39 & 3.48 & 0.85 & 0.23 & 3.63 & 0.41 & 3.56 & 0.38 & $7.1 \pm 1.8$ & $0.4 \pm 12.1$ & $1.6 \pm 0.7$ \\
\hline G211.16-19.33North3 & 1.34 & 3.25 & 0.43 & 0.96 & 3.48 & 1.09 & 0.46 & 3.36 & 0.68 & 0.37 & 3.38 & 0.54 & 3.38 & 0.45 & $5.6 \pm 0.6$ & $1.1 \pm 2.5$ & $3.0 \pm 0.6$ \\
\hline G211.16-19.33North4 & 0.83 & 4.49 & 0.86 & 0.69 & 4.54 & 1.23 & 0.28 & 4.57 & 1.39 & 0.22 & 4.47 & 0.59 & 4.52 & 0.35 & $4.7 \pm 0.6$ & $0.9 \pm 3.4$ & $1.9 \pm 0.6$ \\
\hline G211.16-19.33North5 & 0.84 & 4.19 & 0.76 & 1.06 & 4.36 & 1.01 & 0.50 & 4.26 & 0.69 & 0.42 & 4.40 & 0.43 & 4.37 & 0.37 & $4.3 \pm 0.3$ & $2.6 \pm 2.0$ & $4.0 \pm 0.7$ \\
\hline
\end{tabular}

Table 4 continued 
Table 4 (continued)

\begin{tabular}{|c|c|c|c|c|c|c|c|c|c|c|c|c|c|c|c|c|c|}
\hline \multirow{3}{*}{ SCUBA-2 core } & \multicolumn{3}{|c|}{ c- $\mathrm{C}_{3} \mathrm{H}_{2}$} & \multicolumn{3}{|c|}{$\mathrm{DNC}$} & \multicolumn{3}{|c|}{$\mathrm{HN}^{13} \mathrm{C}$} & \multicolumn{8}{|c|}{$\mathrm{N}_{2} \mathrm{D}^{+}$} \\
\hline & \multirow{2}{*}{$\begin{array}{c}T_{\text {peak }} \\
(\mathrm{K})\end{array}$} & $V_{\mathrm{LSR}}$ & $\Delta v$ & \multirow{2}{*}{$\begin{array}{c}T_{\text {peak }} \\
(\mathrm{K})\end{array}$} & $V_{\mathrm{LSR}}$ & $\Delta v$ & \multirow{2}{*}{$\begin{array}{c}T_{\text {peak }} \\
(\mathrm{K})\end{array}$} & $V_{\mathrm{LSR}}$ & $\Delta v$ & \multirow{2}{*}{$\begin{array}{c}T_{\text {peak }} \\
(\mathrm{K})\end{array}$} & \multirow[t]{2}{*}{$V_{\mathrm{LSR}, \mathrm{GA}}$} & $\Delta v_{\mathrm{GA}}$ & $V_{\mathrm{LSR}, \mathrm{HFS}}$ & $\Delta v_{\mathrm{HFS}}$ & \multirow{3}{*}{$\begin{array}{l}T_{\text {ex }} \\
(\mathrm{K}) \\
(16)\end{array}$} & \multirow{3}{*}{$\begin{array}{c}\tau \\
(17) \\
\end{array}$} & \multirow{3}{*}{$\begin{array}{c}T_{\text {ant }} \tau \\
(\mathrm{K}) \\
(18)\end{array}$} \\
\hline & & \multicolumn{2}{|c|}{$\left(\mathrm{km} \mathrm{s}^{-1}\right)$} & & \multicolumn{2}{|c|}{$\left(\mathrm{km} \mathrm{s}^{-1}\right)$} & & \multicolumn{2}{|c|}{$\left(\mathrm{km} \mathrm{s}^{-1}\right)$} & & & \multicolumn{2}{|c|}{$\left(\mathrm{km} \mathrm{s}^{-1}\right)$} & & & & \\
\hline (1) & (2) & (3) & $(4)$ & $(5)$ & (6) & (7) & $(8)$ & $(9)$ & $(10)$ & $(11)$ & $(12)$ & $(13)$ & $(14)$ & $(15)$ & & & \\
\hline G211.16-19.33South & 0.24 & 3.19 & 0.53 & $\leq 0.18$ & $\cdots$ & & $\leq 0.16$ & $\cdots$ & $\cdots$ & $\leq 0.17$ & & & $\cdots$ & $\cdots$ & $\cdots$ & $\cdots$ & $\cdots$ \\
\hline G211.47-19.27North & 0.63 & 3.96 & 0.72 & 0.91 & 4.21 & 1.18 & 0.48 & 4.17 & 0.72 & 0.27 & 4.18 & 0.46 & 4.18 & 0.56 & $6.2 \pm 3.1^{\dagger}$ & $\cdots$ & $2.0 \pm 0.4$ \\
\hline G211.47-19.27South & 0.36 & 5.12 & 1.49 & 0.60 & 5.26 & 1.34 & 0.34 & 5.39 & 1.03 & $\leq 0.17$ & $\cdots$ & $\cdots$ & $\cdots$ & $\cdots$ & $\cdots$ & $\cdots$ & $\cdots$ \\
\hline G211.72-19.25North & 0.74 & 3.50 & 0.55 & 0.75 & 3.55 & 0.99 & 0.31 & 3.54 & 0.52 & $\leq 0.17$ & $\cdots$ & $\ldots$ & $\ldots$ & $\ldots$ & $\cdots$ & $\cdots$ & $\ldots$ \\
\hline G211.72-19.25South1 & 0.44 & 4.49 & 0.69 & 0.45 & 4.30 & 1.00 & 0.35 & 4.32 & 0.43 & $\leq 0.15$ & $\cdots$ & $\cdots$ & $\cdots$ & $\cdots$ & $\cdots$ & $\cdots$ & $\cdots$ \\
\hline G212.10-19.15North1 & 0.48 & 4.21 & 0.66 & 1.34 & 4.49 & 1.28 & 0.30 & 4.54 & 1.18 & 0.41 & 4.67 & 0.55 & 4.52 & 0.82 & $6.0 \pm 0.5$ & $0.9 \pm 1.5$ & $2.9 \pm 0.5$ \\
\hline G212.10-19.15North2 & 0.38 & 4.38 & 1.00 & 0.54 & 4.54 & 1.15 & 0.33 & 4.54 & 0.86 & 0.18 & 4.41 & 0.81 & $\ldots$ & $\ldots$ & $5.4 \pm 2.7^{\dagger}$ & $\ldots$ & $\ldots$ \\
\hline G212.10-19.15North3 & 1.07 & 4.07 & 0.76 & 0.80 & 4.34 & 1.27 & 0.44 & 4.38 & 0.86 & 0.21 & 4.45 & 0.33 & $\cdots$ & $\ldots$ & $6.8 \pm 3.4^{\dagger}$ & .. & $\ldots$ \\
\hline G212.10-19.15South & 0.60 & 3.90 & 0.74 & 0.87 & 3.98 & 1.06 & 0.34 & 3.98 & 0.83 & 0.31 & 3.82 & 0.56 & 3.91 & 0.72 & $5.4 \pm 2.7^{\dagger}$ & $\ldots$ & $2.1 \pm 0.2$ \\
\hline G212.84-19.45North & 0.83 & 4.32 & 0.39 & 0.79 & 4.34 & 0.87 & 0.44 & 4.39 & 0.61 & 0.28 & 4.30 & 0.38 & 4.38 & 0.35 & $5.9 \pm 3.0^{\dagger}$ & $\ldots$ & $1.7 \pm 0.2$ \\
\hline G212.84-19.45South & 0.36 & 4.48 & 0.33 & $\leq 0.21$ & $\cdots$ & $\cdots$ & $\leq 0.17$ & $\cdots$ & $\cdots$ & $\leq 0.19$ & $\cdots$ & $\cdots$ & $\cdots$ & $\cdots$ & $\cdots$ & $\cdots$ & $\cdots$ \\
\hline G215.44-16.38 & 1.22 & 11.21 & 0.42 & 0.99 & 11.46 & 0.89 & 0.44 & 11.47 & 0.62 & $\leq 0.18$ & $\ldots$ & .. & $\cdots$ & . & $\cdots$ & $\cdots$ & $\ldots$ \\
\hline G215.87-17.62North & 0.64 & 9.31 & 0.22 & $\leq 0.21$ & $\cdots$ & $\cdots$ & $\leq 0.20$ & $\cdots$ & $\cdots$ & $\leq 0.21$ & $\cdots$ & $\cdots$ & $\cdots$ & $\cdots$ & $\cdots$ & $\cdots$ & $\cdots$ \\
\hline G215.87-17.62Middle & 0.98 & 8.95 & 0.36 & 0.62 & 9.07 & 0.94 & 0.32 & 9.10 & 0.63 & 0.25 & 8.96 & 0.37 & 9.00 & 0.38 & $6.1 \pm 3.0^{\dagger}$ & $\ldots$ & $1.7 \pm 0.2$ \\
\hline G215.87-17.62South & 0.74 & 9.90 & 0.64 & 0.66 & 10.01 & 1.04 & 0.34 & 10.01 & 0.83 & 0.17 & 10.01 & 0.50 & 10.10 & 0.80 & $6.1 \pm 3.0^{\dagger}$ & $\ldots$ & $0.7 \pm 0.2$ \\
\hline SCOPEG139.12-03.23 & 0.12 & -31.72 & 1.85 & $\leq 0.11$ & $\ldots$ & $\ldots$ & $\leq 0.09$ & $\ldots$ & $\ldots$ & $\leq 0.10$ & $\cdots$ & . & $\ldots$ & $\ldots$ & $\ldots$ & $\ldots$ & $\cdots$ \\
\hline SCOPEG159.21-20.13 & 0.91 & 6.33 & 0.95 & 1.44 & 6.44 & 1.18 & 0.69 & 6.39 & 0.89 & 0.66 & 6.44 & 0.61 & 6.47 & 0.51 & $5.7 \pm 0.2$ & $2.2 \pm 0.7$ & $6.5 \pm 0.4$ \\
\hline SCOPEG159.18-20.09 & 1.02 & 6.23 & 0.88 & 1.90 & 6.18 & 1.23 & 0.85 & 6.17 & 0.87 & 0.50 & 6.02 & 0.83 & 6.11 & 0.82 & $6.5 \pm 3.2^{\dagger}$ & $\ldots$ & $3.2 \pm 0.1$ \\
\hline SCOPEG159.22-20.11 & 1.05 & 6.55 & 1.36 & 2.58 & 6.82 & 1.57 & 0.93 & 6.77 & 1.19 & 1.19 & 6.65 & 0.94 & 6.71 & 0.83 & $9.8 \pm 0.2$ & $1.4 \pm 0.2$ & $9.5 \pm 0.3$ \\
\hline SCOPEG162.46-08.67 & 0.55 & -1.28 & 1.00 & 0.34 & -1.23 & 1.04 & 0.22 & -1.35 & 0.58 & 0.15 & -1.39 & 0.33 & $\cdots$ & $\ldots$ & $6.1 \pm 3.0^{\dagger}$ & $\ldots$ & $\ldots$ \\
\hline SCOPEG162.48-08.68 & 0.51 & -1.37 & 0.76 & 0.44 & -1.29 & 0.89 & 0.23 & -1.39 & 0.56 & 0.17 & -1.37 & 0.45 & $\ldots$ & $\ldots$ & $6.1 \pm 3.0^{\dagger}$ & $\ldots$ & $\ldots$ \\
\hline SCOPEG171.50-14.91 & 2.34 & 6.28 & 0.39 & 1.23 & 6.52 & 1.14 & 0.74 & 6.46 & 0.69 & 0.50 & 6.54 & 0.37 & 6.55 & 0.34 & $4.5 \pm 0.2$ & $2.8 \pm 1.3$ & $4.9 \pm 0.5$ \\
\hline SCOPEG172.88+02.26 & 0.92 & -17.39 & 1.36 & 0.52 & -17.35 & 1.56 & 0.34 & -17.28 & 1.23 & 0.29 & -17.36 & 0.58 & -17.40 & 0.72 & $5.8 \pm 2.9^{\dagger}$ & $\ldots$ & $1.6 \pm 0.1$ \\
\hline SCOPEG172.88+02.27 & 0.65 & -18.27 & 1.78 & 0.52 & -17.43 & 2.24 & 0.21 & -17.45 & 2.14 & 0.25 & -16.89 & 1.58 & -17.30 & 1.79 & $5.8 \pm 2.9^{\dagger}$ & $\ldots$ & $1.0 \pm 0.1$ \\
\hline SCOPEG172.89+02.27 & 0.20 & -18.20 & 0.64 & $\leq 0.08$ & $\ldots$ & $\ldots$ & $\leq 0.09$ & $\ldots$ & $\ldots$ & $\leq 0.09$ & $\ldots$ & $\ldots$ & $\ldots$ & $\ldots$ & $\ldots$ & $\ldots$ & $\ldots$ \\
\hline SCOPEG172.89+02.27 & 0.73 & -16.73 & 0.97 & 0.88 & -16.65 & 1.46 & 0.36 & -16.65 & 0.92 & 0.25 & -16.74 & 2.00 & -16.70 & 1.07 & $5.8 \pm 2.9^{\dagger}$ & $\ldots$ & $1.6 \pm 0.1$ \\
\hline SCOPEG173.17+02.36 & 0.25 & -20.63 & 1.12 & 0.16 & -20.55 & 1.46 & $\leq 0.12$ & $\cdots$ & $\cdots$ & $\leq 0.12$ & $\cdots$ & $\cdots$ & $\cdots$ & $\cdots$ & $\cdots$ & $\cdots$ & $\cdots$ \\
\hline SCOPEG173.17+02.36 & 0.49 & -18.85 & 1.02 & 0.28 & -18.73 & 1.48 & 0.21 & -18.70 & 1.71 & $\leq 0.12$ & $\ldots$ & $\ldots$ & $\ldots$ & $\ldots$ & $\ldots$ & $\ldots$ & $\ldots$ \\
\hline SCOPEG173.18+02.35 & 0.10 & -21.25 & 0.63 & 0.13 & -20.98 & 1.45 & $\leq 0.12$ & $\ldots$ & $\ldots$ & $\leq 0.11$ & $\ldots$ & $\ldots$ & $\ldots$ & $\ldots$ & $\ldots$ & $\ldots$ & $\ldots$ \\
\hline SCOPEG173.18+02.35 & 0.54 & -18.96 & 1.31 & 0.40 & -18.96 & 1.73 & 0.33 & -18.83 & 1.57 & 0.14 & -19.13 & 0.94 & $\ldots$ & $\ldots$ & $7.4 \pm 3.7^{\dagger}$ & $\cdots$ & $\ldots$ \\
\hline SCOPEG173.19+02.35 & 0.65 & -19.55 & 1.51 & 0.43 & -19.42 & 1.92 & 0.32 & -19.32 & 1.52 & 0.15 & -19.27 & 0.94 & $\ldots$ & $\ldots$ & $7.4 \pm 3.7^{\dagger}$ & $\ldots$ & $\ldots$ \\
\hline SCOPEG178.27-00.60 & 0.34 & -0.88 & 0.76 & 0.49 & -0.67 & 1.11 & 0.15 & -0.79 & 0.66 & 0.24 & -0.84 & 0.53 & -0.78 & 0.58 & $4.0 \pm 0.2$ & $1.6 \pm 2.1$ & $2.0 \pm 0.4$ \\
\hline SCOPEG178.28-00.60 & 0.24 & -1.04 & 0.74 & 0.27 & -0.93 & 1.13 & 0.17 & -1.00 & 0.35 & 0.16 & -1.06 & 0.58 & $\ldots$ & $\ldots$ & $6.0 \pm 3.0^{\dagger}$ & $\ldots$ & $\ldots$ \\
\hline SCOPEG195.71-02.32 & 0.36 & 3.42 & 1.38 & 0.45 & 4.01 & 1.67 & 0.11 & 3.98 & 1.45 & 0.13 & 4.19 & 0.58 & $\ldots$ & $\ldots$ & $6.1 \pm 3.0^{\dagger}$ & $\cdots$ & $\ldots$ \\
\hline SCOPEG195.74-02.30 & 0.41 & 4.02 & 1.58 & 0.71 & 4.46 & 1.72 & 0.16 & 4.37 & 1.41 & 0.16 & 4.89 & 1.03 & $\ldots$ & $\ldots$ & $6.1 \pm 3.0^{\dagger}$ & $\ldots$ & $\ldots$ \\
\hline SCOPEG202.30+02.53 & 0.19 & 7.88 & 1.69 & 0.30 & 8.36 & 1.24 & 0.13 & 8.19 & 0.95 & 0.15 & 7.98 & 0.43 & 8.25 & 0.84 & $7.3 \pm 3.6^{\dagger}$ & $\ldots$ & $0.7 \pm 0.1$ \\
\hline
\end{tabular}

Table 4 continued 
Table 4 (continued)

\begin{tabular}{|c|c|c|c|c|c|c|c|c|c|c|c|c|c|c|c|c|c|}
\hline \multirow{3}{*}{ SCUBA-2 core } & \multicolumn{3}{|c|}{ c- $\mathrm{C}_{3} \mathrm{H}_{2}$} & \multicolumn{3}{|c|}{$\mathrm{DNC}$} & \multicolumn{3}{|c|}{$\mathrm{HN}^{13} \mathrm{C}$} & \multicolumn{8}{|c|}{$\mathrm{N}_{2} \mathrm{D}^{+}$} \\
\hline & \multirow{2}{*}{$\begin{array}{c}T_{\text {peak }} \\
(\mathrm{K})\end{array}$} & $V_{\mathrm{LSR}}$ & $\Delta v$ & \multirow{2}{*}{$\begin{array}{c}T_{\text {peak }} \\
(\mathrm{K})\end{array}$} & $V_{\mathrm{LSR}}$ & $\Delta v$ & \multirow{2}{*}{$\begin{array}{c}T_{\text {peak }} \\
(\mathrm{K})\end{array}$} & $V_{\mathrm{LSR}}$ & $\Delta v$ & \multirow{2}{*}{$\begin{array}{c}T_{\text {peak }} \\
(\mathrm{K})\end{array}$} & $V_{\mathrm{LSR}, \mathrm{GA}}$ & $\Delta v_{\mathrm{GA}}$ & $V_{\mathrm{LSR}, \mathrm{HFS}}$ & $\Delta v_{\mathrm{HFS}}$ & \multirow{3}{*}{$\begin{array}{l}T_{\text {ex }} \\
(\mathrm{K}) \\
(16)\end{array}$} & \multirow{3}{*}{$\begin{array}{c}\tau \\
(17)\end{array}$} & \multirow{3}{*}{$\begin{array}{r}T_{\text {ant }} \tau \\
(\mathrm{K}) \\
(18)\end{array}$} \\
\hline & & \multicolumn{2}{|c|}{$\left(\mathrm{km} \mathrm{s}^{-1}\right)$} & & \multicolumn{2}{|c|}{$\left(\mathrm{km} \mathrm{s}^{-1}\right)$} & & \multicolumn{2}{|c|}{$\left(\mathrm{km} \mathrm{s}^{-1}\right)$} & & & \multicolumn{2}{|c|}{$\left(\mathrm{km} \mathrm{s}^{-1}\right)$} & & & & \\
\hline (1) & (2) & (3) & (4) & (5) & (6) & (7) & (8) & (9) & $(10)$ & (11) & (12) & (13) & (14) & $(15)$ & & & \\
\hline SCOPEG202.31+02.52 & 0.17 & 7.07 & 2.18 & 0.25 & 7.49 & 2.23 & $\leq 0.10$ & $\cdots$ & $\cdots$ & 0.11 & 7.97 & 0.42 & $\cdots$ & $\cdots$ & $7.3 \pm 3.6^{\dagger}$ & $\cdots$ & $\cdots$ \\
\hline SCOPEG202.32+02.53 & 0.11 & 5.39 & 0.55 & $\leq 0.11$ & $\cdots$ & $\cdots$ & $\leq 0.10$ & $\cdots$ & $\cdots$ & $\leq 0.09$ & $\ldots$ & $\ldots$ & $\cdots$ & $\ldots$ & $\cdots$ & $\cdots$ & $\ldots$ \\
\hline SCOPEG202.32+02.53 & 0.35 & 7.06 & 0.82 & 0.70 & 7.11 & 1.19 & 0.14 & 7.14 & 0.91 & 0.20 & 7.05 & 0.63 & $\cdots$ & $\cdots$ & $7.3 \pm 3.6^{\dagger}$ & $\cdots$ & $\cdots$ \\
\hline SCOPEG006.01+36.74 & 0.81 & 2.32 & 0.25 & 1.30 & 2.43 & 0.82 & 0.45 & 2.38 & 0.51 & 0.61 & 2.43 & 0.31 & 2.43 & 0.27 & $6.3 \pm 0.4$ & $1.6 \pm 1.2$ & $5.6 \pm 0.6$ \\
\hline SCOPEG001.37+20.95 & 1.44 & 0.68 & 0.44 & 1.99 & 0.82 & 0.92 & 0.82 & 0.78 & 0.51 & 0.68 & 0.77 & 0.39 & 0.78 & 0.36 & $5.2 \pm 0.3$ & $2.8 \pm 1.3$ & $7.0 \pm 0.8$ \\
\hline SCOPEG010.19+02.41 & 0.19 & 5.68 & 1.20 & $\leq 0.16$ & $\cdots$ & $\cdots$ & $\leq 0.16$ & $\cdots$ & $\cdots$ & $\leq 0.17$ & $\cdots$ & $\cdots$ & $\cdots$ & $\cdots$ & $\cdots$ & $\cdots$ & $\cdots$ \\
\hline SCOPEG005.91-00.95 & $\leq 0.17$ & $\cdots$ & $\ldots$ & $\leq 0.26$ & .. & $\cdots$ & $\leq 0.19$ & $\cdots$ & $\ldots$ & $\leq 0.23$ & $\cdots$ & $\cdots$ & $\cdots$ & $\cdots$ & $\cdots$ & $\cdots$ & $\cdots$ \\
\hline SCOPEG005.88-01.01 & 0.24 & 12.40 & 1.53 & 0.21 & 12.44 & 0.83 & $\leq 0.14$ & $\cdots$ & $\cdots$ & $\leq 0.16$ & $\cdots$ & $\cdots$ & $\cdots$ & $\cdots$ & $\cdots$ & $\cdots$ & $\cdots$ \\
\hline SCOPEG005.92-00.99 & 0.21 & 12.43 & 1.57 & $\leq 0.22$ & $\cdots$ & $\cdots$ & $\leq 0.15$ & $\cdots$ & $\cdots$ & $\leq 0.17$ & $\cdots$ & $\cdots$ & $\cdots$ & $\cdots$ & $\cdots$ & $\cdots$ & $\cdots$ \\
\hline SCOPEG005.92-00.99 & 0.20 & 15.29 & 2.00 & $\leq 0.21$ & $\cdots$ & $\cdots$ & $\leq 0.15$ & $\cdots$ & $\cdots$ & $\leq 0.17$ & $\cdots$ & $\cdots$ & $\cdots$ & $\cdots$ & $\cdots$ & $\cdots$ & $\cdots$ \\
\hline SCOPEG005.90-01.01 & 0.18 & 12.36 & 2.19 & $\leq 0.20$ & .. & $\cdots$ & $\leq 0.14$ & $\cdots$ & $\cdots$ & $\leq 0.16$ & $\cdots$ & $\cdots$ & $\cdots$ & $\cdots$ & $\cdots$ & $\cdots$ & $\cdots$ \\
\hline SCOPEG005.90-01.01 & 0.20 & 15.72 & 1.37 & $\leq 0.19$ & $\cdots$ & $\cdots$ & $\leq 0.14$ & $\cdots$ & $\cdots$ & $\leq 0.16$ & $\cdots$ & $\cdots$ & $\cdots$ & $\cdots$ & $\cdots$ & $\cdots$ & $\cdots$ \\
\hline SCOPEG005.91-01.02 & 0.18 & 11.24 & 0.40 & $\leq 0.17$ & $\cdots$ & $\cdots$ & $\leq 0.15$ & $\cdots$ & $\cdots$ & $\leq 0.18$ & $\cdots$ & $\cdots$ & $\cdots$ & $\cdots$ & $\cdots$ & $\cdots$ & $\cdots$ \\
\hline SCOPEG005.91-01.02 & $\leq 0.14$ & $\cdots$ & $\cdots$ & $\leq 0.17$ & $\cdots$ & $\cdots$ & $\leq 0.15$ & $\cdots$ & $\cdots$ & $\leq 0.18$ & $\cdots$ & $\cdots$ & $\cdots$ & $\cdots$ & $\cdots$ & $\cdots$ & $\cdots$ \\
\hline SCOPEG017.38+02.26 & 0.24 & 10.74 & 0.70 & 0.17 & 10.87 & 1.04 & 0.15 & 10.87 & 0.64 & $\leq 0.17$ & $\cdots$ & $\cdots$ & $\cdots$ & $\cdots$ & $\cdots$ & $\cdots$ & $\cdots$ \\
\hline SCOPEG017.38+02.25 & 0.20 & 10.84 & 1.39 & 0.17 & 10.74 & 1.33 & $\leq 0.13$ & $\cdots$ & $\cdots$ & $\leq 0.13$ & $\cdots$ & $\cdots$ & $\cdots$ & $\cdots$ & $\cdots$ & $\cdots$ & $\cdots$ \\
\hline SCOPEG017.37+02.24 & 0.26 & 10.52 & 1.33 & 0.16 & 10.83 & 1.32 & 0.18 & 10.54 & 1.01 & $\leq 0.13$ & $\cdots$ & $\cdots$ & $\cdots$ & $\cdots$ & $\cdots$ & $\cdots$ & $\cdots$ \\
\hline SCOPEG017.36+02.23 & 0.32 & 10.31 & 1.32 & 0.20 & 10.41 & 1.06 & 0.23 & 10.36 & 1.55 & 0.14 & 9.98 & 0.33 & 9.93 & 0.35 & $7.7 \pm 3.9^{\dagger}$ & $\cdots$ & $0.9 \pm 0.2$ \\
\hline SCOPEG014.20-00.18 & 0.19 & 37.19 & 0.77 & $\leq 0.15$ & $\cdots$ & $\cdots$ & $\leq 0.15$ & $\cdots$ & $\cdots$ & $\leq 0.16$ & $\cdots$ & $\cdots$ & $\cdots$ & $\cdots$ & $\cdots$ & $\cdots$ & $\cdots$ \\
\hline SCOPEG014.20-00.18 & $\leq 0.13$ & $\cdots$ & $\cdots$ & $\leq 0.15$ & $\cdots$ & $\cdots$ & 0.16 & 39.27 & 0.68 & $\leq 0.16$ & $\cdots$ & $\cdots$ & $\cdots$ & $\cdots$ & $\cdots$ & $\cdots$ & $\cdots$ \\
\hline SCOPEG014.23-00.17 & 0.15 & 37.50 & 2.78 & $\leq 0.15$ & $\cdots$ & $\cdots$ & 0.20 & 37.54 & 1.97 & $\leq 0.14$ & $\cdots$ & $\cdots$ & $\cdots$ & $\cdots$ & $\cdots$ & $\cdots$ & $\cdots$ \\
\hline SCOPEG014.18-00.23 & 0.16 & 39.29 & 0.96 & $\leq 0.12$ & $\cdots$ & $\cdots$ & 0.18 & 39.23 & 0.43 & $\leq 0.12$ & $\cdots$ & $\cdots$ & $\ldots$ & $\cdots$ & $\cdots$ & $\cdots$ & $\cdots$ \\
\hline SCOPEG014.18-00.23 & 0.24 & 40.46 & 0.79 & 0.12 & 40.60 & 1.77 & 0.18 & 40.60 & 1.03 & $\leq 0.12$ & $\cdots$ & $\cdots$ & $\ldots$ & $\cdots$ & $\cdots$ & $\cdots$ & $\cdots$ \\
\hline SCOPEG014.71-00.15 & 0.48 & 40.89 & 1.98 & $\leq 0.23$ & $\cdots$ & $\cdots$ & $\leq 0.21$ & $\cdots$ & $\cdots$ & $\leq 0.26$ & $\cdots$ & $\cdots$ & $\cdots$ & $\cdots$ & $\cdots$ & $\cdots$ & $\cdots$ \\
\hline SCOPEG014.72-00.20 & 0.26 & 37.53 & 1.30 & 0.21 & 37.44 & 0.30 & 0.23 & 37.62 & 1.54 & $\leq 0.20$ & $\cdots$ & $\cdots$ & $\cdots$ & $\cdots$ & $\cdots$ & $\cdots$ & $\cdots$ \\
\hline SCOPEG014.69-00.22 & $\leq 0.13$ & $\cdots$ & $\cdots$ & $\leq 0.20$ & $\cdots$ & $\cdots$ & $\leq 0.16$ & $\cdots$ & $\cdots$ & $\leq 0.18$ & $\cdots$ & $\cdots$ & $\cdots$ & $\cdots$ & $\cdots$ & $\cdots$ & $\cdots$ \\
\hline SCOPEG014.71-00.22 & 0.35 & 36.97 & 1.60 & $\leq 0.20$ & $\cdots$ & $\cdots$ & 0.24 & 37.31 & 2.14 & $\leq 0.18$ & $\cdots$ & $\cdots$ & $\cdots$ & $\cdots$ & $\cdots$ & $\cdots$ & $\cdots$ \\
\hline SCOPEG014.11-00.57 & 0.30 & 20.50 & 3.76 & 0.28 & 20.67 & 3.65 & 0.24 & 20.11 & 3.32 & $\leq 0.17$ & $\cdots$ & $\cdots$ & $\cdots$ & $\cdots$ & $\cdots$ & $\cdots$ & $\cdots$ \\
\hline SCOPEG014.23-00.51 & 0.33 & 19.65 & 2.69 & 0.34 & 19.62 & 2.60 & 0.21 & 19.33 & 2.61 & $\leq 0.17$ & $\cdots$ & $\cdots$ & $\cdots$ & $\cdots$ & $\cdots$ & $\cdots$ & $\cdots$ \\
\hline SCOPEG016.93+00.28 & 0.19 & 24.24 & 2.20 & $\leq 0.21$ & $\cdots$ & $\cdots$ & $\leq 0.19$ & $\cdots$ & $\cdots$ & $\leq 0.23$ & $\cdots$ & $\cdots$ & $\cdots$ & $\cdots$ & $\cdots$ & $\cdots$ & $\cdots$ \\
\hline SCOPEG016.93+00.27 & 0.25 & 24.08 & 2.30 & $\leq 0.18$ & $\cdots$ & $\cdots$ & $\leq 0.15$ & $\cdots$ & $\cdots$ & $\leq 0.16$ & $\cdots$ & $\cdots$ & $\cdots$ & $\cdots$ & $\cdots$ & $\cdots$ & $\cdots$ \\
\hline SCOPEG016.93+00.25 & 0.41 & 24.18 & 0.98 & 0.29 & 24.52 & 1.50 & 0.21 & 24.37 & 0.86 & $\leq 0.16$ & $\cdots$ & $\cdots$ & $\cdots$ & $\cdots$ & $\cdots$ & $\cdots$ & $\cdots$ \\
\hline SCOPEG016.93+00.25 & 0.18 & 26.04 & 0.88 & $\leq 0.17$ & $\cdots$ & $\cdots$ & $\leq 0.14$ & $\cdots$ & $\cdots$ & $\leq 0.16$ & $\cdots$ & $\cdots$ & $\cdots$ & $\cdots$ & $\cdots$ & $\cdots$ & $\cdots$ \\
\hline SCOPEG016.93+00.24 & 0.33 & 24.28 & 1.23 & 0.20 & 24.88 & 0.39 & 0.20 & 24.53 & 0.63 & $\leq 0.16$ & $\cdots$ & $\cdots$ & $\cdots$ & $\cdots$ & $\cdots$ & $\cdots$ & $\cdots$ \\
\hline SCOPEG016.93+00.24 & 0.28 & 26.28 & 0.77 & 0.17 & 26.19 & 0.49 & 0.19 & 26.40 & 0.56 & $\leq 0.16$ & $\cdots$ & $\cdots$ & $\ldots$ & $\cdots$ & $\cdots$ & $\cdots$ & $\cdots$ \\
\hline SCOPEG016.92+00.23 & 0.20 & 24.19 & 2.98 & $\leq 0.15$ & $\ldots$ & $\ldots$ & $\leq 0.15$ & $\ldots$ & $\ldots$ & $\leq 0.16$ & $\ldots$ & $\ldots$ & $\ldots$ & $\ldots$ & $\ldots$ & $\ldots$ & $\ldots$ \\
\hline
\end{tabular}

Table 4 continued 
Table 4 (continued)

\begin{tabular}{|c|c|c|c|c|c|c|c|c|c|c|c|c|c|c|c|c|c|}
\hline \multirow{3}{*}{ SCUBA-2 core } & \multicolumn{3}{|c|}{ c- $\mathrm{C}_{3} \mathrm{H}_{2}$} & \multicolumn{3}{|c|}{$\mathrm{DNC}$} & \multicolumn{3}{|c|}{$\mathrm{HN}^{13} \mathrm{C}$} & \multicolumn{8}{|c|}{$\mathrm{N}_{2} \mathrm{D}^{+}$} \\
\hline & \multirow{2}{*}{$\begin{array}{c}T_{\text {peak }} \\
(\mathrm{K})\end{array}$} & $V_{\mathrm{LSR}}$ & $\Delta v$ & \multirow{2}{*}{$\begin{array}{c}T_{\text {peak }} \\
(\mathrm{K})\end{array}$} & $V_{\mathrm{LSR}}$ & $\Delta v$ & \multirow{2}{*}{$\begin{array}{c}T_{\text {peak }} \\
(\mathrm{K})\end{array}$} & $V_{\mathrm{LSR}}$ & $\Delta v$ & \multirow{2}{*}{$\begin{array}{c}T_{\text {peak }} \\
(\mathrm{K})\end{array}$} & \multirow[t]{2}{*}{$V_{\mathrm{LSR}, \mathrm{GA}}$} & $\Delta v_{\mathrm{GA}}$ & $V_{\mathrm{LSR}, \mathrm{HFS}}$ & $\Delta v_{\mathrm{HFS}}$ & \multirow{3}{*}{$\begin{array}{l}T_{\text {ex }} \\
(\mathrm{K}) \\
(16)\end{array}$} & \multirow{3}{*}{$\begin{array}{c}\tau \\
(17) \\
\end{array}$} & \multirow{3}{*}{$\begin{array}{c}T_{\text {ant }} \tau \\
(\mathrm{K}) \\
(18)\end{array}$} \\
\hline & & \multicolumn{2}{|c|}{$\left(\mathrm{km} \mathrm{s}^{-1}\right)$} & & \multicolumn{2}{|c|}{$\left(\mathrm{km} \mathrm{s}^{-1}\right)$} & & \multicolumn{2}{|c|}{$\left(\mathrm{km} \mathrm{s}^{-1}\right)$} & & & \multicolumn{2}{|c|}{$\left(\mathrm{km} \mathrm{s}^{-1}\right)$} & & & & \\
\hline$(1)$ & (2) & (3) & $(4)$ & $(5)$ & (6) & $(7)$ & $(8)$ & $(9)$ & $(10)$ & $(11)$ & $(12)$ & $(13)$ & $(14)$ & $(15)$ & & & \\
\hline SCOPEG016.93+00.22 & 0.33 & 23.74 & 1.34 & 0.44 & 23.74 & 1.15 & 0.25 & 23.77 & 1.39 & $\leq 0.15$ & $\cdots$ & $\cdots$ & $\cdots$ & $\cdots$ & $\cdots$ & $\cdots$ & $\cdots$ \\
\hline SCOPEG016.30-00.53 & $\leq 0.18$ & $\ldots$ & $\ldots$ & $\leq 0.26$ & $\ldots$ & ... & 0.20 & 38.04 & 0.72 & $\leq 0.23$ & $\ldots$ & $\ldots$ & .. & $\ldots$ & $\ldots$ & $\ldots$ & $\ldots$ \\
\hline SCOPEG016.34-00.59 & 0.35 & 41.25 & 1.85 & $\leq 0.18$ & $\cdots$ & $\cdots$ & 0.25 & 41.34 & 2.36 & $\leq 0.18$ & $\cdots$ & $\cdots$ & $\cdots$ & $\cdots$ & $\cdots$ & $\cdots$ & $\cdots$ \\
\hline SCOPEG016.38-00.61 & 0.27 & 41.37 & 1.41 & $\leq 0.17$ & $\ldots$ & $\cdots$ & 0.16 & 41.44 & 2.35 & $\leq 0.17$ & $\cdots$ & $\ldots$ & $\ldots$ & $\cdots$ & $\ldots$ & $\cdots$ & $\ldots$ \\
\hline SCOPEG016.42-00.64 & 0.33 & 40.42 & 1.23 & $\leq 0.16$ & $\cdots$ & $\cdots$ & 0.25 & 40.64 & 1.22 & $\leq 0.17$ & $\cdots$ & $\cdots$ & $\cdots$ & $\cdots$ & $\ldots$ & $\cdots$ & $\cdots$ \\
\hline SCOPEG016.42-00.64 & 0.19 & 42.04 & 1.30 & $\leq 0.16$ & $\cdots$ & $\cdots$ & 0.22 & 42.22 & 0.37 & $\leq 0.17$ & $\cdots$ & $\cdots$ & $\cdots$ & $\cdots$ & $\cdots$ & $\cdots$ & $\cdots$ \\
\hline SCOPEG017.22-01.46 & $\leq 0.15$ & $\ldots$ & $\ldots$ & $\leq 0.19$ & $\cdots$ & $\cdots$ & $\leq 0.15$ & $\cdots$ & $\ldots$ & $\leq 0.17$ & $\cdots$ & $\cdots$ & $\ldots$ & $\cdots$ & $\ldots$ & $\cdots$ & $\ldots$ \\
\hline SCOPEG017.21-01.47 & 0.15 & 34.21 & 1.98 & $\leq 0.19$ & $\ldots$ & $\cdots$ & $\leq 0.16$ & $\ldots$ & $\ldots$ & $\leq 0.16$ & $\cdots$ & $\cdots$ & $\cdots$ & $\cdots$ & $\ldots$ & $\cdots$ & $\ldots$ \\
\hline SCOPEG023.63+00.59 & 0.17 & 96.72 & 0.68 & $\leq 0.17$ & $\cdots$ & $\cdots$ & $\leq 0.14$ & $\cdots$ & $\cdots$ & $\leq 0.16$ & $\cdots$ & $\cdots$ & $\cdots$ & $\cdots$ & $\cdots$ & $\cdots$ & $\cdots$ \\
\hline SCOPEG023.69+00.59 & 0.13 & 97.69 & 2.83 & $\leq 0.16$ & $\ldots$ & $\cdots$ & 0.17 & 97.92 & 2.61 & $\leq 0.16$ & $\cdots$ & $\cdots$ & $\cdots$ & $\cdots$ & $\ldots$ & $\cdots$ & $\ldots$ \\
\hline SCOPEG024.02+00.24 & $\leq 0.15$ & $\cdots$ & $\cdots$ & $\leq 0.17$ & $\cdots$ & $\cdots$ & $\leq 0.15$ & $\cdots$ & $\cdots$ & $\leq 0.15$ & $\cdots$ & $\cdots$ & $\cdots$ & $\cdots$ & $\cdots$ & $\cdots$ & $\cdots$ \\
\hline SCOPEG024.02+00.21 & 0.12 & 105.80 & 2.55 & $\leq 0.14$ & $\ldots$ & $\cdots$ & $\leq 0.13$ & $\ldots$ & $\ldots$ & $\leq 0.14$ & $\ldots$ & $\ldots$ & $\cdots$ & $\cdots$ & $\ldots$ & $\cdots$ & $\ldots$ \\
\hline SCOPEG023.32-00.29 & 0.13 & 102.10 & 2.53 & $\leq 0.16$ & $\cdots$ & $\cdots$ & 0.24 & 102.47 & 1.69 & $\leq 0.17$ & $\cdots$ & $\cdots$ & $\cdots$ & $\cdots$ & $\cdots$ & $\cdots$ & $\cdots$ \\
\hline SCOPEG026.50+00.71 & 0.23 & 48.18 & 2.86 & $\leq 0.20$ & $\ldots$ & $\cdots$ & 0.15 & 48.75 & 2.48 & $\leq 0.17$ & $\cdots$ & $\ldots$ & $\cdots$ & . & $\ldots$ & $\cdots$ & $\ldots$ \\
\hline SCOPEG037.92+02.14 & 0.14 & 34.29 & 0.85 & $\leq 0.11$ & $\cdots$ & $\cdots$ & 0.08 & 34.32 & 0.75 & $\leq 0.10$ & $\cdots$ & $\cdots$ & $\cdots$ & $\cdots$ & $\cdots$ & $\cdots$ & $\cdots$ \\
\hline SCOPEG033.74-00.01 & 0.24 & 106.00 & 2.93 & 0.12 & 105.89 & 2.50 & 0.26 & 106.07 & 3.06 & $\leq 0.11$ & $\ldots$ & $\ldots$ & $\cdots$ & $\cdots$ & $\ldots$ & $\cdots$ & $\ldots$ \\
\hline SCOPEG039.74+01.99 & 0.43 & 30.61 & 1.06 & 0.24 & 30.66 & 1.40 & 0.16 & 30.64 & 0.98 & $\leq 0.10$ & $\cdots$ & $\cdots$ & $\cdots$ & $\cdots$ & $\cdots$ & $\cdots$ & $\cdots$ \\
\hline SCOPEG035.48-00.29 & 0.24 & 42.95 & 1.02 & 0.18 & 42.83 & 1.26 & $\leq 0.13$ & $\ldots$ & $\ldots$ & $\leq 0.14$ & $\cdots$ & $\cdots$ & $\cdots$ & $\cdots$ & $\ldots$ & $\cdots$ & $\ldots$ \\
\hline SCOPEG035.48-00.29 & 0.52 & 45.29 & 1.26 & 0.53 & 45.63 & 1.42 & 0.36 & 45.48 & 1.15 & 0.18 & 45.77 & 0.76 & 45.70 & 0.68 & $7.0 \pm 3.5^{\dagger}$ & $\cdots$ & $1.2 \pm 0.1$ \\
\hline SCOPEG035.52-00.27 & 0.55 & 45.51 & 1.79 & 0.46 & 45.97 & 2.14 & 0.31 & 45.82 & 1.96 & $\leq 0.12$ & $\cdots$ & $\ldots$ & $\cdots$ & $\cdots$ & $\ldots$ & $\cdots$ & $\ldots$ \\
\hline SCOPEG035.48-00.31 & 0.47 & 44.85 & 1.63 & 0.50 & 45.03 & 1.64 & 0.31 & 44.99 & 1.72 & 0.19 & 44.88 & 0.95 & $\ldots$ & $\ldots$ & $7.0 \pm 3.5^{\dagger}$ & $\ldots$ & $\ldots$ \\
\hline SCOPEG034.75-01.38 & 0.41 & 45.68 & 2.18 & 0.30 & 45.95 & 2.13 & 0.33 & 45.74 & 2.14 & $\leq 0.13$ & $\ldots$ & $\ldots$ & $\ldots$ & $\ldots$ & $\ldots$ & $\cdots$ & $\ldots$ \\
\hline SCOPEG035.36-01.77 & 0.14 & 42.25 & 3.25 & $\leq 0.13$ & $\cdots$ & $\cdots$ & 0.11 & 42.40 & 0.64 & $\leq 0.12$ & $\cdots$ & $\cdots$ & $\cdots$ & $\cdots$ & $\cdots$ & $\cdots$ & $\cdots$ \\
\hline SCOPEG035.36-01.78 & 0.20 & 42.41 & 2.28 & 0.24 & 42.68 & 1.19 & $\leq 0.09$ & $\ldots$ & $\ldots$ & $\leq 0.10$ & $\ldots$ & $\ldots$ & $\ldots$ & $\ldots$ & $\ldots$ & $\cdots$ & $\ldots$ \\
\hline SCOPEG035.35-01.80 & 0.16 & 41.87 & 2.32 & 0.11 & 41.97 & 1.90 & $\leq 0.08$ & $\ldots$ & $\ldots$ & $\leq 0.10$ & $\cdots$ & $\ldots$ & $\ldots$ & $\ldots$ & $\ldots$ & .. & $\ldots$ \\
\hline SCOPEG057.11+03.66 & 0.47 & 12.08 & 0.55 & 0.51 & 12.28 & 1.04 & 0.28 & 12.21 & 0.78 & 0.18 & 12.12 & 0.48 & 12.20 & 0.47 & $5.9 \pm 3.0^{\dagger}$ & $\cdots$ & $1.1 \pm 0.1$ \\
\hline SCOPEG057.10+03.63 & 0.94 & 11.97 & 0.59 & 0.59 & 12.11 & 1.04 & 0.35 & 12.03 & 0.81 & 0.14 & 11.90 & 0.33 & 12.10 & 0.61 & $5.9 \pm 3.0^{\dagger}$ & $\cdots$ & $0.6 \pm 0.1$ \\
\hline SCOPEG069.80-01.67 & 0.44 & 12.29 & 1.73 & 0.39 & 12.47 & 1.34 & 0.27 & 12.47 & 1.59 & $\leq 0.11$ & $\ldots$ & $\cdots$ & $\ldots$ & $\ldots$ & $\ldots$ & $\ldots$ & $\ldots$ \\
\hline SCOPEG069.81-01.67 & 0.70 & 11.81 & 1.39 & 0.36 & 11.95 & 1.44 & 0.30 & 11.95 & 1.36 & 0.10 & 11.71 & 0.40 & $\cdots$ & $\cdots$ & $7.1 \pm 3.5^{\dagger}$ & $\cdots$ & $\cdots$ \\
\hline SCOPEG070.40-01.39 & 0.31 & 11.98 & 1.11 & 0.16 & 12.08 & 1.18 & 0.18 & 12.05 & 0.99 & $\leq 0.09$ & $\ldots$ & $\cdots$ & $\ldots$ & $\ldots$ & $\cdots$ & $\cdots$ & $\ldots$ \\
\hline SCOPEG074.10+00.11 & 0.44 & -2.52 & 1.66 & 0.19 & -2.21 & 2.31 & 0.17 & -2.19 & 1.75 & 0.10 & -1.77 & 0.67 & -2.20 & 1.33 & $6.2 \pm 3.1^{\dagger}$ & $\cdots$ & $0.3 \pm 0.1$ \\
\hline SCOPEG074.11+00.11 & 0.39 & -1.14 & 2.60 & 0.34 & -0.65 & 2.25 & 0.15 & -0.95 & 2.45 & 0.14 & -0.54 & 0.61 & -0.88 & 1.80 & $6.2 \pm 3.1^{\dagger}$ & $\ldots$ & $0.5 \pm 0.1$ \\
\hline SCOPEG082.36-01.83 & 0.60 & 4.23 & 0.59 & 0.55 & 4.46 & 1.01 & 0.31 & 4.43 & 0.77 & 0.18 & 4.54 & 0.42 & $\ldots$ & $\ldots$ & $5.7 \pm 2.9^{\dagger}$ & $\ldots$ & $\ldots$ \\
\hline SCOPEG082.40-01.84 & 0.77 & 5.48 & 0.60 & 0.52 & 5.50 & 1.32 & 0.33 & 5.47 & 0.96 & 0.19 & 5.40 & 0.55 & $\ldots$ & $\ldots$ & $5.7 \pm 2.9^{\dagger}$ & $\ldots$ & $\ldots$ \\
\hline SCOPEG082.41-01.84 & 0.50 & 4.69 & 0.87 & 0.84 & 4.92 & 1.09 & 0.40 & 4.98 & 1.09 & 0.26 & 4.88 & 0.60 & 4.90 & 0.60 & $5.7 \pm 2.9^{\dagger}$ & $\cdots$ & $1.7 \pm 0.1$ \\
\hline SCOPEG082.42-01.84 & 0.39 & 5.50 & 0.93 & 0.65 & 5.17 & 1.25 & 0.33 & 5.26 & 1.16 & 0.24 & 4.91 & 0.59 & 5.10 & 0.92 & $6.1 \pm 0.1$ & $0.4 \pm 0.1$ & $1.4 \pm 0.1$ \\
\hline
\end{tabular}

Table 4 continued 
Table 4 (continued)

\begin{tabular}{|c|c|c|c|c|c|c|c|c|c|c|c|c|c|c|c|c|c|}
\hline \multirow{3}{*}{ SCUBA-2 core } & \multicolumn{3}{|c|}{ c- $\mathrm{C}_{3} \mathrm{H}_{2}$} & \multicolumn{3}{|c|}{$\mathrm{DNC}$} & \multicolumn{3}{|c|}{$\mathrm{HN}^{13} \mathrm{C}$} & \multicolumn{8}{|c|}{$\mathrm{N}_{2} \mathrm{D}^{+}$} \\
\hline & \multirow{2}{*}{$\begin{array}{c}T_{\text {peak }} \\
(\mathrm{K})\end{array}$} & $V_{\mathrm{LSR}}$ & $\Delta v$ & \multirow{2}{*}{$\begin{array}{c}T_{\text {peak }} \\
(\mathrm{K})\end{array}$} & $V_{\mathrm{LSR}}$ & $\Delta v$ & \multirow{2}{*}{$\begin{array}{c}T_{\text {peak }} \\
(\mathrm{K})\end{array}$} & $V_{\mathrm{LSR}}$ & $\Delta v$ & \multirow{2}{*}{$\begin{array}{c}T_{\text {peak }} \\
(\mathrm{K})\end{array}$} & $V_{\mathrm{LSR}, \mathrm{GA}}$ & $\Delta v_{\mathrm{GA}}$ & $V_{\mathrm{LSR}, \mathrm{HFS}}$ & $\Delta v_{\mathrm{HFS}}$ & \multirow{3}{*}{$\begin{array}{l}T_{\mathrm{ex}} \\
(\mathrm{K}) \\
(16)\end{array}$} & \multirow{3}{*}{$\begin{array}{c}\tau \\
(17)\end{array}$} & \multirow{3}{*}{$\begin{array}{c}T_{\text {ant }} \tau \\
(\mathrm{K}) \\
(18)\end{array}$} \\
\hline & & \multicolumn{2}{|c|}{$\left(\mathrm{km} \mathrm{s}^{-1}\right)$} & & \multicolumn{2}{|c|}{$\left(\mathrm{km} \mathrm{s}^{-1}\right)$} & & \multicolumn{2}{|c|}{$\left(\mathrm{km} \mathrm{s}^{-1}\right)$} & & & \multicolumn{2}{|c|}{$\left(\mathrm{km} \mathrm{s}^{-1}\right)$} & & & & \\
\hline (1) & (2) & (3) & (4) & (5) & (6) & $(7)$ & (8) & (9) & $(10)$ & (11) & (12) & (13) & (14) & (15) & & & \\
\hline SCOPEG091.86+04.17 & 0.68 & -3.78 & 0.61 & 0.32 & -3.64 & 1.08 & 0.23 & -3.68 & 0.80 & $\leq 0.12$ & $\cdots$ & $\cdots$ & $\cdots$ & $\cdots$ & $\cdots$ & $\cdots$ & $\cdots$ \\
\hline SCOPEG091.86+04.17 & 0.30 & -1.27 & 0.22 & $\leq 0.13$ & $\ldots$ & $\cdots$ & $\leq 0.13$ & $\cdots$ & .. & $\leq 0.12$ & $\cdots$ & $\cdots$ & $\cdots$ & $\cdots$ & $\cdots$ & $\cdots$ & $\cdots$ \\
\hline SCOPEG091.85+04.12 & 1.11 & -2.85 & 0.89 & 0.59 & -2.74 & 1.41 & 0.33 & -2.74 & 1.04 & 0.15 & -2.81 & 0.73 & -2.71 & 0.66 & $8.4 \pm 1.6$ & $0.2 \pm 10.6$ & $1.1 \pm 0.3$ \\
\hline SCOPEG092.03+03.93 & 0.11 & -1.82 & 0.37 & $\leq 0.10$ & $\ldots$ & $\ldots$ & $\leq 0.10$ & $\cdots$ & $\cdots$ & $\leq 0.08$ & $\cdots$ & $\cdots$ & $\cdots$ & $\cdots$ & $\cdots$ & $\cdots$ & .. \\
\hline SCOPEG092.27+03.79 & 0.59 & -2.48 & 1.40 & 0.46 & -2.16 & 1.42 & 0.34 & -2.31 & 1.35 & 0.18 & -2.08 & 0.67 & -2.08 & 0.70 & $4.3 \pm 0.1$ & $0.8 \pm 0.1$ & $1.2 \pm 0.1$ \\
\hline SCOPEG087.06-04.19 & 0.54 & 5.38 & 0.56 & 0.43 & 5.33 & 0.79 & 0.21 & 5.39 & 0.60 & 0.09 & 5.14 & 0.45 & 5.27 & 0.38 & $7.2 \pm 3.6^{\dagger}$ & $\ldots$ & $0.6 \pm 0.1$ \\
\hline SCOPEG089.64-06.62 & 0.90 & 12.27 & 0.63 & 0.46 & 12.33 & 1.00 & 0.21 & 12.30 & 0.80 & 0.12 & 12.61 & 0.34 & $\cdots$ & $\cdots$ & $5.6 \pm 2.8^{\dagger}$ & $\cdots$ & $\cdots$ \\
\hline SCOPEG105.37+09.84 & 0.15 & -9.94 & 1.32 & 0.23 & -9.59 & 1.48 & 0.14 & -9.56 & 1.34 & $\leq 0.11$ & $\cdots$ & $\cdots$ & $\cdots$ & $\ldots$ & $\cdots$ & $\cdots$ & $\cdots$ \\
\hline SCOPEG105.41+09.88 & $\leq 0.08$ & $\cdots$ & $\cdots$ & $\leq 0.10$ & $\ldots$ & $\cdots$ & $\leq 0.10$ & $\cdots$ & $\cdots$ & $\leq 0.10$ & $\cdots$ & $\cdots$ & $\cdots$ & $\cdots$ & .. & $\cdots$ & .. \\
\hline SCOPEG093.53-04.26 & 0.80 & 4.31 & 0.90 & 0.73 & 4.41 & 1.19 & 0.45 & 4.37 & 1.08 & 0.19 & 4.09 & 0.94 & 4.21 & 0.73 & $5.5 \pm 2.8^{\dagger}$ & $\ldots$ & $1.4 \pm 0.1$ \\
\hline SCOPEG093.54-04.28 & 0.69 & 4.04 & 0.82 & 0.72 & 4.20 & 1.20 & 0.34 & 4.12 & 1.02 & 0.20 & 4.00 & 1.01 & 4.09 & 0.68 & $5.5 \pm 2.8^{\dagger}$ & $\cdots$ & $1.3 \pm 0.1$ \\
\hline SCOPEG107.16+05.45 & 0.47 & -10.26 & 0.80 & 0.85 & -10.08 & 1.23 & 0.29 & -10.12 & 0.69 & 0.23 & -10.23 & 0.44 & $\cdots$ & $\cdots$ & $7.9 \pm 4.0^{\dagger}$ & $\cdots$ & $\cdots$ \\
\hline SCOPEG107.30+05.64 & 0.39 & -11.10 & 1.76 & 0.33 & -11.11 & 1.48 & 0.22 & -11.16 & 1.56 & $\leq 0.09$ & $\cdots$ & $\cdots$ & $\cdots$ & $\cdots$ & $\cdots$ & $\cdots$ & .. \\
\hline SCOPEG107.18+05.43 & 0.54 & -10.80 & 0.93 & 0.30 & -10.74 & 1.34 & 0.25 & -10.72 & 0.88 & 0.10 & -11.10 & 0.58 & $\ldots$ & $\ldots$ & $7.9 \pm 4.0^{\dagger}$ & $\ldots$ & . \\
\hline SCOPEG109.81+02.70 & 0.40 & -9.13 & 1.18 & 0.38 & -9.02 & 1.21 & 0.19 & -9.02 & 0.86 & $\leq 0.10$ & $\ldots$ & $\ldots$ & $\ldots$ & $\ldots$ & $\ldots$ & $\ldots$ & .. \\
\hline
\end{tabular}

Note-All values are measured in a spectrum whose peak temperature is higher than $3 \sigma$. In the case of a peak temperature below $3 \sigma$, the $3 \sigma$ level is listed as an upper limit. Column 1: SCUBA-2 core name, Column 2-4: Peak temperature at the $T_{\mathrm{A}}^{*}$ scale, systemic velocity, Full Width of DNC, Column 8-10: Same as Column 2-4 but of $\mathrm{HN}^{13} \mathrm{C}$, Column 11-13: Peak temperature at the $T_{\mathrm{A}}^{*}$ scale, systemic velocity, and FWHM inferred by Causian fitting to the brightest hyperfing inferr to line $\left(T_{\text {ex }}\right)$, total line option the structure fitting to seven components of the $\mathrm{N}_{2} \mathrm{D}^{+}$line. In columns 16 and 17, temperature. 
Table 5. Column density, column-density ratio, Mach number, and integrated intensity ratio of $\mathrm{N}_{2} \mathrm{D}^{+}, \mathrm{N}_{2} \mathrm{H}^{+}, \mathrm{DNC} \mathrm{HN}^{13} \mathrm{C}, \mathrm{CCS}$, and $\mathrm{HC} \mathrm{N}_{\text {molecules }}$

\begin{tabular}{|c|c|c|c|c|c|c|c|c|c|c|c|c|}
\hline SCUBA-2 core & $\begin{array}{c}N\left(\mathrm{~N}_{2} \mathrm{D}^{+}\right) \\
\left(10^{11} \mathrm{~cm}^{-2}\right) \\
(2)\end{array}$ & $\begin{array}{c}N\left(\mathrm{~N}_{2} \mathrm{H}^{+}\right) \\
\left(10^{12} \mathrm{~cm}^{-2}\right) \\
(3)\end{array}$ & $\begin{array}{c}N(\mathrm{DNC}) \\
\left(10^{11} \mathrm{~cm}^{-2}\right) \\
(4)\end{array}$ & $\begin{array}{c}N\left(\mathrm{HN}^{13} \mathrm{C}\right) \\
\left(10^{11} \mathrm{~cm}^{-2}\right) \\
(5)\end{array}$ & $\begin{array}{c}N(\mathrm{CCS}) \\
\left(10^{11} \mathrm{~cm}^{-2}\right) \\
(6)\end{array}$ & $\begin{array}{c}N\left(\mathrm{HC}_{3} \mathrm{~N}\right) \\
\left(10^{11} \mathrm{~cm}^{-2}\right) \\
(7)\end{array}$ & $\frac{N\left(\mathrm{~N}_{2} \mathrm{D}^{+}\right)}{N\left(\mathrm{~N}_{2} \mathrm{H}^{+}\right)}$ & $\frac{N(\mathrm{DNC})}{N\left(\mathrm{HN}^{13} \mathrm{C}\right)}$ & $\frac{N\left(\mathrm{~N}_{2} \mathrm{H}^{+}\right)}{N(\mathrm{CCS})}$ & $\frac{N\left(\mathrm{~N}_{2} \mathrm{H}^{+}\right)}{N\left(\mathrm{HC}_{3} \mathrm{~N}\right)}$ & (12) & $\frac{\int T(\mathrm{DNC}) d v}{\int T\left(\mathrm{HN}^{13} \mathrm{C}\right) d v}$ \\
\hline G190.15-13.75North & $\leq 15.0$ & $\leq 1.8$ & $\leq 4.8$ & $\leq 3.8$ & $\leq 53.0$ & $\leq 62.0$ & $\cdots$ & $\cdots$ & $\cdots$ & $\cdots$ & $\cdots$ & $\cdots$ \\
\hline G190.15-13.75South & $\leq 13.0$ & $1.0 \pm 0.1^{\dagger}$ & $\leq 4.2$ & $\leq 3.5$ & $\leq 51.0$ & $\leq 65.0$ & $\leq 1.30$ & $\cdots$ & $\geq 0.2$ & $\geq 0.2$ & $0.6 \pm 0.1$ & $\ldots$ \\
\hline G191.90-11.21North & $\leq 9.4$ & $\leq 1.4$ & $\leq 3.4$ & $\leq 2.8$ & $\leq 25.0$ & $\leq 24.0$ & $\cdots$ & $\cdots$ & $\cdots$ & $\cdots$ & $\cdots$ & $\cdots$ \\
\hline G191.90-11.21South & $5.5 \pm 3.0^{\dagger}$ & $6.9 \pm 1.3$ & $20.0 \pm 10.0$ & $3.5 \pm 1.8$ & $\leq 23.0$ & $\leq 22.0$ & $0.08 \pm 0.05$ & $5.7 \pm 4.1$ & $\geq 3.0$ & $\geq 3.1$ & $0.7 \pm 0.1$ & $4.5 \pm 0.5$ \\
\hline G192.12-10.90North & $8.8 \pm 4.6^{\dagger}$ & $3.7 \pm 0.6$ & $22.0 \pm 11.0$ & $8.1 \pm 4.1$ & $30.0 \pm 16.0$ & $79.0 \pm 40.0$ & $0.24 \pm 0.13$ & $2.7 \pm 1.9$ & $1.2 \pm 0.7$ & $0.5 \pm 0.2$ & $0.8 \pm 0.1$ & $2.1 \pm 0.2$ \\
\hline G192.12-10.90South & $\leq 8.5$ & $\leq 1.4$ & $\leq 3.4$ & $\leq 2.3$ & $6.6 \pm 4.2$ & $\leq 28.0$ & $\cdots$ & $\cdots$ & $\leq 2.1$ & $\cdots$ & $\cdots$ & $\cdots$ \\
\hline G192.12-11.10 & $\leq 8.5$ & $5.1 \pm 0.9$ & $20.0 \pm 10.0$ & $6.3 \pm 3.2$ & $\leq 25.0$ & $\leq 27.0$ & $\leq 0.17$ & $3.2 \pm 2.3$ & $\geq 2.0$ & $\geq 1.9$ & $1.4 \pm 0.2$ & $2.7 \pm 0.4$ \\
\hline G192.32-11.88North & $17.0 \pm 8.7^{\dagger}$ & $11.0 \pm 1.6$ & $34.0 \pm 17.0$ & $6.5 \pm 3.3$ & $\leq 21.0$ & $\leq 18.0$ & $0 . \overline{15} \pm 0.08$ & $5.2 \pm 3.7$ & $\geq 5.2$ & $\geq 6.1$ & $1.1 \pm 0.2$ & $4.0 \pm 0.4$ \\
\hline G192.32-11.88South & $16.0 \pm 0.8^{\dagger}$ & $9.6 \pm 1.0$ & $36.0 \pm 18.0$ & $9.5 \pm 4.8$ & $\leq 20.0$ & $37.0 \pm 19.0$ & $0.17 \pm 0.02$ & $3.8 \pm 2.7$ & $\geq 4.8$ & $2.6 \pm 1.4$ & $0.9 \pm 0.1$ & $3.0 \pm 0.2$ \\
\hline G196.92-10.37 & $\leq 11.0$ & $10.0 \pm 1.2$ & $22.0 \pm 11.0$ & $12.0 \pm 6.1$ & $\leq 28.0$ & $140.0 \pm 71.0$ & $\leq 0.11$ & $1.8 \pm 1.3$ & $\geq 3.6$ & $0.7 \pm 0.4$ & $1.5 \pm 0.2$ & $1.3 \pm 0.1$ \\
\hline G198.69-09.12North1 & $\leq 7.2$ & $1.7 \pm 0.5$ & $\geq 5.4^{\ddagger}$ & $\leq 2.2$ & $\leq 36.0$ & $\leq 45.0$ & $\leq 0.42$ & $\geq 2.5$ & $\geq 0.5$ & $\geq 0.4$ & $0.9 \pm 0.3$ & $\geq 1.1$ \\
\hline G198.69-09.12North2 & $\geq 6.1^{\ddagger}$ & $3.0 \pm 0.8$ & $\geq 18.0^{\ddagger}$ & $\geq 5.0^{\ddagger}$ & $\leq 36.0$ & $\geq 28.0^{\ddagger}$ & $\geq 0.20$ & $\leq 3.6$ & $\geq 0.8$ & $\leq 1.1$ & $1.0 \pm 0.3$ & $2 . \overline{6} \pm 0.3$ \\
\hline G198.69-09.12South & $\leq 7.0$ & $12.0 \pm 1.4$ & $\geq 18.0^{\ddagger}$ & $\geq 8.7^{\ddagger}$ & $\leq 35.0$ & $\geq 71.0^{\ddagger}$ & $\leq 0.06$ & $\leq 2.1$ & $\geq 3.4$ & $\leq 1.7$ & $1.2 \pm 0.1$ & $1.7 \pm 0.1$ \\
\hline G200.34-10.97North & $6.5 \pm 3.7^{\dagger}$ & $7.5 \pm 0.8$ & $25.0 \pm 13.0$ & $4.8 \pm 2.6$ & $\leq 28.0$ & $50.0 \pm 26.0$ & $0.09 \pm 0.05$ & $5.2 \pm 3.9$ & $\geq 2.7$ & $1.5 \pm 0.8$ & $0.8 \pm 0.1$ & $4.1 \pm 0.7$ \\
\hline G200.34-10.97South & $\leq 14.0$ & $7.0 \pm 1.2$ & $\leq 5.7$ & $\leq 4.4$ & $\leq 27.0$ & $49.0 \pm 25.0$ & $\leq 0.20$ & $\cdots$ & $\geq 2.6$ & $1.4 \pm 0.8$ & $1.0 \pm 0.2$ & $\cdots$ \\
\hline G201.52-11.08 & $\leq 17.0$ & $\leq 1.5$ & $\leq 6.4$ & $\leq 4.8$ & $\leq 26.0$ & $\leq 33.0$ & $\ldots$ & $\cdots$ & $\ldots$ & $\cdots$ & $\cdots$ & $\cdots$ \\
\hline G201.72-11.22 & $\leq 16.0$ & $3.5 \pm 0.5$ & $24.0 \pm 12.0$ & $8.0 \pm 4.1$ & $\leq 29.0$ & $61.0 \pm 31.0$ & $\leq 0.46$ & $3.0 \pm 2.1$ & $\geq 1.2$ & $0.6 \pm 0.3$ & $0.5 \pm 0.1$ & $2.2 \pm 0.3$ \\
\hline G203.21-11.20East1 & $\geq 23.0^{\ddagger}$ & $11.0 \pm 1.4$ & $\geq 70.0^{\ddagger}$ & $\geq 18.0^{\ddagger}$ & $\geq 34.0^{\ddagger}$ & $\geq 45.0^{\ddagger}$ & $\geq 0.21$ & $\leq 3.9$ & $\leq 3.2$ & $\leq 2.4$ & $1.6 \pm 0.2$ & $2.7 \pm 0.2$ \\
\hline G203.21-11.20East2 & $\geq 22.0^{\ddagger}$ & $7.4 \pm 0.9$ & $\geq 56.0^{\ddagger}$ & $\geq 12.0^{\ddagger}$ & $\leq 40.0$ & $\geq 18.0^{\ddagger}$ & $\geq 0.30$ & $\leq 4.7$ & $\geq 1.9$ & $\leq 4.1$ & $0.9 \pm 0.1$ & $3.3 \pm 0.3$ \\
\hline G203.21-11.20West 1 & $48.0 \pm 5.6$ & $16.0 \pm 0.5$ & $\geq 74.0^{\ddagger}$ & $\geq 18.0^{\ddagger}$ & $\leq 38.0$ & $\geq 37.0^{\ddagger}$ & $0 . \overline{30} \pm 0.04$ & $\leq 4.1$ & $\geq 4.2$ & $\leq 4.3$ & $1.0 \pm 0.1$ & $2.9 \pm 0.2$ \\
\hline G203.21-11.20West2 & $32.0 \pm 5.8$ & $15.0 \pm 2.0$ & $\geq 52.0^{\ddagger}$ & $\geq 13.0^{\ddagger}$ & $\geq 18.0^{\ddagger}$ & $\geq 33.0^{\ddagger}$ & $0.21 \pm 0.05$ & $\leq 4.0$ & $\leq 8.3$ & $\leq 4.5$ & $1.0 \pm 0.1$ & $3.0 \pm 0.2$ \\
\hline G204.4-11.3A2East & $38.0 \pm 9.5$ & $14.0 \pm 0.9$ & $\geq 100.0^{\ddagger}$ & $\geq 24.0^{\ddagger}$ & $\geq 14.0^{\ddagger}$ & $\geq 120.0^{\ddagger}$ & $0.27 \pm 0.07$ & $\leq 4.2$ & $\leq 10.0$ & $\leq 1.2$ & $1.0 \pm 0.1$ & $2.9 \pm 0.1$ \\
\hline G204.4-11.3A2West & $7.5 \pm 2.8^{\dagger}$ & $8.2 \pm 1.6$ & $\geq 26.0^{\ddagger}$ & $\leq 5.2$ & $\leq 44.0$ & $\geq 41.0^{\ddagger}$ & $0.09 \pm 0.04$ & $\geq 5.0$ & $\geq 1.9$ & $\leq 2.0$ & $1.7 \pm 0.3$ & $\geq 2.3$ \\
\hline G205.46-14.56Middle1 & $\leq 13.0$ & $\geq 14.0^{\ddagger}$ & $\geq 18.0^{\ddagger}$ & $\leq 4.1$ & $\leq 34.0$ & $\geq 37.0^{\ddagger}$ & $\leq 0.09$ & $\geq 4.4$ & $\geq 4.1$ & $\leq 3.8$ & $\ldots$ & $\geq 1.9$ \\
\hline G205.46-14.56Middle2 & $\leq 9.2$ & $7.9 \pm 0.6$ & $\geq 16.0^{\ddagger}$ & $\geq 8.2^{\ddagger}$ & $\leq 22.0$ & $\geq 40.0^{\ddagger}$ & $\leq 0.12$ & $\leq 2.0$ & $\geq 3.6$ & $\leq 2.0$ & $1.4 \pm 0.1$ & $1.8 \pm 0.2$ \\
\hline G205.46-14.56Middle3 & $\leq 9.9$ & $11.0 \pm 0.7$ & $\geq 16.0^{\ddagger}$ & $\geq 9.6^{\ddagger}$ & $\leq 21.0$ & $\geq 38.0^{\ddagger}$ & $\leq 0.09$ & $\leq 1.7$ & $\geq 5.2$ & $\leq 2.9$ & $1.5 \pm 0.1$ & $1.3 \pm 0.1$ \\
\hline G205.46-14.56North3 & $\geq 9.9^{\ddagger}$ & $10.0 \pm 0.6$ & $\geq 29.0^{\ddagger}$ & $\geq 10.0^{\ddagger}$ & $\leq 21.0$ & $\leq 34.0$ & $\geq 0.10$ & $\leq 2.9$ & $\geq 4.8$ & $\geq 2.9$ & $1.4 \pm 0.1$ & $2.4 \pm 0.2$ \\
\hline G205.46-14.56North2 & $\geq 5.1^{\ddagger}$ & $8.2 \pm 0.2$ & $\geq 18.0^{\ddagger}$ & $\geq 7.1^{\ddagger}$ & $\leq 22.0$ & $\geq 35.0^{\ddagger}$ & $\geq 0.06$ & $\leq 2.5$ & $\geq 3.7$ & $\leq 2.3$ & $1.7 \pm 0.1$ & $2.2 \pm 0.2$ \\
\hline G205.46-14.56North1 & $\geq 15.0^{\ddagger}$ & $15.0 \pm 0.8$ & $\geq 39.0^{\ddagger}$ & $\geq 11.0^{\ddagger}$ & $\leq 23.0$ & $\geq 29.0^{\ddagger}$ & $\geq 0.10$ & $\leq 3.5$ & $\geq 6.5$ & $\leq 5.2$ & $1.1 \pm 0.1$ & $2.6 \pm 0.2$ \\
\hline G205.46-14.56South1 & $\leq 9.4$ & $27.0 \pm 0.9$ & $\geq 19.0^{\ddagger}$ & $\geq 12.0^{\ddagger}$ & $\geq 13.0^{\ddagger}$ & $\geq 75.0^{\ddagger}$ & $\leq 0.04$ & $\leq 1.6$ & $\leq 20.8$ & $\leq 3.6$ & $2.4 \pm 0.1$ & $1.3 \pm 0.1$ \\
\hline G205.46-14.56South2 & $\leq 9.0$ & $11.0 \pm 0.5$ & $\geq 20.0^{\ddagger}$ & $\geq 10.0^{\ddagger}$ & $\geq 18.0^{\ddagger}$ & $\geq 64.0^{\ddagger}$ & $\leq 0.08$ & $\leq 2.0$ & $\leq 6.1$ & $\leq 1.7$ & $0.9 \pm 0.1$ & $1.6 \pm 0.1$ \\
\hline G205.46-14.56South3 & $11 . \overline{0} \pm 0.8^{\dagger}$ & $9.8 \pm 0.6$ & $\geq 35.0^{\ddagger}$ & $\geq 11.0^{\ddagger}$ & $\leq 22.0$ & $\geq 29.0^{\ddagger}$ & $0 . \overline{11} \pm 0.01$ & $\leq 3.2$ & $\geq 4.5$ & $\leq 3.4$ & $1.2 \pm 0.1$ & $2.5 \pm 0.2$ \\
\hline G206.12-15.76 & $37.0 \pm 14.0$ & $\cdots$ & $\geq 110.0^{\ddagger}$ & $\geq 23.0^{\ddagger}$ & $\cdots$ & $\cdots$ & $\cdots$ & $\leq 4.8$ & $\cdots$ & $\ldots$ & $\cdots$ & $3.0 \pm 0.3$ \\
\hline G206.21-16.17North & $25.0 \pm 13.0^{\dagger}$ & $\ldots$ & $21.0 \pm 11.0$ & $\leq 8.8$ & $\ldots$ & $\ldots$ & $\ldots$ & $\geq 2.4$ & $\ldots$ & $\ldots$ & $\ldots$ & $\geq 1.0$ \\
\hline G206.69-16.60North & $\leq 16.0$ & $4.4 \pm 0.5$ & $21.0 \pm 11.0$ & $\leq 5.0$ & $\leq 18.0$ & $18.0 \pm 9.7$ & $\leq 0.36$ & $\geq 4.2$ & $\geq 2.4$ & $2.4 \pm 1.4$ & $0.6 \pm 0.1$ & $\geq 1.8$ \\
\hline G206.69-16.60South & $\leq 15.0$ & $6.8 \pm 1.0$ & $16.0 \pm 8.1$ & $\leq 5.3$ & $\leq 17.0$ & $11.0 \pm 6.0$ & $\leq 0.22$ & $\geq 3.0$ & $\geq 4.0$ & $6.2 \pm 3.5$ & $0.9 \pm 0.1$ & $\geq 1.3$ \\
\hline
\end{tabular}


Table 5 (continued)

\begin{tabular}{|c|c|c|c|c|c|c|c|c|c|c|c|c|}
\hline SCUBA-2 core & $\begin{array}{c}N\left(\mathrm{~N}_{2} \mathrm{D}^{+}\right) \\
\left(10^{11} \mathrm{~cm}^{-2}\right) \\
(2)\end{array}$ & $\begin{array}{c}N\left(\mathrm{~N}_{2} \mathrm{H}^{+}\right) \\
\left(10^{12} \mathrm{~cm}^{-2}\right) \\
(3)\end{array}$ & $\begin{array}{c}N(\mathrm{DNC}) \\
\left(10^{11} \mathrm{~cm}^{-2}\right) \\
(4)\end{array}$ & $\begin{array}{c}N\left(\mathrm{HN}^{13} \mathrm{C}\right) \\
\left(10^{11} \mathrm{~cm}^{-2}\right) \\
(5)\end{array}$ & $\begin{array}{c}N(\mathrm{CCS}) \\
\left(10^{11} \mathrm{~cm}^{-2}\right) \\
(6)\end{array}$ & $\begin{array}{c}N\left(\mathrm{HC}_{3} \mathrm{~N}\right) \\
\left(10^{11} \mathrm{~cm}^{-2}\right) \\
(7)\end{array}$ & $\frac{N\left(\mathrm{~N}_{2} \mathrm{D}^{+}\right)}{N\left(\mathrm{~N}_{2} \mathrm{H}^{+}\right)}$ & $\frac{N(\mathrm{DNC})}{N\left(\mathrm{HN}^{13} \mathrm{C}\right)}$ & $\frac{N\left(\mathrm{~N}_{2} \mathrm{H}^{+}\right)}{N(\mathrm{CCS})}$ & $\frac{N\left(\mathrm{~N}_{2} \mathrm{H}^{+}\right)}{N\left(\mathrm{HC}_{3} \mathrm{~N}\right)}$ & (12) & $\frac{\int T(\mathrm{DNC}) d v}{\int T\left(\mathrm{HN}^{13} \mathrm{C}\right) d v}$ \\
\hline G206.93-16.61East1 & $\leq 14.0$ & $18.0 \pm 0.1$ & $41.0 \pm 21.0$ & $13.0 \pm 6.6$ & $\leq 15.0$ & $22.0 \pm 11.0$ & $\leq 0.08$ & $3.2 \pm 2.3$ & $\geq 12.0$ & $8.2 \pm 4.1$ & $1.3 \pm 0.1$ & $2.8 \pm 0.3$ \\
\hline G206.93-16.61East2 & $\leq 13.0$ & $2.7 \pm 0.1^{\dagger}$ & $7.8 \pm 4.0$ & $5.1 \pm 2.7$ & $\leq 17.0$ & $\leq 15.0$ & $\leq 0.48$ & $1.5 \pm 1.1$ & $\geq 1.6$ & $\geq 1.8$ & $1.0 \pm 0.1$ & $1.1 \pm 0.2$ \\
\hline G206.93-16.61West1 & $\leq 14.0$ & $9.4 \pm 0.2$ & $17.0 \pm 8.6$ & $17.0 \pm 8.6$ & $\leq 17.0$ & $73.0 \pm 37.0$ & $\leq 0.15$ & $1.0 \pm 0.7$ & $\geq 5.5$ & $1.3 \pm 0.7$ & $1.4 \pm 0.1$ & $0.8 \pm 0.1$ \\
\hline G206.93-16.61West3 & $7 . \overline{7} \pm 4.4^{\dagger}$ & $22.0 \pm 1.1$ & $48.0 \pm 24.0$ & $28.0 \pm 14.0$ & $\leq 15.0$ & $180.0 \pm 90.0$ & $0.04 \pm 0.02$ & $1.7 \pm 1.2$ & $\geq 14.7$ & $1.2 \pm 0.6$ & $1.1 \pm 0.1$ & $1.3 \pm 0.1$ \\
\hline G206.93-16.61West4 & $8.5 \pm 1.7^{\dagger}$ & $5.3 \pm 0.7$ & $26.0 \pm 13.0$ & $5.1 \pm 2.7$ & $\leq 16.0$ & $\leq 15.0$ & $0.16 \pm 0.04$ & $5.1 \pm 3.7$ & $\geq 3.3$ & $\geq 3.5$ & $1.1 \pm 0.1$ & $3.8 \pm 0.7$ \\
\hline G206.93-16.61West5 & $12.0 \pm 0.7^{\dagger}$ & $8.2 \pm 0.8$ & $22.0 \pm 11.0$ & $\leq 3.9$ & $\leq 14.0$ & $\leq 15.0$ & $0.15 \pm 0.02$ & $\geq 5.6$ & $\geq 5.9$ & $\geq 5.5$ & $1.4 \pm 0.1$ & $\geq 2.2$ \\
\hline G206.93-16.61West6 & $\leq 14.0$ & $\leq 1.2$ & $\leq 5.0$ & $\leq 4.0$ & $\leq 16.0$ & $\leq 14.0$ & $\cdots$ & $\cdots$ & $\cdots$ & $\ldots$ & $\cdots$ & $\cdots$ \\
\hline G206.93-16.61West6 & $\leq 14.0$ & $6.0 \pm 0.5$ & $16.0 \pm 8.2$ & $6.0 \pm 3.1$ & $\leq 16.0$ & $\leq 14.0$ & $\leq 0.23$ & $2.7 \pm 1.9$ & $\geq 3.8$ & $\geq 4.3$ & $1.0 \pm 0.1$ & $2.3 \pm 0.4$ \\
\hline G207.36-19.82North1 & $\leq 16.0$ & $9.9 \pm 0.1$ & $\geq 22.0^{\ddagger}$ & $\geq 11.0^{\ddagger}$ & $\leq 28.0$ & $\geq 63.0^{\ddagger}$ & $\leq 0.16$ & $\leq 2.0$ & $\geq 3.5$ & $\leq 1.6$ & $2.3 \pm 0.1$ & $1.8 \pm 0.3$ \\
\hline G207.36-19.82North2 & $19.0 \pm 3.5^{\dagger}$ & $8.6 \pm 0.8$ & $\geq 50.0^{\ddagger}$ & $\geq 7.4^{\ddagger}$ & $\leq 28.0$ & $\geq 31.0^{\ddagger}$ & $0.22 \pm 0.05$ & $\leq 6.8$ & $\geq 3.1$ & $\leq 2.8$ & $0.9 \pm 0.1$ & $4.9 \pm 0.6$ \\
\hline G207.36-19.82North3 & $\leq 14.0$ & $7.0 \pm 1.0$ & $\geq 32.0^{\ddagger}$ & $\leq 4.7$ & $\leq 29.0$ & $\geq 6.9^{\ddagger}$ & $\leq 0.20$ & $\geq 6.8$ & $\geq 2.4$ & $\leq 10.1$ & $1.4 \pm 0.2$ & $\geq 2.6$ \\
\hline G207.36-19.82North4 & $\leq 14.0$ & $7.1 \pm 1.2$ & $\geq 24.0^{\ddagger}$ & $\leq 4.6$ & $\leq 30.0$ & $\leq 36.0$ & $\leq 0.20$ & $\geq 5.2$ & $\geq 2.4$ & $\geq 2.0$ & $1.6 \pm 0.3$ & $\geq 2.3$ \\
\hline G207.36-19.82South & $6.3 \pm 1.9^{\dagger}$ & $5.7 \pm 0.8$ & $\geq 23.0^{\ddagger}$ & $\leq 4.6$ & $\leq 28.0$ & $\geq 23.0^{\ddagger}$ & $0.11 \pm 0.04$ & $\geq 5.0$ & $\geq 2.0$ & $\leq 2.5$ & $0.7 \pm 0.1$ & $\geq 2.4$ \\
\hline G207.3-19.8A2North1 & $\leq 15.0$ & $\geq 1.8^{\ddagger}$ & $\leq 5.5$ & $\leq 4.6$ & $\leq 29.0$ & $\geq 5.2^{\ddagger}$ & $\leq 0.83$ & $\ldots$ & $\geq 0.6$ & $\leq 3.5$ & $\ldots$ & $\ldots$ \\
\hline G207.3-19.8A2North2 & $\leq 16.0$ & $1.8 \pm 0.1^{\dagger}$ & $\geq 9.8^{\ddagger}$ & $\leq 4.4$ & $\leq 30.0$ & $\leq 35.0$ & $\leq 0.89$ & $\geq 2.2$ & $\geq 0.6$ & $\geq 0.5$ & $0.8 \pm 0.1$ & $\geq 0.8$ \\
\hline G207.3-19.8A2North3 & $\leq 15.0$ & $\leq 1.2$ & $\leq 5.9$ & $\leq 4.2$ & $\leq 28.0$ & $\leq 32.0$ & $\cdots$ & $\cdots$ & $\cdots$ & $\cdots$ & $\cdots$ & $\cdots$ \\
\hline G207.3-19.8A2South & $\leq 21.0$ & $2.5 \pm 0.5$ & $\leq 7.6$ & $\leq 5.8$ & $\leq 31.0$ & $\leq 30.0$ & $\leq 0.84$ & $\ldots$ & $\geq 0.8$ & $\geq 0.8$ & $0.9 \pm 0.2$ & $\ldots$ \\
\hline G208.68-19.20North1 & $\leq 14.0$ & $18.0 \pm 0.8$ & $46.0 \pm 23.0$ & $18.0 \pm 9.1$ & $\leq 14.0$ & $140.0 \pm 70.0$ & $\leq 0.08$ & $2.6 \pm 1.8$ & $\geq 12.9$ & $1.3 \pm 0.6$ & $1.1 \pm 0.1$ & $2.0 \pm 0.1$ \\
\hline G208.68-19.20North2 & $75.0 \pm 7.1$ & $67.0 \pm 1.2$ & $75.0 \pm 38.0$ & $24.0 \pm 12.0$ & $\leq 15.0$ & $60.0 \pm 30.0$ & $0.11 \pm 0.01$ & $3.1 \pm 2.2$ & $\geq 44.7$ & $11.2 \pm 5.6$ & $0.6 \pm 0.1$ & $2.1 \pm 0.1$ \\
\hline G208.68-19.20North3 & $36.0 \pm 6.9$ & $58.0 \pm 1.4$ & $56.0 \pm 28.0$ & $21.0 \pm 11.0$ & $\leq 14.0$ & $100.0 \pm 50.0$ & $0.06 \pm 0.01$ & $2.7 \pm 1.9$ & $\geq 41.4$ & $5.8 \pm 2.9$ & $1.1 \pm 0.1$ & $2.1 \pm 0.1$ \\
\hline G208.68-19.20South & $16.0 \pm 8.3^{\dagger}$ & $25.0 \pm 0.1$ & $31.0 \pm 16.0$ & $9.6 \pm 4.9$ & $\leq 14.0$ & $57.0 \pm 29.0$ & $0.06 \pm 0.03$ & $3.2 \pm 2.3$ & $\geq 17.9$ & $4.4 \pm 2.2$ & $1.5 \pm 0.1$ & $2.5 \pm 0.3$ \\
\hline G208.89-20.04East & $\geq 22.0^{\ddagger}$ & $19.0 \pm 1.5$ & $\geq 57.0^{\ddagger}$ & $\geq 22.0^{\ddagger}$ & $\leq 34.0$ & $\geq 84.0^{\ddagger}$ & $\geq 0.12$ & $\leq 2.6$ & $\geq 5.6$ & $\leq 2.3$ & $0.7 \pm 0.1$ & $1.9 \pm 0.1$ \\
\hline G209.05-19.73North & $8.9 \pm 1.8^{\dagger}$ & $3.3 \pm 0.1$ & $17.0 \pm 8.6$ & $3.0 \pm 1.6$ & $\leq 26.0$ & $\leq 25.0$ & $0.27 \pm 0.06$ & $5.7 \pm 4.2$ & $\geq 1.3$ & $\geq 1.3$ & $0.6 \pm 0.1$ & $4.5 \pm 1.1$ \\
\hline G209.05-19.73South & $7.9 \pm 4.3^{\dagger}$ & $3.4 \pm 0.2$ & $29.0 \pm 15.0$ & $4.9 \pm 2.6$ & $\leq 28.0$ & $30.0 \pm 16.0$ & $0.23 \pm 0.13$ & $5.9 \pm 4.4$ & $\geq 1.2$ & $1.1 \pm 0.6$ & $0.7 \pm 0.1$ & $4.8 \pm 0.8$ \\
\hline G209.29-19.65North1 & $8.5 \pm 0.9$ & $14.0 \pm 7.0^{\dagger}$ & $13.0 \pm 6.7$ & $6.6 \pm 3.4$ & $\leq 16.0$ & $\leq 14.0$ & $0.06 \pm 0.03$ & $2.0 \pm 1.4$ & $\geq 8.8$ & $\geq 10.0$ & $\ldots$ & $1.7 \pm 0.3$ \\
\hline G209.29-19.65North2 & $\leq 11.0$ & $3.7 \pm 0.1$ & $\leq 4.1$ & $\leq 3.4$ & $\leq 16.0$ & $\leq 16.0$ & $\leq 0.30$ & $\cdots$ & $\geq 2.3$ & $\geq 2.3$ & $0.4 \pm 0.1$ & $\cdots$ \\
\hline G209.29-19.65North2 & $9.0 \pm 1.8^{\dagger}$ & $5.1 \pm 0.1^{\dagger}$ & $17.0 \pm 8.6$ & $\leq 3.3$ & $\leq 16.0$ & $\leq 16.0$ & $0.18 \pm 0.04$ & $\geq 5.2$ & $\geq 3.2$ & $\geq 3.2$ & $0.4 \pm 0.1$ & $\geq 2.0$ \\
\hline G209.29-19.65North3 & $\leq 11.0$ & $4.1 \pm 0.6$ & $\leq 3.8$ & $6.2 \pm 3.2$ & $\leq 17.0$ & $\leq 15.0$ & $\leq 0.27$ & $\leq 0.6$ & $\geq 2.4$ & $\geq 2.7$ & $1.1 \pm 0.2$ & $\leq 1.5$ \\
\hline G209.29-19.65South1 & $18.0 \pm 3.3^{\dagger}$ & $17.0 \pm 1.0$ & $37.0 \pm 19.0$ & $\leq 4.0$ & $\leq 14.0$ & $\leq 15.0$ & $0.11 \pm 0.02$ & $\geq 9.2$ & $\geq 12.1$ & $\geq 11.3$ & $2.4 \pm 0.1$ & $\geq 3.8$ \\
\hline G209.29-19.65South2 & $\leq 12.0$ & $15.0 \pm 1.2$ & $30.0 \pm 15.0$ & $8.7 \pm 4.5$ & $\leq 14.0$ & $\leq 15.0$ & $\leq 0.08$ & $3.4 \pm 2.5$ & $\geq 10.7$ & $\geq 10.0$ & $1.9 \pm 0.2$ & $2.1 \pm 0.3$ \\
\hline G209.29-19.65South2 & $14.0 \pm 7.4^{\dagger}$ & $9.9 \pm 1.2$ & $5.6 \pm 2.9$ & $8.0 \pm 4.1$ & $\leq 15.0$ & $\leq 15.0$ & $0.14 \pm 0.08$ & $0.7 \pm 0.5$ & $\geq 6.6$ & $\geq 6.6$ & $0.6 \pm 0.1$ & $0.6 \pm 0.1$ \\
\hline G209.29-19.65South3 & $\leq 11.0$ & $7.3 \pm 0.8$ & $13.0 \pm 6.6$ & $\leq 3.7$ & $\leq 14.0$ & $\leq 15.0$ & $\leq 0.15$ & $\geq 3.5$ & $\geq 5.2$ & $\geq 4.9$ & $1.1 \pm 0.1$ & $\geq 1.6$ \\
\hline G209.55-19.68North1 & $\leq 11.0$ & $22.0 \pm 1.2$ & $24.0 \pm 12.0$ & $12.0 \pm 6.1$ & $12.0 \pm 6.4$ & $\leq 22.0$ & $\leq 0.05$ & $2.0 \pm 1.4$ & $18.3 \pm 9.8$ & $\geq 10.0$ & $1.6 \pm 0.1$ & $1.6 \pm 0.2$ \\
\hline G209.55-19.68North2 & $9.9 \pm 5.3^{\dagger}$ & $4.4 \pm 0.1$ & $22.0 \pm 11.0$ & $5.4 \pm 2.8$ & $\leq 19.0$ & $\leq 23.0$ & $0.23 \pm 0.12$ & $4.1 \pm 2.9$ & $\geq 2.3$ & $\geq 1.9$ & $0.5 \pm 0.1$ & $2.9 \pm 0.3$ \\
\hline G209.55-19.68North3 & $9.4 \pm 1.9^{\dagger}$ & $9.8 \pm 0.8$ & $33.0 \pm 17.0$ & $14.0 \pm 7.1$ & $14.0 \pm 7.5$ & $36.0 \pm 19.0$ & $0.10 \pm 0.02$ & $2.4 \pm 1.7$ & $7.0 \pm 3.8$ & $2 . \overline{7} \pm 1.5$ & $0.9 \pm 0.1$ & $1.7 \pm 0.2$ \\
\hline G209.55-19.68South1 & $10.0 \pm 1.7^{\dagger}$ & $16.0 \pm 1.0$ & $33.0 \pm 17.0$ & $11.0 \pm 5.6$ & $\leq 21.0$ & $41.0 \pm 21.0$ & $0.06 \pm 0.01$ & $3.0 \pm 2.2$ & $\geq 7.6$ & $3.9 \pm 2.0$ & $1.3 \pm 0.1$ & $2.6 \pm 0.2$ \\
\hline G209.55-19.68South3 & $12.0 \pm 1.5^{\dagger}$ & $15.0 \pm 0.7$ & $41.0 \pm 21.0$ & $14.0 \pm 7.1$ & $\leq 21.0$ & $57.0 \pm 29.0$ & $0.08 \pm 0.01$ & $2.9 \pm 2.1$ & $\geq 7.1$ & $2.6 \pm 1.3$ & $0.8 \pm 0.1$ & $2.2 \pm 0.2$ \\
\hline G209.77-19.40East1 & $8.9 \pm 1.5^{\dagger}$ & $19.0 \pm 0.8$ & $30.0 \pm 15.0$ & $17.0 \pm 8.6$ & $12.0 \pm 6.6$ & $54.0 \pm 27.0$ & $0.05 \pm 0.01$ & $1.8 \pm 1.3$ & $15.8 \pm 8.7$ & $3.5 \pm 1.8$ & $0.6 \pm 0.1$ & $1.5 \pm 0.1$ \\
\hline G209.77-19.40East2 & $\leq 13.0$ & $9.1 \pm 0.7$ & $35.0 \pm 18.0$ & $14.0 \pm 7.1$ & $\leq 19.0$ & $49.0 \pm 25.0$ & $\leq 0.14$ & $2.5 \pm 1.8$ & $\geq 4.8$ & $1.9 \pm 1.0$ & $0.9 \pm 0.1$ & $1.9 \pm 0.2$ \\
\hline
\end{tabular}


Table 5 (continued)

\begin{tabular}{|c|c|c|c|c|c|c|c|c|c|c|c|c|}
\hline SCUBA-2 core & $\begin{array}{c}N\left(\mathrm{~N}_{2} \mathrm{D}^{+}\right) \\
\left(10^{11} \mathrm{~cm}^{-2}\right) \\
(2)\end{array}$ & $\begin{array}{c}N\left(\mathrm{~N}_{2} \mathrm{H}^{+}\right) \\
\left(10^{12} \mathrm{~cm}^{-2}\right) \\
(3)\end{array}$ & $\begin{array}{c}N(\mathrm{DNC}) \\
\left(10^{11} \mathrm{~cm}^{-2}\right) \\
(4)\end{array}$ & $\begin{array}{c}N\left(\mathrm{HN}^{13} \mathrm{C}\right) \\
\left(10^{11} \mathrm{~cm}^{-2}\right) \\
(5)\end{array}$ & $\begin{array}{c}N(\mathrm{CCS}) \\
\left(10^{11} \mathrm{~cm}^{-2}\right) \\
(6)\end{array}$ & $\begin{array}{c}N\left(\mathrm{HC}_{3} \mathrm{~N}\right) \\
\left(10^{11} \mathrm{~cm}^{-2}\right) \\
(7)\end{array}$ & $\frac{N\left(\mathrm{~N}_{2} \mathrm{D}^{+}\right)}{N\left(\mathrm{~N}_{2} \mathrm{H}^{+}\right)}$ & $\frac{N(\mathrm{DNC})}{N\left(\mathrm{HN}^{13} \mathrm{C}\right)}$ & $\frac{N\left(\mathrm{~N}_{2} \mathrm{H}^{+}\right)}{N(\mathrm{CCS})}$ & $\frac{N\left(\mathrm{~N}_{2} \mathrm{H}^{+}\right)}{N\left(\mathrm{HC}_{3} \mathrm{~N}\right)}$ & (12) & $\frac{\int T(\mathrm{DNC}) d v}{\int T\left(\mathrm{HN}^{13} \mathrm{C}\right) d v}$ \\
\hline G209.77-19.40East3 & $9.6 \pm 1.1^{\dagger}$ & $3.8 \pm 0.4$ & $33.0 \pm 17.0$ & $7.0 \pm 3.6$ & $\leq 20.0$ & $18.0 \pm 9.5$ & $0.25 \pm 0.04$ & $4.7 \pm 3.4$ & $\geq 1.9$ & $2.1 \pm 1.1$ & $0.3 \pm 0.1$ & $4.1 \pm 0.7$ \\
\hline G209.77-19.40East3 & $\leq 12.0$ & $1.9 \pm 0.1^{\dagger}$ & $7.4 \pm 3.8$ & $4.0 \pm 2.1$ & $\leq 20.0$ & $14.0 \pm 7.5$ & $\leq 0.63$ & $1.9 \pm 1.4$ & $\geq 0.9$ & $1.4 \pm 0.7$ & $0.5 \pm 0.1$ & $1.9 \pm 0.5$ \\
\hline G209.77-19.40West & $\leq 12.0$ & $5.8 \pm 0.5$ & $12.0 \pm 6.1$ & $5.7 \pm 3.0$ & $7.6 \pm 4.4$ & $16.0 \pm 8.6$ & $\leq 0.21$ & $2.1 \pm 1.5$ & $7.6 \pm 4.5$ & $3.6 \pm 2.0$ & $0.3 \pm 0.1$ & $2.1 \pm 0.3$ \\
\hline G209.77-19.61East & $\leq 12.0$ & $2.6 \pm 1.3^{\dagger}$ & $2.4 \pm 1.3$ & $\leq 4.2$ & $\leq 22.0$ & $19.0 \pm 10.0$ & $\leq 0.46$ & $\geq 0.6$ & $\geq 1.2$ & $1.4 \pm 1.0$ & $\ldots$ & $\geq 0.4$ \\
\hline G209.77-19.61West & $\leq 11.0$ & $3.2 \pm 0.2^{\dagger}$ & $19.0 \pm 9.6$ & $\leq 4.1$ & $\leq 23.0$ & $18.0 \pm 9.9$ & $\leq 0.34$ & $\geq 4.6$ & $\geq 1.4$ & $1.8 \pm 1.0$ & $0.9 \pm 0.1$ & $\geq 1.9$ \\
\hline G209.79-19.80East & $\leq 12.0$ & $2.9 \pm 0.5$ & $7.0 \pm 3.6$ & $\leq 3.8$ & $\leq 22.0$ & $20.0 \pm 11.0$ & $\leq 0.41$ & $\geq 1.8$ & $\geq 1.3$ & $1.4 \pm 0.8$ & $0.7 \pm 0.1$ & $\geq 0.7$ \\
\hline G209.79-19.80West & $9.8 \pm 1.4^{\dagger}$ & $8.4 \pm 0.8$ & $50.0 \pm 25.0$ & $13.0 \pm 6.6$ & $\leq 23.0$ & $38.0 \pm 20.0$ & $0 . \overline{12} \pm 0.02$ & $3.8 \pm 2.7$ & $\geq 3.7$ & $2.2 \pm 1.2$ & $1.0 \pm 0.1$ & $2.9 \pm 0.2$ \\
\hline G209.79-19.80West & $\leq 12.0$ & $\leq 1.2$ & $\leq 3.6$ & $\leq 3.8$ & $\leq 23.0$ & $\leq 24.0$ & $\cdots$ & $\cdots$ & $\cdots$ & $\cdots$ & $\cdots$ & $\cdots$ \\
\hline G209.94-19.52North & $52.0 \pm 10.0$ & $16.0 \pm 1.0$ & $100.0 \pm 50.0$ & $28.0 \pm 14.0$ & $7.7 \pm 4.3$ & $74.0 \pm 37.0$ & $0.33 \pm 0.07$ & $3.6 \pm 2.5$ & $20.8 \pm 11.7$ & $2.2 \pm 1.1$ & $1.0 \pm 0.1$ & $2.3 \pm 0.1$ \\
\hline G209.94-19.52South1 & $17.0 \pm 8.9^{\dagger}$ & $7.1 \pm 1.0$ & $76.0 \pm 38.0$ & $12.0 \pm 6.0$ & $\leq 19.0$ & $16.0 \pm 8.5$ & $0.24 \pm 0.13$ & $6.3 \pm 4.5$ & $\geq 3.7$ & $4.4 \pm 2.4$ & $0.4 \pm 0.1$ & $3.1 \pm 0.2$ \\
\hline G209.94-19.52South1 & $16.0 \pm 1.3^{\dagger}$ & $6.6 \pm 0.5$ & $57.0 \pm 29.0$ & $14.0 \pm 7.0$ & $\leq 18.0$ & $14.0 \pm 7.5$ & $0.24 \pm 0.03$ & $4.1 \pm 2.9$ & $\geq 3.7$ & $4.7 \pm 2.6$ & $0.6 \pm 0.1$ & $1.9 \pm 0.1$ \\
\hline G209.94-19.52South2 & $\leq 12.0$ & $7.6 \pm 0.7$ & $26.0 \pm 13.0$ & $9.5 \pm 4.8$ & $\leq 17.0$ & $25.0 \pm 13.0$ & $\leq 0.16$ & $2.7 \pm 1.9$ & $\geq 4.5$ & $3.0 \pm 1.6$ & $1.0 \pm 0.1$ & $2.2 \pm 0.2$ \\
\hline G210.37-19.53North & $\leq 13.0$ & $5.8 \pm 0.4$ & $23.0 \pm 12.0$ & $3.0 \pm 1.6$ & $\leq 21.0$ & $15.0 \pm 8.0$ & $\leq 0.22$ & $7.7 \pm 5.7$ & $\geq 2.8$ & $3.9 \pm 2.1$ & $0.4 \pm 0.1$ & $3.1 \pm 0.7$ \\
\hline G210.37-19.53South & $9.5 \pm 1.6^{\dagger}$ & $7.1 \pm 0.7$ & $40.0 \pm 20.0$ & $15.0 \pm 7.6$ & $\leq 21.0$ & $100.0 \pm 50.0$ & $0.13 \pm 0.03$ & $2.7 \pm 1.9$ & $\geq 3.4$ & $0.7 \pm 0.4$ & $1.3 \pm 0.1$ & $1.9 \pm 0.2$ \\
\hline G210.49-19.79East1 & $\leq 14.0$ & $17.0 \pm 0.3$ & $\geq 29.0^{\ddagger}$ & $\geq 14.0^{\ddagger}$ & $\geq 16.0^{\ddagger}$ & $\geq 120.0^{\ddagger}$ & $\leq 0.08$ & $\leq 2.1$ & $\leq 10.6$ & $\leq 1.4$ & $1.5 \pm 0.1$ & $1.7 \pm 0.2$ \\
\hline G210.49-19.79East2 & $9 . \overline{2} \pm 1.1^{\dagger}$ & $5.4 \pm 0.6$ & $\geq 23.0^{\ddagger}$ & $\geq 6.1^{\ddagger}$ & $\leq 28.0$ & $\geq 11.0^{\ddagger}$ & $0 . \overline{17} \pm 0.03$ & $\leq 3.8$ & $\geq 1.9$ & $\leq 4.9$ & $0.6 \pm 0.1$ & $1.9 \pm 0.3$ \\
\hline G210.49-19.79East2 & $\leq 13.0$ & $17.0 \pm 0.8$ & $\geq 28.0^{\ddagger}$ & $\geq 15.0^{\ddagger}$ & $\leq 27.0$ & $\geq 72.0^{\ddagger}$ & $\leq 0.08$ & $\leq 1.9$ & $\geq 6.3$ & $\leq 2.4$ & $1.5 \pm 0.1$ & $1.5 \pm 0.1$ \\
\hline G210.49-19.79West & $20.0 \pm 2.2^{\dagger}$ & $18.0 \pm 1.1$ & $\geq 52.0^{\ddagger}$ & $\geq 18.0^{\ddagger}$ & $\leq 28.0$ & $\geq 57.0^{\ddagger}$ & $0.11 \pm 0.01$ & $\leq 2.9$ & $\geq 6.4$ & $\leq 3.2$ & $1.2 \pm 0.1$ & $2.2 \pm 0.1$ \\
\hline G210.82-19.47North1 & $23.0 \pm 1.4^{\dagger}$ & $7.1 \pm 0.5$ & $40.0 \pm 20.0$ & $10.0 \pm 5.1$ & $\leq 14.0$ & $\leq 18.0$ & $0.32 \pm 0.03$ & $4.0 \pm 2.9$ & $\geq 5.1$ & $\geq 3.9$ & $0.7 \pm 0.1$ & $2.9 \pm 0.3$ \\
\hline G210.82-19.47North2 & $12.0 \pm 1.0^{\dagger}$ & $6.1 \pm 0.1$ & $48.0 \pm 24.0$ & $9.0 \pm 4.6$ & $\leq 11.0$ & $16.0 \pm 8.2$ & $0.20 \pm 0.02$ & $5.3 \pm 3.8$ & $\geq 5.5$ & $3.8 \pm 2.0$ & $0.5 \pm 0.1$ & $3.8 \pm 0.4$ \\
\hline G210.97-19.33North & $\leq 11.0$ & $\leq 0.8$ & $\leq 3.4$ & $\leq 3.9$ & $\leq 18.0$ & $\leq 20.0$ & $\ldots$ & $\ldots$ & $\ldots$ & $\cdots$ & $\cdots$ & $\cdots$ \\
\hline G210.97-19.33South1 & $\leq 26.0$ & $4.6 \pm 0.7$ & $\leq 8.8$ & $\leq 7.4$ & $\geq 21.0^{\ddagger}$ & $\geq 45.0^{\ddagger}$ & $\leq 0.56$ & $\cdots$ & $\leq 2.2$ & $\leq 1.0$ & $0.7 \pm 0.1$ & $\cdots$ \\
\hline G210.97-19.33South2 & $\leq 24.0$ & $\geq 3.3^{\ddagger}$ & $\geq 6.1^{\ddagger}$ & $\geq 5.2^{\ddagger}$ & $\geq 14.0^{\ddagger}$ & $\geq 53.0^{\ddagger}$ & $\leq 0.73$ & $\leq 1.2$ & $\leq 2.4$ & $\leq 0.6$ & $\ldots$ & $1.4 \pm 0.4$ \\
\hline G210.97-19.33South2 & $\leq 24.0$ & $2.0 \pm 0.1$ & $\geq 33.0^{\ddagger}$ & $\leq 8.0$ & $\leq 22.0$ & $\geq 16.0^{\ddagger}$ & $\leq 1.20$ & $\geq 4.1$ & $\geq 0.9$ & $\leq 1.2$ & $0.8 \pm 0.1$ & $\geq 1.7$ \\
\hline G211.01-19.54North & $51.0 \pm 26.0^{\dagger}$ & $19.0 \pm 0.6$ & $59.0 \pm 30.0$ & $22.0 \pm 11.0$ & $56.0 \pm 28.0$ & $200.0 \pm 100.0$ & $0 . \overline{27} \pm 0.14$ & $2 . \overline{7} \pm 1.9$ & $3.4 \pm 1.7$ & $0 . \overline{9} \pm 0.5$ & $1.5 \pm 0.1$ & $1.9 \pm 0.1$ \\
\hline G211.01-19.54South & $27.0 \pm 7.5$ & $16.0 \pm 0.6$ & $42.0 \pm 21.0$ & $20.0 \pm 10.0$ & $45.0 \pm 23.0$ & $110.0 \pm 55.0$ & $0.17 \pm 0.05$ & $2.1 \pm 1.5$ & $3.6 \pm 1.8$ & $1.5 \pm 0.7$ & $1.3 \pm 0.1$ & $1.6 \pm 0.1$ \\
\hline G211.16-19.33North1 & $\leq 10.0$ & $7.1 \pm 0.7$ & $\geq 13.0^{\ddagger}$ & $\geq 9.2^{\ddagger}$ & $\geq 14.0^{\ddagger}$ & $\geq 89.0^{\ddagger}$ & $\leq 0.14$ & $\leq 1.4$ & $\leq 5.1$ & $\leq 0.8$ & $0.8 \pm 0.1$ & $1.1 \pm 0.1$ \\
\hline G211.16-19.33North2 & $10.0 \pm 4.4$ & $10.0 \pm 0.7$ & $\geq 40.0^{\ddagger}$ & $\geq 16.0^{\ddagger}$ & $\geq 12.0^{\ddagger}$ & $\geq 75.0^{\ddagger}$ & $0.10 \pm 0.04$ & $\leq 2.5$ & $\leq 8.3$ & $\leq 1.3$ & $0.9 \pm 0.1$ & $1.9 \pm 0.1$ \\
\hline G211.16-19.33North3 & $22.0 \pm 4.4$ & $9.3 \pm 0.7$ & $\geq 53.0^{\ddagger}$ & $\geq 15.0^{\ddagger}$ & $\geq 9.3^{\ddagger}$ & $\geq 38.0^{\ddagger}$ & $0.24 \pm 0.05$ & $\leq 3.5$ & $\leq 10.0$ & $\leq 2.4$ & $0.6 \pm 0.1$ & $2.3 \pm 0.1$ \\
\hline G211.16-19.33North4 & $12.0 \pm 3.8$ & $\geq 6.2^{\ddagger}$ & $\geq 41.0^{\ddagger}$ & $\geq 18.0^{\ddagger}$ & $\leq 18.0$ & $\geq 40.0^{\ddagger}$ & $\leq 0.19$ & $\leq 2.3$ & $\geq 3.4$ & $\leq 1.6$ & $\cdots$ & $2.0 \pm 0.1$ \\
\hline G211.16-19.33North5 & $31.0 \pm 5.4$ & $10.0 \pm 0.5$ & $\geq 55.0^{\ddagger}$ & $\geq 17.0^{\ddagger}$ & $\leq 17.0$ & $\geq 38.0^{\ddagger}$ & $0 . \overline{31} \pm 0.06$ & $\leq 3.2$ & $\geq 5.9$ & $\leq 2.6$ & $0.9 \pm 0.1$ & $2.2 \pm 0.1$ \\
\hline G211.16-19.33South & $\leq 12.0$ & $0.7 \pm 0.1^{\dagger}$ & $\leq 4.0$ & $\leq 3.8$ & $\leq 18.0$ & $\leq 22.0$ & $\leq 1.71$ & $\ldots$ & $\geq 0.4$ & $\geq 0.3$ & $0.9 \pm 0.2$ & $\cdots$ \\
\hline G211.47-19.27North & $18.0 \pm 3.6^{\dagger}$ & $19.0 \pm 0.4$ & $\geq 53.0^{\ddagger}$ & $\geq 17.0^{\ddagger}$ & $\leq 31.0$ & $\geq 86.0^{\ddagger}$ & $0 . \overline{10} \pm 0.02$ & $\leq 3.1$ & $\geq 6.1$ & $\leq 2.2$ & $1.0 \pm 0.1$ & $2.5 \pm 0.2$ \\
\hline G211.47-19.27South & $\leq 11.0$ & $17.0 \pm 1.1$ & $\geq 38.0^{\ddagger}$ & $\geq 16.0^{\ddagger}$ & $\geq 38.0^{\ddagger}$ & $\geq 130.0^{\ddagger}$ & $\leq 0.07$ & $\leq 2.4$ & $\leq 4.5$ & $\leq 1.3$ & $2.1 \pm 0.1$ & $1.7 \pm 0.1$ \\
\hline G211.72-19.25North & $\leq 11.0$ & $6.4 \pm 0.7$ & $\geq 36.0^{\ddagger}$ & $\geq 7.5^{\ddagger}$ & $\leq 30.0$ & $\geq 39.0^{\ddagger}$ & $\leq 0.17$ & $\leq 4.8$ & $\geq 2.1$ & $\leq 1.6$ & $0.7 \pm 0.1$ & $3.6 \pm 0.4$ \\
\hline G211.72-19.25South1 & $\leq 10.0$ & $2.0 \pm 0.2$ & $\geq 21.0^{\ddagger}$ & $\geq 7.1^{\ddagger}$ & $\geq 17.0^{\ddagger}$ & $\geq 15.0^{\ddagger}$ & $\leq 0.50$ & $\leq 3.0$ & $\leq 1.2$ & $\leq 1.3$ & $0.7 \pm 0.1$ & $2.0 \pm 0.2$ \\
\hline G212.10-19.15North1 & $39.0 \pm 6.7$ & $11.0 \pm 1.3$ & $\geq 97.0^{\ddagger}$ & $\geq 16.0^{\ddagger}$ & $\leq 42.0$ & $\geq 68.0^{\ddagger}$ & $0.35 \pm 0.07$ & $\leq 6.1$ & $\geq 2.6$ & $\leq 1.6$ & $1.8 \pm 0.2$ & $4.0 \pm 0.3$ \\
\hline G212.10-19.15North2 & $\geq 19.0^{\ddagger}$ & $17.0 \pm 1.4$ & $\geq 29.0^{\ddagger}$ & $\geq 13.0^{\ddagger}$ & $\leq 40.0$ & $\geq 74.0^{\ddagger}$ & $\geq 0.11$ & $\leq 2.2$ & $\geq 4.2$ & $\leq 2.3$ & $1.5 \pm 0.1$ & $1.7 \pm 0.1$ \\
\hline G212.10-19.15North3 & $9.6 \pm 5.1^{\dagger}$ & $16.0 \pm 2.1$ & $53.0 \pm 27.0$ & $\overline{19.0} \pm 9.6$ & $200.0 \pm 100.0$ & $1100.0 \pm 550.0$ & $0.06 \pm 0.03$ & $2 . \overline{8} \pm 2.0$ & $0 . \overline{8} \pm 0.4$ & $0 . \overline{1} \pm 0.1$ & $1.2 \pm 0.2$ & $2.0 \pm 0.1$ \\
\hline
\end{tabular}


Table 5 (continued)

$\infty$

\begin{tabular}{|c|c|c|c|c|c|c|c|c|c|c|c|c|}
\hline SCUBA-2 core & $\begin{array}{c}N\left(\mathrm{~N}_{2} \mathrm{D}^{+}\right) \\
\left(10^{11} \mathrm{~cm}^{-2}\right) \\
(2)\end{array}$ & $\begin{array}{c}N\left(\mathrm{~N}_{2} \mathrm{H}^{+}\right) \\
\left(10^{12} \mathrm{~cm}^{-2}\right) \\
(3)\end{array}$ & $\begin{array}{c}N(\mathrm{DNC}) \\
\left(10^{11} \mathrm{~cm}^{-2}\right) \\
(4)\end{array}$ & $\begin{array}{c}N\left(\mathrm{HN}^{13} \mathrm{C}\right) \\
\left(10^{11} \mathrm{~cm}^{-2}\right) \\
(5)\end{array}$ & $\begin{array}{c}N(\mathrm{CCS}) \\
\left(10^{11} \mathrm{~cm}^{-2}\right) \\
(6)\end{array}$ & $\begin{array}{c}N\left(\mathrm{HC}_{3} \mathrm{~N}\right) \\
\left(10^{11} \mathrm{~cm}^{-2}\right) \\
(7)\end{array}$ & $\frac{N\left(\mathrm{~N}_{2} \mathrm{D}^{+}\right)}{N\left(\mathrm{~N}_{2} \mathrm{H}^{+}\right)}$ & $\frac{N(\mathrm{DNC})}{N\left(\mathrm{HN}^{13} \mathrm{C}\right)}$ & $\frac{N\left(\mathrm{~N}_{2} \mathrm{H}^{+}\right)}{N(\mathrm{CCS})}$ & $\frac{N\left(\mathrm{~N}_{2} \mathrm{H}^{+}\right)}{N\left(\mathrm{HC}_{3} \mathrm{~N}\right)}$ & (12) & $\frac{\int T(\mathrm{DNC}) d v}{\int T\left(\mathrm{HN}^{13} \mathrm{C}\right) d v}$ \\
\hline G212.10-19.15South & $25.0 \pm 2.4^{\dagger}$ & $6.4 \pm 0.7$ & $\geq 46.0^{\ddagger}$ & $\geq 13.0^{\ddagger}$ & $\leq 44.0$ & $\geq 44.0^{\ddagger}$ & $0.39 \pm 0.06$ & $\leq 3.5$ & $\geq 1.5$ & $\leq 1.5$ & $0.8 \pm 0.1$ & $2.6 \pm 0.2$ \\
\hline G212.84-19.45North & $9.8 \pm 1.2^{\dagger}$ & $9.7 \pm 1.3$ & $\geq 33.0^{\ddagger}$ & $\geq 13.0^{\ddagger}$ & $\geq 22.0^{\ddagger}$ & $\geq 110.0^{\ddagger}$ & $0.10 \pm 0.02$ & $\leq 2.5$ & $\leq 4.4$ & $\leq 0.9$ & $0.6 \pm 0.1$ & $2.0 \pm 0.2$ \\
\hline G212.84-19.45South & $\leq 13.0$ & $\geq 1.8^{\ddagger}$ & $\leq 4.7$ & $\leq 4.0$ & $\leq 34.0$ & $\geq 28.0^{\ddagger}$ & $\leq 0.72$ & $\cdots$ & $\geq 0.5$ & $\leq 0.6$ & $\cdots$ & $\cdots$ \\
\hline G215.44-16.38 & $\leq 13.0$ & $5.5 \pm 0.8$ & $\geq 44.0^{\ddagger}$ & $\geq 13.0^{\ddagger}$ & $\leq 32.0$ & $\geq 71.0^{\ddagger}$ & $\leq 0.24$ & $\leq 3.4$ & $\geq 1.7$ & $\leq 0.8$ & $0.6 \pm 0.1$ & $2.7 \pm 0.2$ \\
\hline G215.87-17.62North & $\leq 14.0$ & $2.2 \pm 0.9$ & $\leq 4.8$ & $\leq 4.8$ & $\leq 29.0$ & $\leq 33.0$ & $\leq 0.64$ & $\cdots$ & $\geq 0.8$ & $\geq 0.7$ & $0.9 \pm 0.4$ & $\cdots$ \\
\hline G215.87-17.62Middle & $10.0 \pm 1.2^{\dagger}$ & $7.2 \pm 0.8$ & $\geq 27.0^{\ddagger}$ & $\geq 9.4^{\ddagger}$ & $\geq 14.0^{\ddagger}$ & $\geq 24.0^{\ddagger}$ & $0 . \overline{14} \pm 0.02$ & $\leq 2.9$ & $\leq 5.1$ & $\leq 3.0$ & $0.7 \pm 0.1$ & $2.4 \pm 0.2$ \\
\hline G215.87-17.62South & $9.2 \pm 2.6^{\dagger}$ & $\geq 5.5^{\ddagger}$ & $\geq 33.0^{\ddagger}$ & $\geq 13.0^{\ddagger}$ & $\leq 29.0$ & $\geq 52.0^{\ddagger}$ & $\leq 0.17$ & $\leq 2.5$ & $\geq 1.9$ & $\leq 1.1$ & $\ldots$ & $1.9 \pm 0.1$ \\
\hline SCOPEG139.12-03.23 & $\leq 7.3$ & $\cdots$ & $\leq 2.6$ & $\leq 2.3$ & $\cdots$ & $\cdots$ & $\cdots$ & $\cdots$ & $\cdots$ & $\cdots$ & $\cdots$ & $\cdots$ \\
\hline SCOPEG159.21-20.13 & $56.0 \pm 3.4$ & $\cdots$ & $\geq 97.0^{\ddagger}$ & $\geq 31.0^{\ddagger}$ & $\cdots$ & $\cdots$ & $\cdots$ & $\leq 3.1$ & $\cdots$ & $\cdots$ & $\cdots$ & $2.2 \pm 0.1$ \\
\hline SCOPEG159.18-20.09 & $43.0 \pm 1.3^{\dagger}$ & $\ldots$ & $660.0 \pm 330.0$ & $48.0 \pm 24.0$ & $\ldots$ & $\ldots$ & $\cdots$ & $13.8 \pm 9.7$ & $\ldots$ & $\cdots$ & $\ldots$ & $2.5 \pm 0.1$ \\
\hline SCOPEG159.22-20.11 & $140.0 \pm 4.4$ & $\cdots$ & $670.0 \pm 340.0$ & $64.0 \pm 32.0$ & $\cdots$ & $\cdots$ & $\cdots$ & $10.5 \pm 7.5$ & $\cdots$ & $\cdots$ & $\cdots$ & $2.8 \pm 0.1$ \\
\hline SCOPEG162.46-08.67 & $\geq 6.6^{\ddagger}$ & $\ldots$ & $\geq 16.0^{\ddagger}$ & $\geq 5.8^{\ddagger}$ & $\ldots$ & $\ldots$ & $\ldots$ & $\leq 2.8$ & $\ldots$ & $\ldots$ & $\ldots$ & $1.9 \pm 0.2$ \\
\hline SCOPEG162.48-08.68 & $\geq 10.0^{\ddagger}$ & $\cdots$ & $\geq 18.0^{\ddagger}$ & $\geq 5.9^{\ddagger}$ & $\cdots$ & $\cdots$ & $\cdots$ & $\leq 3.1$ & $\cdots$ & $\cdots$ & $\cdots$ & $2.4 \pm 0.2$ \\
\hline SCOPEG171.50-14.91 & $32.0 \pm 3.3$ & $\ldots$ & $\geq 78.0^{\ddagger}$ & $\geq 27.0^{\ddagger}$ & $\ldots$ & $\ldots$ & $\ldots$ & $\leq 2.9$ & $\ldots$ & . & $\ldots$ & $2.1 \pm 0.1$ \\
\hline SCOPEG172.88+02.26 & $19.0 \pm 1.2^{\dagger}$ & $\cdots$ & $\geq 37.0^{\ddagger}$ & $\geq 19.0^{\ddagger}$ & $\cdots$ & $\cdots$ & $\cdots$ & $\leq 1.9$ & $\cdots$ & $\cdots$ & $\cdots$ & $1.4 \pm 0.1$ \\
\hline SCOPEG172.88+02.27 & $30.0 \pm 3.0^{\dagger}$ & $\cdots$ & $\geq 54.0^{\ddagger}$ & $\geq 20.0^{\ddagger}$ & $\cdots$ & $\cdots$ & $\cdots$ & $\leq 2.7$ & $\cdots$ & . & $\cdots$ & $2.1 \pm 0.1$ \\
\hline SCOPEG172.89+02.27 & $\leq 6.0$ & $\cdots$ & $\leq 1.8$ & $\leq 2.0$ & $\cdots$ & $\cdots$ & . & $\cdots$ & $\cdots$ & $\cdots$ & $\cdots$ & $\cdots$ \\
\hline SCOPEG172.89+02.27 & $29.0 \pm 1.8^{\dagger}$ & $\cdots$ & $\geq 64.0^{\ddagger}$ & $\geq 16.0^{\ddagger}$ & $\cdots$ & $\cdots$ & $\cdots$ & $\leq 4.0$ & $\cdots$ & $\cdots$ & $\cdots$ & $2.8 \pm 0.1$ \\
\hline SCOPEG173.17+02.36 & $\leq 8.0$ & $\cdots$ & $9.7 \pm 5.0$ & $\leq 2.6$ & $\cdots$ & $\cdots$ & $\cdots$ & $\geq 3.7$ & $\cdots$ & $\cdots$ & $\cdots$ & $\geq 1.2$ \\
\hline SCOPEG173.17+02.36 & $\leq 8.0$ & $\cdots$ & $18.0 \pm 9.1$ & $16.0 \pm 8.1$ & $\cdots$ & $\cdots$ & $\cdots$ & $1.1 \pm 0.8$ & $\cdots$ & $\cdots$ & $\cdots$ & $1.1 \pm 0.1$ \\
\hline SCOPEG173.18+02.35 & $\leq 7.4$ & $\cdots$ & $8.0 \pm 4.1$ & $\leq 2.6$ & $\cdots$ & $\cdots$ & $\cdots$ & $\geq 3.1$ & $\cdots$ & $\cdots$ & $\cdots$ & $\geq 1.0$ \\
\hline SCOPEG173.18+02.35 & $17 . \overline{0} \pm 8.8^{\dagger}$ & $\cdots$ & $32.0 \pm 16.0$ & $24.0 \pm 12.0$ & $\cdots$ & $\cdots$ & $\cdots$ & $1.3 \pm 0.9$ & $\cdots$ & $\cdots$ & $\cdots$ & $0.9 \pm 0.1$ \\
\hline SCOPEG173.19+02.35 & $19.0 \pm 9.8^{\dagger}$ & $\ldots$ & $37.0 \pm 19.0$ & $23.0 \pm 12.0$ & $\ldots$ & $\ldots$ & .. & $1.6 \pm 1.2$ & $\ldots$ & . & $\ldots$ & $1.3 \pm 0.1$ \\
\hline SCOPEG178.27-00.60 & $27.0 \pm 5.4$ & $\cdots$ & $\geq 25.0^{\ddagger}$ & $\geq 4.4^{\ddagger}$ & $\cdots$ & $\cdots$ & $\cdots$ & $\leq 5.7$ & $\cdots$ & $\cdots$ & $\cdots$ & $4.7 \pm 0.6$ \\
\hline SCOPEG178.28-00.60 & $\geq 12.0^{\ddagger}$ & $\cdots$ & $\geq 13.0^{\ddagger}$ & $\geq 2.7^{\ddagger}$ & $\cdots$ & $\cdots$ & $\cdots$ & $\leq 4.8$ & $\cdots$ & $\cdots$ & $\cdots$ & $3.5 \pm 0.7$ \\
\hline SCOPEG195.71-02.32 & $\geq 10.0^{\ddagger}$ & $\cdots$ & $\geq 34.0^{\ddagger}$ & $\geq 7.1^{\ddagger}$ & $\cdots$ & $\cdots$ & $\cdots$ & $\leq 4.8$ & $\cdots$ & $\cdots$ & $\cdots$ & $3.3 \pm 0.4$ \\
\hline SCOPEG195.74-02.30 & $\geq 22.0^{\ddagger}$ & $\cdots$ & $\geq 58.0^{\ddagger}$ & $\geq 10.0^{\ddagger}$ & $\cdots$ & $\cdots$ & $\cdots$ & $\leq 5.8$ & $\ldots$ & $\cdots$ & $\cdots$ & $4.1 \pm 0.3$ \\
\hline SCOPEG202.30+02.53 & $9.6 \pm 1.4^{\dagger}$ & $\cdots$ & $17.0 \pm 8.5$ & $5.6 \pm 2.9$ & $\cdots$ & $\cdots$ & $\cdots$ & $3.0 \pm 2.2$ & $\cdots$ & $\cdots$ & $\cdots$ & $2.5 \pm 0.3$ \\
\hline SCOPEG202.31+02.52 & $6.0 \pm 3.2^{\dagger}$ & $\cdots$ & $24.0 \pm 12.0$ & $\leq 2.2$ & $\cdots$ & $\cdots$ & $\cdots$ & $\geq 10.9$ & $\cdots$ & $\cdots$ & $\cdots$ & $\geq 4.9$ \\
\hline SCOPEG202.32+02.53 & $\leq 6.0$ & $\cdots$ & $\leq 2.3$ & $\leq 2.2$ & $\cdots$ & $\cdots$ & $\cdots$ & $\cdots$ & $\cdots$ & $\cdots$ & $\cdots$ & $\cdots$ \\
\hline SCOPEG $202.32+02.53$ & $17.0 \pm 8.6^{\dagger}$ & $\cdots$ & $41.0 \pm 21.0$ & $5.9 \pm 3.0$ & $\cdots$ & $\cdots$ & ... & $6.9 \pm 5.0$ & $\ldots$ & $\cdots$ & $\cdots$ & $5.4 \pm 0.5$ \\
\hline SCOPEG006.01+36.74 & $25.0 \pm 2.7$ & $\cdots$ & $\geq 59.0^{\ddagger}$ & $\geq 11.0^{\ddagger}$ & $\cdots$ & $\cdots$ & $\cdots$ & $\leq 5.4$ & $\cdots$ & $\cdots$ & $\cdots$ & $3.2 \pm 0.2$ \\
\hline SCOPEG001.37+20.95 & $43.0 \pm 4.9$ & $\cdots$ & $380.0 \pm 190.0$ & $26.0 \pm 13.0$ & $\cdots$ & $\cdots$ & $\cdots$ & $14.6 \pm 10.3$ & $\cdots$ & $\cdots$ & $\cdots$ & $3.4 \pm 0.1$ \\
\hline SCOPEG010.19+02.41 & $\leq 12.0$ & $\cdots$ & $\leq 3.5$ & $\leq 3.6$ & $\cdots$ & $\cdots$ & $\cdots$ & $\cdots$ & $\cdots$ & $\cdots$ & $\cdots$ & $\cdots$ \\
\hline SCOPEG005.91-00.95 & $\leq 15.0$ & $\cdots$ & $\leq 5.7$ & $\leq 4.3$ & $\ldots$ & $\cdots$ & $\cdots$ & $\cdots$ & $\cdots$ & $\cdots$ & $\cdots$ & $\cdots$ \\
\hline SCOPEG005.88-01.01 & $\leq 11.0$ & $\cdots$ & $7.6 \pm 3.9$ & $\leq 3.1$ & $\cdots$ & $\cdots$ & $\cdots$ & $\geq 2.5$ & $\cdots$ & $\cdots$ & $\cdots$ & $\geq 1.0$ \\
\hline SCOPEG005.92-00.99 & $\leq 12.0$ & $\cdots$ & $\leq 4.7$ & $\leq 3.3$ & $\cdots$ & $\cdots$ & $\cdots$ & $\cdots$ & $\cdots$ & $\cdots$ & $\cdots$ & $\cdots$ \\
\hline SCOPEG005.92-00.99 & $\leq 11.0$ & $\cdots$ & $\leq 4.6$ & $\leq 3.3$ & $\cdots$ & $\cdots$ & $\cdots$ & $\cdots$ & $\cdots$ & $\cdots$ & $\cdots$ & $\cdots$ \\
\hline SCOPEG005.90-01.01 & $\leq 11.0$ & $\cdots$ & $\leq 4.2$ & $\leq 3.0$ & $\ldots$ & $\ldots$ & $\ldots$ & $\cdots$ & $\ldots$ & $\ldots$ & $\cdots$ & $\ldots$ \\
\hline
\end{tabular}


Table 5 (continued)

\begin{tabular}{|c|c|c|c|c|c|c|c|c|c|c|c|c|}
\hline SCUBA-2 core & $\begin{array}{c}N\left(\mathrm{~N}_{2} \mathrm{D}^{+}\right) \\
\left(10^{11} \mathrm{~cm}^{-2}\right) \\
(2)\end{array}$ & $\begin{array}{c}N\left(\mathrm{~N}_{2} \mathrm{H}^{+}\right) \\
\left(10^{12} \mathrm{~cm}^{-2}\right) \\
(3)\end{array}$ & $\begin{array}{c}N(\mathrm{DNC}) \\
\left(10^{11} \mathrm{~cm}^{-2}\right) \\
(4)\end{array}$ & $\begin{array}{c}N\left(\mathrm{HN}^{13} \mathrm{C}\right) \\
\left(10^{11} \mathrm{~cm}^{-2}\right) \\
(5)\end{array}$ & $\begin{array}{c}N(\mathrm{CCS}) \\
\left(10^{11} \mathrm{~cm}^{-2}\right) \\
(6)\end{array}$ & $\begin{array}{c}N\left(\mathrm{HC}_{3} \mathrm{~N}\right) \\
\left(10^{11} \mathrm{~cm}^{-2}\right) \\
(7)\end{array}$ & $\begin{array}{c}\frac{N\left(\mathrm{~N}_{2} \mathrm{D}^{+}\right)}{N\left(\mathrm{~N}_{2} \mathrm{H}^{+}\right)} \\
(8) \\
(8)\end{array}$ & $\frac{N(\mathrm{DNC})}{N\left(\mathrm{HN}^{13} \mathrm{C}\right)}$ & $\frac{N\left(\mathrm{~N}_{2} \mathrm{H}^{+}\right)}{N(\mathrm{CCS})}$ & $\frac{N\left(\mathrm{~N}_{2} \mathrm{H}^{+}\right)}{N\left(\mathrm{HC}_{3} \mathrm{~N}\right)}$ & $(12)$ & $\frac{\int T(\mathrm{DNC}) d v}{\int T\left(\mathrm{HN}^{13} \mathrm{C}\right) d v}$ \\
\hline SCOPEG005.90-01.01 & $\leq 10.0$ & $\cdots$ & $\leq 4.1$ & $\leq 3.0$ & $\cdots$ & $\cdots$ & $\cdots$ & $\cdots$ & $\cdots$ & $\cdots$ & $\cdots$ & $\cdots$ \\
\hline SCOPEG005.91-01.02 & $\leq 12.0$ & $\cdots$ & $\leq 3.6$ & $\leq 3.4$ & $\cdots$ & $\cdots$ & $\cdots$ & $\cdots$ & $\cdots$ & $\cdots$ & $\cdots$ & $\cdots$ \\
\hline SCOPEG005.91-01.02 & $\leq 12.0$ & $\cdots$ & $\leq 3.6$ & $\leq 3.4$ & $\cdots$ & $\cdots$ & $\cdots$ & $\cdots$ & $\cdots$ & $\cdots$ & $\cdots$ & $\cdots$ \\
\hline SCOPEG017.38+02.26 & $\leq 11.0$ & $\cdots$ & $7.6 \pm 4.0$ & $4.4 \pm 2.3$ & $\cdots$ & $\cdots$ & $\cdots$ & $1.7 \pm 1.3$ & $\cdots$ & $\cdots$ & $\cdots$ & $1.6 \pm 0.3$ \\
\hline SCOPEG017.38+02.25 & $\leq 8.6$ & $\cdots$ & $9.6 \pm 4.9$ & $\leq 2.8$ & $\cdots$ & $\cdots$ & $\cdots$ & $\geq 3.4$ & $\cdots$ & $\cdots$ & $\cdots$ & $\geq 1.6$ \\
\hline SCOPEG017.37+02.24 & $\leq 8.2$ & $\cdots$ & $9.2 \pm 4.7$ & $8.4 \pm 4.3$ & $\cdots$ & $\cdots$ & $\cdots$ & $1.1 \pm 0.8$ & $\cdots$ & $\cdots$ & $\cdots$ & $0.9 \pm 0.1$ \\
\hline SCOPEG017.36+02.23 & $4.9 \pm 1.1^{\dagger}$ & $\ldots$ & $9.2 \pm 4.7$ & $16.0 \pm 8.1$ & $\ldots$ & $\ldots$ & $\ldots$ & $0.6 \pm 0.4$ & $\ldots$ & $\ldots$ & $\ldots$ & $0.5 \pm 0.1$ \\
\hline SCOPEG014.20-00.18 & $\leq 11.0$ & $\cdots$ & $\leq 3.2$ & $\leq 3.4$ & $\cdots$ & $\cdots$ & $\cdots$ & $\cdots$ & $\cdots$ & $\cdots$ & $\cdots$ & $\cdots$ \\
\hline SCOPEG014.20-00.18 & $\leq 11.0$ & $\cdots$ & $\leq 3.2$ & $4.9 \pm 2.6$ & $\cdots$ & $\cdots$ & $\cdots$ & $\leq 0.7$ & $\cdots$ & $\cdots$ & $\cdots$ & $\leq 0.8$ \\
\hline SCOPEG014.23-00.17 & $\leq 9.1$ & $\cdots$ & $\leq 3.3$ & $18.0 \pm 9.1$ & $\cdots$ & $\cdots$ & $\cdots$ & $\leq 0.2$ & $\cdots$ & $\cdots$ & $\cdots$ & $\leq 0.3$ \\
\hline SCOPEG014.18-00.23 & $\leq 7.7$ & $\cdots$ & $\leq 2.5$ & $3.4 \pm 1.8$ & $\cdots$ & $\cdots$ & $\cdots$ & $\leq 0.7$ & $\cdots$ & $\cdots$ & $\cdots$ & $\leq 1.0$ \\
\hline SCOPEG014.18-00.23 & $\leq 7.7$ & $\cdots$ & $9.3 \pm 4.8$ & $8.5 \pm 4.3$ & $\cdots$ & $\cdots$ & $\cdots$ & $1.1 \pm 0.8$ & $\cdots$ & $\cdots$ & $\cdots$ & $0.6 \pm 0.1$ \\
\hline SCOPEG014.71-00.15 & $\leq 18.0$ & $\cdots$ & $\leq 5.0$ & $\leq 4.8$ & $\cdots$ & $\cdots$ & $\cdots$ & $\ldots$ & $\cdots$ & $\cdots$ & $\cdots$ & $\ldots$ \\
\hline SCOPEG014.72-00.20 & $\leq 13.0$ & $\cdots$ & $2.8 \pm 1.5$ & $16.0 \pm 8.1$ & $\cdots$ & $\cdots$ & $\cdots$ & $0.2 \pm 0.1$ & $\cdots$ & $\cdots$ & $\cdots$ & $0.1 \pm 0.1$ \\
\hline SCOPEG014.69-00.22 & $\leq 12.0$ & $\cdots$ & $\leq 4.3$ & $\leq 3.6$ & $\cdots$ & $\cdots$ & $\cdots$ & $\ldots$ & $\cdots$ & $\cdots$ & $\ldots$ & $\ldots$ \\
\hline SCOPEG014.71-00.22 & $\leq 12.0$ & $\cdots$ & $\leq 4.3$ & $24.0 \pm 12.0$ & $\cdots$ & $\cdots$ & $\cdots$ & $\leq 0.2$ & $\cdots$ & $\cdots$ & $\cdots$ & $\leq 0.3$ \\
\hline SCOPEG014.11-00.57 & $\leq 11.0$ & $\cdots$ & $46.0 \pm 23.0$ & $37.0 \pm 19.0$ & $\ldots$ & $\cdots$ & $\cdots$ & $1.2 \pm 0.9$ & $\cdots$ & $\ldots$ & $\ldots$ & $1.0 \pm 0.1$ \\
\hline SCOPEG014.23-00.51 & $\leq 11.0$ & $\cdots$ & $39.0 \pm 20.0$ & $25.0 \pm 13.0$ & $\cdots$ & $\cdots$ & $\cdots$ & $1.6 \pm 1.1$ & $\cdots$ & $\cdots$ & $\cdots$ & $1.2 \pm 0.1$ \\
\hline SCOPEG016.93+00.28 & $\leq 15.0$ & $\cdots$ & $\leq 4.6$ & $\leq 4.3$ & $\cdots$ & $\cdots$ & $\cdots$ & $\ldots$ & $\cdots$ & $\cdots$ & $\cdots$ & $\cdots$ \\
\hline SCOPEG016.93+00.27 & $\leq 11.0$ & $\cdots$ & $\leq 3.9$ & $\leq 3.4$ & $\cdots$ & $\cdots$ & $\cdots$ & $\cdots$ & $\cdots$ & $\cdots$ & $\ldots$ & $\cdots$ \\
\hline SCOPEG016.93+00.25 & $\leq 11.0$ & $\cdots$ & $19.0 \pm 9.6$ & $8.4 \pm 4.3$ & $\cdots$ & $\cdots$ & $\cdots$ & $2.3 \pm 1.6$ & $\cdots$ & $\cdots$ & $\cdots$ & $1.6 \pm 0.2$ \\
\hline SCOPEG016.93+00.25 & $\leq 11.0$ & $\cdots$ & $\leq 3.7$ & $\leq 3.2$ & $\cdots$ & $\cdots$ & $\cdots$ & $\ldots$ & $\cdots$ & $\cdots$ & $\ldots$ & $\cdots$ \\
\hline SCOPEG016.93+00.24 & $\leq 11.0$ & $\cdots$ & $3.4 \pm 1.8$ & $5.8 \pm 3.0$ & $\cdots$ & $\cdots$ & $\cdots$ & $0.6 \pm 0.4$ & $\cdots$ & $\cdots$ & $\cdots$ & $1.0 \pm 0.2$ \\
\hline SCOPEG016.93+00.24 & $\leq 11.0$ & $\cdots$ & $3.6 \pm 2.0$ & $4.8 \pm 2.5$ & $\cdots$ & $\cdots$ & $\cdots$ & $0.8 \pm 0.6$ & $\cdots$ & $\cdots$ & $\ldots$ & $1.2 \pm 0.3$ \\
\hline SCOPEG016.92+00.23 & $\leq 10.0$ & $\cdots$ & $\leq 3.3$ & $\leq 3.3$ & $\cdots$ & $\cdots$ & $\cdots$ & $\cdots$ & $\cdots$ & $\cdots$ & $\cdots$ & $\cdots$ \\
\hline SCOPEG016.93+00.22 & $\leq 10.0$ & $\cdots$ & $23.0 \pm 12.0$ & $16.0 \pm 8.1$ & $\cdots$ & $\cdots$ & $\cdots$ & $1.4 \pm 1.0$ & $\cdots$ & $\cdots$ & $\ldots$ & $1.4 \pm 0.1$ \\
\hline SCOPEG016.30-00.53 & $\leq 16.0$ & $\cdots$ & $\leq 6.0$ & $6.8 \pm 3.5$ & $\cdots$ & $\cdots$ & $\cdots$ & $\leq 0.9$ & $\cdots$ & $\cdots$ & $\cdots$ & $\leq 1.4$ \\
\hline SCOPEG016.34-00.59 & $\leq 12.0$ & $\cdots$ & $\leq 4.0$ & $27.0 \pm 14.0$ & $\cdots$ & $\cdots$ & $\cdots$ & $\leq 0.1$ & $\cdots$ & $\cdots$ & $\cdots$ & $\leq 0.2$ \\
\hline SCOPEG016.38-00.61 & $\leq 12.0$ & $\cdots$ & $\leq 3.7$ & $17.0 \pm 8.6$ & $\cdots$ & $\cdots$ & $\cdots$ & $\leq 0.2$ & $\cdots$ & $\cdots$ & $\cdots$ & $\leq 0.4$ \\
\hline SCOPEG016.42-00.64 & $\leq 11.0$ & $\cdots$ & $\leq 3.5$ & $14.0 \pm 7.1$ & $\cdots$ & $\cdots$ & $\cdots$ & $\leq 0.2$ & $\cdots$ & $\cdots$ & $\ldots$ & $\leq 0.4$ \\
\hline SCOPEG016.42-00.64 & $\leq 11.0$ & $\cdots$ & $\leq 3.5$ & $3.7 \pm 1.9$ & $\cdots$ & $\cdots$ & $\cdots$ & $\leq 0.9$ & $\cdots$ & $\cdots$ & $\cdots$ & $\leq 0.4$ \\
\hline SCOPEG017.22-01.46 & $\leq 13.0$ & $\cdots$ & $\leq 4.8$ & $\leq 4.1$ & $\cdots$ & $\cdots$ & $\cdots$ & $\cdots$ & $\cdots$ & $\cdots$ & $\cdots$ & $\cdots$ \\
\hline SCOPEG017.21-01.47 & $\leq 13.0$ & $\cdots$ & $\leq 4.7$ & $\leq 4.5$ & $\cdots$ & $\cdots$ & $\cdots$ & $\cdots$ & $\cdots$ & $\cdots$ & $\cdots$ & $\cdots$ \\
\hline SCOPEG023.63+00.59 & $\leq 11.0$ & $\cdots$ & $\leq 3.9$ & $\leq 3.3$ & $\cdots$ & $\cdots$ & $\cdots$ & $\cdots$ & $\cdots$ & $\cdots$ & $\cdots$ & $\cdots$ \\
\hline SCOPEG023.69+00.59 & $\leq 12.0$ & $\cdots$ & $\leq 3.6$ & $22.0 \pm 11.0$ & $\cdots$ & $\cdots$ & $\cdots$ & $\leq 0.2$ & $\cdots$ & $\cdots$ & $\cdots$ & $\leq 0.3$ \\
\hline SCOPEG024.02+00.24 & $\leq 11.0$ & $\cdots$ & $\leq 3.7$ & $\leq 3.5$ & $\cdots$ & $\cdots$ & $\cdots$ & $\cdots$ & $\cdots$ & $\cdots$ & $\cdots$ & $\cdots$ \\
\hline SCOPEG024.02+00.21 & $\leq 9.6$ & $\cdots$ & $\leq 3.0$ & $\leq 2.9$ & $\cdots$ & $\cdots$ & $\cdots$ & $\cdots$ & $\cdots$ & $\cdots$ & $\cdots$ & $\cdots$ \\
\hline SCOPEG023.32-00.29 & $\leq 11.0$ & $\cdots$ & $\leq 3.5$ & $18.0 \pm 9.1$ & $\cdots$ & $\cdots$ & $\cdots$ & $\leq 0.2$ & $\cdots$ & $\cdots$ & $\cdots$ & $\leq 0.3$ \\
\hline SCOPEG026.50+00.71 & $\leq 12.0$ & $\cdots$ & $\leq 4.3$ & $17.0 \pm 8.6$ & $\ldots$ & $\cdots$ & $\ldots$ & $\leq 0.3$ & $\cdots$ & $\cdots$ & $\cdots$ & $\leq 0.4$ \\
\hline
\end{tabular}


Table 5 (continued)

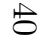

\begin{tabular}{|c|c|c|c|c|c|c|c|c|c|c|c|c|}
\hline SCUBA-2 core & $\begin{array}{c}N\left(\mathrm{~N}_{2} \mathrm{D}^{+}\right) \\
\left(10^{11} \mathrm{~cm}^{-2}\right) \\
(2)\end{array}$ & $\begin{array}{c}N\left(\mathrm{~N}_{2} \mathrm{H}^{+}\right) \\
\left(10^{12} \mathrm{~cm}^{-2}\right) \\
(3)\end{array}$ & $\begin{array}{c}N(\mathrm{DNC}) \\
\left(10^{11} \mathrm{~cm}^{-2}\right) \\
(4)\end{array}$ & $\begin{array}{c}N\left(\mathrm{HN}^{13} \mathrm{C}\right) \\
\left(10^{11} \mathrm{~cm}^{-2}\right) \\
(5)\end{array}$ & $\begin{array}{c}N(\mathrm{CCS}) \\
\left(10^{11} \mathrm{~cm}^{-2}\right) \\
(6)\end{array}$ & $\begin{array}{c}N\left(\mathrm{HC}_{3} \mathrm{~N}\right) \\
\left(10^{11} \mathrm{~cm}^{-2}\right) \\
(7)\end{array}$ & $\frac{N\left(\mathrm{~N}_{2} \mathrm{D}^{+}\right)}{N\left(\mathrm{~N}_{2} \mathrm{H}^{+}\right)}$ & $\frac{N(\mathrm{DNC})}{N\left(\mathrm{HN}^{13} \mathrm{C}\right)}$ & $\frac{N\left(\mathrm{~N}_{2} \mathrm{H}^{+}\right)}{N(\mathrm{CCS})}$ & $\frac{N\left(\mathrm{~N}_{2} \mathrm{H}^{+}\right)}{N\left(\mathrm{HC}_{3} \mathrm{~N}\right)}$ & (12) & $\frac{\int T(\mathrm{DNC}) d v}{\int T\left(\mathrm{HN}^{13} \mathrm{C}\right) d v}$ \\
\hline SCOPEG037.92+02.14 & $\leq 6.2$ & $\cdots$ & $\leq 2.2$ & $2.8 \pm 1.5$ & $\cdots$ & $\cdots$ & $\cdots$ & $\leq 0.8$ & $\cdots$ & $\cdots$ & $\cdots$ & $\leq 1.2$ \\
\hline SCOPEG033.74-00.01 & $\leq 7.3$ & $\cdots$ & $13.0 \pm 6.6$ & $36.0 \pm 18.0$ & $\cdots$ & $\cdots$ & $\cdots$ & $0.4 \pm 0.3$ & $\cdots$ & $\cdots$ & $\cdots$ & $0.3 \pm 0.1$ \\
\hline SCOPEG039.74+01.99 & $\leq 6.5$ & $\cdots$ & $15.0 \pm 7.6$ & $7.0 \pm 3.5$ & $\cdots$ & $\cdots$ & $\cdots$ & $2.1 \pm 1.5$ & $\cdots$ & $\cdots$ & $\cdots$ & $1.7 \pm 0.2$ \\
\hline SCOPEG035.48-00.29 & $\leq 9.3$ & $\cdots$ & $9.6 \pm 4.9$ & $\leq 2.9$ & $\cdots$ & $\cdots$ & $\cdots$ & $\geq 3.3$ & $\cdots$ & $\cdots$ & $\cdots$ & $\geq 1.6$ \\
\hline SCOPEG035.48-00.29 & $13.0 \pm 1.1^{\dagger}$ & $\cdots$ & $36.0 \pm 18.0$ & $20.0 \pm 10.0$ & $\cdots$ & $\cdots$ & $\cdots$ & $1.8 \pm 1.3$ & $\cdots$ & $\cdots$ & $\cdots$ & $1.4 \pm 0.1$ \\
\hline SCOPEG035.52-00.27 & $\leq 7.7$ & $\cdots$ & $46.0 \pm 23.0$ & $29.0 \pm 15.0$ & $\cdots$ & $\cdots$ & $\cdots$ & $1.6 \pm 1.1$ & $\cdots$ & $\cdots$ & $\cdots$ & $1.2 \pm 0.1$ \\
\hline SCOPEG035.48-00.31 & $23.0 \pm 12.0^{\dagger}$ & $\cdots$ & $39.0 \pm 20.0$ & $25.0 \pm 13.0$ & $\cdots$ & $\cdots$ & $\cdots$ & $1.6 \pm 1.1$ & $\cdots$ & $\cdots$ & $\cdots$ & $1.3 \pm 0.1$ \\
\hline SCOPEG034.75-01.38 & $\leq 8.2$ & $\cdots$ & $29.0 \pm 15.0$ & $34.0 \pm 17.0$ & $\cdots$ & $\cdots$ & $\cdots$ & $0.9 \pm 0.6$ & $\cdots$ & $\cdots$ & $\cdots$ & $0.7 \pm 0.1$ \\
\hline SCOPEG035.36-01.77 & $\leq 8.1$ & $\cdots$ & $\leq 2.9$ & $3.1 \pm 1.6$ & $\cdots$ & $\cdots$ & $\cdots$ & $\leq 0.9$ & $\cdots$ & $\cdots$ & $\cdots$ & $\leq 1.3$ \\
\hline SCOPEG035.36-01.78 & $\leq 6.4$ & $\cdots$ & $13.0 \pm 6.5$ & $\leq 2.0$ & $\cdots$ & $\cdots$ & $\cdots$ & $\geq 6.5$ & $\cdots$ & $\cdots$ & $\cdots$ & $\geq 2.5$ \\
\hline SCOPEG035.35-01.80 & $\leq 6.2$ & $\cdots$ & $8.7 \pm 4.4$ & $\leq 1.9$ & $\cdots$ & $\cdots$ & $\cdots$ & $\geq 4.6$ & $\cdots$ & $\cdots$ & $\cdots$ & $\geq 1.7$ \\
\hline SCOPEG057.11+03.66 & $8.1 \pm 0.7^{\dagger}$ & $\cdots$ & $\geq 24.0^{\ddagger}$ & $\geq 10.0^{\ddagger}$ & $\cdots$ & $\cdots$ & $\cdots$ & $\leq 2.4$ & $\cdots$ & $\cdots$ & $\cdots$ & $2.0 \pm 0.2$ \\
\hline SCOPEG057.10+03.63 & $6.2 \pm 1.0^{\dagger}$ & $\cdots$ & $\geq 29.0^{\ddagger}$ & $\geq 13.0^{\ddagger}$ & $\cdots$ & $\cdots$ & $\cdots$ & $\leq 2.2$ & $\cdots$ & $\cdots$ & $\cdots$ & $1.7 \pm 0.1$ \\
\hline SCOPEG069.80-01.67 & $\leq 7.2$ & $\cdots$ & $24.0 \pm 12.0$ & $20.0 \pm 10.0$ & $\cdots$ & $\cdots$ & $\cdots$ & $1.2 \pm 0.8$ & $\cdots$ & $\cdots$ & $\cdots$ & $0.9 \pm 0.1$ \\
\hline SCOPEG069.81-01.67 & $5.4 \pm 2.9^{\dagger}$ & $\cdots$ & $23.0 \pm 12.0$ & $19.0 \pm 9.5$ & $\cdots$ & $\cdots$ & $\ldots$ & $1.2 \pm 0.9$ & $\cdots$ & $\cdots$ & $\ldots$ & $1.0 \pm 0.1$ \\
\hline SCOPEG070.40-01.39 & $\leq 6.2$ & $\cdots$ & $\geq 8.2^{\ddagger}$ & $\geq 8.1^{\ddagger}$ & $\cdots$ & $\cdots$ & $\cdots$ & $\leq 1.0$ & $\cdots$ & $\cdots$ & $\cdots$ & $0.8 \pm 0.1$ \\
\hline SCOPEG074.10+00.11 & $7.6 \pm 2.5^{\dagger}$ & $\cdots$ & $\geq 19.0^{\ddagger}$ & $\geq 13.0^{\ddagger}$ & $\cdots$ & $\cdots$ & $\cdots$ & $\leq 1.5$ & $\cdots$ & $\cdots$ & $\ldots$ & $0.9 \pm 0.1$ \\
\hline SCOPEG074.11+00.11 & $14.0 \pm 2.8^{\dagger}$ & $\cdots$ & $\geq 34.0^{\ddagger}$ & $\geq 17.0^{\ddagger}$ & $\cdots$ & $\cdots$ & $\cdots$ & $\leq 2.0$ & $\cdots$ & $\cdots$ & $\cdots$ & $1.6 \pm 0.1$ \\
\hline SCOPEG082.36-01.83 & $\geq 10.0^{\ddagger}$ & $\cdots$ & $\geq 26.0^{\ddagger}$ & $\geq 11.0^{\ddagger}$ & $\cdots$ & $\cdots$ & $\cdots$ & $\leq 2.4$ & $\cdots$ & $\cdots$ & $\cdots$ & $1.7 \pm 0.1$ \\
\hline SCOPEG082.40-01.84 & $\geq 14.0^{\ddagger}$ & $\cdots$ & $\geq 32.0^{\ddagger}$ & $\geq 15.0^{\ddagger}$ & $\cdots$ & $\cdots$ & $\cdots$ & $\leq 2.1$ & $\cdots$ & $\cdots$ & $\cdots$ & $1.6 \pm 0.1$ \\
\hline SCOPEG082.41-01.84 & $17.0 \pm 1.0^{\dagger}$ & $\cdots$ & $\geq 45.0^{\ddagger}$ & $\geq 21.0^{\ddagger}$ & $\cdots$ & $\cdots$ & $\cdots$ & $\leq 2.1$ & $\cdots$ & $\cdots$ & $\ldots$ & $2.2 \pm 0.1$ \\
\hline SCOPEG082.42-01.84 & $21.0 \pm 1.5$ & $\cdots$ & $\geq 38.0^{\ddagger}$ & $\geq 18.0^{\ddagger}$ & $\cdots$ & $\cdots$ & $\cdots$ & $\leq 2.1$ & $\cdots$ & $\cdots$ & $\cdots$ & $1.7 \pm 0.1$ \\
\hline SCOPEG091.86+04.17 & $\leq 8.0$ & $\cdots$ & $\geq 15.0^{\ddagger}$ & $\geq 8.4^{\ddagger}$ & $\cdots$ & $\ldots$ & $\ldots$ & $\leq 1.8$ & $\cdots$ & $\cdots$ & $\ldots$ & $1.5 \pm 0.2$ \\
\hline SCOPEG091.86+04.17 & $\leq 8.2$ & $\cdots$ & $\leq 2.9$ & $\leq 3.0$ & $\cdots$ & $\cdots$ & $\cdots$ & $\cdots$ & $\cdots$ & $\cdots$ & $\cdots$ & $\cdots$ \\
\hline SCOPEG091.85+04.12 & $12.0 \pm 3.3$ & $\cdots$ & $\geq 39.0^{\ddagger}$ & $\geq 16.0^{\ddagger}$ & $\cdots$ & $\cdots$ & $\cdots$ & $\leq 2.4$ & $\cdots$ & $\cdots$ & $\ldots$ & $1.9 \pm 0.1$ \\
\hline SCOPEG092.03+03.93 & $\leq 5.7$ & $\cdots$ & $\leq 2.1$ & $\leq 2.4$ & $\cdots$ & $\cdots$ & $\cdots$ & $\cdots$ & $\cdots$ & $\cdots$ & $\cdots$ & $\cdots$ \\
\hline SCOPEG092.27+03.79 & $18.0 \pm 1.5$ & $\cdots$ & $\geq 30.0^{\ddagger}$ & $\geq 21.0^{\ddagger}$ & $\cdots$ & $\cdots$ & $\cdots$ & $\leq 1.4$ & $\cdots$ & $\cdots$ & $\ldots$ & $1.1 \pm 0.1$ \\
\hline SCOPEG087.06-04.19 & $3.7 \pm 0.6^{\dagger}$ & $\cdots$ & $15.0 \pm 7.5$ & $5.9 \pm 3.0$ & $\cdots$ & $\cdots$ & $\cdots$ & $2.5 \pm 1.8$ & $\cdots$ & $\cdots$ & $\cdots$ & $2.0 \pm 0.2$ \\
\hline SCOPEG089.64-06.62 & $\geq 5.4^{\ddagger}$ & $\cdots$ & $\geq 21.0^{\ddagger}$ & $\geq 7.6^{\ddagger}$ & $\cdots$ & $\cdots$ & $\cdots$ & $\leq 2.8$ & $\cdots$ & $\cdots$ & $\ldots$ & $2.3 \pm 0.2$ \\
\hline SCOPEG105.37+09.84 & $\leq 6.9$ & $\cdots$ & $15.0 \pm 7.6$ & $8.3 \pm 4.2$ & $\cdots$ & $\cdots$ & $\cdots$ & $1.8 \pm 1.3$ & $\cdots$ & $\cdots$ & $\cdots$ & $1.7 \pm 0.2$ \\
\hline SCOPEG105.41+09.88 & $\leq 6.7$ & $\cdots$ & $\leq 2.2$ & $\leq 2.2$ & $\cdots$ & $\cdots$ & $\cdots$ & $\ldots$ & $\cdots$ & $\cdots$ & $\cdots$ & $\ldots$ \\
\hline SCOPEG093.53-04.26 & $17.0 \pm 1.2^{\dagger}$ & $\cdots$ & $\geq 42.0^{\ddagger}$ & $\geq 23.0^{\ddagger}$ & $\cdots$ & $\cdots$ & $\cdots$ & $\leq 1.8$ & $\cdots$ & $\cdots$ & $\cdots$ & $1.4 \pm 0.1$ \\
\hline SCOPEG093.54-04.28 & $15.0 \pm 1.2^{\dagger}$ & $\cdots$ & $\geq 41.0^{\ddagger}$ & $\geq 16.0^{\ddagger}$ & $\ldots$ & $\ldots$ & . & $\leq 2.6$ & $\ldots$ & . & $\ldots$ & $1.9 \pm 0.1$ \\
\hline SCOPEG107.16+05.45 & $14.0 \pm 7.1^{\dagger}$ & $\cdots$ & $52.0 \pm 26.0$ & $9.4 \pm 4.7$ & $\cdots$ & $\cdots$ & $\cdots$ & $5.5 \pm 3.9$ & .. & $\cdots$ & $\cdots$ & $3.7 \pm 0.3$ \\
\hline SCOPEG107.30+05.64 & $\leq 6.2$ & $\cdots$ & $\geq 22.0^{\ddagger}$ & $\geq 16.0^{\ddagger}$ & $\cdots$ & $\ldots$ & . & $\leq 1.4$ & $\ldots$ & $\ldots$ & $\ldots$ & $1.3 \pm 0.1$ \\
\hline SCOPEG107.18+05.43 & $7.4 \pm 3.9^{\dagger}$ & $\cdots$ & $18.0 \pm 9.0$ & $10.0 \pm 5.0$ & $\cdots$ & $\cdots$ & $\cdots$ & $1.8 \pm 1.3$ & $\cdots$ & $\cdots$ & $\ldots$ & $1.5 \pm 0.1$ \\
\hline SCOPEG109.81+02.70 & $\leq 6.7$ & $\ldots$ & $\geq 21.0^{\ddagger}$ & $\geq 7.4^{\ddagger}$ & $\ldots$ & $\ldots$ & $\ldots$ & $\leq 2.8$ & $\ldots$ & $\ldots$ & $\ldots$ & $2.1 \pm 0.2$ \\
\hline
\end{tabular}

Noте-All values are listed for the source whose peak temperature is higher than 3 $\sigma$. Column 1: SCUBA-2 core name, Column 2-7: Column

densities of $\mathrm{N}_{2} \mathrm{D}^{+}, \mathrm{N}_{2} \mathrm{H}^{+}$, DNC, $\mathrm{HN}^{13} \mathrm{C}, 82 \mathrm{GHz}$ CCS, and $\mathrm{HC}_{3} \mathrm{~N}$, Column 8-11: Ratio of the column densities, Column 12: Mach number of $\mathrm{N}_{2} \mathrm{H}^{+}$line, and Column 13: Integrated intensity ratio of DNC to $\mathrm{HN}^{13} \mathrm{C}$ lines. ' $\dagger$ ' indicates that the column density is derived from dust temperature and ' $\ddagger$ ' indicates the lower limit when the assumed $T_{\text {ex }}$ is too low to constrain the upper bound. 
Table 7. Minimum, maximum, mean, and median of column-density ratios of $\mathrm{N}_{2} \mathrm{D}^{+} / \mathrm{N}_{2} \mathrm{H}^{+}$, DNC/ $\mathrm{HN}^{13} \mathrm{C}, \mathrm{N}_{2} \mathrm{H}^{+} / \mathrm{CCS}$, and $\mathrm{N}_{2} \mathrm{H}^{+} / \mathrm{HC}_{3} \mathrm{~N}$ for $207 \mathrm{SCUBA}-2$ cores in different environments

\begin{tabular}{|c|c|c|c|c|c|c|c|c|c|c|c|c|c|c|c|c|c|}
\hline \multirow[t]{2}{*}{ Environment } & \multirow[t]{2}{*}{ Source } & \multicolumn{4}{|c|}{$\frac{\mathrm{N}\left(\mathrm{N}_{2} \mathrm{D}^{+}\right)}{\mathrm{N}\left(\mathrm{N}_{2} \mathrm{H}^{+}\right)}$} & \multicolumn{4}{|c|}{$\frac{\mathrm{N}(\mathrm{DNC})}{\mathrm{N}\left(\mathrm{HN}^{13} \mathrm{C}\right)}$} & \multicolumn{4}{|c|}{$\frac{\mathrm{N}\left(\mathrm{N}_{2} \mathrm{H}^{+}\right)}{\mathrm{N}(\mathrm{CCS})}$} & \multicolumn{4}{|c|}{$\frac{\mathrm{N}\left(\mathrm{N}_{2} \mathrm{H}^{+}\right)}{\mathrm{N}\left(\mathrm{HC}_{3} \mathrm{~N}\right)}$} \\
\hline & & $\min$. & $\max$. & mean & median & $\min$. & $\max$ & mean & median & $\min$. & $\max$ & mean & median & $\min$. & $\max$. & mean & median \\
\hline \multirow{3}{*}{ Orion region } & Both & 0.04 & 0.4 & 0.2 & 0.2 & 0.7 & 7.7 & 3.3 & 3.0 & 0.8 & 20.8 & 8.7 & 7.0 & 0.1 & 11.2 & 2.7 & 2.1 \\
\hline & Starless core & 0.06 & 0.3 & 0.2 & 0.2 & 0.7 & 7.7 & 4.0 & 4.0 & 7.6 & 7.6 & 7.6 & 7.6 & 0.6 & 8.2 & 3.2 & 2.4 \\
\hline & Protostellar core & 0.04 & 0.4 & 0.2 & 0.1 & 1.0 & 5.7 & 2.9 & 2.7 & 0.8 & 20.8 & 8.9 & 5.3 & 0.1 & 11.2 & 2.4 & 1.5 \\
\hline \multirow{3}{*}{$\lambda$ Orionis } & Both & 0.08 & 0.2 & 0.1 & 0.2 & 1.8 & 5.7 & 4.0 & 3.8 & 1.2 & 1.2 & 1.2 & 1.2 & 0.5 & 2.6 & 1.3 & 1.4 \\
\hline & Starless core & $\ldots$ & $\ldots$ & $\ldots$ & $\cdots$ & $\ldots$ & $\cdots$ & $\ldots$ & $\ldots$ & $\cdots$ & $\ldots$ & $\ldots$ & $\ldots$ & $\ldots$ & $\ldots$ & $\cdots$ & $\cdots$ \\
\hline & Protostellar core & 0.08 & 0.2 & 0.1 & 0.2 & 1.8 & 5.7 & 4.0 & 3.8 & 1.2 & 1.2 & 1.2 & 1.2 & 0.5 & 2.6 & 1.3 & 1.4 \\
\hline \multirow{3}{*}{ Orion A } & Both & 0.05 & 0.4 & 0.2 & 0.1 & 0.7 & 7.7 & 3.4 & 2.9 & 0.8 & 20.8 & 9.7 & 7.3 & 0.1 & 11.2 & 2.9 & 2.2 \\
\hline & Starless core & 0.06 & 0.3 & 0.2 & 0.2 & 0.7 & 7.7 & 4.0 & 4.1 & 7.6 & 7.6 & 7.6 & 7.6 & 1.1 & 4.7 & 2.8 & 2.2 \\
\hline & Protostellar core & 0.05 & 0.4 & 0.2 & 0.1 & 1.8 & 4.0 & 2.8 & 2.7 & 0.8 & 20.8 & 10.0 & 7.0 & 0.1 & 11.2 & 2.9 & 2.4 \\
\hline \multirow{3}{*}{ Orion B } & Both & 0.04 & 0.3 & 0.2 & 0.2 & 1.0 & 5.1 & 2.6 & 2.7 & $\ldots$ & $\ldots$ & $\ldots$ & $\ldots$ & 0.6 & 8.2 & 3.3 & 1.9 \\
\hline & Starless core & 0.15 & 0.2 & 0.2 & 0.2 & 3.0 & 5.1 & 3.8 & 3.2 & $\ldots$ & $\ldots$ & $\ldots$ & $\ldots$ & 0.6 & 8.2 & 4.3 & 4.3 \\
\hline & Protostellar core & 0.04 & 0.3 & 0.2 & 0.2 & 1.0 & 2.7 & 1.7 & 1.6 & $\cdots$ & $\ldots$ & $\ldots$ & $\ldots$ & 1.2 & 1.3 & 1.3 & 1.3 \\
\hline \multirow{3}{*}{ Galactic plane } & Both & $\cdots$ & $\cdots$ & $\cdots$ & $\ldots$ & 0.2 & 2.3 & 1.2 & 1.2 & $\cdots$ & $\cdots$ & $\ldots$ & $\ldots$ & $\cdots$ & $\ldots$ & $\cdots$ & $\ldots$ \\
\hline & Starless core & $\ldots$ & $\ldots$ & $\ldots$ & $\ldots$ & 0.6 & 2.3 & 1.2 & 0.8 & $\ldots$ & $\ldots$ & $\ldots$ & $\ldots$ & $\ldots$ & $\ldots$ & $\ldots$ & $\ldots$ \\
\hline & Protostellar core & $\ldots$ & $\ldots$ & $\ldots$ & $\ldots$ & 0.2 & 2.1 & 1.2 & 1.2 & $\ldots$ & $\ldots$ & $\ldots$ & $\ldots$ & $\ldots$ & $\ldots$ & $\ldots$ & $\ldots$ \\
\hline \multirow{3}{*}{ High latitudes } & Both & $\ldots$ & $\ldots$ & $\ldots$ & $\ldots$ & 0.6 & 14.6 & 4.5 & 1.8 & $\ldots$ & $\ldots$ & $\ldots$ & $\ldots$ & $\ldots$ & $\ldots$ & $\ldots$ & $\ldots$ \\
\hline & Starless core & $\ldots$ & $\ldots$ & $\ldots$ & $\ldots$ & 6.9 & 14.6 & 10.8 & 10.8 & $\ldots$ & $\ldots$ & $\ldots$ & $\ldots$ & $\ldots$ & $\ldots$ & $\ldots$ & $\ldots$ \\
\hline & Protostellar core & $\ldots$ & $\ldots$ & $\ldots$ & $\ldots$ & 0.6 & 13.8 & 3.6 & 1.8 & $\ldots$ & $\ldots$ & $\ldots$ & $\ldots$ & $\ldots$ & $\ldots$ & $\ldots$ & $\ldots$ \\
\hline \multirow{3}{*}{$\begin{array}{l}\text { High latitudes } \\
\quad(\lesssim 1.1 \mathrm{kpc})\end{array}$} & Both & $\ldots$ & $\ldots$ & $\cdots$ & $\cdots$ & 1.8 & 14.6 & 7.3 & 6.2 & $\ldots$ & $\ldots$ & $\ldots$ & $\ldots$ & 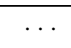 & $\cdots$ & $\cdots$ & $\cdots$ \\
\hline & Starless core & $\cdots$ & $\ldots$ & $\ldots$ & $\ldots$ & 6.9 & 14.6 & 10.8 & 10.8 & $\cdots$ & $\cdots$ & $\ldots$ & $\cdots$ & $\cdots$ & $\cdots$ & $\cdots$ & $\cdots$ \\
\hline & Protostellar core & $\ldots$ & $\ldots$ & $\ldots$ & $\ldots$ & 1.8 & 13.8 & 6.2 & 4.3 & $\ldots$ & $\ldots$ & $\ldots$ & $\ldots$ & $\ldots$ & $\ldots$ & $\ldots$ & $\ldots$ \\
\hline
\end{tabular}

Note-Only cores where both column densities are successfully estimated are considered. The $\mathrm{N}_{2} \mathrm{H}^{+}$, CCS, and $\mathrm{HC}_{3} \mathrm{~N}$ data are available for only

the Orion region. 

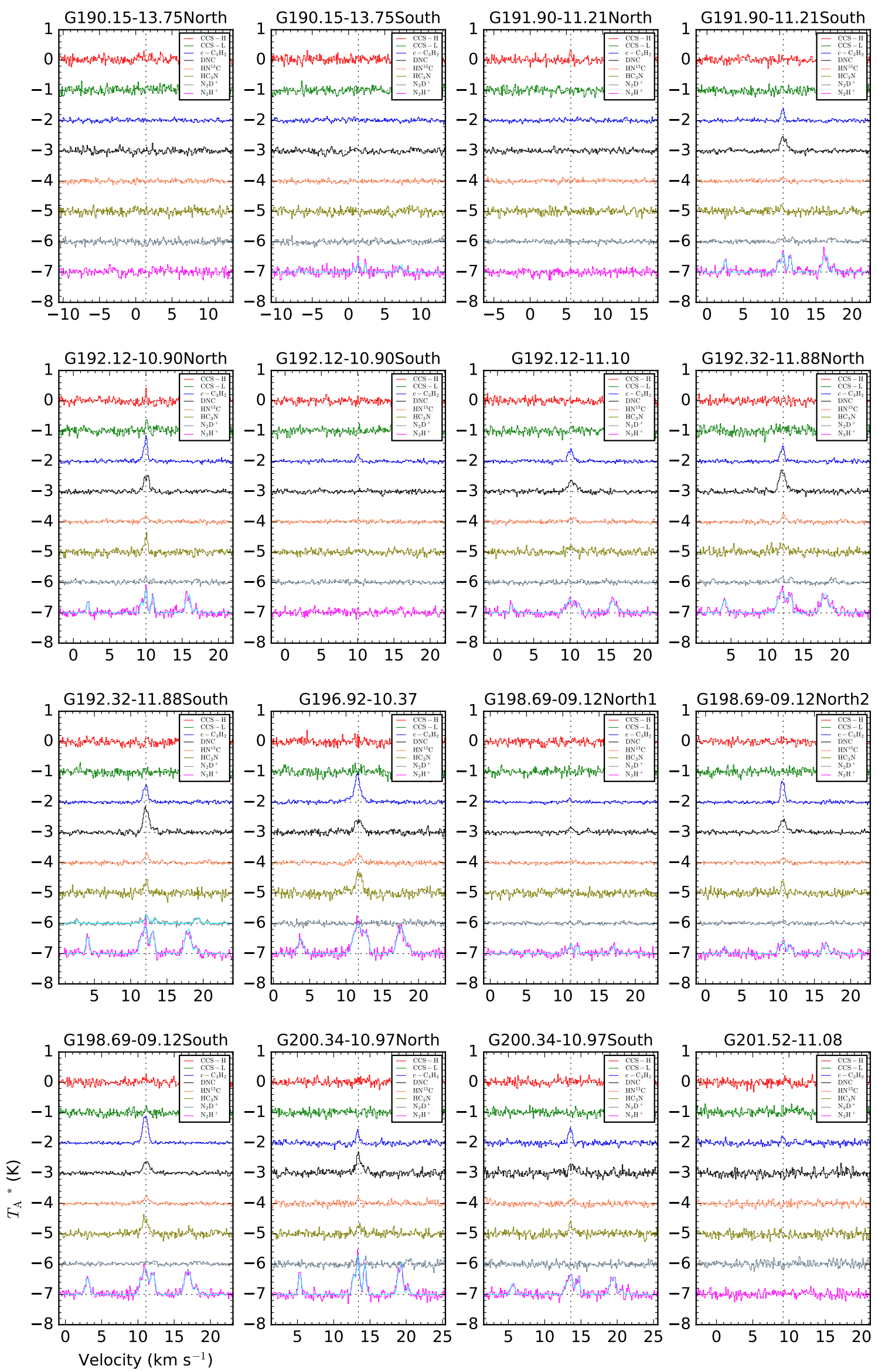

Figure 3. Spectra of 207 SCUBA-2 cores in Planck Galactic Cold Clump cores in eight molecular lines. A vertical line indicates a systemic velocity. A horizontal line represents $0 \mathrm{~K}$ in $T_{\mathrm{A}}^{*}$ scale. For the $\mathbf{N}_{2} \mathbf{H}^{+}$and $\mathbf{N}_{2} \mathbf{D}^{+}$emission, the cyan line represents the hyperfine structure fitting to result. 

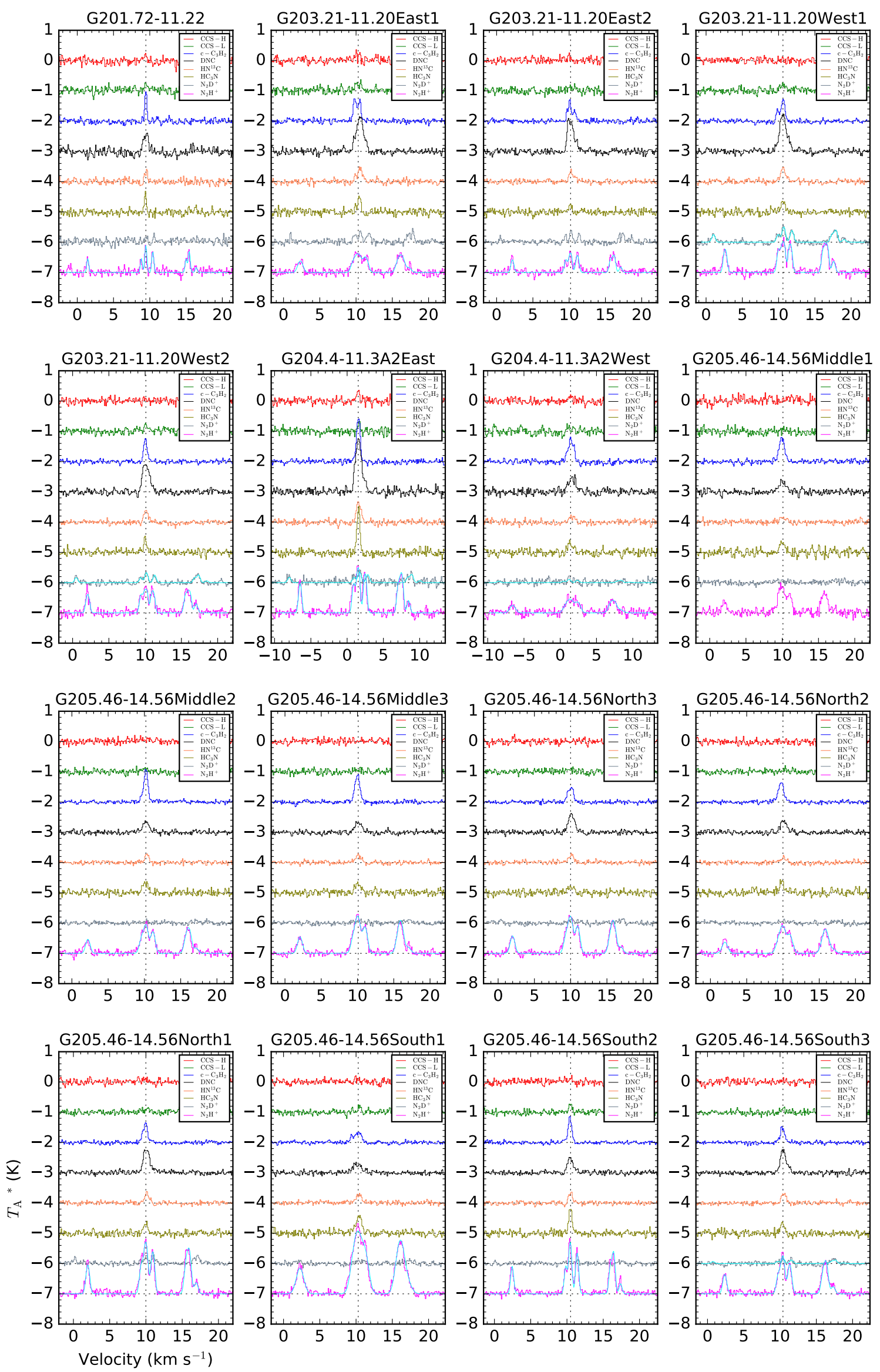

Figure 3. (continued) 

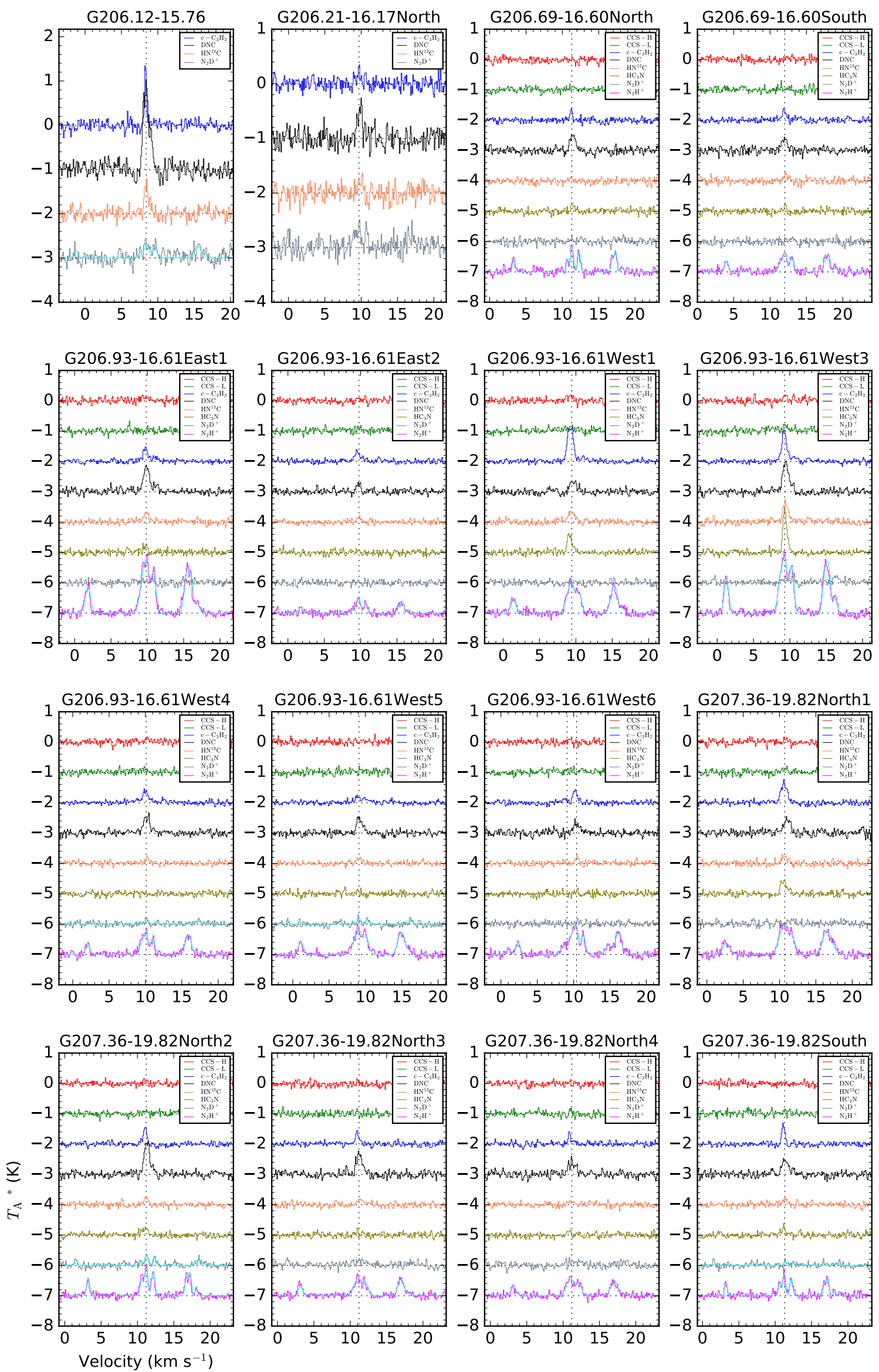

Figure 3. (continued) 

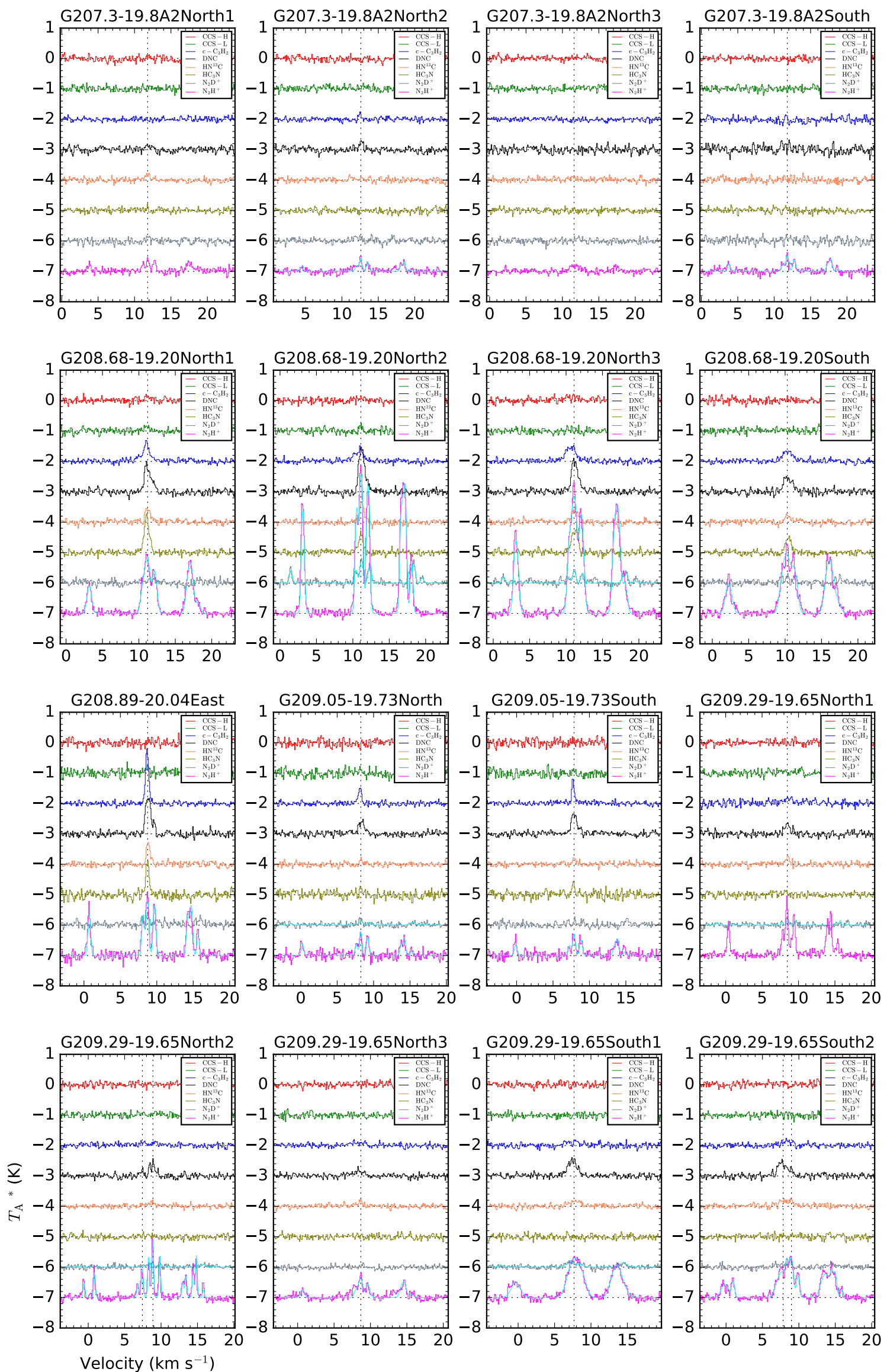

Figure 3. (continued) 

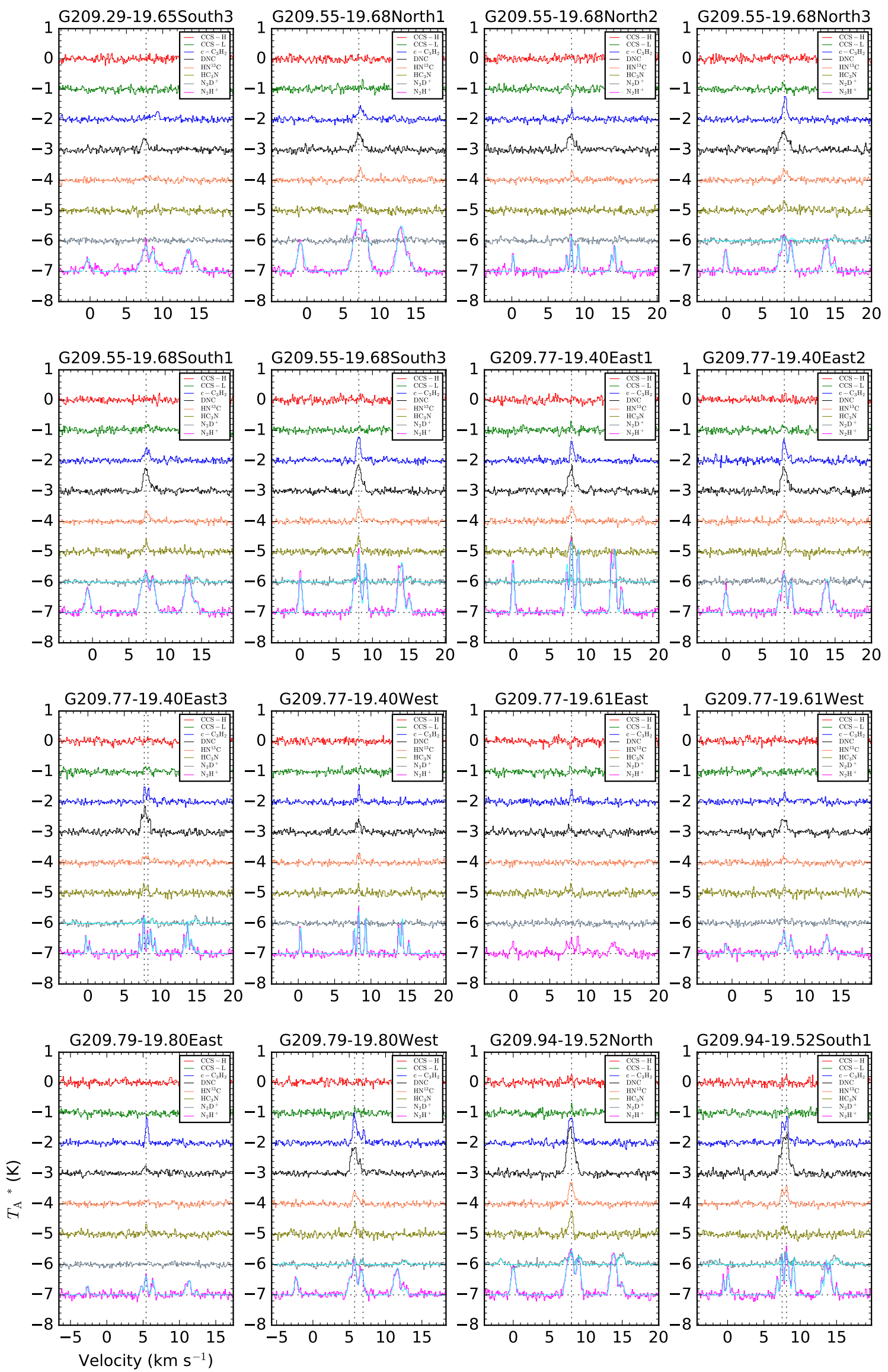

Figure 3. (continued) 

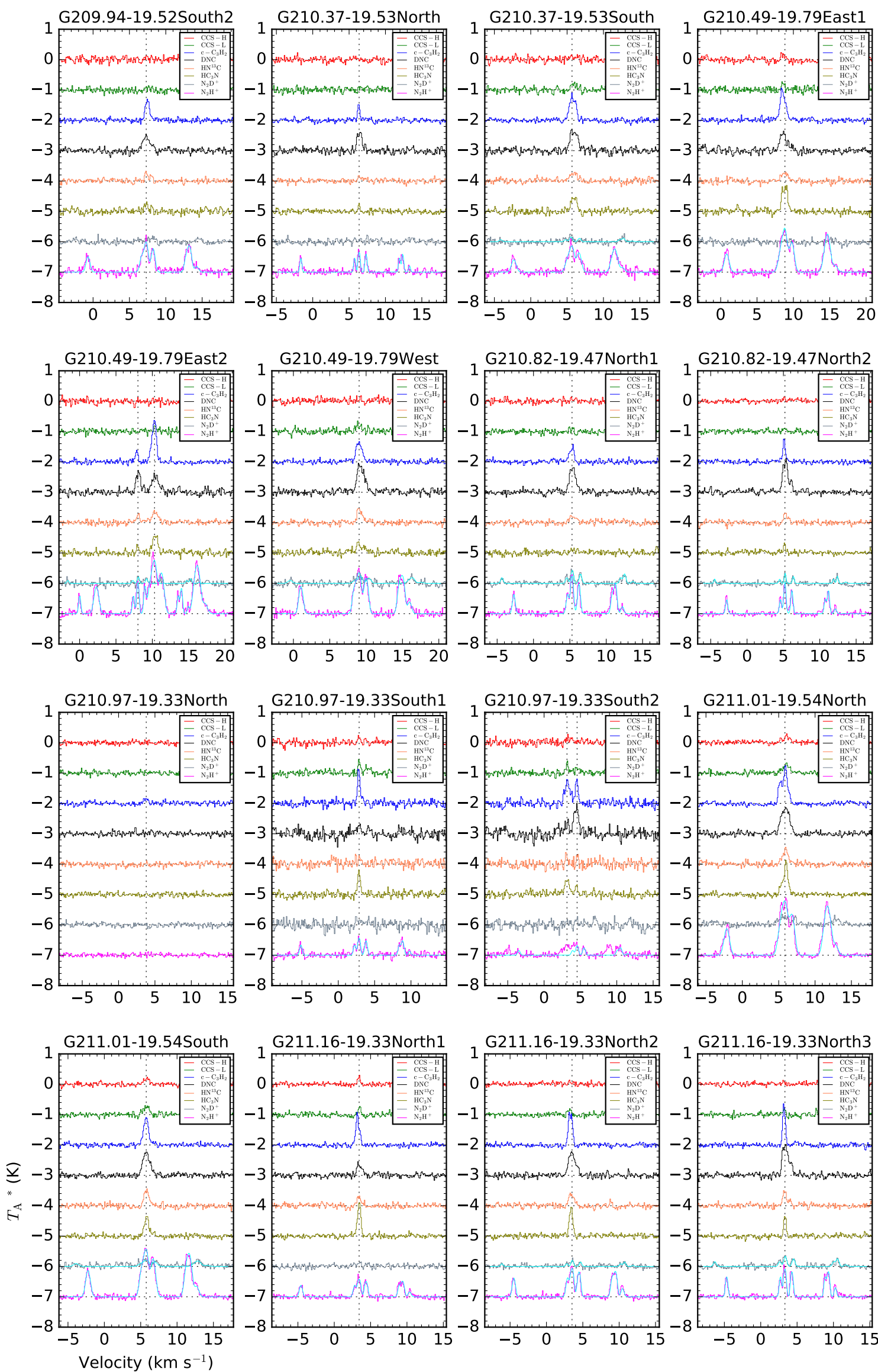

Figure 3. (continued) 

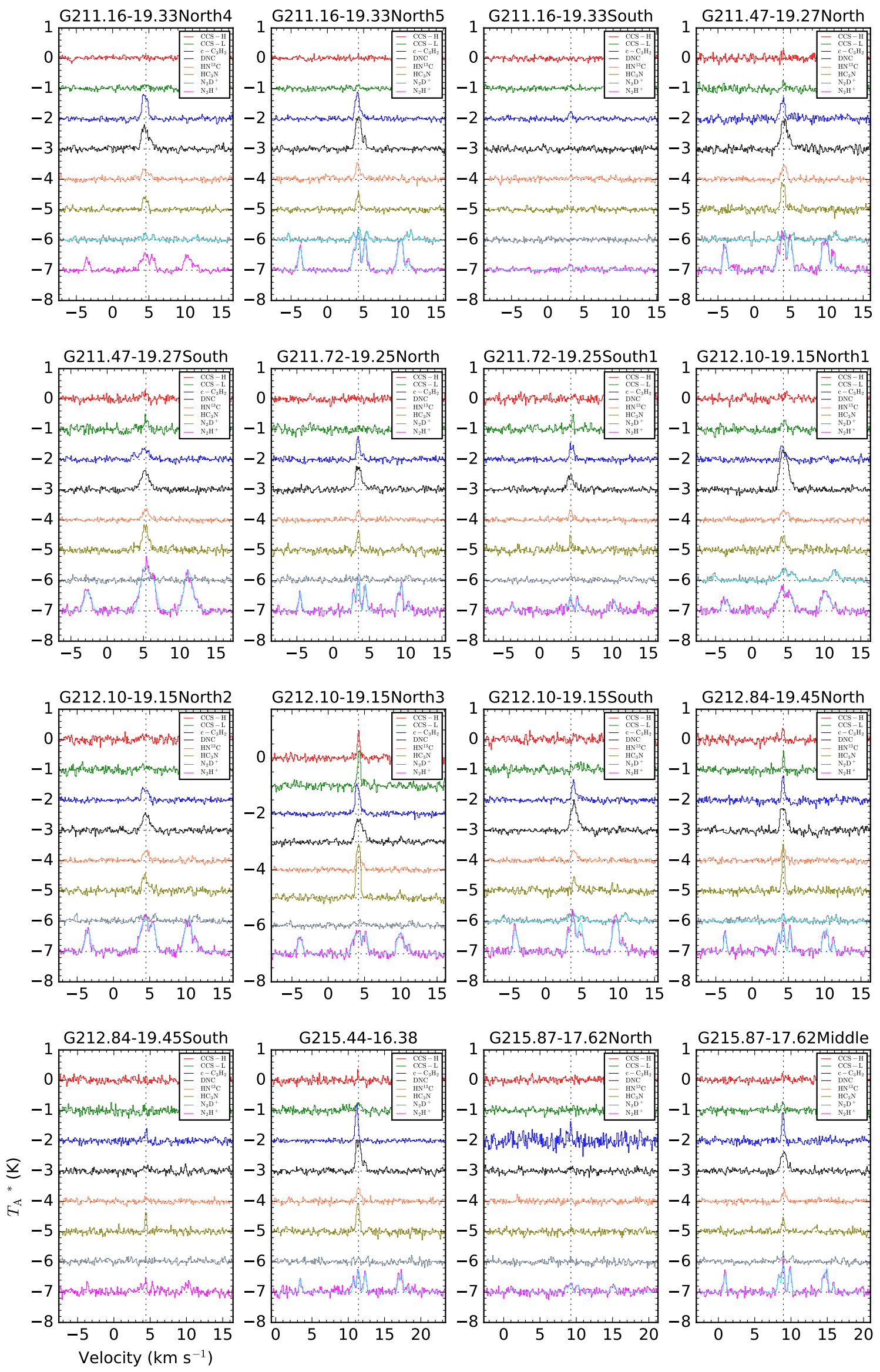

Figure 3. (continued) 

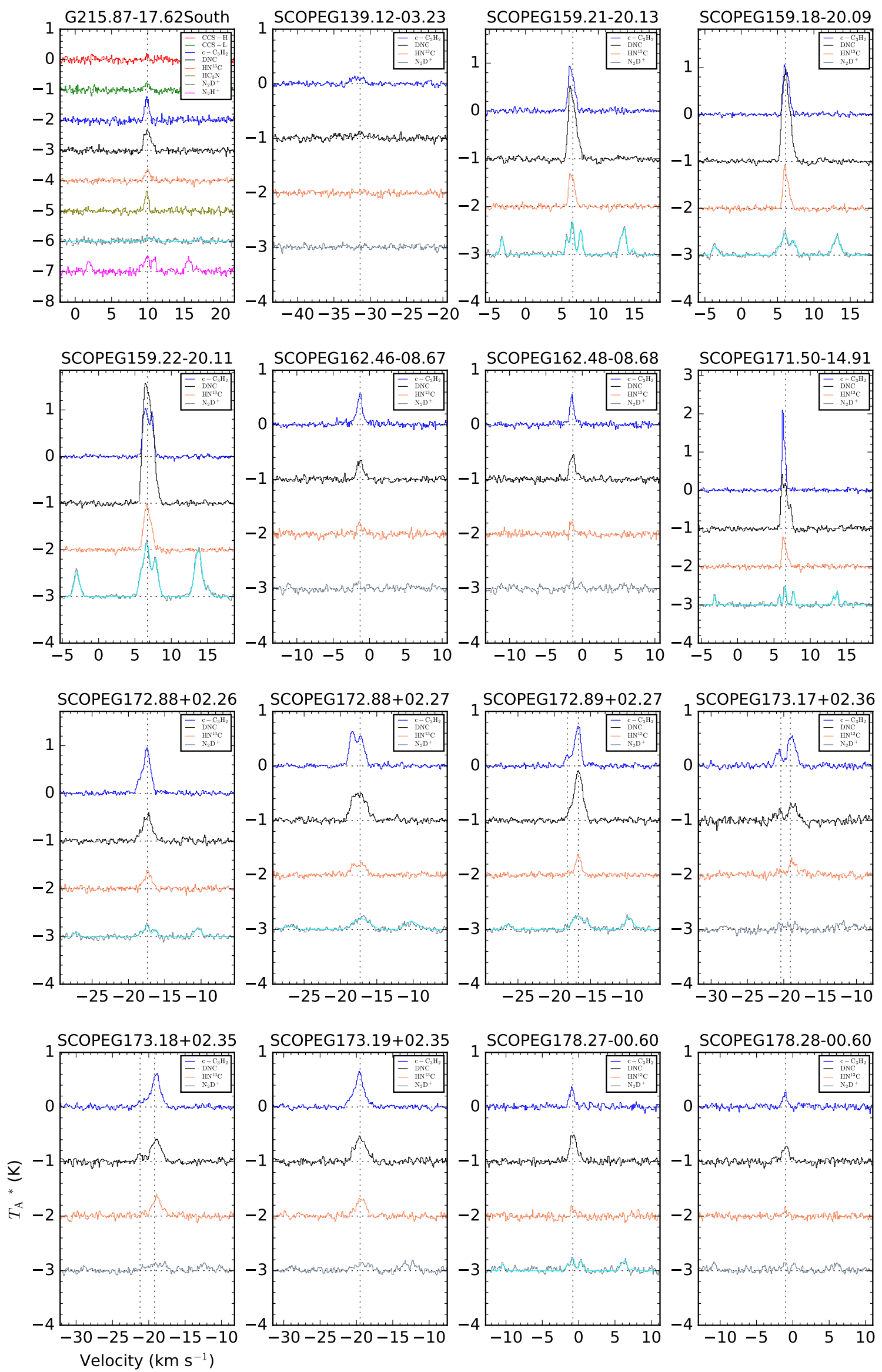

Figure 3. (continued) 

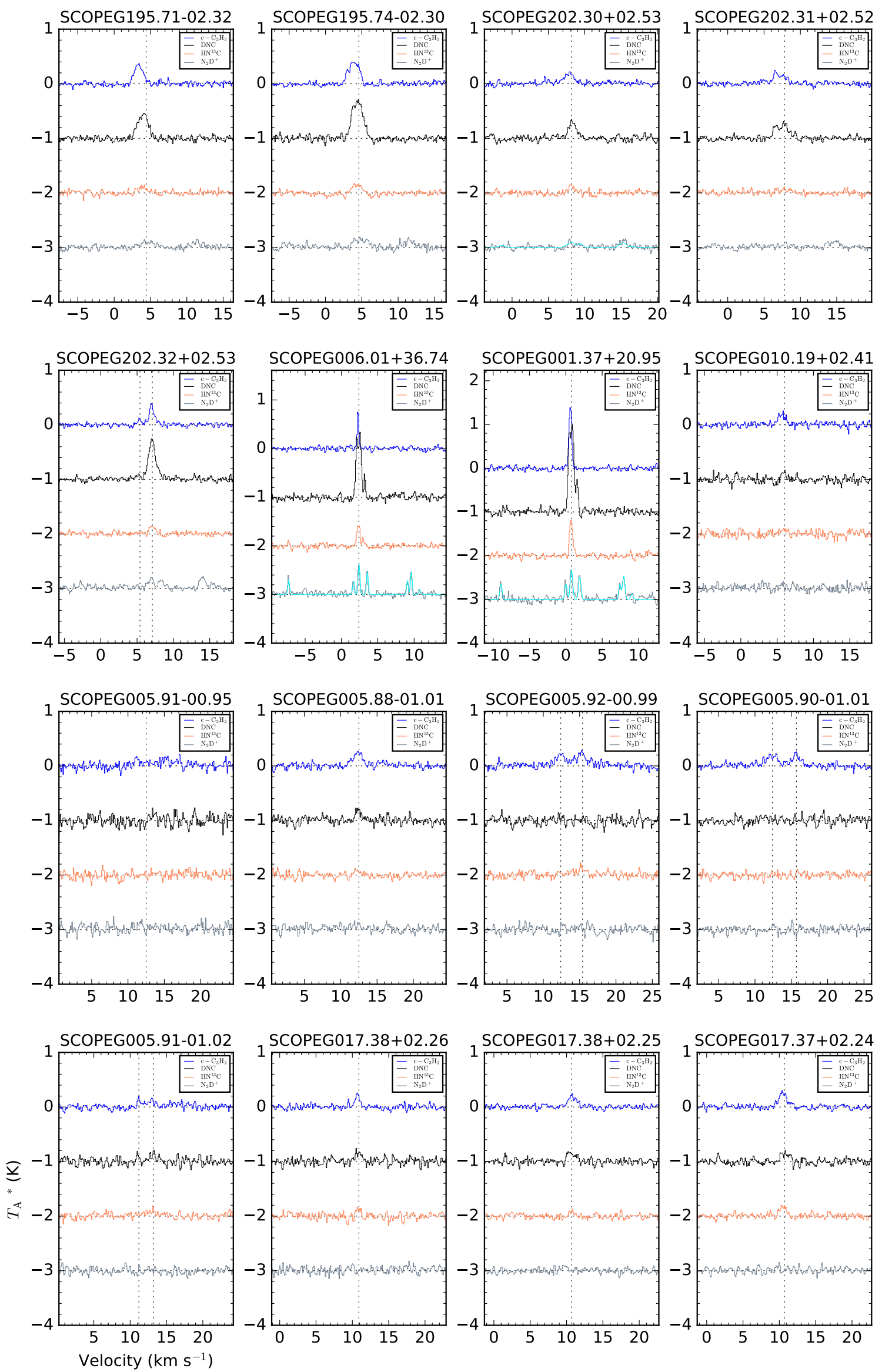

Figure 3. (continued) 

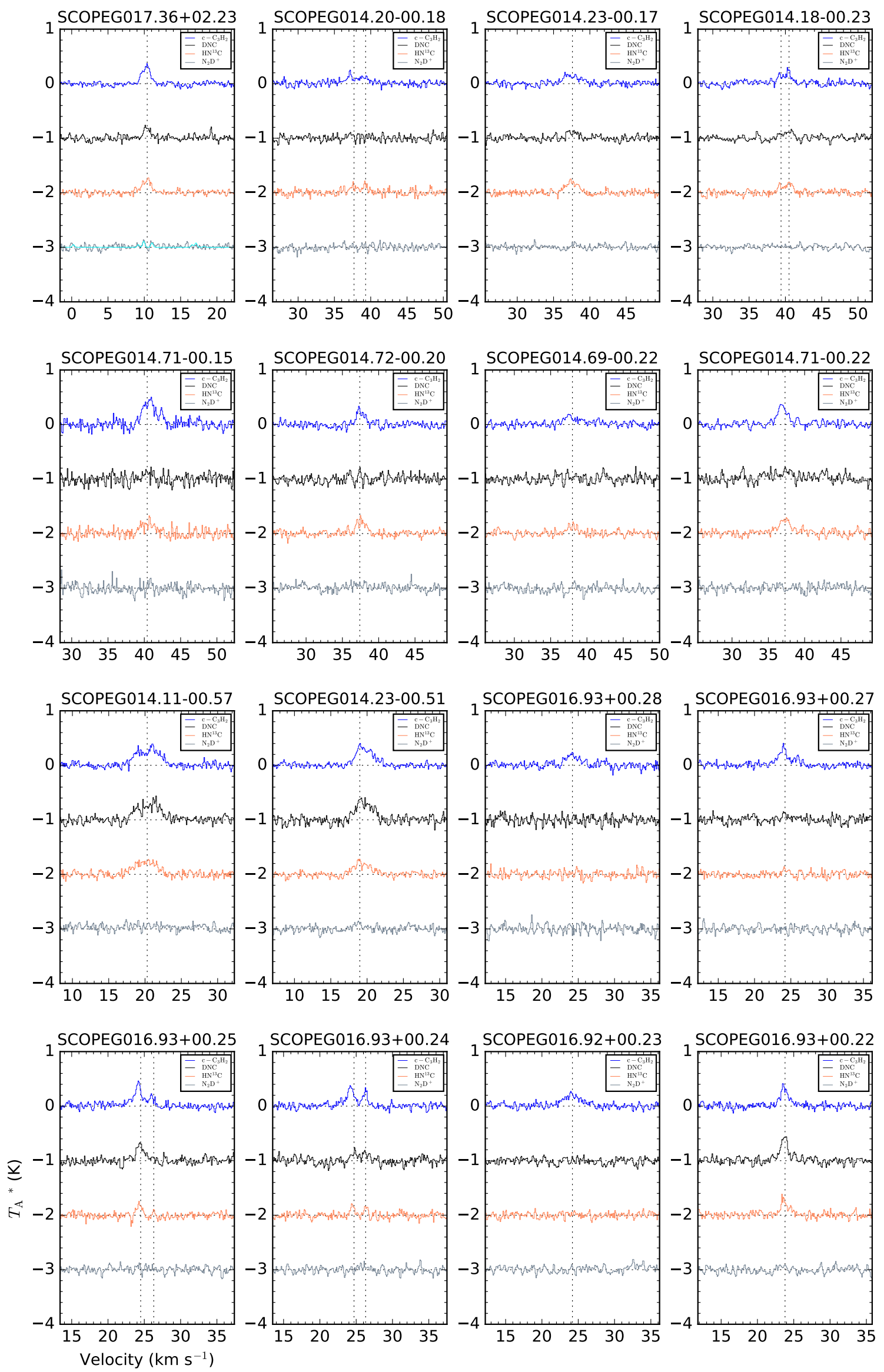

Figure 3. (continued) 

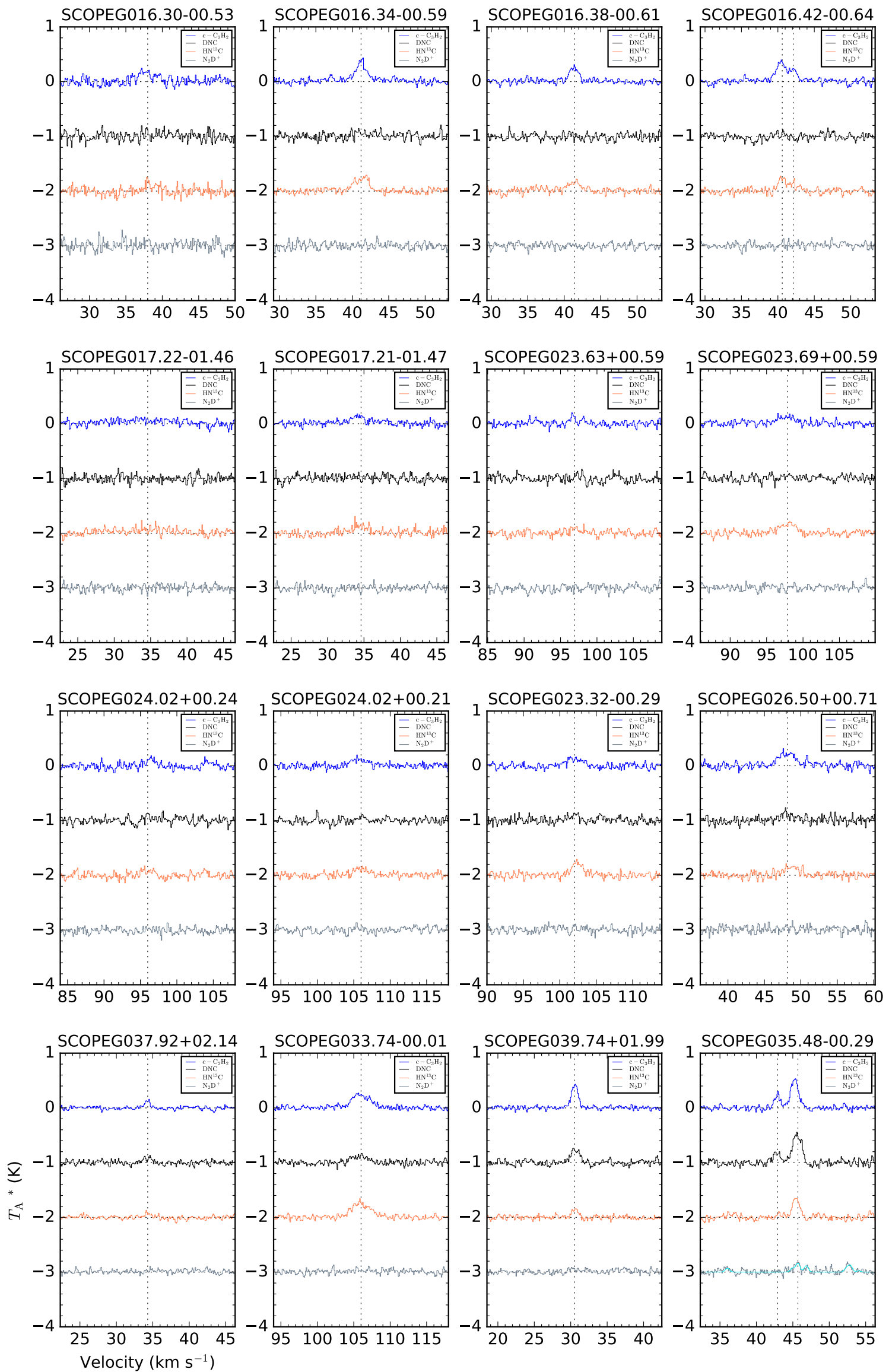

Figure 3. (continued) 

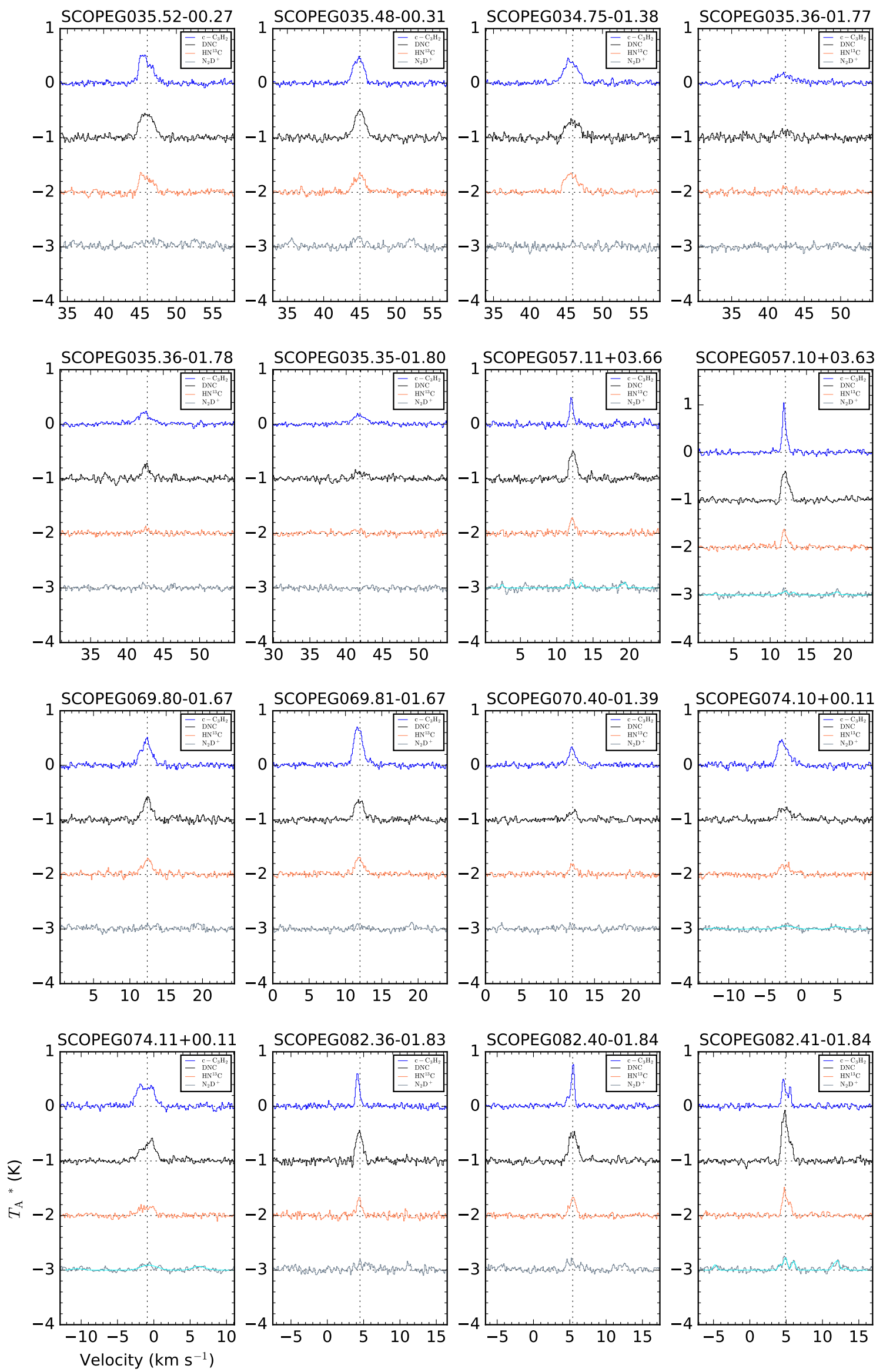

Figure 3. (continued) 

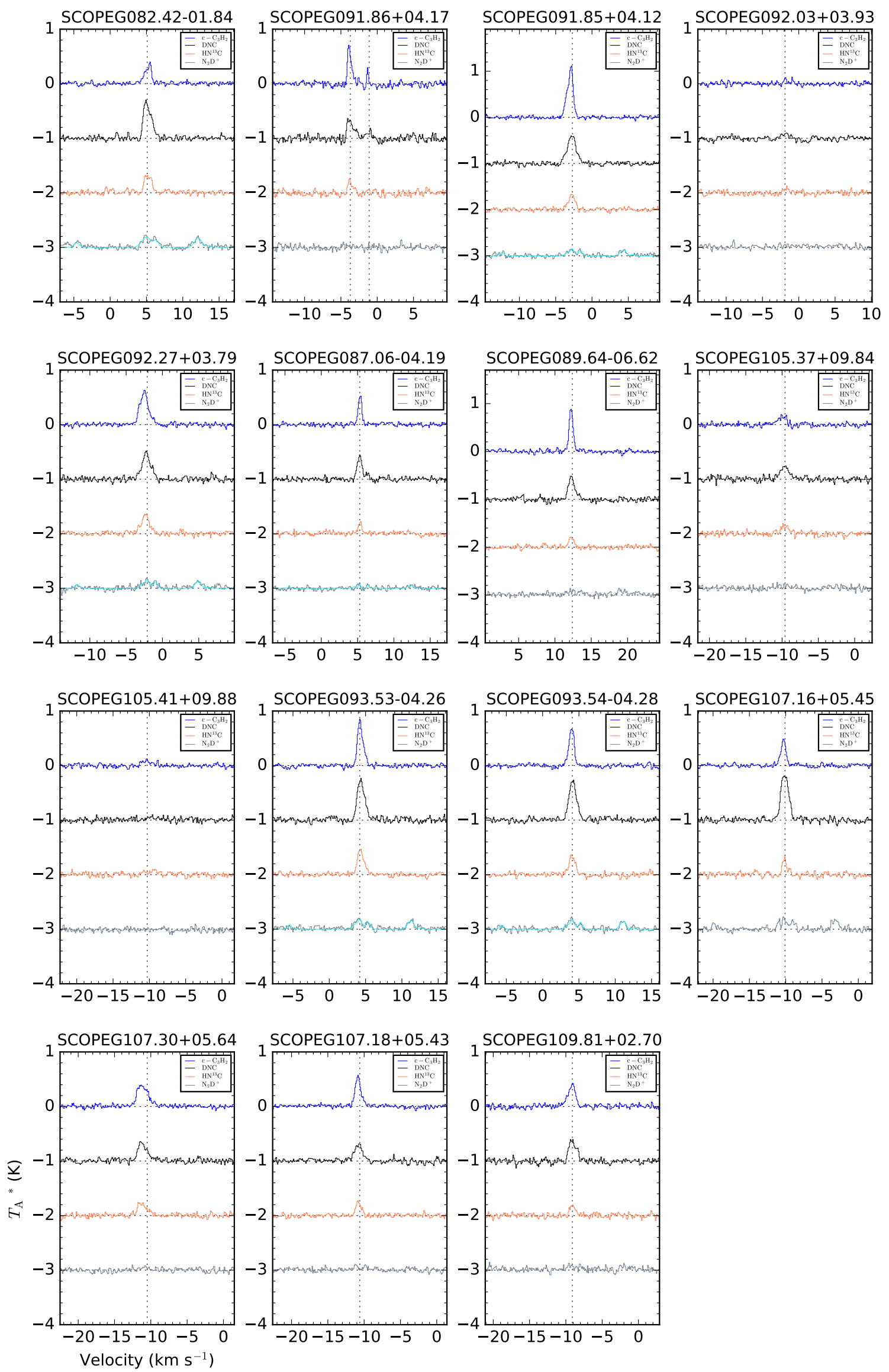

Figure 3. (continued) 


\section{All Authors and Affiliations}

Gwanjeong Kim, ${ }^{1}$ Ken'ichi Tatematsu, ${ }^{1,2}$ Tie Liu, ${ }^{3}$ Hee-weon Yi, ${ }^{4}$ Jinhua He, ${ }^{5,6,7}$ NaOmi Hirano, ${ }^{8}$ Sheng-Yuan Liu ${ }^{8}$ Minho Choi, ${ }^{9}$ Patricio Sanhueza, ${ }^{10}$ L. Viktor Tóth, ${ }^{11}$ Neal J. Evans II, ${ }^{12}$ Siyi Feng, ${ }^{13,10,14}$ Mika Juvela, ${ }^{15}$ Kee-Tae Kim, ${ }^{9}$ Charlotte VAStel, ${ }^{16}$ Jeong-Eun Lee, ${ }^{4}$ Quang NguyễN Lu'O'NG, ${ }^{10,9,17}$ Miju Kang, ${ }^{9}$ Isabelle Ristorcelli, ${ }^{18}$ O. Fehér, ${ }^{19}$ Yuefang Wu,${ }^{20}$ Satoshi Ohashi, ${ }^{21}$ Ke Wang, ${ }^{22}$ Ryo Kandori, ${ }^{10}$ Tomoya Hirota, ${ }^{10,2}$ Takeshi Sakai, ${ }^{23}$ Xing Lu,${ }^{10}$ Mark A. Thompson, ${ }^{24}$ Gary A. Fuller, ${ }^{25}$ Di Li, ${ }^{26}$ Hiroko Shinnaga, ${ }^{27}$ And Jungha Kim ${ }^{10,2}$

${ }^{1}$ Nobeyama Radio Observatory, National Astronomical Observatory of Japan, National Institutes of Natural Sciences, Nobeyama, Minamimaki, Minamisaku, Nagano 384-1305, Japan

${ }^{2}$ Department of Astronomical Science, SOKENDAI (The Graduate University for Advanced Studies), 2-21-1 Osawa, Mitaka, Tokyo 181-8588, Japan

${ }^{3}$ Shanghai Astronomical Observatory, Chinese Academy of Sciences, 80 Nandan Road, Shanghai 200030, Peoples Republic of China

${ }^{4}$ School of Space Research, Kyung Hee University, Seocheon-Dong, Giheung-Gu, Yongin-Si, Gyeonggi-Do, 446-701, South Korea

${ }^{5}$ Yunnan Observatories, Chinese Academy of Sciences, 396 Yangfangwang, Guandu District, Kunming, 650216, P. R. China

${ }^{6}$ Chinese Academy of Sciences South America Center for Astronomy, National Astronomical Observatories, CAS, Beijing 100101, China ${ }^{7}$ Departamento de Astronomía, Universidad de Chile, Casilla 36-D, Santiago, Chile

${ }^{8}$ Academia Sinica Institute of Astronomy and Astrophysics, $11 \mathrm{~F}$ of Astronomy-Mathematics Building, AS/NTU. No.1, Sec. 4, Roosevelt Rd, Taipei 10617, Taiwan, R.O.C.

${ }^{9}$ Korea Astronomy and Space Science Institute, Daedeokdaero 776, Yuseong, Daejeon 305-348, South Korea

${ }^{10}$ National Astronomical Observatory of Japan, National Institutes of Natural Sciences, 2-21-1 Osawa, Mitaka, Tokyo 181-8588, Japan

${ }^{11}$ Department of Astronomy, Eötvös Loránd Unviersity, Pázmany Péter sétny 1, 1117 Budapest, Hungary

${ }^{12}$ Department of Astronomy, The University of Texas at Austin, 2515 Speedway, Stop C1400, Austin, TX 78712-1205, USA

${ }^{13}$ Chinese Academy of Sciences Key Laboratory of FAST, National Astronomical Observatory of China, Datun Road 20, Chaoyang, Beijing, 100012, P. R. China

${ }^{14}$ Academia Sinica Institute of Astronomy and Astrophysics, No.1, Sec. 4, Roosevelt Rd, Taipei 10617, Taiwan, Republic of China ${ }^{15}$ Department of Physics, P.O.Box 64, FI-00014, University of Helsinki, Finland

${ }^{16}$ IRAP, Universit de Toulouse, CNRS, CNES, UPS, (Toulouse), France

${ }^{17}$ IBM, Canada

${ }^{18}$ RAP, CNRS (UMR5277), Universite Paul Sabatier, 9 avenue du Colonel Roche, BP 44346, 31028, Toulouse Cedex 4, France

${ }^{19}$ Institut de Radioastronomie Millimetrique, 300 Rue de la Piscine, 38406, Saint Martin d'Heres, France

${ }^{20}$ Department of Astronomy, Peking University, 100871, Beijing, China

${ }^{21}$ The Institute of Physical and Chemical Research (RIKEN), 2-1, Hirosawa, Wako-shi, Saitama 351-0198, Japan

${ }^{22}$ Kavli Institute for Astronomy and Astrophysics, Peking University, 5 Yiheyuan Road, Haidian District, Beijing 100871, China

${ }^{23}$ Graduate School of Informatics and Engineering, The University of Electro-Communications, Chofu, Tokyo 182-8585, Japan

${ }^{24}$ Centre for Astrophysics Research, Science 85 Technology Research Institute, University of Hertfordshire, Hatfield, AL10 9AB, UK

${ }^{25}$ Jodrell Bank Centre for Astrophysics, School of Physics and Astronomy, University of Manchester, Oxford Road, Manchester, M13 $9 P L, U K$

${ }^{26}$ National Astronomical Observatories, Chinese Academy of Sciences, Beijing, 100012, China

${ }^{27}$ Department of Physics, Kagoshima University, 1-21-35, Korimoto, Kagoshima, 890-0065, Japan 